\title{
Malnutrition in Hospitalized Adults: A Systematic Review
}




\section{Malnutrition in Hospitalized Adults: A Systematic Review}

Prepared for:

Agency for Healthcare Research and Quality

U.S. Department of Health and Human Services

5600 Fishers Lane

Rockville, MD 20857

www.ahrq.gov

Contract No. 75Q80120D00002

Prepared by:

ECRI-Penn Medicine Evidence-based Practice Center

Plymouth Meeting, PA

Investigators:

Stacey Uhl, M.S.

Shazia Mehmood Siddique, M.D., M.P.H.

Liam McKeever, Ph.D., R.D.N.

Aaron Bloschichak, M.P.H.

Kristen D'Anci, Ph.D.

Brian Leas, M.S., M.A.

Nikhil K. Mull, M.D.

Amy Y. Tsou, M.D., M.Sc.

AHRQ Publication No. 21(22)-EHC035

October 2021 
This report is based on research conducted by the ECRI-Penn Medicine Evidence-based Practice Center under contract to the Agency for Healthcare Research and Quality (AHRQ), Rockville, MD (Contract No. 75Q80120D00002). The findings and conclusions in this document are those of the authors, who are responsible for its contents; the findings and conclusions do not necessarily represent the views of AHRQ. Therefore, no statement in this report should be construed as an official position of AHRQ or of the U.S. Department of Health and Human Services.

\section{None of the investigators have any affiliations or financial involvement that conflicts with the material presented in this report.}

The information in this report is intended to help healthcare decision makers - patients and clinicians, health system leaders, and policymakers, among others-make well-informed decisions and thereby improve the quality of healthcare services. This report is not intended to be a substitute for the application of clinical judgment. Anyone who makes decisions concerning the provision of clinical care should consider this report in the same way as any medical reference and in conjunction with all other pertinent information, i.e., in the context of available resources and circumstances presented by individual patients.

This report is made available to the public under the terms of a licensing agreement between the author and the Agency for Healthcare Research and Quality. This report may be used and reprinted without permission except those copyrighted materials that are clearly noted in the report. Further reproduction of those copyrighted materials is prohibited without the express permission of copyright holders.

AHRQ or U.S. Department of Health and Human Services endorsement of any derivative products that may be developed from this report, such as clinical practice guidelines, other quality enhancement tools, or reimbursement or coverage policies, may not be stated or implied.

AHRQ appreciates appropriate acknowledgment and citation of its work. Suggested language for acknowledgment: This work was based on an evidence report, Malnutrition in Hospitalized Adults: A Systematic Review, by the ECRI-Penn Medicine Evidence-based Practice Center Program at the Agency for Healthcare Research and Quality (AHRQ).

Suggested citation: Uhl S, Siddique SM, McKeever L, Bloschichak A, D’Anci K, Leas B, Mull NK, Tsou AY. Malnutrition in Hospitalized Adults: A Systematic Review. Comparative Effectiveness Review No. 249. (Prepared by the ECRI-Penn Medicine Evidence-based Practice Center under Contract No. 75Q80120D00002.) AHRQ Publication No. 21(22)-EHC035. Rockville, MD: Agency for Healthcare Research and Quality; October 2021. DOI: https://doi.org/10.23970/AHRQEPCCER249. Posted final reports are located on the Effective Health Care Program search page. 


\section{Preface}

The Agency for Healthcare Research and Quality (AHRQ), through its Evidence-based Practice Centers (EPCs), sponsors the development of evidence reports and technology assessments to assist public- and private-sector organizations in their efforts to improve the quality of healthcare in the United States.

In fiscal year 2020, Congress requested that AHRQ convene a panel of experts charged with developing quality measures for malnutrition-related hospital readmissions. These measures would be intended to support assigning accountability for the assessment and treatment of malnutrition in hospitalized adults, with an emphasis on the needs of older frail adults. This systematic review is intended to support the efforts of the panel.

The reports and assessments provide organizations with comprehensive, evidence-based information on common medical conditions and new healthcare technologies and strategies. They also identify research gaps in the selected scientific area, identify methodologic and scientific weaknesses, suggest research needs, and move the field forward through an unbiased, evidence-based assessment of the available literature. The EPCs systematically review the relevant scientific literature on topics assigned to them by AHRQ and conduct additional analyses when appropriate prior to developing their reports and assessments.

To bring the broadest range of experts into the development of evidence reports and health technology assessments, AHRQ encourages the EPCs to form partnerships and enter into collaborations with other medical and research organizations. The EPCs work with these partner organizations to ensure that the evidence reports and technology assessments they produce will become building blocks for healthcare quality improvement projects throughout the Nation. The reports undergo peer review and public comment prior to their release as a final report.

AHRQ expects that the EPC evidence reports and technology assessments, when appropriate, will inform individual health plans, providers, and purchasers as well as the healthcare system as a whole by providing important information to help improve healthcare quality.

If you have comments on this evidence report, they may be sent by mail to the Task Order Officer named below at: Agency for Healthcare Research and Quality, 5600 Fishers Lane, Rockville, MD 20857, or by email to epc@ahrq.hhs.gov.

David Meyers, M.D.

Acting Director

Agency for Healthcare Research and Quality

Craig Umscheid, M.D., M.S.

Director, Evidence-based Practice Center

Program

Center for Evidence and Practice

Improvement

Agency for Healthcare Research and Quality
Arlene S. Bierman, M.D., M.S.

Director

Center for Evidence and Practice

Improvement

Agency for Healthcare Research and Quality

Jill Huppert, M.D., M.P.H.

Task Order Officer

Center for Evidence and Practice

Improvement

Agency for Healthcare Research and Quality 


\section{Acknowledgments}

The authors gratefully acknowledge the following individuals at AHRQ for their guidance: Marian S. McDonagh, Pharm.D., who served as Associate Editor; Jill Huppert, M.D., M.P.H., who served as Task Order Officer; Pamela L. Owens, Ph.D., and Mamatha Pancholi, M.S., quality measurement experts who served as liaisons to AHRQ's Technical Expert Panel on Quality Measurement of Malnutrition in Hospitalized Patients; and Christine Chang, M.D., M.P.H., who served as Acting Director. We are grateful to the following subject matter experts for sharing their depth of knowledge and expertise in this topic to inform all stages of this report: Charlene Compher, Ph.D., R.D.; James D. Lewis, M.D., M.S.C.E.; and Gary D. Wu, M.D. We also thank the following individuals at ECRI for their contributions to this project: Katherine Donahue, Jennifer Maslin, and Rebecca Rishar, M.S.L.I.S.

\section{Technical Expert Panel}

In designing the study questions and methodology at the outset of this report, the EPC consulted several technical and content experts. Broad expertise and perspectives were sought. Divergent and conflicted opinions are common and perceived as healthy scientific discourse that results in a thoughtful, relevant systematic review. Therefore, in the end, study questions, design, methodologic approaches, and/or conclusions do not necessarily represent the views of individual technical and content experts.

Technical Experts must disclose any financial conflicts of interest greater than $\$ 5,000$ and any other relevant business or professional conflicts of interest. Because of their unique clinical or content expertise, individuals with potential conflicts may be retained. The TOO and the EPC work to balance, manage, or mitigate any potential conflicts of interest identified.

The list of Technical Experts who provided input to this report follows:

Peggi Guenter, Ph.D., R.N., FAAN, FASPEN*

Senior Director for Clinical Practice, Quality, and Advocacy

American Society for Parenteral and Enteral Nutrition (ASPEN)

Silver Spring, MD

Lauren Hudson, M.S., R.D., L.D.N.*

Assistant Executive Hospital Director and Director of Clinical Nutrition

University of Pennsylvania, Hospital of the University of Pennsylvania

Philadelphia, PA

Jeremy J. Michel, M.D., M.H.S.*

Pediatrician and Clinical Informaticist

Children's Hospital of Philadelphia Research Institute

Philadelphia, PA

Patricia Sheean, Ph.D., R.D.N.*

Associate Professor

Parkinson School of Health Sciences and Public Health

Loyola University Chicago

Chicago, IL 
Alison Steiber, Ph.D., R.D.N.*

Chief Science Officer

Academy of Nutrition and Dietetics

Chicago, IL

*Provided input on Draft Report

\section{Peer Reviewers}

Before publication of the final evidence report, the EPCs sought input from independent peer reviewers without financial conflicts of interest. However, the conclusions and synthesis of the scientific literature presented in this report do not necessarily represent the views of individual reviewers.

Peer reviewers must disclose any financial conflicts of interest greater than $\$ 5,000$ and any other relevant business or professional conflicts of interest. Because of their unique clinical or content expertise, individuals with potential nonfinancial conflicts may be retained. The TOO and the EPC work to balance, manage, or mitigate any potential nonfinancial conflicts of interest identified.

The list of Peer Reviewers follows:

JohnMarc Alban, M.S., B.S.

Associate Director for Quality Measurement and Informatics

The Joint Commission

Oak Brook, IL

Tommy Cederholm, M.D., Ph.D.

Professor of Medicine and Clinical Nutrition

Uppsala University

Uppsala, Sweden

Christopher Lynch, Ph.D.

Acting Director of the Office of Nutrition Research

National Institutes of Health

Bethesda, MD

Marion F. Winkler, Ph.D., R.D., L.D.N, C.N.S.C.

Surgical Nutrition Specialist and Professor of Surgery

Rhode Island Hospital, Brown Medical School

Providence, RI 


\section{Malnutrition in Hospitalized Adults: A Systematic Review}

\section{Structured Abstract}

Objectives. To review the association between malnutrition and clinical outcomes among hospitalized patients, evaluate effectiveness of measurement tools for malnutrition on clinical outcomes, and assess effectiveness of hospital-initiated interventions for patients diagnosed with malnutrition.

Data sources. We searched electronic databases $\left(\right.$ Embase $^{\circledR}, \operatorname{MEDLINE}^{\circledR}, \operatorname{PubMed}^{\circledR}$, and the Cochrane Library) from January 1, 2000, to June 3, 2021. We hand-searched reference lists of relevant studies and searched for unpublished studies in ClinicalTrials.gov.

Review methods. Using predefined criteria and dual review, we selected (1) existing systematic reviews (SRs) to assess the association between malnutrition and clinical outcomes,

(2) randomized and non-randomized studies to evaluate the effectiveness of malnutrition tools on clinical outcomes, and (3) randomized controlled trials (RCTs) to assess effectiveness of hospital-initiated treatments for malnutrition. Clinical outcomes of interest included mortality, length of stay, 30-day readmission, quality of life, functional status, activities of daily living, hospital acquired conditions, wound healing, and discharge disposition. When appropriate, we conducted meta-analysis to quantitatively summarize study findings; otherwise, data were narratively synthesized. When available, we used pooled estimates from existing SRs to determine the association between malnutrition and clinical outcomes, and assessed the strength of evidence.

Results. Six existing SRs (including 43 unique studies) provided evidence on the association between malnutrition and clinical outcomes. Low to moderate strength of evidence (SOE) showed an association between malnutrition and increased hospital mortality and prolonged hospital length of stay. This association was observed across patients hospitalized for an acute medical event requiring intensive care unit care, heart failure, and cirrhosis. Literature searches found no studies that met inclusion criteria and assessed effectiveness of measurement tools. The primary reason studies did not meet inclusion criteria is because they lacked an appropriate control group. Moderate SOE from 11 RCTs found that hospital-initiated malnutrition interventions likely reduce mortality compared with usual care among hospitalized patients diagnosed with malnutrition. Low SOE indicated that hospital-initiated malnutrition interventions may also improve quality of life compared to usual care.

Conclusions. Evidence shows an association between malnutrition and increased mortality and prolonged length of hospital stay among hospitalized patients identified as malnourished.

However, the strength of this association varied depending on patient population and tool used to identify malnutrition. Evidence indicates malnutrition-focused hospital-initiated interventions likely reduce mortality and may improve quality of life compared to usual care among patients diagnosed with malnutrition. Research is needed to assess the clinical utility of measurement tools for malnutrition. 


\section{Contents}

Evidence Summary ........................................................................................................................... ES-1

Introduction ................................................................................................................................ 1

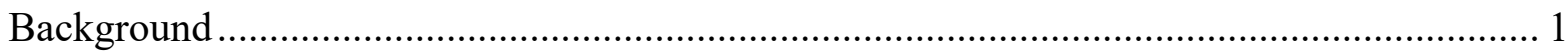

Purpose and Scope of the Systematic Review ………........................................................ 4

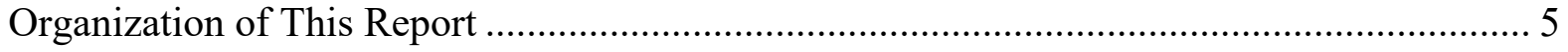

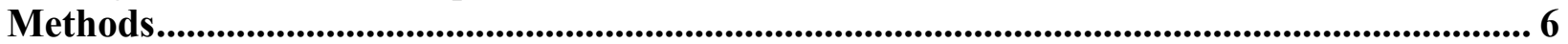

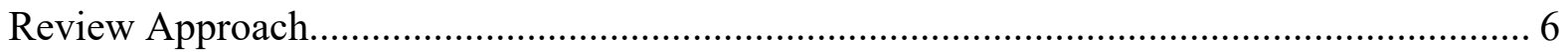

Role of the Technical Expert Panel ............................................................................. 6

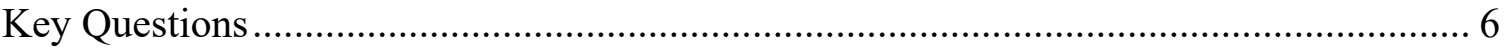

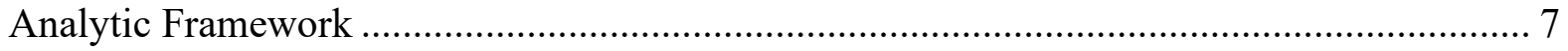

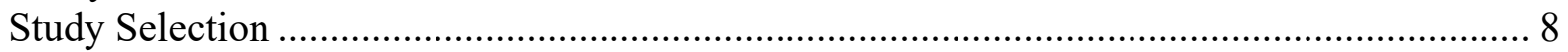

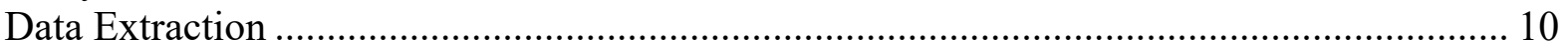

Risk of Bias Assessment......................................................................................... 11

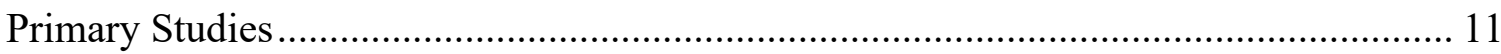

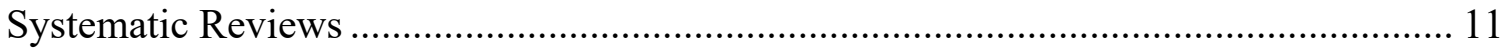

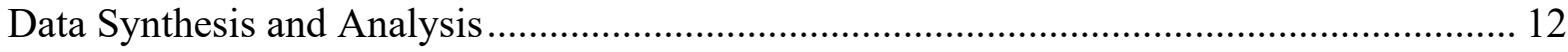

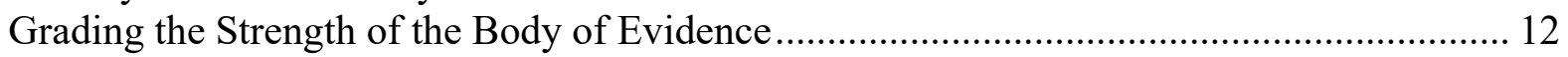

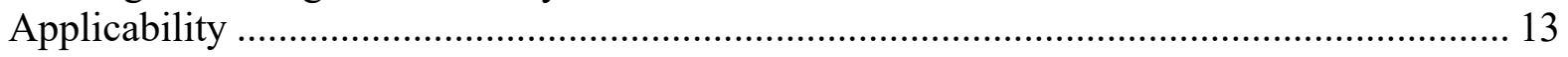

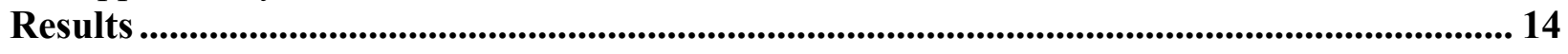

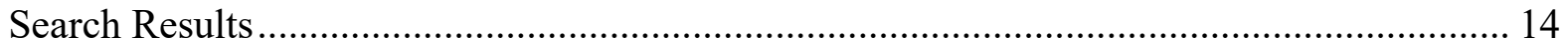

Key Question 1. What is the association between malnutrition and clinical outcomes among hospitalized patients? ................................................................................................... 16

a. How do outcomes vary depending on measures or tools used to detect malnutrition? 16

b. Are patient-related risk factors, such as increased age or certain pre-existing health conditions, associated with poorer clinical outcomes? ....................................................... 16

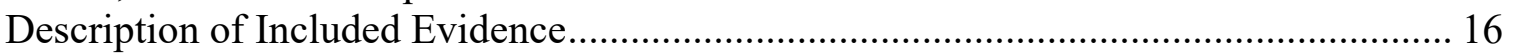

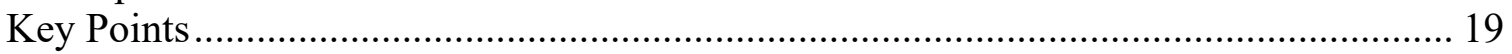

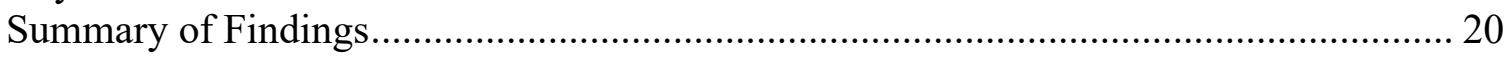

Key Question 2. What is the effectiveness of screening or diagnostic assessment for

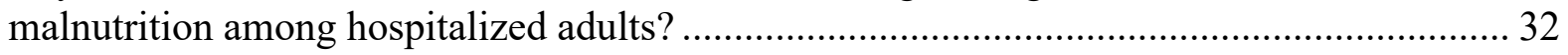

a. In studies that report on clinical outcomes, what is the accuracy of screening or diagnostic tools for malnutrition? ………………............................................................ 32

b. In studies that report on clinical outcomes, what is the effectiveness of screening or diagnostic tools on measures of nutrition (nutritional stores)? ............................................... 32

c. What is the impact of the use of screening or diagnostic tools on clinical outcomes?.. 32

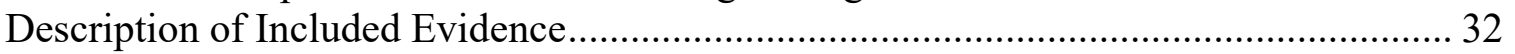

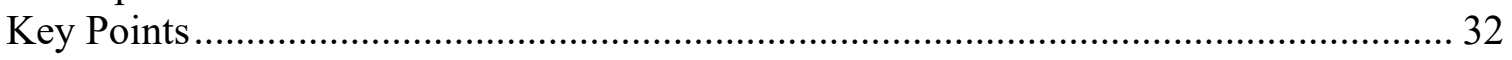

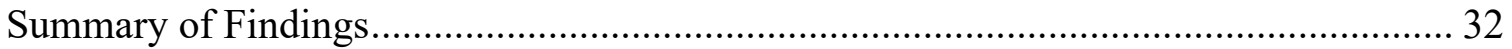

Key Question 3. Among patients diagnosed with malnutrition, what is the effectiveness of hospital-initiated interventions used to treat malnutrition on clinical outcomes? ................... 37

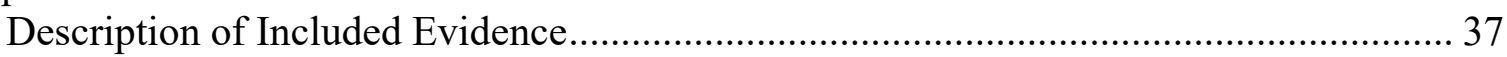

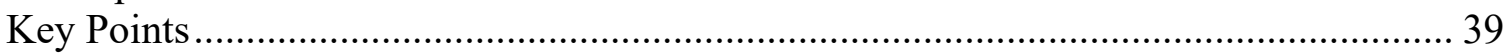

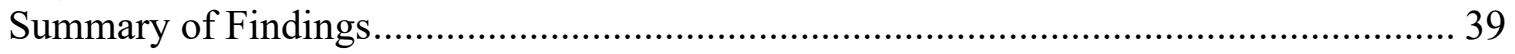

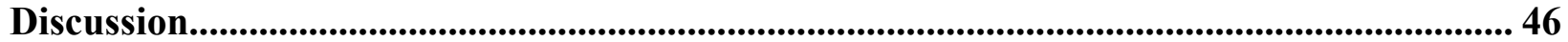

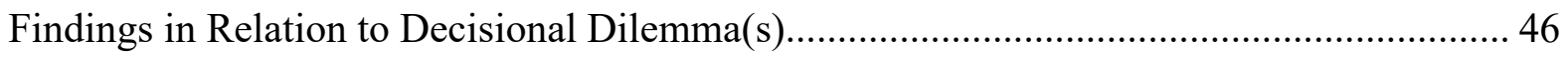




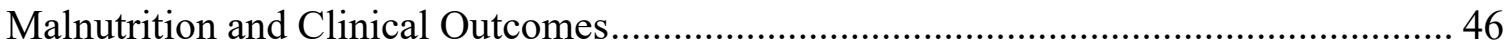

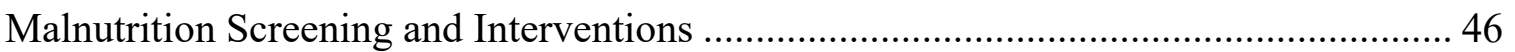

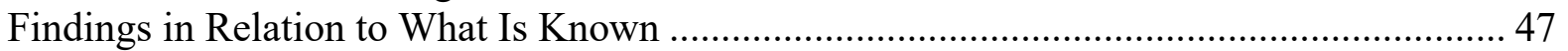

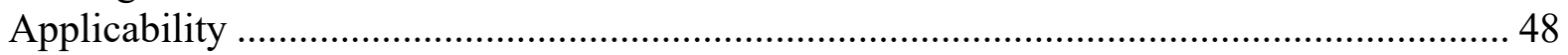

Limitations and Suggestions for Future Research ……….................................................. 48

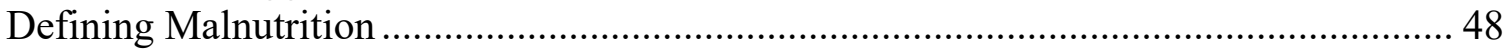

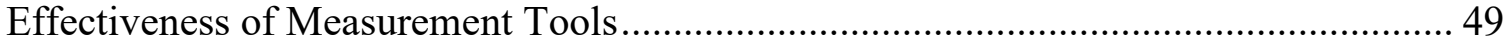

Malnutrition Interventions ..................................................................................... 49

Implications for Clinical Practice, Education, Research, or Health Policy ............................ 50

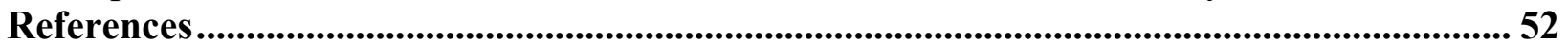

\section{Tables}

Table 1. Commonly available measurement tools and criteria for assessing nutritional status ..... 2

Table 2. PICOTS (population, intervention, comparator, outcome, timing, setting) ..................... 9

Table 3. Key characteristics of included systematic reviews ..................................................... 17

Table 4. Strength of evidence ratings by measurement tool for trauma injury/ICU patients ........ 22

Table 5. Strength of evidence ratings by measurement tool for patients with chronic diseases... 29

Table 6. Evaluation of PICOs of studies excluded at full-text level for Key Question 2 2............ 35

Table 7. Characteristics of studies assessing the effect of hospital-initiated intervention to

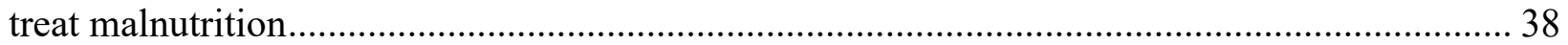

Table 8. Strength of evidence ratings for interventions for malnutrition in patients diagnosed

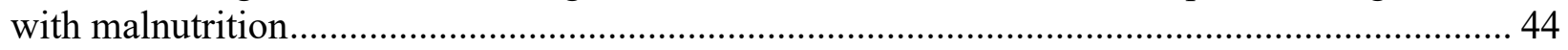

\section{Figures}

Figure 1. Analytic framework for malnutrition in hospitalized adults ........................................ 7

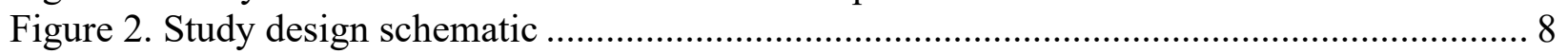

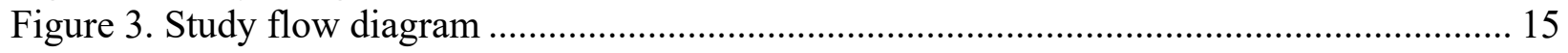

Figure 4. Effect of hospital-initiated interventions on mortality, all studies ................................ 40

Figure 5. Effect of hospital-initiated interventions on quality of life, subgroup quality of life

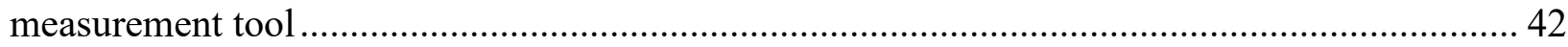

\section{Appendixes}

Appendix A. Search Strategy ………………………….................................................. A-1

Appendix B. Methods ………………………………………………………………... B-1

Appendix C. Excluded Studies …………………………………………………………... C-1

Appendix D. Characteristics of Included Studies ................................................................ D-1

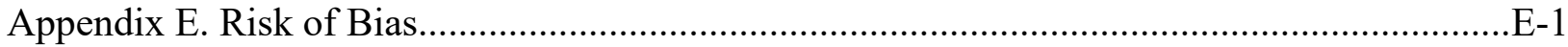

Appendix F. Results From Included Studies ………………............................................... F-1

Appendix G. Forest Plots of Additional Analysis ………………………………………...... G-1

Appendix H. Additional Information for KQ 1 .............................................................. H-1

Appendix I. Appendix References.......................................................................................... 


\section{Evidence Summary}

\section{Main Points}

\section{Association Between Malnutrition and Clinical Outcomes}

- Patients requiring intensive care unit (ICU) care and diagnosed with malnutrition (using Subjective Global Assessment [SGA]) may have higher hospital mortality compared to well-nourished patients requiring ICU care.

- Patients requiring ICU care and diagnosed with malnutrition (using SGA) are likely to experience prolonged hospital length of stay compared to well-nourished patients requiring ICU care.

- Patients requiring ICU care and diagnosed with malnutrition (using Mini Nutritional Assessment [MNA]) may experience more hospital acquired complications compared to well-nourished patients requiring ICU care.

- Patients hospitalized due to traumatic injury and screened at risk of malnutrition (using Nutritional Risk Screening [NRS]-2002) may experience more hospital acquired conditions compared to well-nourished patients.

- Patients hospitalized with heart failure and diagnosed with malnutrition (using several different measurement tools) may have higher mortality compared to well-nourished patients with heart failure.

- Patients hospitalized with cancer and diagnosed with malnutrition (using SGA) may experience prolonged hospital length of stay compared to well-nourished patients.

- Patients hospitalized with cirrhosis awaiting transplantation and diagnosed with malnutrition (using SGA) may have higher pre-transplant mortality compared to wellnourished patients.

\section{Effectiveness of Screening on Clinical Outcomes}

- No studies met inclusion criteria to address effectiveness of screening or diagnostic assessment on clinical outcomes, primarily because studies lacked an appropriate control group.

- This evidence gap underscores the need for future research that addresses the effectiveness of various measurement tools for malnutrition on clinical outcomes. Such research is vital to standardize malnutrition assessment and further understand its downstream implications on patient-relevant outcomes.

\section{Effectiveness of Hospital-Initiated Interventions for Malnutrition}

- Hospital-initiated malnutrition interventions (i.e., specialized nutrition care, protein/calorie supplementation) likely decrease mortality compared to usual care.

- Hospital-initiated malnutrition interventions may improve quality of life compared to usual care. 
- No difference was observed between hospital-initiated malnutrition interventions and usual care for length of stay, readmission rates, and hospital acquired conditions compared to usual care.

- Evidence was insufficient to address the effect of hospital-initiated malnutrition interventions on activities of daily living and discharge disposition compared to usual care.

\section{Background and Purpose}

In fiscal year 2020, Congress requested that the Agency for Healthcare Research and Quality (AHRQ) convene a panel of experts charged with developing quality measures for malnutritionrelated hospital readmissions. At AHRQ's request, we conducted a systematic review to inform the potential development of these measures. Our Key Questions addressed the following: (1) reviewing the association between malnutrition and clinical outcomes, (2) evaluating the effectiveness of measurement tools of malnutrition on clinical outcomes, and (3) assessing the effectiveness of hospital-initiated interventions to treat patients diagnosed with malnutrition. Understanding downstream consequences of malnutrition screening is extremely important as US hospitals are mandated to provide nutrition screening for all hospitalized patients within 24 hours of admission.

\section{Methods}

Electronic databases (MEDLINE ${ }^{\circledR}$, Embase $^{\circledR}$, and Cochrane Library) were searched from January 1, 2000, to June 3, 2021. We hand-searched the reference lists of relevant studies and searched for unpublished studies in ClinicalTrials.gov.

Using predefined criteria, we selected (1) existing systematic reviews (SRs) assessing the association between malnutrition and clinical outcomes, (2) randomized and non-randomized studies evaluating the effectiveness of screening or diagnostic assessment on clinical outcomes, and (3) randomized controlled trials (RCTs) assessing hospital-initiated treatments for malnutrition. We only included studies of hospitalized patients aged 18 years or older.

Malnutrition was defined based on commonly available diagnostic assessment tools, such as SGA, or MNA. Interventions of interest included measurement tools and treatments initiated within the hospital and intended to impact nutritional status. Clinical outcomes of interest included mortality, length of stay, 30-day readmission, quality of life, functional status, activities of daily living, hospital acquired condition, wound healing, and discharge disposition.

Data syntheses were performed using methods consistent with those outlined in the AHRQ Methods Guide (Methods Guide for Effectiveness and Comparative Effectiveness Reviews Effective Health Care Program [(ahrq.gov)].

\section{Results}

A total of 17 studies (6 SRs and 11 RCTs) met eligibility criteria for inclusion. Existing SRs found that patients screened or diagnosed with malnutrition (using various measurement tools) may be at increased risk of hospital mortality compared to well-nourished patients (Strength of evidence [SOE]: Low). This association was observed among patients hospitalized for acute medical conditions, heart failure, and cirrhosis. Malnutrition (diagnosed using SGA) was also independently associated with prolonged hospital length of stay among patients hospitalized with acute medical conditions (SOE: Moderate) or cancer (SOE: Low). Finally, malnutrition 
(diagnosed using MNA or screened using NRS-2002) was found to be associated with increased hospital acquired conditions among patients hospitalized due to traumatic injury or acute medical conditions compared to well-nourished patients (SOE: Low).

To assess clinical utility of measurement tools we sought to identify prospectively controlled studies in which some patients were screened or assessed for malnutrition while other patients were either (1) not screened or assessed or (2) assessed with a reference standard (i.e., imaging or SGA). However, we identified no studies meeting these criteria.

We identified 11 RCTs indicating that some interventions improve clinical outcomes among malnourished patients (screened at risk or diagnosed with malnutrition using commonly available measurement tools). Specifically, we found that hospital-initiated malnutrition interventions (i.e., specialized nutrition care and increased protein/calorie provision) likely reduce mortality compared to usual care (SOE: moderate); these interventions may also improve quality of life (SOE: Low). However, evidence was insufficient or showed no difference for other outcomes (length of stay, activities of daily living, discharge disposition, hospital acquired conditions, or adverse events).

\section{Limitations and Suggestions for Future Research}

No studies met criteria to address clinical effectiveness of measurement tools. Eleven RCTs assessed hospital-initiated malnutrition interventions. Furthermore, although SRs assessing the association between malnutrition and clinical outcomes included a combined 80 studies, only 43 used a known tool to measure malnutrition and could be included for this review.

This evidence base reveals several shortcomings of the published literature on malnutrition in hospitalized patients. First, only a relatively small number of studies used commonly available measurement tools to identify malnutrition. Instead, many studies identified malnutrition using only biometric measures, such as serum albumin levels, body mass index, and weight. ${ }^{1,2}$ Future studies assessing the impact of malnutrition on outcomes or evaluating malnutrition interventions should use known tools to establish malnutrition status.

The absence of studies addressing the clinical utility (effectiveness) of measurement tools for nutrition screening and diagnostic assessment (Key Question 2) does not necessarily imply that these tools are ineffective. Instead, it highlights two important knowledge gaps in current literature. First, is the need for controlled studies assessing their effectiveness in hospitalized adults. Understanding downstream consequences of malnutrition screening, including subsequent diagnostic assessment, management, and clinical outcomes is extremely important as US hospitals are mandated to provide nutrition screening for all hospitalized patients within 24 hours of admission. Further research could also support alignment of screening efforts with similar tools across different institutions.

Second, is the need to establish an accepted reference gold standard for diagnosing malnutrition in hospitalized patients. Through discussions with our Technical Expert Panel (TEP), we recognized that there currently is no universally agreed upon gold standard for malnutrition assessment and measurement. For the purposes of this report, we selected, with input from our TEP and subject matter experts, imaging modalities to quantify and evalute body composition (i.e., muscle and adipose tissues) as the gold standard and SGA as a semi-gold standard for classifying malnutrition. However, use of imaging specifically to assess malnutrition is infrequent and has important limitations, including cost, radiation exposure, and need for serial studies. Consensus regarding objective measures to define a gold standard for diagnosing malnutrition are critical to advance clinical care and research. 
Finally, studies addressing efficacy of malnutrition-focused interventions only addressed specialized nutrition care (consultation with a dietitian to set goals for protein and calorie intake) or increased protein/calorie provision. These studies had several shortcomings, including high risk of bias and poor reporting of adverse events. These limitations, along with inconsistencies in the findings for some outcomes and lack of precision for others, downgraded the overall strength of the evidence to low or insufficient for most outcomes. Future studies need to clearly indicate any harms associated with treatment. 


\section{References}

1. Bharadwaj S, Ginoya S, Tandon P, et al. Malnutrition: laboratory markers vs nutritional assessment. Gastroenterol Rep (Oxf). 2016 Nov;4(4):272-80. Epub 2016 May 11. http://dx.doi.org/10.1093/gastro/gow013. PMID: 27174435.
2. Barone M. Is the use of the BMI alone sufficient to diagnose malnutrition in both male and female adults? Clin Nutr. 2018 Oct;37(5):1771.

http://dx.doi.org/10.1016/j.clnu.2018.07.003.

PMID: 30055866. 


\section{Introduction}

\section{Background}

Malnutrition among hospitalized patients remains a serious issue affecting more than 30 percent of hospitalized patients in the United States. ${ }^{1}$ According to the American Society for Parenteral and Enteral Nutrition (ASPEN), malnutrition results from a "combination of varying degrees of overnutrition or undernutrition with or without inflammatory activity that leads to a change in body composition and diminished function." "2 The World Health Organization (WHO) categorizes malnutrition into two broad groups - undernutrition and overweight or obesity. ${ }^{3}$ Malnutrition in both groups results from inadequate intake of macro and micronutrients, leading to nutritional imbalances and adverse body composition changes. Malnutrition resulting from undernutrition manifests in severe weight loss and muscle wasting. The etiology of malnutrition is heterogeneous, and can result from chronic starvation (e.g., anorexia nervosa), acute or chronic illness (e.g., certain cancers, sarcopenic obesity, major infections), and injury (e.g., burns, head trauma). Factors such as advanced age, immobilization, and low income can increase the risk of malnutrition.

Malnutrition is associated with high mortality and morbidity, functional decline, prolonged hospital stays, and increased healthcare costs. ${ }^{4}$ An Agency for Healthcare Research and Quality (AHRQ) Healthcare Cost and Utilization Project (HCUP) Statistical Brief reported 30-day all cause readmission was nearly 50 percent higher among patients with malnutrition compared to patients with no associated malnutrition. ${ }^{5,6}$

In 1995, the Joint Commission mandated universal screening of all hospitalized patients for malnutrition as part of general admission processes. ${ }^{7}$ Findings from a national survey of hospitalbased professionals representing ASPEN and other professional societies in the United States suggests high compliance with the screening mandate. The findings found 36.7 percent of respondents reporting completing nutrition screening at admission, 50.8 percent reporting doing so within 24 hours, and 69 percent reporting documenting the findings in the medical record. ${ }^{7}$ However, while 71 percent of respondents indicated that screening information from medical records led to further nutritional assessment, only 26 percent reported that diagnosis of malnutrition was based on nutrition assessment. In a more recent survey of ASPEN members, 89 percent reported that a dietitian completed a nutrition assessment once an adult patient screened at high risk for malnutrition. ${ }^{8}$ While these findings are positive, they still suggest opportunities for hospitals to improve their processes for identifying, diagnosing, and documenting malnutrition.

No national benchmarking of malnutrition in acute care hospitals currently exists in the United States. ${ }^{9}$ Recently, the Academy of Nutrition and Dietetics (AND) along with Avalere Health developed a composite measure for malnutrition now under consideration at the Centers for Medicare \& Medicaid Services (CMS). ${ }^{10}$ The measure, titled Global Malnutrition Composite Score, is intended to assess provider performance on following the recommended malnutrition workflow, which includes (1) completion of screening of all hospital patients, (2) further assessment of patients at risk for malnutrition, (3) appropriate documentation of malnutrition diagnosis, and (4) implementation of a nutrition care plan. This measure is specific to older adults (ages 65 and older) who are at higher risk of facing poor clinical outcomes and is intended for use in electronic health records. 
For the implementation of these or other quality measures of malnutrition to be considered, screening and nutrition assessment must first be standardized across hospitals to accurately identify and monitor rates of malnutrition. However, variations in definitions and tools used to screen and diagnose malnutrition have made it difficult for hospitals to standardize this process. ${ }^{11,12}$ National survey data indicate that only 38 percent of hospital professionals report using a recognized tool to screen for malnutrition, and only 23 percent report using one for further diagnostic assessment. ${ }^{7}$ Currently, more than 20 different tools exist to assess nutritional status. Some tools were specifically developed to screen nutritional risk, while others are intended for diagnostic assessment. As in other fields, screening tools are designed to be sensitive but not specific, in order to identify patients "at risk" for malnutrition, who then warrant further diagnostic assessment. Then, a second assessment tool is utilized to formally diagnose patients with malnutrition who warrant targeted treatments.

Determining what tool to use can be challenging as the clinical literature often blurs the distinction between screening and diagnostic assessment by using tools interchangeably. ${ }^{12,13}$ Unlike other commonly used screening tools for other clinical conditions, the difference between screening and diagnostic assessment for malnutrition is not about the degree of invasiveness, as both are questionnaires completed at patient bedside with incorporation of limited clinical data. The distinguishing feature is that screening tools are intended to be short and simple, in order to facilitate widespread use at the bedside by nurses and dietary technicians, while diagnostic assessments typically require more detailed assessment by a registered dietitian. Table 1 describes commonly available tools. As shown, there is significant overlap in the components across tools, and crossover between screening and diagnostic assessments, further blurring these categories. Screening tools are intended to identify "at risk" patients, who can then undergo formal diagnosis using a diagnostic tool. However, both in clinical practice and research, screening and diagnostic tools have often been used interchangeably, and patients without a formal diagnosis of malnutrition often receive interventions for malnutrition.

Table 1. Commonly available measurement tools and criteria for assessing nutritional status

\begin{tabular}{|c|c|c|c|c|}
\hline Tool & Use & Population & Setting & $\begin{array}{l}\text { Screening/Diagnosis } \\
\text { Components }\end{array}$ \\
\hline $\begin{array}{l}\text { Malnutrition Screening } \\
\text { Tool (MST) }\end{array}$ & Screening & $\begin{array}{l}\text { Adults (includes } \\
\text { the elderly) }\end{array}$ & $\begin{array}{l}\text { Acute care, inpatient, } \\
\text { outpatient, residential } \\
\text { aged care facilities }\end{array}$ & $\begin{array}{l}\text { Recent weight loss; recent poor } \\
\text { food/nutrient intake }\end{array}$ \\
\hline $\begin{array}{l}\text { Malnutrition Universal } \\
\text { Screening Tool (MUST) }\end{array}$ & Screening & Adults & $\begin{array}{l}\text { Acute care and } \\
\text { community }\end{array}$ & $\begin{array}{l}\text { BMI, weight loss, acute disease } \\
\text { effect score }\end{array}$ \\
\hline $\begin{array}{l}\text { Nutritional Risk } \\
\text { Screening } 2002 \\
\text { (NRS-2002) }\end{array}$ & Screening & Adults & Acute care & $\begin{array}{l}\text { Recent weight loss, recent poor } \\
\text { food/nutrient intake, BMI, and } \\
\text { severity of disease }\end{array}$ \\
\hline $\begin{array}{l}\text { Nutritional Risk Index } \\
\text { (NRI) }\end{array}$ & Screening & $\begin{array}{l}\text { Adults (includes } \\
\text { the elderly) }\end{array}$ & Hospital & Current and usual weight \\
\hline $\begin{array}{l}\text { Mini Nutritional } \\
\text { Assessment-Short } \\
\text { Form (MNA-SF) } \\
\end{array}$ & Screening & Geriatric & $\begin{array}{l}\text { Acute care, } \\
\text { community, rehab, } \\
\text { long-term care }\end{array}$ & $\begin{array}{l}\text { Recent weight loss and diet } \\
\text { history }\end{array}$ \\
\hline $\begin{array}{l}\text { Nutrition Risk in } \\
\text { Critically III (NUTRIC) } \\
\text { score }\end{array}$ & Screening & Adults (critically ill) & ICU & $\begin{array}{l}\text { Age, severity of illness, co- } \\
\text { morbidities, days in from } \\
\text { hospital to ICU }\end{array}$ \\
\hline $\begin{array}{l}\text { Short Nutritional } \\
\text { Assessment } \\
\text { Questionnaire (SNAQ) }\end{array}$ & Screening & Adult & Hospital outpatients & Weight loss and dietary intake \\
\hline
\end{tabular}




\begin{tabular}{|c|c|c|c|c|}
\hline Tool & Use & Population & Setting & $\begin{array}{l}\text { Screening/Diagnosis } \\
\text { Components }\end{array}$ \\
\hline $\begin{array}{l}\text { Adult Malnutrition } \\
\text { Consensus } \\
\text { characteristics (AMC) })^{14}\end{array}$ & Diagnosis & Adult & Hospital & $\begin{array}{l}\text { Weight loss, energy intake, } \\
\text { body fat, muscle mass, fluid } \\
\text { accumulation, and grip strength }\end{array}$ \\
\hline $\begin{array}{l}\text { Subjective Global } \\
\text { Assessment (SGA) }\end{array}$ & Diagnosis & $\begin{array}{l}\text { Adults (includes } \\
\text { surgical patients, } \\
\text { geriatric, oncology, } \\
\text { and renal) }\end{array}$ & $\begin{array}{l}\text { Acute care, rehab, } \\
\text { residential or } \\
\text { community }\end{array}$ & $\begin{array}{l}\text { Medical history (weight, intake, } \\
\text { symptoms, functional capacity, } \\
\text { metabolic demand) and } \\
\text { physical exam }\end{array}$ \\
\hline $\begin{array}{l}\text { Patient Generated } \\
\text { Subjective Global } \\
\text { Assessment (PG-SGA) }\end{array}$ & Diagnosis & $\begin{array}{l}\text { Adults (includes } \\
\text { oncology, renal } \\
\text { and stroke) }\end{array}$ & Acute care & $\begin{array}{l}\text { Medical history (weight, intake, } \\
\text { gastro-intestinal symptoms, } \\
\text { functional capacity) and } \\
\text { physical exam }\end{array}$ \\
\hline $\begin{array}{l}\text { Mini Nutritional } \\
\text { Assessment (MNA) }\end{array}$ & Diagnosis & Geriatric & $\begin{array}{l}\text { Acute care, } \\
\text { community, rehab, } \\
\text { long-term care }\end{array}$ & $\begin{array}{l}\text { Diet history, anthropometry, } \\
\text { medical history, and functional } \\
\text { history }\end{array}$ \\
\hline $\begin{array}{l}\text { Academy of Nutrition } \\
\text { and Dietetics and } \\
\text { American Society for } \\
\text { Parenteral and Enteral } \\
\text { Nutrition (AND and } \\
\text { ASPEN) Malnutrition } \\
\text { Consensus Criteria } \\
(\text { MCC) }\end{array}$ & Diagnosis & Adults & Hospital & $\begin{array}{l}\text { Insufficient energy intake, } \\
\text { weight loss, loss of muscle } \\
\text { mass, loss of subcutaneous fat, } \\
\text { localized or generalized fluid } \\
\text { accumulation (that may } \\
\text { sometimes mask weight loss, } \\
\text { diminished functional status as } \\
\text { measured by handgrip strength }\end{array}$ \\
\hline $\begin{array}{l}\text { Global Leadership } \\
\text { Initiative on Malnutrition } \\
(\text { GLIM })^{16}\end{array}$ & Diagnosis & Adults & Hospital & $\begin{array}{l}\text { AND-ASPEN's criteria, and } \\
\text { etiologic influences (reduced } \\
\text { food intake, hypercatabolic } \\
\text { burden of disease) and } \\
\text { phenotypic presentations (non- } \\
\text { volitional weight loss, low body } \\
\text { BMI, low skeletal muscle mass) } \\
\text { of malnutrition }\end{array}$ \\
\hline $\begin{array}{l}\text { Nutrition Focused } \\
\text { Physical Exam } \\
(\text { NPFE) })^{17}\end{array}$ & Diagnosis & Adults & Hospital & $\begin{array}{l}\text { Insufficient energy intake, } \\
\text { weight loss, loss of muscle } \\
\text { mass, loss of subcutaneous fat, } \\
\text { localized or generalized fluid } \\
\text { accumulation (that may } \\
\text { sometimes mask weight loss), } \\
\text { diminished functional status as } \\
\text { measured by hand-grip } \\
\text { strength }\end{array}$ \\
\hline
\end{tabular}

Source: Mueller et al. $2011^{18}$ unless otherwise specified

$\mathrm{BMI}=$ body mass index; ICU $=$ intensive care unit

To promote consistency in assessing malnutrition, AND and ASPEN jointly published a set of criteria for hospitals to use for diagnosing and documenting malnutrition in hospitalized patients. The criteria focus on the following six characteristics: insufficient energy intake, weight loss, loss of muscle mass, loss of subcutaneous fat, localized or generalized fluid accumulation (that may sometimes mask weight loss), and diminished functional status as measured by handgrip strength. ${ }^{15}$ These criteria are the basis of the Adult Malnutrition Consensus diagnostic tool and the Nutrition Focused Physical Exam listed in Table 1, and are similar to the diagnostic criteria used in other tools, such as Subjective Global Assessment (SGA).

In 2016, the Global Leadership Institute on Malnutrition (GLIM) taskforce convened to develop a universal framework for assessing malnutrition. ${ }^{16}$ The GLIM taskforce 
recommendations were published in 2019 and include the following two-step approach to identify malnutrition: 1) screening for malnutrition using a valid tool, followed by 2) formal diagnostic assessment. The taskforce produced consensus-based criteria for formal assessment that incorporates AND-ASPEN's criteria, and includes both etiologic influences (reduced food intake, hypercatabolic burden of disease) and phenotypic presentations (non-volitional weight loss, low body mass index (BMI), low skeletal muscle mass) of malnutrition. To be diagnosed with malnutrition, patients must have at least one etiologic criterion and one phenotypic criterion. A diagnosis of severe malnutrition depends upon the severity of the phenotypic presentation. The GLIM recommendations have yet to be validated but represent the current consensus of experts in the field. ${ }^{19}$

Diagnosis of malnutrition in hospitalized patients allows clinicians to target appropriate nutrition-focused interventions. While some interventions, such as nutritional assessments, initiating oral nutrition supplements (ONS), diet changes, visits with registered dietitians, and tracking biomarkers, may be uniformly applied across populations, others, such as initiating enteral nutrition (EN) or parenteral nutrition (PN), may only be appropriate in specific cases. ASPEN and the American College of Gastroenterology (ACG) have published clinical practice guidelines addressing the initiation of EN and $\mathrm{PN}$ in adult hospitalized patients. ${ }^{11,20}$ Since specific interventions for treatment of malnutrition have risks (e.g., risk of blood-stream infections with PN or complications from gastrostomy tube placement), identifying the appropriate context in which treatments are effective or harmful is important.

\section{Purpose and Scope of the Systematic Review}

In fiscal year 2020, Congress requested that AHRQ convene a panel of experts charged with developing quality measures for malnutrition-related hospital readmissions. These measures would support assigning accountability for the assessment and treatment of malnutrition in hospitalized adults, with an emphasis on the needs of older frail adults. This systematic review is intended to support the efforts of the panel by identifying and synthesizing published literature on the association between malnutrition and clinical outcomes among hospitalized patients, particularly those who may be at greater risk of malnutrition. This review also evaluates the effectiveness of screening and/or diagnostic assessment of malnutrition on clinical outcomes and the impact of hospital-initiated interventions for patients diagnosed with malnutrition. Findings from this review are intended to inform potential development of quality measures in malnourished hospital adults. 


\section{Organization of This Report}

In the remaining three chapters of this report, we describe the methods for this systematic review, present results, and discuss overall findings. Within the Results chapter, we provide results of the literature searches, screening procedures, descriptions of included studies, key points, detailed syntheses of the findings, and strength-of-evidence tables. The Discussion chapter reviews key findings and strength of evidence, places findings in the context of clinical practice, examines the general applicability of the findings, discusses implications for decision making, describes limitations of the systematic review process and the evidence base, and identifies knowledge gaps requiring further research. The main body of the report is followed by nine appendixes: Appendix A. Search Strategy; Appendix B. Methods; Appendix C. Excluded Studies; Appendix D. Characteristics of Included Studies; Appendix E. Risk of Bias; Appendix F. Results From Included Studies; Appendix G. Forest Plots of Additional Analysis; Appendix H. Additional Information for Key Question 1; and Appendix I. Appendix References. 


\section{Methods}

\section{Review Approach}

This Comparative Effectiveness Review follows the methods suggested in the Agency for Healthcare Research and Quality (AHRQ) "Methods Guide for Effectiveness and Comparative Effectiveness Reviews" (hereafter, "AHRQ Methods Guide"). ${ }^{21}$ Our methods are summarized in this section; for additional details, see the review protocol posted on the AHRQ Effective Health Care Program website (www.effectivehealthcare.ahrq.gov).

\section{Role of the Technical Expert Panel}

We convened a 5-member Technical Expert Panel (TEP) to provide guidance and feedback throughout systematic review (SR) development. The TEP included key stakeholders with expertise in malnutrition-related translational, epidemiologic, and clinical research as well as policy development, guideline creation, clinical care coordination, and quality metric development. (See the front matter of the report for TEP names, credentials, and affiliations). Several TEP members had first-hand experience with caring for hospitalized patients with malnutrition, utilizing available measurement tools, and administering various treatments. We sought TEP feedback on the scope and protocol of the review through real-time video-based oneon-one interviews, group meetings, and email communication. Subject matter experts (SMEs) were sometimes included in group meetings to facilitate discussions and help reach consensus when needed.

Input from the TEP informed the measurement tools selected for inclusion and patient populations included for each Key Question (KQ). Insights from the TEP (as well as SMEs) were particularly critical in selecting an appropriate comparator or gold standard for screening and diagnostic assessments (KQ 2). Ultimately, TEP members agreed that currently, no nutritional biomarker or imaging technique is widely accepted as a gold standard to use to validate screening or diagnostic tools. However, radiographic imaging and Subjective Global Assessment (SGA) were felt to be the most highly respected and commonly used in the field. Therefore, these were identified as "semi-gold" standards and were specified as appropriate comparators for determining tool effectiveness in KQ 2. Finally, we sought input from TEP members on methodologic approaches to best inform creation of quality metrics. One TEP member with expertise in this area provided useful context to ensure extraction of relevant data, such as categorization of patient populations, malnutrition definitions, and outcome specifications.

\section{Key Questions}

The intent of the following KQs is to identify evidence to inform the development of quality measures related to malnutrition in hospitalized adult patients. These questions underpin the pathway of care linking patients at risk of malnutrition to clinical outcomes.

Key Question 1. What is the association between malnutrition and clinical outcomes among hospitalized patients?

\section{a. How do outcomes vary depending on measures or tools used to detect malnutrition?}


b. Are patient-related risk factors, such as increased age or certain preexisting health conditions, associated with poorer clinical outcomes?

Key Question 2. What is the effectiveness of screening or diagnostic assessment for malnutrition among hospitalized adults?

a. In studies that report on clinical outcomes, what is the accuracy of screening or diagnostic tools for malnutrition?

b. In studies that report on clinical outcomes, what is the effectiveness of screening or diagnostic tools on measures of nutrition (nutritional stores)?

c. What is the impact of the use of screening or diagnostic tools on clinical outcomes?

Key Question 3. Among patients diagnosed with malnutrition, what is the effectiveness of hospital-initiated interventions used to treat malnutrition on clinical outcomes?

\section{Analytic Framework}

Figure 1 presents the analytical framework, which visually presents the pathway of hospitalized patients who undergo screening for malnutrition. It depicts movement along several exposures beginning with screening, moving to further assessment and treatment, and ultimately ending with clinical outcomes. Figure 2 shows the overall schematic of potential study designs for this review, incorporating the process of screening, followed by diagnosis. It also depicts the control and comparator groups with appropriate reference standards as determined by the TEP.

Figure 1. Analytic framework for malnutrition in hospitalized adults

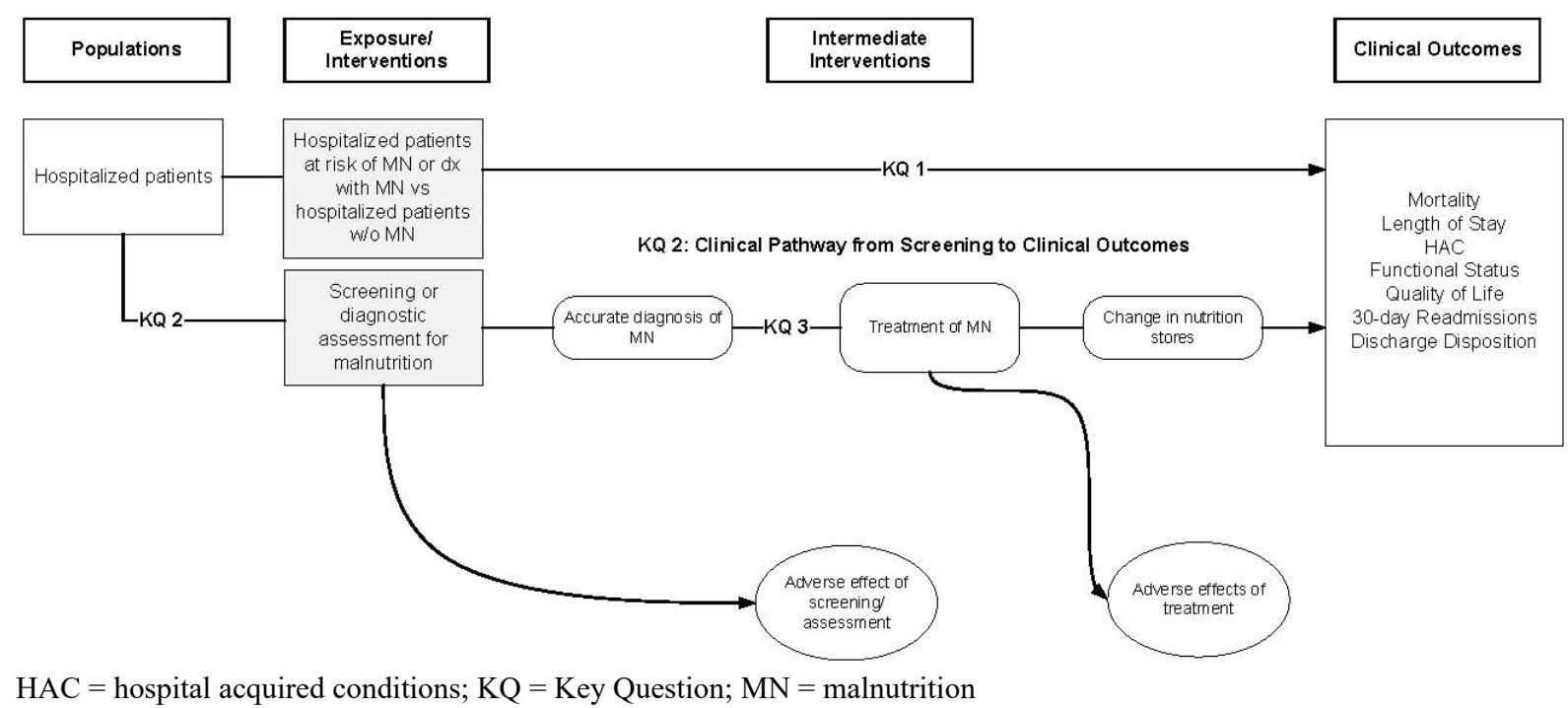


Figure 2. Study design schematic

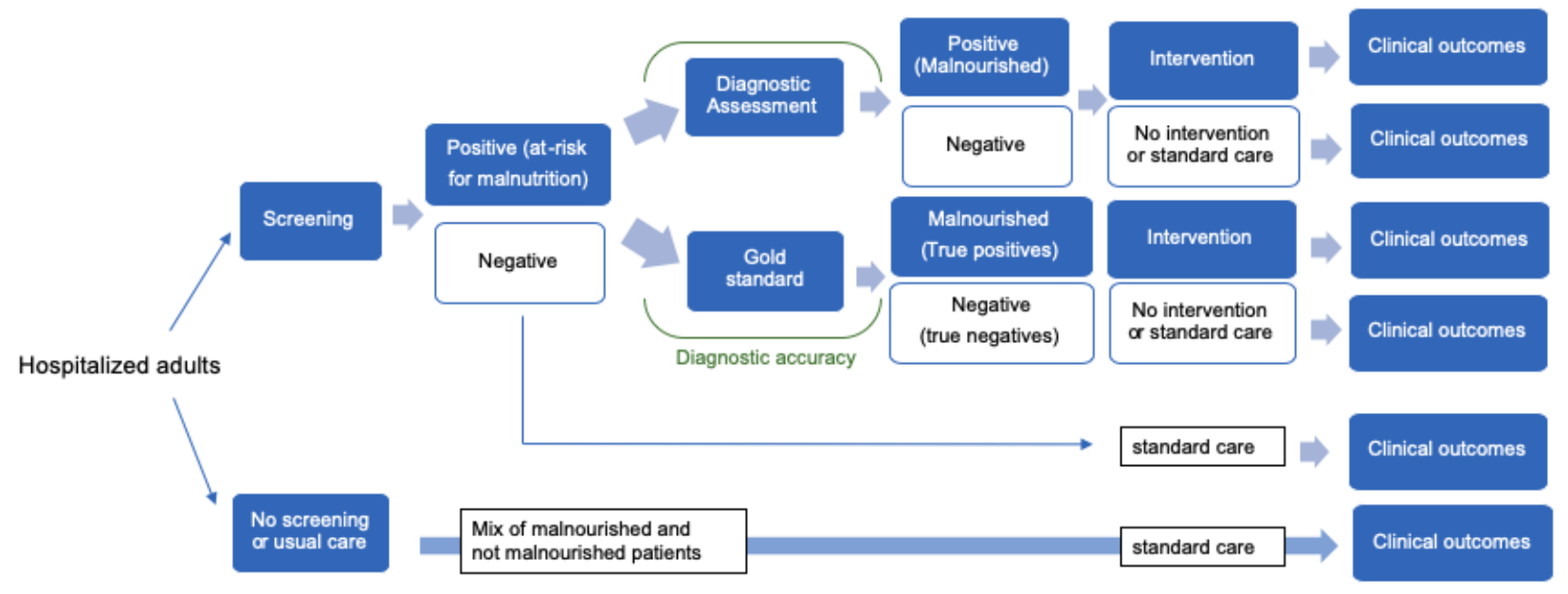

\section{Study Selection}

To identify articles relevant to each KQ, Evidence-based Practice Center (EPC) librarians conducted a comprehensive literature search including studies from January 2000 to June 3, 2021, searching MEDLINE ${ }^{\circledR}$, Embase.com, Cochrane Central Register of Controlled Trials, and ClinicalTrials.gov. They also searched for gray literature in websites of the following organizations: Centers for Disease Control and Prevention (CDC), Medscape, National Academy of Medicine, the United States Food and Drug Administration (FDA), AHRQ, American Society for Parenteral and Enteral Nutrition (ASPEN), and Academy of Nutrition and Dietetics (AND), and hand-searched reference lists of relevant studies and searched for unpublished studies. Literature searches were updated during the public comment and peer review period to capture any new publications. Search strategies are available in Appendix A.

Studies were selected for inclusion using pre-established population, intervention, comparator, outcome, timing, and setting specifications (Table 2). Literature screening was performed in duplicate using the database Distiller SR (Evidence Partners, Ottawa, Canada). Literature search results were initially screened by title for relevancy. Relevant abstracts were screened against the inclusion and exclusion criteria in duplicate. Studies that met inclusion criteria were retrieved in full and the full study was screened again in duplicate against inclusion and exclusion criteria. Disagreements were resolved by consensus discussion between the two original screeners. Further details about study selection processes are provided in Appendix B. 
Table 2. PICOTS (population, intervention, comparator, outcome, timing, setting)

\begin{tabular}{|c|c|}
\hline Category & Definition \\
\hline Population & $\begin{array}{l}\text { Key Question } 1 \text { and 2: Hospitalized adults aged } 18 \text { years or older. } \\
\text { Key Question 1b: Subgroups include adults with no risk of malnutrition, adults with risk of } \\
\text { malnutrition, and adults with baseline malnutrition. Risk factors of interest to this report include: } \\
\text { - } \quad \text { Older patients (>65 years) } \\
\text { - } \quad \text { Racial and ethnic minorities } \\
\text { - } \quad \text { Low income (e.g., Medicaid beneficiaries) } \\
\text { - Patients with malignancy } \\
\text { - } \quad \text { Patients with gastrointestinal disease and subsequent malabsorption, including ulcerative } \\
\text { - } \quad \text { Patitis and Crohn's disease } \\
\text { - Patients with stroke } \\
\text { - Patients with chronic kidney disease } \\
\text { - Patients with dementia } \\
\text { - Patients with critical illness } \\
\text { - Sepsis/infection } \\
\text { Key Question 3: Adults diagnosed with protein-energy malnutrition. }{ }^{*}\end{array}$ \\
\hline & $\begin{array}{l}\text { Key Question 1: Positive screening for nutrition risk and/or diagnosis of malnutrition vs no } \\
\text { malnutrition. } \\
\text { Key Question 2: Malnutrition measurement tools (utilized within the U.S., Australia, New Zealand, } \\
\text { Canada, and Europe). Examples of tools of interest include: } \\
\text { Screening } \\
\text { - } \quad \text { Malnutrition Screening Tool (MST) } \\
\text { - } \quad \text { Malnutrition Universal Screening Tool (MUST) } \\
\text { - } \quad \text { Nutritional Risk Index (NRI) } \\
\text { - } \quad \text { Nutrition Risk in Critically III (NUTRIC) score } \\
\text { Diagnostic Assessment } \\
\text { - } \quad \text { Subjective Global Assessment (SGA) } \\
\text { - } \quad \text { Patient Generated Subjective Global Assessment (PS-SGA) } \\
\text { - } \quad \text { Mini Nutritional Assessment (MNA) } \\
\text { - } \quad \text { AND (Academy of Nutrition and Dietetics)-ASPEN (American Society for Parenteral and } \\
\text { - } \quad \text { Gnteral Nutrition) Malnutrition Consensus Criteria (MCC) } \\
\text { Key Question 3: Hospital-initiated malnutrition interventions. Examples of interventions include: } \\
\text { - } \quad \text { Parenteral nutrition } \\
\text { - } \quad \text { Enteral nutrition } \\
\text { - Oral nutrition supplements }\end{array}$ \\
\hline Comparators & $\begin{array}{l}\text { Key Question 1: Hospitalized patients without malnutrition, or direct comparisons of different } \\
\text { definitions of malnutrition. } \\
\text { Key Questions 2: For screening tools: no screening. For diagnostic assessments: no assessment } \\
\text { or imaging modalities to assess body composition and muscle mass (i.e. nutrition stores) or SGA } \\
\text { as the reference standard. } \\
\text { Key Question 3: Usual care or another hospital-initiated malnutrition-related intervention. }\end{array}$ \\
\hline
\end{tabular}




\begin{tabular}{|c|c|}
\hline Category & Definition \\
\hline Outcomes & 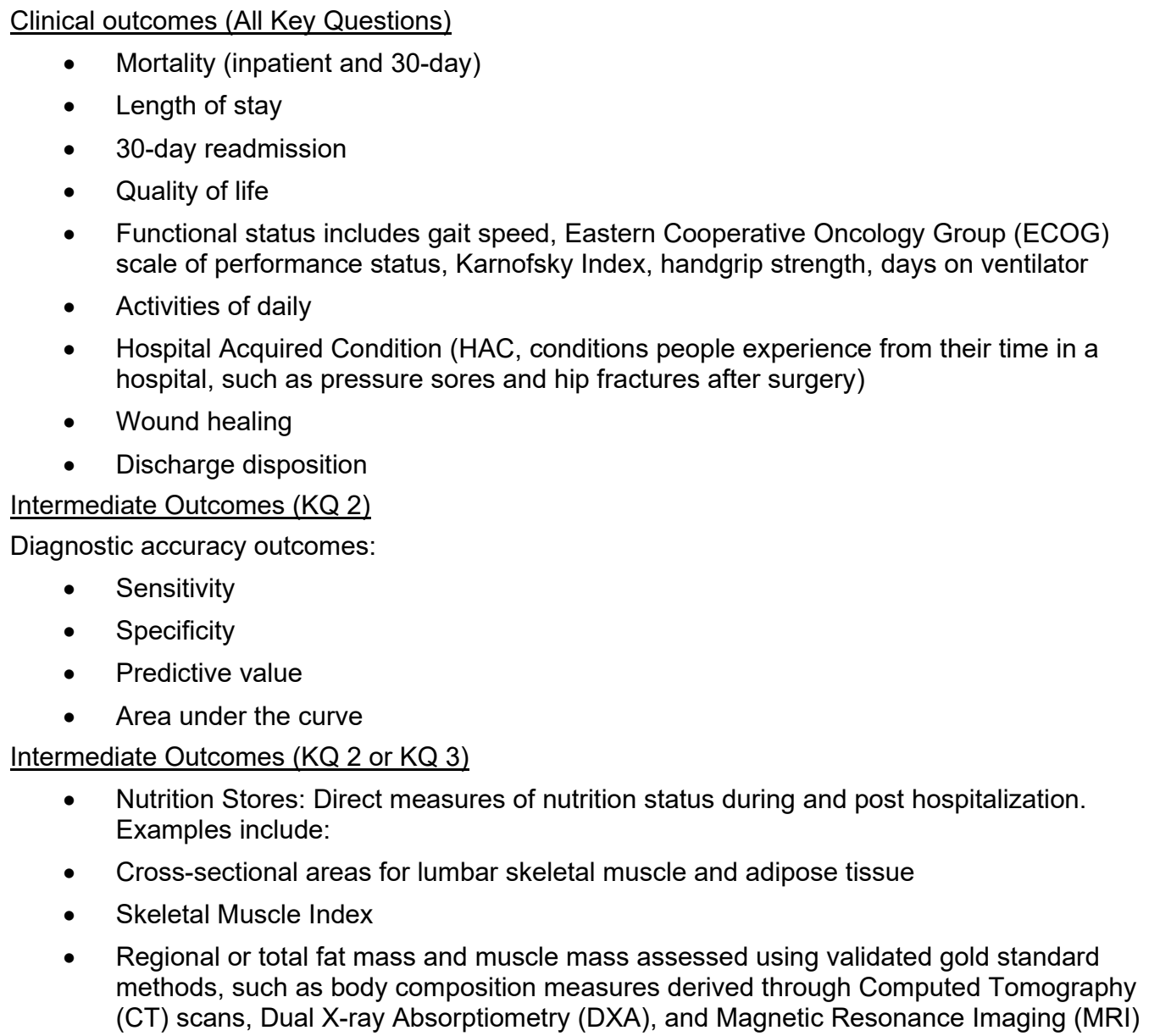 \\
\hline Timing & Up to 30 days post-discharge. \\
\hline Setting & Acute care hospitalizations. \\
\hline Study Design & $\begin{array}{l}\text { Key Question 1: Systematic reviews of relevant study designs (RCTs, prospective cohort trials, or } \\
\text { cross-sectional studies). } \\
\quad \text { We did not include systematic reviews of isolated micro-nutrient deficiencies, such as } \\
\text { cobalamin or iron deficiencies } \\
\text { Key Question 2: Randomized or non-randomized comparative trials; retrospective studies were } \\
\text { not considered for inclusion for this Key Question as these types of study designs are subject to } \\
\text { biases that reduce the reliability of the findings. } \\
\text { Key Question 3: RCTs. }\end{array}$ \\
\hline
\end{tabular}

* Due to the paucity of literature identified for KQ 3 in which patients were diagnosed with malnutrition using diagnostic assessment tools (such as SGA or MNA), we accepted studies in which patients were identified as at-risk of malnutrition using cutoff scores on screening tools (such as NRS-2002 and MUST).

$\mathrm{KQ}=$ Key Question; RCT = randomized controlled trial

\section{Data Extraction}

Data were extracted from included studies into standardized forms in Microsoft Word. All relevant study-level and patient-level characteristics were extracted (author, year, study design, setting, country, sample size, eligibility criteria, intervention, comparator, screening/diagnostic instrument, population characteristics, clinical conditions, and results). For SRs addressing Key Question 1 (KQ 1), we also extracted information about the search strategy, study selection, and 
final number of included studies. For studies that contained data points displayed in survival curves or other figures, we used WebPlotDigitizer v 4.4 to extract relevant data. ${ }^{22}$ A single trained reviewer extracted the relevant data from each included article into evidence tables. A second member of the team reviewed all data extractions for completeness and accuracy. Discrepancies were resolved through team discussion.

\section{Risk of Bias Assessment}

Risk of bias (ROB) of individual studies and existing SRs was assessed by an independent reviewer and quality checked by a second reviewer. Discrepancies in ROB were addressed through consensus discussion.

\section{Primary Studies}

To judge the ROB of the individual randomized controlled trials (RCTs), we used the Cochrane Risk of Bias 2.0 tool (ROB 2). ${ }^{23}$ This tool assesses ROB along the following domains: randomization process, deviations from intended interventions, attrition, and reporting bias. Overall summary ROB assessments for each study were classified as low risk of bias, some concerns, or high risk of bias based upon the collective ROB inherent in each domain and confidence that results are believable given the study's limitations.

Observational studies were assessed using the Risk of Bias in Non-randomized Studies (ROBINS-I) tool. ${ }^{24}$ The ROBINS-I tool measures potential bias along the following domains: confounding, selection of participants, classification of intervention, deviation from intervention, missing data, measurement of outcomes, and reported results. The categories for risk of bias judgements are low risk, moderate risk, serious risk, and critical risk of bias. We did not exclude studies rated high ROB a priori, but did consider them the least reliable when synthesizing the evidence, particularly when discrepancies among studies were present.

\section{Systematic Reviews}

We assessed ROB of SRs included for KQ 1 using the Risk of Bias in Systematic Reviews (ROBIS) tool. ${ }^{25}$ This tool covers four domains which may introduce bias: study eligibility criteria, identification and selection of studies, data collection and study appraisal, and data synthesis and findings. The instrument includes signaling questions to assess potential bias in each domain. We classified the overall ROB for each review as low or high. For primary studies included in the SRs, we relied on the quality ratings or ROB assessments performed in the systematic review if the review used a standardized method for assessing quality (e.g., Newcastle-Ottawa Scale, Cochrane tool).

We rated SRs low ROB if they used multiple sources in the literature search, applied predefined inclusion and exclusion criteria, assessed study quality using an appropriate tool, used methods to reduce errors in data abstraction and quality rating (e.g., multiple independent reviewers), used appropriate methods for evidence synthesis (qualitative or quantitative), and used an explicit system for considering the body of evidence (that included major domains of strength of evidence [e.g., risk of bias, consistency, precision, and directness]). If SRs were found to have shortcomings in one or more of these areas, we only included the SR if we determined it was possible to address the shortcomings (e.g., by assessing the quality of primary studies ourselves or independently determining the strength of evidence from the information provided in the review). For example, if the methods used by SR authors to assess the risk of bias 
of individual studies were unclear, we assessed the quality of individual studies ourselves, using methods described previously.

\section{Data Synthesis and Analysis}

We summarized all included studies in narrative form and in summary tables presenting key features of study populations, design, intervention, outcomes, setting (including geographic location), and results.

We conducted meta-analysis, whenever appropriate (i.e., $\geq 2$ studies addressing the same populations, interventions, comparators, outcomes, timings, and settings (PICOTS) and providing point estimates and dispersion measures), to quantitatively summarize study findings. Relative risk and corresponding 95 percent confidence intervals were extracted or calculated for binary outcomes. For continuous outcomes, we used mean differences weighted by sample size. If primary studies reported a continuous outcome using different scales (e.g., quality of life), we used standardized mean differences and converted the direction of all measures (e.g., all higher score represents better outcome). For continuous outcomes, we used means and standard deviations to conduct meta-analyses; if medians were extracted, they were converted to means using the quantile-estimation method. ${ }^{26} \mathrm{We}$ conducted all meta-analyses using the DerSimonian and Laird random effect model with Hartung-Knapp-Sidik-Jonkman variance correction. ${ }^{27,28}$ Meta-analyses were performed by transferring data from standardized extraction forms into Microsoft Excel and importing study-level data into Stata 13_(StataCorp. 2013. Stata Statistical Software: Release 13. College Station, TX: StataCorp LP). ${ }^{29}$

We used the $\mathrm{I}^{2}$ test to evaluate statistical heterogeneity; an $\mathrm{I}^{2}$ of 50 percent or more indicated the presence of substantial heterogeneity. We performed subgroup analysis to assess if treatment effects varied by age, definition of malnutrition, type of treatment, follow-up time, and comorbid conditions. When meta-analysis was not possible (due to limitations in number of studies or reported data) or judged to be inappropriate (e.g., due to large clinical heterogeneity in population and treatments), data were synthesized using a descriptive, narrative review approach.

For KQ 1, we reported meta-analytic findings from included SRs if we judged appropriate pooling methods were used (e.g., random effects model in the presence of statistical heterogeneity). When SR authors did not perform meta-analysis due to clinical or statistical heterogeneity, we qualitatively synthesized reported findings for outcomes of interest. Findings for all outcomes of interest were synthesized and reported by nutritional screening or assessment tool. We did not conduct updated meta-analysis or qualitative summary of evidence from newer studies (e.g., published after publication of the review). However, we summarized key characteristics of both (1) relevant primary studies identified, but not captured in included SRs, and (2) relevant studies published after included SRs in Appendix H.

\section{Grading the Strength of the Body of Evidence}

We appraised the strength of evidence (SOE) for key outcomes according to methods as described in the AHRQ Methods Guide. ${ }^{21}$ The overall strength of evidence was determined based on assessment of study limitations (or ROB, graded low, moderate, or high); consistency of results across trials (graded consistent, inconsistent, or for single studies, unknown); the directness of the evidence linking the interventions with clinical outcomes (graded direct or indirect); effect estimate precision (graded precise or imprecise); and reporting bias (suspected or undetected). Based on these assessments, SOE was appraised as high, moderate, low, or insufficient to estimate an effect (See Appendix B for more information on Grading the SOE). 
Plain-language statements are used in the main points of the Evidence Summary and key points of the Results to convey the SOE: High SOE is described as "is associated with" or simply "reduces/increases;" moderate SOE is described as "likely" or "probably;" and low SOE is described as "may be" or "might be." To ensure consistency and validity of assessments, the strength of evidence grade was reviewed by the entire team of investigators prior to assigning a final grade.

Bodies of evidence consisting of RCTs were initially considered high SOE and downgraded depending on study limitations. In contrast, bodies of evidence consisting of observational studies were initially considered low SOE. Observational studies pose a greater risk of having study limitations because of the typically higher risk of bias due to lack of randomization. However, per AHRQ Methods Guide, EPCs can move up the initial SOE grade for observational studies to moderate if study ROB was rated low or moderate and the study authors performed analyses to control for risk of bias. For reviews that addressed KQ 1 and included cohort trials, we rated the initial SOE as moderate if the authors of the reviews rated the ROB of included studies as low or moderate and indicated that studies conducted analyses to control for critical confounders, such as age and severity of illness.

To assess SOE of findings from existing SRs, if reviews utilized a grading system similar to that described in AHRQ's methods guide, we used the SOE ratings reported by review authors. If reviews used a compatible grading system (e.g., GRADE system), we translated the review's evidence ratings to AHRQ's SOE ratings. If the review did not assess the overall quality of the evidence, we assessed it ourselves, using AHRQ's grading system (provided evidence was reported in a manner that allowed us to judge the overall risk of bias, consistency, directness, and precision of evidence). However, if reviews reported insufficient information to allow for accurate appraisal, we did not attempt to rate the SOE.

\section{Applicability}

We followed procedures outlined in the AHRQ Methods Guide to assess the applicability of the findings within and across studies. ${ }^{21}$ Applicability for each outcome was summarized and presented qualitatively using the PICOTS framework and not a specific checklist or scale. Several a priori patient factors may limit the applicability of findings, including age, gender, comorbidities, and illness severity. The following population relevant measures are frequently used within hospital settings to measure severity of illness: Sequential Organ Failure Assessment (SOFA score), Simplified Acute Physiology Score (SAPSII), and Acute Physiology and Chronic Health Evaluation (APACHE).

Small sample size may be an important limitation in many studies and addressing this through meta-analysis may be challenging if there is substantial heterogeneity in study design, intervention, and outcome reporting. Confounding factors such as the severity of illness that studies are unable to or fail to control may impact the relationship between malnutrition and clinical outcomes. We reported any limitations in applicability of individual studies within evidence tables and limitations of applicability of the whole body of evidence in the summary of evidence tables. 


\section{Results}

\section{Search Results}

Electronic searches identified 3,308 citations. After title and abstract screening, 83 required full text review and 17 studies met eligibility criteria for inclusion in this review (Figure 3). A list of the studies excluded at the full-text review stage is in Appendix C. The primary reasons for exclusion were wrong patient population (e.g., patients not hospitalized or did not meet criteria for malnutrition for Key Question [KQ] 3), not an appropriate study design (e.g., retrospective or did not include appropriate care pathway for KQ 2), and wrong setting (e.g., took place outside of eligible countries). 
Figure 3. Study flow diagram

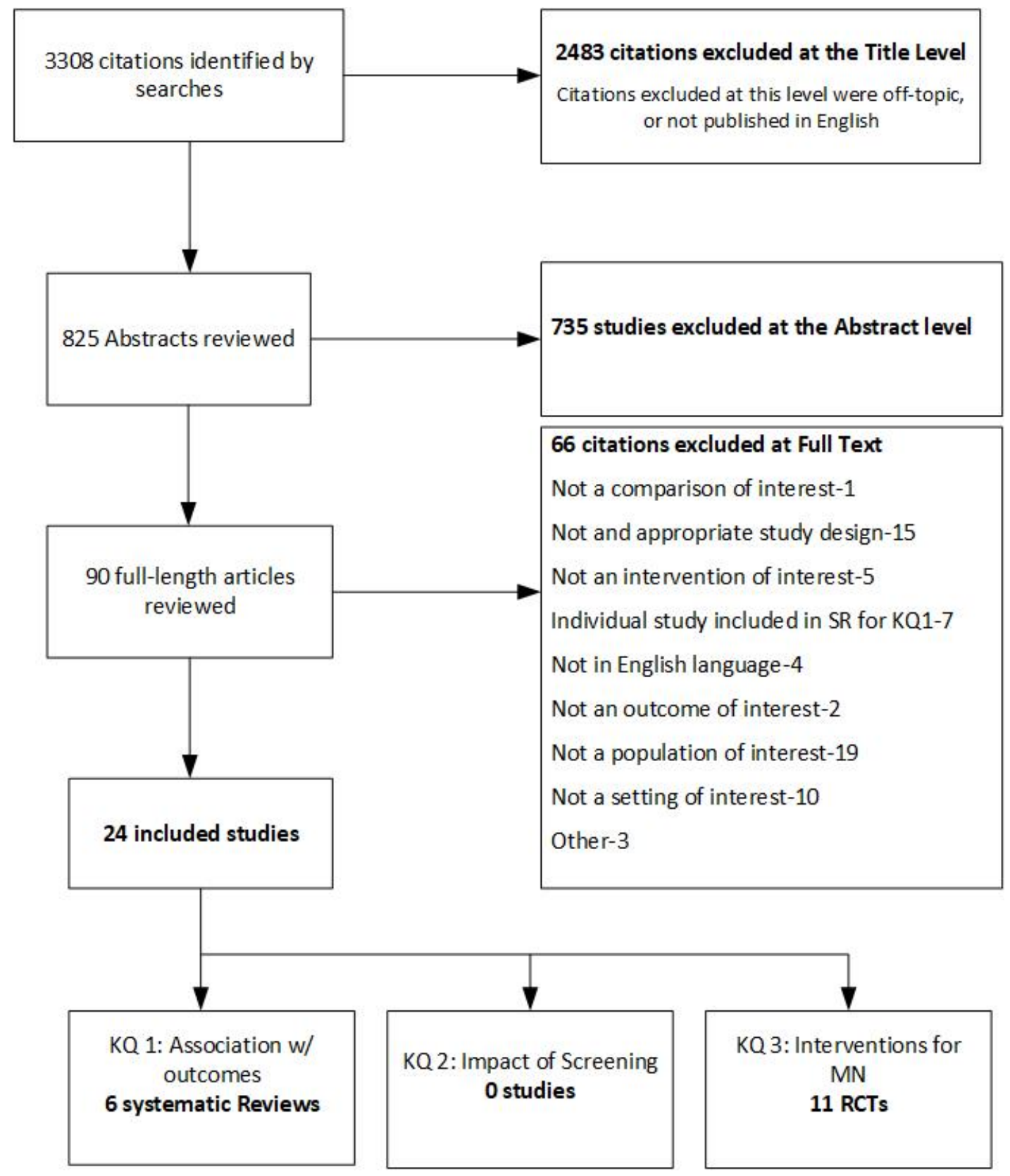

$. \mathrm{KQ}=$ Key Question; $\mathrm{MN}=$ malnutrition; $\mathrm{RCTs}=$ randomized controlled trials; $\mathrm{SRs}=$ systematic reviews

Below we provide the report results, including the Key Points for each Key Question, and describe the included evidence, as well as the data synthesis and a summary of the strength of evidence. Details on results of literature searches, included studies, and the strength of evidence can be found in Appendix F. 
Key Question 1. What is the association between malnutrition and clinical outcomes among hospitalized patients?
a. How do outcomes vary depending on measures or tools used to detect malnutrition?
b. Are patient-related risk factors, such as increased age or certain pre- existing health conditions, associated with poorer clinical outcomes?

\section{Description of Included Evidence}

Our searches identified 6 systematic reviews (SRs) (including 43 relevant unique studies) evaluating the association between malnutrition and clinical outcomes among hospitalized patients. The patient population, tools used to assess malnutrition, and outcomes reported varied across SRs and, in some cases, among studies included in the reviews. Key characteristics of the SRs and their included studies are summarized in Table 3. Detailed information about the reviews and included studies is provided in Appendix D (Table D-1 to Table D-2). Evidence from some studies included in these reviews was not considered in this report as these studies did not meet inclusion criteria. For instance, we did not consider evidence from retrospective studies (except when combined with prospective studies in a meta-analysis), studies of non-hospitalized patients, or studies that did not use commonly available measurement tools to assess malnutrition.

Most studies included in the SRs were prospective cohort trials in which patients were hospitalized for a range of conditions, including traumatic injury, ${ }^{30}$ acute medical conditions requiring care in the intensive care unit (e.g., pneumonia, acute kidney failure), ${ }^{31}$ heart failure, ${ }^{32}$ cancer ${ }^{33}$ chronic obstructive pulmonary disease (COPD),${ }^{34}$ and liver transplant. ${ }^{35}$ Malnutrition prevalence varied across the included studies, ranging from 7 percent to 90 percent depending on patient population and tool used to diagnose malnutrition. Many studies used screening tools, such as the Nutritional Risk Screening-2002 (NRS-2002) or the Malnutrition Universal Screening Tool (MUST), to categorize patients with malnutrition.

Using the Risk of Bias in Systematic Reviews (ROBIS) instrument, we rated the risk of bias (ROB) of 5 of the 6 included SRs as Low (see Table E-1 in Appendix E for ratings). Study eligibility, data collection, study appraisal, and synthesis of findings of these SRs were clearly described and appropriate. One SR did not use a formal tool to assess the ROB of included studies but provided sufficient information about study design and methods to allow us to appraise individual study ROB. The ROB of the studies included in the SRs ranged from low to high, with review authors rating most of the studies within the moderate range. 
Table 3. Key characteristics of included systematic reviews

\begin{tabular}{|c|c|c|c|c|c|c|}
\hline Reference & $\begin{array}{l}\text { Total Studies } \\
\text { Included in SR }\end{array}$ & $\begin{array}{l}\text { Number of Relevant } \\
\text { Studies Reporting } \\
\text { Outcomes of Interest }\end{array}$ & $\begin{array}{l}\text { Patient Populations } \\
\% \text { With Malnutrition }\end{array}$ & $\begin{array}{l}\text { Screening/ Diagnostic } \\
\text { Tools of Interest for } \\
\text { Malnutrition* }\end{array}$ & $\begin{array}{l}\text { Outcomes } \\
\text { Reported }\end{array}$ & $\begin{array}{l}\text { Type of } \\
\text { Synthesis }\end{array}$ \\
\hline $\begin{array}{l}\text { Dijkink et al. } \\
2020^{30}\end{array}$ & $\begin{array}{l}13 \text { cohort trials } \\
\text { (11 prospective, } \\
2 \text { retrospective) }\end{array}$ & 8 & $\begin{array}{l}\text { Traumatic injury } \\
7 \text { to } 76 \%, d x \text { based on } \\
\text { SGA or MNA in } 6 \text { studies }\end{array}$ & $\begin{array}{l}\text { Screening tools: } \\
\text { NRS-2002: } 2 \text { studies } \\
\text { Diagnostic tools: } \\
\text { MNA: } 4 \text { studies } \\
\text { SGA: } 2 \text { studies }\end{array}$ & $\begin{array}{l}\text { Mortality, length } \\
\text { of stay, 30-day } \\
\text { readmission, } \\
\text { HAC }\end{array}$ & Qualitative \\
\hline $\begin{array}{l}\text { Ney et al. } \\
2019^{35}\end{array}$ & $\begin{array}{l}47 \text { cohort trials (type } \\
\text { of cohort trial not } \\
\text { reported) }\end{array}$ & 3 & $\begin{array}{l}\text { Cirrhosis } \\
8.0 \% \text { to } 100 \% \text { based on } \\
\text { SGA }\end{array}$ & $\begin{array}{l}\text { Screening tools: no studies } \\
\text { Diagnostic tools: } \\
\text { SGA: } 3 \text { studies }\end{array}$ & $\begin{array}{l}\text { Pre-and Post- } \\
\text { mortality, ICU } \\
\text { length of stay }\end{array}$ & $\begin{array}{l}\text { Quantitative } \\
\text { meta-analysis }\end{array}$ \\
\hline $\begin{array}{l}\text { Muscariotoli } \\
\text { et al. } 2018^{34}\end{array}$ & $\begin{array}{l}15 \text { studies } \\
\text { (12 prospective } \\
\text { cohort studies and } \\
3 \text { database studies) }\end{array}$ & 7 & $\begin{array}{l}\text { Various conditions, } \\
\text { including general medicine, } \\
\text { COPD, heart failure, and } \\
\text { pneumonia } \\
\text { NR }\end{array}$ & $\begin{array}{l}\text { Screening tools: } \\
\text { NRI: } 1 \text { study } \\
\text { MUST: } 1 \text { study } \\
\text { Diagnostic tools: } \\
\text { SGA: } 3 \text { studies } \\
\text { MNA: } 2 \text { studies }\end{array}$ & $\begin{array}{l}\text { 30-day } \\
\text { readmission }\end{array}$ & Qualitative \\
\hline $\begin{array}{l}\text { Lew et al. } \\
2016^{31}\end{array}$ & $\begin{array}{l}20 \text { prospective } \\
\text { cohort trials } \\
\text { (15 were used to } \\
\text { assess outcomes) }\end{array}$ & 6 & $\begin{array}{l}\text { Acute medical conditions } \\
\text { requiring ICU care (e.g., } \\
\text { acute kidney failure) } \\
37.8 \text { to } 78.1 \% \text {, all dx with } \\
\text { SGA or MNA }\end{array}$ & $\begin{array}{l}\text { Screening tools: } \\
\text { NRS-2002: } 4 \text { studies } \\
\text { MUST: } 3 \text { studies } \\
\text { MNA-SF: } 1 \text { study } \\
\text { PINI: } 1 \text { study } \\
\text { Diagnostic tools: } \\
\text { SGA: } 10 \text { studies } \\
\text { MNA: } 2 \text { studies }\end{array}$ & $\begin{array}{l}\text { Mortality, length } \\
\text { of stay, 30-day } \\
\text { readmission, } \\
\text { HAC, wound } \\
\text { healing, } \\
\text { discharge } \\
\text { disposition }\end{array}$ & Qualitative \\
\hline
\end{tabular}




\begin{tabular}{|c|c|c|c|c|c|c|}
\hline Reference & $\begin{array}{l}\text { Total Studies } \\
\text { Included in SR }\end{array}$ & $\begin{array}{l}\text { Number of Relevant } \\
\text { Studies Reporting } \\
\text { Outcomes of Interest }\end{array}$ & $\begin{array}{l}\text { Patient Populations } \\
\% \text { With Malnutrition }\end{array}$ & $\begin{array}{l}\text { Screening/ Diagnostic } \\
\text { Tools of Interest for } \\
\text { Malnutrition }^{*}\end{array}$ & $\begin{array}{l}\text { Outcomes } \\
\text { Reported }\end{array}$ & $\begin{array}{l}\text { Type of } \\
\text { Synthesis }\end{array}$ \\
\hline $\begin{array}{l}\text { Lin et al. } \\
2016^{32}\end{array}$ & $\begin{array}{l}17 \text { trials } \\
(12 \text { prospective } \\
\text { cohort, } \\
5 \text { retrospective })\end{array}$ & 11 & $\begin{array}{l}\text { Heart failure } \\
\text { General: } 16 \text { to } 90 \% \\
\text { By tool: } \\
\text { MNA: } 16 \text { to } 90 \% \\
\text { GNRI: } 22 \text { to } 48 \% \\
\text { NRI: } 23 \text { to } 90 \% \\
\text { NRS: } 57.3 \%\end{array}$ & $\begin{array}{l}\text { Screening tools: } \\
\text { GNRI: } 4 \text { studies } \\
\text { NRI: } 3 \text { studies } \\
\text { MNA-SF: } 2 \text { studies } \\
\text { SCORE: } 1 \text { study } \\
\text { NRS-2002: } 1 \text { study } \\
\text { CONUT: } 1 \text { study } \\
\text { PNI: } 1 \text { study } \\
\text { Other: } 2 \text { studies } \\
\text { Diagnostic tools: } \\
\text { MNA: } 5 \text { studies }\end{array}$ & $\begin{array}{l}\text { Mortality, length } \\
\text { of stay, HAC }\end{array}$ & $\begin{array}{l}\text { Quantitative } \\
\text { meta-analysis } \\
\text { of mortality, } \\
\text { other outcomes } \\
\text { qualitative }\end{array}$ \\
\hline $\begin{array}{l}\text { Gupta et al. } \\
2011^{33}\end{array}$ & $\begin{array}{l}8 \text { cohort trials, } \\
(6 \text { prospective, } \\
2 \text { retrospective })\end{array}$ & 8 & $\begin{array}{l}\text { Cancer } \\
\text { Moderate to severe MN: } \\
42 \%\end{array}$ & $\begin{array}{l}\text { Screening tools: no studies } \\
\text { Diagnostic tools: } \\
\text { SGA: } 6 \text { studies } \\
\text { PG-SGA: } 2 \text { studies }\end{array}$ & Length of stay & Qualitative \\
\hline
\end{tabular}

Studies that did not use a known tool to measure nutritional status were not included in this column.

CONUT $=$ Controlling Nutritional Status Score; GNRI = Geriatric Nutritional Risk Index; HAC = hospital acquired condition; MNA = Mini Nutritional Assessment; MNA-SF = MNA-short form; MUST = Malnutrition Universal Screening Tool; NR = not reported; NRI = Nutritional Risk Index; NRS-2002 = Nutritional Risk Score, PINI = Prognostic Inflammatory and Nutrition Index; SGA = Subjective Global Assessment; SNAQ = Short Nutritional Assessment Questionnaire 


\section{Key Points}

\section{Patients Hospitalized for Traumatic Injury}

- Patients hospitalized due to traumatic injury and screened at risk of malnutrition (using NRS-2002) may experience more hospital acquired conditions compared to wellnourished patients hospitalized for a traumatic injury. (strength of evidence [SOE]: Low)

- Evidence for other outcomes and measurement tools used to identify malnutrition (Subjective Global Assessment [SGA]) was insufficient to determine if malnutrition was associated with clinical outcomes among patients hospitalized for a traumatic injury.

\section{Patients Hospitalized for an Acute Medical Condition Requiring Intensive Care Unit Care}

- Patients requiring intensive care unit (ICU) care and diagnosed with malnutrition (using Subjective Global Assessment [SGA]) may have higher hospital mortality compared to well-nourished patients requiring ICU care.

(SOE: Low)

- Patients requiring ICU care and diagnosed with malnutrition (using SGA) are likely to experience prolonged hospital length of stay compared to well-nourished patients requiring ICU care. (SOE: Moderate)

- Patients requiring ICU care and diagnosed with malnutrition (using Mini Nutritional Assessment [MNA]) may experience more hospital acquired conditions compared to well-nourished patients requiring ICU care. (SOE: Low) No other differences were observed using MNA to assess nutritional status.

- Evidence for other measurement tools used to identify malnutrition (Nutrition Risk Screening-2002, Malnutrition Universal Screening Tool, Mini Nutritional AssessmentShort Form, Short Nutritional Assessment Questionnaire, and Prognosis Inflammatory and Nutritional Index) was insufficient to determine if malnutrition was associated with clinical outcomes among patients requiring ICU care.

\section{Patients Hospitalized for Decompensations of Chronic Disease}

- Patients hospitalized with heart failure and diagnosed with malnutrition (using several different measurement tools) may have higher mortality compared to well-nourished patients with heart failure. (SOE: Low)

- Patients hospitalized with cancer and diagnosed with malnutrition (using SGA) may experience prolonged hospital length of stay compared to well-nourished patients (SOE: Low)

- Patients hospitalized with cirrhosis awaiting transplantation and diagnosed with malnutrition (using SGA) may have higher pre-transplant mortality compared to wellnourished patients. (SOE: Low) 


\section{Summary of Findings}

\section{Malnutrition Among Patients Hospitalized for Traumatic Injury or Acute Medical Conditions Requiring ICU Care}

In traumatically injured patients, the relationship between nutritional status and clinical outcomes is complicated by the systemic pathophysiological responses to trauma, which may affect, as well as be affected by the patient's nutritional status. ${ }^{30}$ Thus, the impact of nutritional status on clinical outcomes of patients who have experienced a traumatic injury or an acute medical event can be difficult to interpret.

Our literature search identified 2 SRs (including 14 relevant studies) that provided evidence on the association between malnutrition and clinical outcomes among patients hospitalized for a traumatic injury ${ }^{30}$ or receiving ICU care for acute complications related to an existing medical condition or surgical procedure. ${ }^{30,31}$ The SR by Lew et al. (2016) only considered findings from studies rated as low risk of bias ( 6 out of 14 included). ${ }^{31}$ The median prevalence of malnutrition on admission across both SRs using the SGA or MNA was 37.9 percent, range 7 percent to 78 percent. The prevalence of malnutrition varied depending on the patient population, with older patients, patients in the ICU, and patients with acute kidney disease having higher rates of malnutrition. Table 4 presents the SOE ratings for all assessed outcomes by measurement tool. The table is organized by measurement tool and outcome and includes columns indicating what study provided the data (reference), findings and direction of findings (e.g., no association, increased/decreased occurrence of outcome), and strength of evidence rating.

\section{Subjective Global Assessment}

Four studies included in the SR by Lew et al. (2016) enrolling a total of 729 patients evaluated the association between malnutrition (diagnosed using SGA) and mortality among patients with acute medical conditions receiving care in the ICU. ${ }^{31}$ Two studies demonstrated that patients requiring ICU care and diagnosed with malnutrition (MN) may be at increased risk of mortality compared to well-nourished (WN) patients. One study showed that patients with malnutrition were 8 times as likely as well-nourished patients to experience mortality (Fontes, $2014, \mathrm{n}=185$, adjusted odds ratio [AOR]: $8.12,95$ percent confidence interval [CI]: 2.94 to $22.42, \mathrm{p}<0.05$ ). The findings of the other study (Sheean et al 2013) enrolling 260 patients reported a mortality rate of 23 percent among patients diagnosed with malnutrition compared to 4.8 percent among well-nourished patients $(\mathrm{p}<0.01)$. Two other studies reported a non-significant trend toward an association between malnutrition and increased mortality.

One study from the SR by Dijkink et al (2020) enrolling 161 assessed the association between malnutrition (diagnosed using SGA) and mortality among patients admitted to the hospital for a traumatic injury. ${ }^{30,31}$ However, the SOE for this outcome was rated insufficient as the sample size was small and the width of the confidence intervals surrounding the summary estimate suggested some uncertainty (Goiburu, 2006, $n=161$, relative risk [RR]: 4.0, 95 percent CI: 1.0 to 15.0). Table 5 presents the SOE ratings for all outcomes by measurement tool.

Four other studies ( $\mathrm{n}=1,737$, Caporossi, 2012; Merli, 2010; Lomivorotov, 2013; Sheean, $2013)^{31}$ found that patients requiring ICU care and diagnosed with malnutrition (using SGA) were more likely to experience prolonged hospital length of stay compared to well-nourished patients (SOE: Moderate). Evidence on the association between malnutrition (diagnosed using SGA) and other clinical outcomes - 30-day readmission, discharge disposition, and hospital acquired complications_-was insufficient. 


\section{Mini Nutritional Assessment}

The SRs included 3 studies using the MNA to measure the association of malnutrition with clinical outcomes. ${ }^{30,31}$ However, these studies did not find any association between malnutrition and increased mortality or length of stay (Goisser, 2015, $n=97$; Lomivorotov, 2013, $n=1193$; Sheean, 2013, n=260). One study found that patients requiring ICU care and diagnosed with malnutrition (using MNA) may experience more hospital acquired complications compared to well-nourished patients requiring ICU care (Lomivorotov, 2013, n=1193, AOR: 1.60, 95percent CI: 1.10 to 2.20 , SOE: Low)

\section{Other Tools}

Other studies included in the 2 SRs evaluated malnutrition using one of the following screening tools - Nutrition Risk Screening-2002 (NRS-2002), Malnutrition Universal Screening Tool (MUST), Mini Nutritional Assessment-Short Form, Short Nutritional Assessment Questionnaire, and Prognosis Inflammatory and Nutritional Index. Tools, such as the NRS-2002 and MUST, showed either no or inconsistent associations between malnutrition and poor clinical outcomes. 
Table 4. Strength of evidence ratings by measurement tool for trauma injury/ICU patients

\begin{tabular}{|c|c|c|c|c|c|c|}
\hline Intervention & Outcome & Population & Reference & $\begin{array}{l}\text { Number and } \\
\text { Type of Study } \\
\text { (\# Patients) }\end{array}$ & Direction of Association/Findings & Overall SoE/Domains \\
\hline SGA & Mortality & $\begin{array}{l}\text { Traumatic } \\
\text { Injury }\end{array}$ & $\begin{array}{l}\text { 1 SR, Dijkink } \\
\text { et al. } 2020^{30}\end{array}$ & $\begin{array}{l}1 \text { prospective } \\
\text { cohort trial } \\
\text { (161) }\end{array}$ & $\begin{array}{l}\text { Inconclusive } \\
\text { Goiburu et al. } 2006, n=161, \text { RR: } 4.0 \text {, } \\
95 \% \mathrm{Cl}: 1.0 \text { to } 15.0\end{array}$ & $\begin{array}{l}\text { Insufficient } \\
\text { Risk of bias: High } \\
\text { Consistency: Unknown } \\
\text { Directness: Direct } \\
\text { Precision: Imprecise (small sample size) }\end{array}$ \\
\hline SGA & Mortality & ICU & $\begin{array}{l}\text { 1 SR Lew } \\
\text { et al. } 2016^{31}\end{array}$ & $\begin{array}{l}4 \text { prospective } \\
\text { cohort trials } \\
(729)\end{array}$ & $\begin{array}{l}\text { Increased } \\
\text { Fontes et al. } 2014, n=185, \text { AOR: } 8.12, \\
95 \% \text { Cl: } 2.94 \text { to } 22.42, p<0.05 ; \text { Sheean } \\
\text { et al. 2013, } n=260, W N: 4.8 \%, M N \text { : } \\
23.0 \%, p<0.01 \\
2 \text { studies non-significant trend for } \\
\text { increase (Capossi et al. 2012, } n=246, \\
\text { AOR: } 2.00,95 \% \text { Cl: } 0.50 \text { to } 7.60 ; \text { Merli } \\
\text { et al. } 2010, n=38, p=0.10 \text { ) }\end{array}$ & $\begin{array}{l}\text { Low } \\
\text { Risk of bias: Moderate } \\
\text { Consistency: Consistent } \\
\text { Directness: Direct } \\
\text { Precision: Imprecise (small sample size) }\end{array}$ \\
\hline SGA & \begin{tabular}{|l|} 
Length of Stay \\
${ }^{*}$ Ratio statistics \\
measure \\
likelihood of \\
staying longer
\end{tabular} & $\begin{array}{l}\text { Traumatic } \\
\text { Injury }\end{array}$ & $\begin{array}{l}\text { 1 SR, Dijkink } \\
\text { et al. } 2020^{30}\end{array}$ & $\begin{array}{l}1 \text { prospective } \\
\text { cohort trial } \\
\text { (161) }\end{array}$ & $\begin{array}{l}\text { Inconclusive } \\
\text { Goiburu et al. } 2006, \mathrm{n}=161: \text { Hosp } \\
>14 \text { days, RR: } 2.30,95 \% \mathrm{Cl}: 1.2 \text { to } 4.7\end{array}$ & $\begin{array}{l}\text { Insufficient } \\
\text { Risk of bias: High } \\
\text { Consistency: Unknown } \\
\text { Directness: Direct } \\
\text { Precision: Imprecise (small sample size) }\end{array}$ \\
\hline SGA & $\begin{array}{l}\text { Length of Stay } \\
\text { *Ratio statistics } \\
\text { measure } \\
\text { likelihood of } \\
\text { staying longer }\end{array}$ & ICU & $\begin{array}{l}\text { 1 SR Lew } \\
\text { et al. } 2016^{31}\end{array}$ & $\begin{array}{l}4 \text { prospective } \\
\text { cohort trials } \\
(1,737)\end{array}$ & 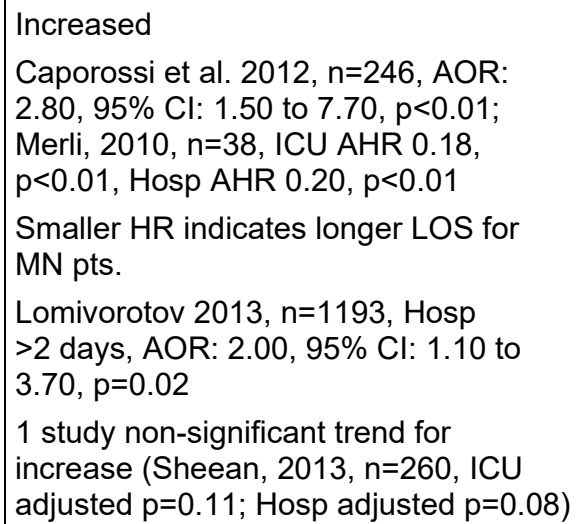 & $\begin{array}{l}\text { Moderate } \\
\text { Risk of bias: Moderate } \\
\text { Consistency: Consistent } \\
\text { Directness: Direct } \\
\text { Precision: Precise }\end{array}$ \\
\hline
\end{tabular}




\begin{tabular}{|c|c|c|c|c|c|c|}
\hline Intervention & Outcome & Population & Reference & $\begin{array}{l}\text { Number and } \\
\text { Type of Study } \\
\text { (\# Patients) }\end{array}$ & Direction of Association/Findings & Overall SoE/Domains \\
\hline SGA & $\begin{array}{l}\text { ICU } \\
\text { readmission } \\
\text { during index } \\
\text { hospital stay }\end{array}$ & ICU & $\begin{array}{l}1 \text { SR, Lew } \\
\text { et al. } 2016^{31}\end{array}$ & $\begin{array}{l}1 \text { prospective } \\
\text { cohort trial } \\
(185)\end{array}$ & $\begin{array}{l}\text { Inconclusive } \\
\text { Fontes et al. } 2014, \text { AOR: } 2.27,95 \% \mathrm{Cl} \text { : } \\
1.08 \text { to } 4.80, \mathrm{p}<0.005\end{array}$ & $\begin{array}{l}\text { Insufficient } \\
\text { Risk of bias: Moderate } \\
\text { Consistency: Unknown } \\
\text { Directness: Direct } \\
\text { Precision: Imprecise (small sample size) }\end{array}$ \\
\hline SGA & $\begin{array}{l}\text { HAC (delayed } \\
\text { wound healing) }\end{array}$ & ICU & $\begin{array}{l}1 \text { SR, Lew } \\
\text { et al. } 2016^{31}\end{array}$ & $\begin{array}{l}1 \text { prospective } \\
\text { cohort trial (38) }\end{array}$ & $\begin{array}{l}\text { Inconclusive } \\
\text { Merli et al. 2010, higher incidence of } \\
\text { infection associated with MN compared } \\
\text { to WN ( } 4.5 \text { vs } 0.6 \text { episodes per patient, } \\
\text { adjusted, } p<0.001)\end{array}$ & $\begin{array}{l}\text { Insufficient } \\
\text { Risk of bias: Moderate } \\
\text { Consistency: Unknown } \\
\text { Directness: Direct } \\
\text { Precision: Imprecise (small sample size) }\end{array}$ \\
\hline SGA & $\begin{array}{l}\text { Discharge } \\
\text { Disposition }\end{array}$ & ICU & $\begin{array}{l}1 \text { SR, Lew } \\
\text { et al. } 2016^{31}\end{array}$ & $\begin{array}{l}1 \text { prospective } \\
\text { cohort trial } \\
(260)\end{array}$ & $\begin{array}{l}\text { Inconclusive } \\
\text { Sheean et al. 2013, 28.6\% fewer MN } \\
\text { pts discharged to home than well- } \\
\text { nourished pts; adjusted } p<0.001\end{array}$ & $\begin{array}{l}\text { Insufficient } \\
\text { Risk of bias: Moderate } \\
\text { Consistency: Unknown } \\
\text { Directness: Direct } \\
\text { Precision: Imprecise (small sample size) }\end{array}$ \\
\hline MNA & Mortality & ICU & $\begin{array}{l}1 \text { SR, Lew } \\
\text { et al. } 2016^{31}\end{array}$ & $\begin{array}{l}2 \text { prospective } \\
\text { cohort trials } \\
(1,453)\end{array}$ & $\begin{array}{l}\text { No association } \\
\text { Lomivorotov et al. 2013, } n=1193, \\
p>0.05 ; \text { Sheean et al. } 2013, n=260 \text {, adj } \\
p=0.09\end{array}$ & $\begin{array}{l}\text { Low } \\
\text { Risk of bias: Moderate } \\
\text { Consistency: Consistent } \\
\text { Directness: Direct } \\
\text { Precision: Imprecise (due to } p>0.05 \text { ) }\end{array}$ \\
\hline MNA & Length of Stay & ICU & $\begin{array}{l}1 \mathrm{SR}, \mathrm{Lew} \\
\text { et al. } 2016^{31}\end{array}$ & $\begin{array}{l}2 \text { prospective } \\
\text { cohort trials } \\
(1,752)\end{array}$ & $\begin{array}{l}\text { No association } \\
\text { Lomivorotov et al } 2013, \mathrm{n}=1193,>2 \text {-day } \\
\text { ICU stay, AOR: } 1.40,95 \% \mathrm{Cl}: 0.70 \text { to } \\
\text { 2.30; Sheean et al. } 2013, \mathrm{n}=260 \text {, adj } \\
p=0.17 \text { Hosp, } p=0.07 \text { ICU }\end{array}$ & $\begin{array}{l}\text { Low } \\
\text { Risk of bias: Moderate } \\
\text { Consistency: Consistent } \\
\text { Directness: Direct } \\
\text { Precision: Imprecise (due to } p>0.05 \text { ) }\end{array}$ \\
\hline
\end{tabular}




\begin{tabular}{|c|c|c|c|c|c|c|}
\hline Intervention & Outcome & Population & Reference & $\begin{array}{l}\text { Number and } \\
\text { Type of Study } \\
\text { (\# Patients) }\end{array}$ & Direction of Association/Findings & Overall SoE/Domains \\
\hline MNA & $\begin{array}{l}\text { HAC (post- } \\
\text { operation } \\
\text { complication) }\end{array}$ & ICU & $\begin{array}{l}1 \text { SR, Lew } \\
\text { et al. } 2016^{31}\end{array}$ & $\begin{array}{l}1 \text { prospective } \\
\text { cohort trial } \\
(1,193)\end{array}$ & $\begin{array}{l}\text { Increased } \\
\text { Lomivorotov et al. 2013, Post-op } \\
\text { complications, AOR: } 1.60,95 \% \mathrm{Cl}: 1.10 \\
\text { to } 2.20, \mathrm{p}<0.01\end{array}$ & $\begin{array}{l}\text { Low } \\
\text { Risk of bias: Moderate } \\
\text { Consistency: Unknown } \\
\text { Directness: Direct } \\
\text { Precision: Precise (single study) }\end{array}$ \\
\hline NRS-2002 & Mortality & ICU & $\begin{array}{l}1 \text { SR, Lew } \\
\text { et al. } 2016^{31}\end{array}$ & $\begin{array}{l}1 \text { prospective } \\
\text { cohort trial } \\
(260)\end{array}$ & $\begin{array}{l}\text { Inconclusive } \\
1 \text { study found MN associated with } \\
\text { greater hospital mortality (Sheean et al. } \\
2013 \text {, adjusted } p=0.03 \text { ) compared to } \\
\text { WN }\end{array}$ & $\begin{array}{l}\text { Insufficient } \\
\text { Risk of bias: Moderate } \\
\text { Consistency: Unknown } \\
\text { Directness: Direct } \\
\text { Precision: Imprecise (small sample size) }\end{array}$ \\
\hline NRS-2002 & Length of Stay & ICU & $\begin{array}{l}1 \text { SR, Lew } \\
\text { et al. } 2016^{31}\end{array}$ & $\begin{array}{l}2 \text { prospective } \\
\text { cohort trials } \\
(1,453)\end{array}$ & $\begin{array}{l}\text { Inconclusive } \\
1 \text { of } 2 \text { studies found MN associated with } \\
\text { increased LOS }>2 \text { days (Lomivorotov } \\
\text { et al. } 2013, n=1193, \text { AOR: } 1.80 \text {, } \\
95 \% \mathrm{Cl}: 1.10 \text { to } 3.30 \text { ) } \\
\text { No difference: Sheean et al. } 2013 \text {, } \\
n=260\end{array}$ & $\begin{array}{l}\text { Insufficient } \\
\text { Risk of bias: Moderate } \\
\text { Consistency: Inconsistent } \\
\text { Directness: Direct } \\
\text { Precision: Imprecise }\end{array}$ \\
\hline NRS-2002 & HAC & $\begin{array}{l}\text { Traumatic } \\
\text { injury }\end{array}$ & $\begin{array}{l}1 \text { SR, Dijkink } \\
\text { et al. } 2020^{30}\end{array}$ & $\begin{array}{l}2 \text { prospective } \\
\text { cohort trials } \\
(2,163)\end{array}$ & $\begin{array}{l}\text { Increased } \\
\text { Ihle et al. } 2017, n=521: \geq 1 \mathrm{AE} \\
\text { associated with } \mathrm{MN}(\mathrm{p}<0.001) \\
\text { Wintermeyer et al. } 2019, \mathrm{n}=1,642 \text { : } \\
\geq 1 \mathrm{AE} \text { associated with } \mathrm{MN}(\mathrm{p}<0.001)\end{array}$ & $\begin{array}{l}\text { Low } \\
\text { Risk of bias: High } \\
\text { Consistency: Consistent } \\
\text { Directness: Direct } \\
\text { Precision: Precise }\end{array}$ \\
\hline MUST & Mortality & ICU & $\begin{array}{l}1 \text { SR, Lew } \\
\text { et al. } 2016^{31}\end{array}$ & $\begin{array}{l}1 \text { prospective } \\
\text { cohort (109) }\end{array}$ & $\begin{array}{l}\text { Inconclusive } \\
\text { Tripathy et al. } 2014 \text {, found MN } \\
\text { associated with 1-year mortality (AOR: } \\
2.94,95 \% \mathrm{Cl}: 1.10 \text { to } 8.00 \text { ) }\end{array}$ & $\begin{array}{l}\text { Insufficient } \\
\text { Risk of bias: Moderate } \\
\text { Consistency: Unknown } \\
\text { Directness: Direct } \\
\text { Precision: Imprecise (small sample size) }\end{array}$ \\
\hline
\end{tabular}




\begin{tabular}{|c|c|c|c|c|c|c|}
\hline Intervention & Outcome & Population & Reference & $\begin{array}{l}\text { Number and } \\
\text { Type of Study } \\
\text { (\# Patients) }\end{array}$ & Direction of Association/Findings & Overall SoE/Domains \\
\hline MUST & Length of stay & ICU & $\begin{array}{l}1 \text { SR, Lew } \\
\text { et al. } 2016^{31}\end{array}$ & $\begin{array}{l}1 \text { prospective } \\
\text { cohort }(1,193)\end{array}$ & $\begin{array}{l}\text { Inconclusive } \\
\text { Lomivorotov et al. } 2013 \text {, found no } \\
\text { association, AOR }>2 \text { day of ICU stay: } \\
1.20,95 \% \mathrm{Cl}: 0.90 \text { to } 2.00, p=0.33\end{array}$ & $\begin{array}{l}\text { Insufficient } \\
\text { Risk of bias: Moderate } \\
\text { Consistency: Unknown } \\
\text { Directness: Direct } \\
\text { Imprecision: Imprecise }(p>0.05)\end{array}$ \\
\hline MUST & HAC & ICU & $\begin{array}{l}1 \text { SR, Lew } \\
\text { et al. } 2016^{31}\end{array}$ & $\begin{array}{l}1 \text { prospective } \\
\text { cohort }(1,193)\end{array}$ & $\begin{array}{l}\text { Inconclusive } \\
\text { Lomivorotov et al. } 2013 \text {, found no } \\
\text { association with post-operative } \\
\text { complications, AOR: } 1.3,95 \% \mathrm{Cl}: 0.90 \\
\text { to } 2.00), p=0.11\end{array}$ & $\begin{array}{l}\text { Insufficient } \\
\text { Risk of bias: Moderate } \\
\text { Consistency: Unknown } \\
\text { Directness: Direct } \\
\text { Imprecision: Imprecise }(p>0.05)\end{array}$ \\
\hline MNA-SF & Mortality & ICU & $\begin{array}{l}1 \text { SR, Lew } \\
\text { et al. } 2016^{31}\end{array}$ & $\begin{array}{l}1 \text { prospective } \\
\text { cohort (260) }\end{array}$ & $\begin{array}{l}\text { Inconclusive } \\
\text { Sheean et al. 2013, adj } p<0.01 \\
\text { (no further details reported) }\end{array}$ & $\begin{array}{l}\text { Insufficient } \\
\text { Risk of bias: Moderate } \\
\text { Consistency: Unknown } \\
\text { Directness: Direct } \\
\text { Precision: Imprecise (small sample size) }\end{array}$ \\
\hline MNA-SF & Length of Stay & ICU & $\begin{array}{l}1 \text { SR, Lew } \\
\text { et al. } 2016^{31}\end{array}$ & $\begin{array}{l}1 \text { prospective } \\
\text { cohort }(260)\end{array}$ & $\begin{array}{l}\text { Inconclusive } \\
\text { Sheean et al. 2013, adj } p=0.06 \\
\text { (no further details reported) }\end{array}$ & $\begin{array}{l}\text { Insufficient } \\
\text { Risk of bias: Moderate } \\
\text { Consistency: Unknown } \\
\text { Directness: Direct } \\
\text { Imprecision: Imprecise }(p>0.05)\end{array}$ \\
\hline MNA-SF & $\begin{array}{l}\text { Discharge } \\
\text { Disposition }\end{array}$ & ICU & $\begin{array}{l}1 \text { SR, Lew } \\
\text { et al. } 2016^{31}\end{array}$ & $\begin{array}{l}1 \text { prospective } \\
\text { cohort }(260)\end{array}$ & $\begin{array}{l}\text { Inconclusive } \\
\text { Sheean et al. } 2013 \text {, adj } p=0.19 \\
\text { (no further details reported) }\end{array}$ & $\begin{array}{l}\text { Insufficient } \\
\text { Risk of bias: Moderate } \\
\text { Consistency: Unknown } \\
\text { Directness: Direct } \\
\text { Imprecision: Imprecise }(p>0.05)\end{array}$ \\
\hline
\end{tabular}




\begin{tabular}{|c|c|c|c|c|c|c|}
\hline Intervention & Outcome & Population & Reference & $\begin{array}{l}\text { Number and } \\
\text { Type of Study } \\
\text { (\# Patients) }\end{array}$ & Direction of Association/Findings & Overall SoE/Domains \\
\hline SNAQ & Mortality & ICU & $\begin{array}{l}1 \text { SR, Lew } \\
\text { et al. } 2016^{31}\end{array}$ & $\begin{array}{l}1 \text { prospective } \\
\text { cohort }(325)\end{array}$ & $\begin{array}{l}\text { Inconclusive } \\
\text { van Venrooji et al. 2011, p>0.05 } \\
\text { (no further details reported) }\end{array}$ & $\begin{array}{l}\text { Insufficient } \\
\text { Risk of bias: High } \\
\text { Consistency: Unknown } \\
\text { Directness: Direct } \\
\text { Imprecision: Imprecise }(\mathrm{p}>0.05)\end{array}$ \\
\hline SNAQ & Length of Stay & ICU & $\begin{array}{l}1 \text { SR, Lew } \\
\text { et al. } 2016^{31}\end{array}$ & $\begin{array}{l}1 \text { prospective } \\
\text { cohort }(325)\end{array}$ & $\begin{array}{l}\text { Inconclusive } \\
\text { van Venrooji et al. 2011, p>0.05 } \\
\text { (no further details reported) }\end{array}$ & $\begin{array}{l}\text { Insufficient } \\
\text { Risk of bias: High } \\
\text { Consistency: Unknown } \\
\text { Directness: Direct } \\
\text { Imprecision: Imprecise }(p>0.05)\end{array}$ \\
\hline SNAQ & HAC & ICU & $\begin{array}{l}1 \text { SR, Lew } \\
\text { et al. } 2016^{31}\end{array}$ & $\begin{array}{l}1 \text { prospective } \\
\text { cohort }(1,193)\end{array}$ & $\begin{array}{l}\text { Inconclusive } \\
\text { Lomivorotov et al. 2013, Post-operative } \\
\text { complications, AOR: } 1.30,95 \% \mathrm{Cl}: 0.90 \\
\text { to } 2.00, p=0.11\end{array}$ & $\begin{array}{l}\text { Insufficient } \\
\text { Risk of bias: Moderate } \\
\text { Consistency: Unknown } \\
\text { Directness: Direct } \\
\text { Imprecision: Imprecise }(p>0.05)\end{array}$ \\
\hline PINI & Mortality & ICU & $\begin{array}{l}1 \text { SR, Lew } \\
\text { et al. } 2016^{31}\end{array}$ & $\begin{array}{l}1 \text { prospective } \\
\text { cohort (83) }\end{array}$ & $\begin{array}{l}\text { Inconclusive In } \\
\text { Schlossmacher et al. 2002, p=0.49 } \\
\text { (no further details reported) }\end{array}$ & $\begin{array}{l}\text { Insufficient } \\
\text { Risk of bias: High } \\
\text { Consistency: Unknown } \\
\text { Directness: Direct } \\
\text { Imprecision: Imprecise }(p>0.05)\end{array}$ \\
\hline PINI & Length of Stay & ICU & $\begin{array}{l}1 \text { SR, Lew } \\
\text { et al. } 2016^{31}\end{array}$ & $\begin{array}{l}1 \text { prospective } \\
\text { cohort (83) }\end{array}$ & $\begin{array}{l}\text { Inconclusive } \\
\text { Schlossmacher et al. 2002, p>0.05 } \\
\text { (no further details reported) }\end{array}$ & $\begin{array}{l}\text { Insufficient } \\
\text { Risk of bias: High } \\
\text { Consistency: Unknown } \\
\text { Directness: Direct } \\
\text { Imprecision: Imprecise }(p>0.05)\end{array}$ \\
\hline
\end{tabular}

Note: Following AHRQ guidance, we rated the initial SOE as moderate if the authors of the reviews rated the ROB of included studies as low or moderate and indicated that studies conducted analyses to control for critical confounders, such as age and severity of illness.

$\mathrm{AHRQ}=$ Agency for Healthcare Research and Quality; ARM = at risk of malnutrition; AOR = adjusted odds ratio; $\mathrm{CI}=$ confidence intervals; HAC $=$ hospital acquired condition; $\mathrm{HR}=$ hazard ratio; $\mathrm{MN}=$ malnourished; ICU = intensive care unit; MNA = Mini Nutritional Assessment; MNA-SF = MNA-short form; MUST = Malnutrition Universal Screening Tool; NR = not reported; NRI = Nutritional Risk Index; NRS-2002 = Nutritional Risk Score, OR = odds ratio; PINI = Prognostic Inflammatory and Nutrition Index; $\mathrm{ROB}=$ risk of bias; $\mathrm{RR}=$ risk ratio; $\mathrm{SNAQ}=$ Short Nutritional Assessment Questionnaire; $\mathrm{SOE}=$ strength of evidence; $\mathrm{WN}=$ well-nourished 


\section{Malnutrition Among Patients Hospitalized for Decompensations of Chronic Disease}

The relationship between chronic disease and malnutrition is complex. Malnutrition is a consequence, complication, and cause of deterioration of many chronic illnesses. ${ }^{34}$ Chronic illnesses with an underlying condition of inflammation or oxidative stress have potential for increased risk of malnutrition because inflammation increases catabolism of amino acids in lean body mass and can ultimately reduce functionality. For instance, patients with heart failure (HF)related malnutrition often enter a vicious cycle of undernutrition, inflammation, and cachexia. ${ }^{34}$ Progression of this cycle begins with HF-related undernutrition, which leads to the exacerbation of fluid retention, inflammation, neurohormonal activation and, ultimately, further deterioration of nutritional status and poor prognosis.

We included 4 SRs (including 29 relevant unique studies) that focused on the association of malnutrition with clinical outcomes among patients hospitalized due to complications related to various chronic diseases, such as heart failure, ${ }^{32}$ cancer, ${ }^{33} \mathrm{COPD},{ }^{34}$ and liver transplant. ${ }^{35}$ Prevalence of malnutrition across studies included in these SRs ranged from 8 percnetto 90 percent, with prevalence varying depending on patient population and tool used to identify malnutrition (Table D-2, Appendix D). For instance, prevalence among patients with HF ranged from 16 percent to 90 percent in studies using the MNA and 22 percent to 48 percent among studies using the Geriatric Nutritional Risk Index (GNRI). Table 5 presents the SOE ratings for all assessed outcomes by measurement tool. The table is organized by measurement tool and outcome and includes columns indicating what study provided the data (reference), findings and direction of findings (e.g., no association, increased/decreased occurrence of outcome), and strength of evidence rating.

\section{Heart Failure}

One SR (Lin et al. 2016) included 11 studies assessing the association between malnutrition and hospital outcomes in patients with heart failure. ${ }^{32}$ Included studies measured malnutrition using MNA (5 studies), MNA-SF (2 studies), Nutritional Risk Index (NRI, 3 studies), and GNRI (4 studies). Four studies ( $n=472$; Bonilla et al. 2011; Sargento et al. 2013; Aggarwal et al. 2013; Suzuki et al. 2015) demonstrated that patients hospitalized with heart failure and diagnosed with malnutrition (using MNA) may be at increased risk of mortality compared to well-nourished patients with heart failure (pooled hazard ratio [HR]: 4.32, 95 percent CI: 2.30 to $8.11, \mathrm{I}^{2}=0.0$ percent, SOE: Low). Of note, 1 small study (Sargento et al. 2013) included non-hospitalized heart failure patients. Studies using NRI and GNRI to identify patients at risk of malnutrition also demonstrated that risk of malnutrition may be associated with increased mortality. For other outcomes measured in this review (e.g., length of stay [LOS] and 30-day readmission), evidence was either not reported or insufficient to draw any conclusions about the association between malnutrition and other clinical outcomes.

\section{Cancer}

One SR (Gupta et al. 2011) included 6 prospective cohort studies assessing whether malnutrition measured using SGA in cancer patients was associated with increased hospital length of stay. ${ }^{33}$ Five of these prospective cohort trials included a combined 1,930 patients of which a majority $(n=1,354)$ had gastrointestinal cancers (Wu et al. 2010; Wu et al. 2009; Wakahara et al. 2007; Shirodkar et al. 2005; Ulander et al. 1998). Pooled analysis showed that 
patients hospitalized with cancer and diagnosed with malnutrition (using SGA) may stay in the hospital on average up to 11 days longer than well-nourished patients (mean days: $\mathrm{WN}=13$;

$\mathrm{MN}=24, \mathrm{p}<0.05$ ). The remaining prospective cohort study (Laky et al. 2010, $\mathrm{n}=157$ ) of patients with presumed or proven gynecological cancer found a non-significant trend towards prolonged hospital length of stay in patients identified as malnourished versus well-nourished using patientgenerated SGA (PG-SGA).

\section{COPD}

One SR (Muscariotoli et al. 2018) included 2 studies that assessed the association between malnutrition and hospital readmission among patients hospitalized for complications related to COPD. ${ }^{34}$ One study used MNA to identify malnutrition (Benedik et al., 2005), while the other study used MUST (Steer et al., 2010). Evidence from both studies was rated insufficient due to high risk of bias and imprecision.

\section{Cirrhosis and Liver Transplant}

One SR (Ney et al. 2019 ${ }^{35}$ ) evaluated the association between malnutrition (diagnosed using the SGA) and clinical outcomes pre and post-liver transplant among patients hospitalized for cirrhosis. The SR included 3 studies which found that malnutrition may be associated with pretransplant mortality (RR: 2.40, 95percentCI: 1.16 to 4.96; Ciocirlan et al., 2017; Nunes et al., 2017; Alvaras et al., 2005). Another 3 studies found insufficient evidence to support an association between malnutrition (diagnosed using the SGA) and post-transplant mortality (Bakshi et al., 2016; Stephenson et al., 2001; and Pikul et al., 1994). Similarly, the evidence was insufficient to support an association between malnutrition and post-transplant length of stay (Stephenson et al., 2001; Pikul et al., 1994). 
Table 5. Strength of evidence ratings by measurement tool for patients with chronic diseases

\begin{tabular}{|c|c|c|c|c|c|c|}
\hline Intervention & Outcome & Population & Reference & $\begin{array}{l}\text { Number and } \\
\text { Type of Study } \\
\text { (Patients) }\end{array}$ & $\begin{array}{l}\text { Direction of } \\
\text { Association/Findings }\end{array}$ & Overall SoE/Domain \\
\hline MNA & Mortality & $\begin{array}{l}\text { Heart } \\
\text { Failure }\end{array}$ & $\begin{array}{l}1 \text { SR } \\
\text { Lin et al. } \\
2016^{32}\end{array}$ & $\begin{array}{l}3 \text { prospective, } \\
1 \text { retrospective } \\
\text { cohort trial }(472)\end{array}$ & $\begin{array}{l}\text { Increased } \\
\text { Pooled HR: } 4.32,95 \% \text { Cl: } 2.30 \text { to } \\
\text { 8.11, I²=0.0\%; Bonilla et al. } 2011 \text {; } \\
\text { Sargento et al. 2013; Aggarwal } \\
\text { et al. 2013; Suzuki et al. } 2015\end{array}$ & $\begin{array}{l}\text { Low } \\
\text { Risk of bias: Moderate } \\
\text { Consistency: Consistent } \\
\text { Directness: Direct } \\
\text { Precision: Imprecise (small sample size) }\end{array}$ \\
\hline MNA-SF & Mortality & $\begin{array}{l}\text { Heart } \\
\text { Failure }\end{array}$ & $\begin{array}{l}1 \text { SR } \\
\text { Lin et al. } \\
2016^{32}\end{array}$ & $\begin{array}{l}1 \text { prospective, } \\
1 \text { retrospective } \\
\text { cohort trial }(212)\end{array}$ & $\begin{array}{l}\text { Inconclusive } \\
\text { Pooled HR: } 3.56,95 \% \mathrm{Cl}: 1.41 \text { to } \\
\text { 9.00, I²=0.0\%; Yost et al. } 2014 ; \\
\text { Sargento et al. } 2013\end{array}$ & $\begin{array}{l}\text { Insufficient } \\
\text { Risk of bias: Moderate } \\
\text { Consistency: Consistent } \\
\text { Directness: Direct } \\
\text { Precision: Imprecise (small sample size) }\end{array}$ \\
\hline NRI & Mortality & $\begin{array}{l}\text { Heart } \\
\text { Failure }\end{array}$ & $\begin{array}{l}1 \text { SR } \\
\text { Lin et al. } \\
2016^{32}\end{array}$ & $\begin{array}{l}2 \text { prospective, } \\
1 \text { retrospective } \\
\text { cohort trial }(1,785)\end{array}$ & $\begin{array}{l}\text { Increased } \\
\text { Pooled HR: 2.08, 95\% Cl: } 1.60 \text { to } \\
\text { 2.71, I²=44\%; Aziz et al. } 2011 ; \\
\text { Al-Naijer et al. 2015; Gouya et al. } \\
2014\end{array}$ & $\begin{array}{l}\text { Low } \\
\text { Risk of bias: High (no report of confounder } \\
\text { analysis) } \\
\text { Consistency: Consistent } \\
\text { Directness: Direct } \\
\text { Precision: Precise }\end{array}$ \\
\hline GNRI & Mortality & $\begin{array}{l}\text { Heart } \\
\text { Failure }\end{array}$ & $\begin{array}{l}1 \text { SR } \\
\text { Lin et al. } \\
2016^{32}\end{array}$ & $\begin{array}{l}2 \text { prospective, } \\
1 \text { retrospective } \\
\text { cohort trial }(978)\end{array}$ & $\begin{array}{l}\text { Increased } \\
\text { Pooled HR: } 3.11,95 \% \text { Cl: } 1.69 \text { to } \\
\text { 5.74), I²=70\%; Narumi et al. } 2013 \text {; } \\
\text { Kinugasa et al. 2013; Kaneko et al. } \\
2015\end{array}$ & $\begin{array}{l}\text { Low } \\
\text { Risk of bias: High } \\
\text { Consistency: Consistent } \\
\text { Directness: Direct } \\
\text { Precision: Precise }\end{array}$ \\
\hline NRS-2002 & $\begin{array}{l}\text { Length of } \\
\text { Stay }\end{array}$ & $\begin{array}{l}\text { Heart } \\
\text { Failure }\end{array}$ & $\begin{array}{l}1 \mathrm{SR} \\
\text { Lin et al. } \\
2016^{32}\end{array}$ & $\begin{array}{l}1 \text { prospective } \\
\text { cohort trial (131) }\end{array}$ & $\begin{array}{l}\text { Inconclusive } \\
\text { OR: } 2.99,95 \% \mathrm{Cl}: 1.33 \text { to } 6.73 \text {; } \\
\text { Tevik et al. } 2014\end{array}$ & $\begin{array}{l}\text { Insufficient } \\
\text { Risk of bias: High } \\
\text { Consistency: Unknown } \\
\text { Directness: Direct } \\
\text { Precision: Imprecise (small sample size) }\end{array}$ \\
\hline
\end{tabular}




\begin{tabular}{|c|c|c|c|c|c|c|}
\hline Intervention & Outcome & Population & Reference & $\begin{array}{l}\text { Number and } \\
\text { Type of Study } \\
\text { (Patients) }\end{array}$ & $\begin{array}{l}\text { Direction of } \\
\text { Association/Findings }\end{array}$ & Overall SoE/Domain \\
\hline SGA & $\begin{array}{l}\text { Length of } \\
\text { Stay }\end{array}$ & Cancer & $\begin{array}{l}1 \text { SR; Gupta } \\
\text { et al. } 2011^{33}\end{array}$ & $\begin{array}{l}5 \text { prospective } \\
\text { cohort trials } \\
(1,930)\end{array}$ & $\begin{array}{l}\text { Increased } \\
\text { Mean days: WN=13; MN=24, } \\
\text { p<0.05; Wu et al. 2010; Wu et al. } \\
\text { 2009; Wakahara et al. 2007; } \\
\text { Shirodkar et al. 2005; Ulander } \\
\text { et al. } 1998 \\
\text { *Mean days calculated by ECRI }\end{array}$ & $\begin{array}{l}\text { Low } \\
\text { Risk of bias: High } \\
\text { Consistency: Consistent } \\
\text { Directness: Direct } \\
\text { Precision: Precise }\end{array}$ \\
\hline PG-SGA & $\begin{array}{l}\text { Length of } \\
\text { Stay }\end{array}$ & Cancer & $\begin{array}{l}1 \text { SR; Gupta } \\
\text { et al. } 2011^{33}\end{array}$ & $\begin{array}{l}1 \text { prospective } \\
\text { cohort trial (157) }\end{array}$ & $\begin{array}{l}\text { Inconclusive } \\
\text { AOR >5 days: } 5.28 \text { (0.98 to 28.5), } \\
p>0.05 \text {; Laky et al. } 2010\end{array}$ & $\begin{array}{l}\text { Insufficient } \\
\text { Risk of bias: Moderate } \\
\text { Consistency: Unknown } \\
\text { Directness: Direct } \\
\text { Precision: Imprecise }(p>0.05)\end{array}$ \\
\hline MNA & $\begin{array}{l}\text { Readmission } \\
\text { at } 6 \text { months }\end{array}$ & COPD & $\begin{array}{l}1 \mathrm{SR} ; \\
\text { Muscariotoli } \\
\text { et al. } 2018^{34}\end{array}$ & $\begin{array}{l}1 \text { prospective } \\
\text { cohort trial (108) }\end{array}$ & $\begin{array}{l}\text { Inconclusive } \\
\text { MN group } \\
\text { Readmitted within } 6 \text { months; } \\
\text { malnourished } 67 \% \text {; at-risk } 39 \% \text {; } \\
\text { well-nourished } 35 \% ; p=0.10 ; \\
\text { Benedik et al. } 2005\end{array}$ & $\begin{array}{l}\text { Insufficient } \\
\text { Risk of bias: High } \\
\text { Consistency: Unknown } \\
\text { Directness: Direct } \\
\text { Precision: Imprecise }(p>0.05)\end{array}$ \\
\hline MUST & $\begin{array}{l}\text { 30-day } \\
\text { Readmission }\end{array}$ & COPD & $\begin{array}{l}1 \mathrm{SR} ; \\
\text { Muscariotoli } \\
\text { et al. } 2018^{34}\end{array}$ & $\begin{array}{l}1 \text { prospective } \\
\text { cohort trial }(547)\end{array}$ & $\begin{array}{l}\text { Inconclusive } \\
\text { OR: } 1.71,95 \% \mathrm{Cl}: 1.04 \text { to } 2.83 \text {, } \\
p=0.034 ; \text { Steer et al. } 2010\end{array}$ & $\begin{array}{l}\text { Insufficient } \\
\text { Risk of bias: High } \\
\text { Consistency: Unknown } \\
\text { Directness: Direct } \\
\text { Precision: Imprecise (small sample size) }\end{array}$ \\
\hline SGA & $\begin{array}{l}\text { Mortality } \\
\text { (pre- } \\
\text { transplant) }\end{array}$ & Cirrhosis & $\begin{array}{l}1 \text { SR; Ney } \\
\text { et al. } 2019^{35}\end{array}$ & $\begin{array}{l}3 \text { prospective } \\
\text { cohort trials (277) }\end{array}$ & $\begin{array}{l}\text { Increased } \\
\text { Pooled RR: } 2.40,95 \% \text { Cl: } 1.16 \text { to } \\
\text { 4.96, I²=14\%; Ciocirlan et al. } 2017 \text {; } \\
\text { Nunes et al. } 2017 \text {; Alvaras et al. } \\
2005\end{array}$ & $\begin{array}{l}\text { Low } \\
\text { Risk of bias: Moderate } \\
\text { Consistency: Consistent } \\
\text { Directness: Direct } \\
\text { Precision: Imprecise (small sample size) }\end{array}$ \\
\hline
\end{tabular}




\begin{tabular}{|c|c|c|c|c|c|c|}
\hline Intervention & Outcome & Population & Reference & $\begin{array}{l}\text { Number and } \\
\text { Type of Study } \\
\text { (Patients) }\end{array}$ & $\begin{array}{l}\text { Direction of } \\
\text { Association/Findings }\end{array}$ & Overall SoE/Domain \\
\hline SGA & $\begin{array}{l}\text { Mortality } \\
\text { (post- } \\
\text { transplant) }\end{array}$ & Cirrhosis & $\begin{array}{l}1 \text { SR; Ney } \\
\text { et al. } 2019^{35}\end{array}$ & $\begin{array}{l}3 \text { prospective } \\
\text { cohort trials (153) }\end{array}$ & $\begin{array}{l}\text { Inconclusive } \\
\text { Bakshi et al. 2016: RR: } 0.66, \\
95 \% \text { Cl: } 0.10 \text { to } 4.55 ; \text { Stephenson } \\
\text { et al. 2001: RR: } 5.23,95 \% \text { Cl: } 1.07 \\
\text { to 25.54; Pikul et al. } 1994, \text { RR: } \\
\text { 5.73, 95\% Cl: } 0.36 \text { to } 97.2 \\
\end{array}$ & $\begin{array}{l}\text { Insufficient } \\
\text { Risk of bias: High } \\
\text { Consistency: Inconsistent } \\
\text { Directness: Direct } \\
\text { Imprecision: Imprecise (small sample size) }\end{array}$ \\
\hline SGA & $\begin{array}{l}\text { Length of } \\
\text { Stay }\end{array}$ & Cirrhosis & $\begin{array}{l}1 \text { SR; Ney } \\
\text { et al. } 2019^{35}\end{array}$ & $\begin{array}{l}2 \text { prospective } \\
\text { cohort trials (98) }\end{array}$ & $\begin{array}{l}\text { Inconclusive } \\
\text { Pooled RR: } 4.35,95 \% \text { Cl: } 5.42 \text { to } \\
\text { 22.69, I²=93\%; Pikul et al. 1994; } \\
\text { Stephenson et al. } 2001\end{array}$ & $\begin{array}{l}\text { Insufficient } \\
\text { Risk of bias: High } \\
\text { Consistency: Consistent } \\
\text { Directness: Direct } \\
\text { Precision: Imprecise (small sample size) }\end{array}$ \\
\hline
\end{tabular}

Note: Following AHRQ guidance, we rated the initial SOE as moderate if the authors of the reviews rated the ROB of included studies as low or moderate and indicated that studies conducted analyses to control for critical confounders, such as age and severity of illness.

$\mathrm{AOR}=$ adjusted odds ration; $\mathrm{CI}=$ confidence intervals; $\mathrm{HAC}=$ hospital acquired condition; $\mathrm{HR}=$ hazard ratio; $\mathrm{MNA}=$ Mini Nutritional Assessment;

MNA-SF = MNA-short form; MUST = Malnutrition Universal Screening Tool; NR = not reported; NRI = Nutritional Risk Index; NRS-2002 = Nutritional Risk Score,

$\mathrm{OR}=$ odds ratio; PINI = Prognostic Inflammatory and Nutrition Index; RR = risk ratio; SNAQ = Short Nutritional Assessment Questionnaire 
Key Question 2. What is the effectiveness of screening or diagnostic assessment for malnutrition among hospitalized adults?
a. In studies that report on clinical outcomes, what is the accuracy of screening or diagnostic tools for malnutrition?
b. In studies that report on clinical outcomes, what is the effectiveness of screening or diagnostic tools on measures of nutrition (nutritional stores)?
c. What is the impact of the use of screening or diagnostic tools on clinical outcomes?

\section{Description of Included Evidence}

Our literature searches found no studies that met inclusion criteria and addressed KQ 2 or its sub-questions.

We screened 38 abstracts of studies that potentially addressed KQ 2. Twenty-six were excluded for the following reasons: population not of interest, intervention not of interest, setting not of interest, addressed a different KQ, generally off-topic, and not in English. We reviewed 12 studies at the full text level and excluded 11 primarily for not having the appropriate study design to address the question. In most cases, studies were excluded at full-text level for not including an appropriate control. Appropriate controls for this KQ would include a non-exposed group of patients who did not receive screening or diagnostic assessment or a concurrent cohort of patients who received another screening or assessment tool (e.g., reference standard).

One prospective cohort study by Rypkema et al. $(2003)^{36}$ did compare diagnostic assessment of malnutrition to an appropriate control (no diagnostic assessment) with patients diagnosed with malnutrition subsequently receiving a nutritional intervention. However, in addition to including patients that screened positive for malnutrition, the study also included patients screening positive for additional diagnoses, such as dehydration or dysphagia, and failed to report outcomes for malnourished patients separately. We contacted the authors to request subgroup analysis results, but the data were no longer archived. Thus, this study was excluded.

\section{Key Points}

- We identified no studies that met inclusion criteria and addressed KQ 2 or its subquestions.

- The primary reason studies did not meet inclusion criteria for KQ 2 was failure to have an appropriate control group.

- Evidence gaps for KQ 2 underscore the need for future research that addresses the effectiveness of screening for malnutrition on clinical outcomes. Such research is vital to establish the role of inpatient screening and its downstream implications on patientrelevant outcomes.

\section{Summary of Findings}

The goal of KQ 2 was to assess the clinical utility of malnutrition measurement tools (i.e., screening or diagnostic assessments) for malnutrition. Specifically, this question was intended to 
determine whether performing malnutrition screening and/or diagnostic assessment prompts actions that change either nutritional stores or key clinical outcomes, such as mortality, length of stay, or hospital acquired complications.

Assessing the clinical utility of malnutrition measurement tools requires consideration of multiple study design factors, of which the most important are 1) inclusion of an appropriate control group and 2) reporting on changes in clinical outcomes. The most appropriate control for studies assessing the clinical utility of measurement tools would be patients who do not receive screening or diagnostic assessment. Without such a control group, it is not possible to determine if any observed study effects are attributable to the tool. In addition, to adequately assess clinical utility, studies would also need to report on changes in clinical outcomes. Thus, studies that are soley designed to measure diagnostic accuracy are not adequate for assessing clinical utility. Instead, diagnostic accuracy studies are focused on evaluating the sensitivity and specificity of a screening or diagnostic assessment tool compared with a reference standard, and typically do not include a non-exposed control group or report on associated clinical outcomes. Finally, other study designs, such as retrospective, before/after, and other non-prospective (or non-concurrent comparison) observational studies are problematic due to their potential for bias associated with confounder effects and patient selection.

Studies that would most directly inform KQ 2 would randomize hospitalized patients to a measurement tool vs. no measurement tool (See Figure 2 in the Methods section). However, in the US, hospital accreditation by The Joint Commission mandates screening. This existing mandate presents clear pragmatic challenges to randomizing U.S. patients to screening vs. no screening. Future trials could still randomize patients to different screening tools to assess the impact of various tools on clinical outcomes. Ideally, a study could screen all patients and randomize "at-risk" patients to SGA, no diagnostic assessment, or another tool (e.g., Global Leadership Initiative on Malnuttrition [GLIM]). Participants in both of these groups would then be identified as either malnourished, leading to an intervention, or not malnourished, resulting in no intervention or continued standard care. Another design could utilize all types of diagnostic assessments for "at-risk" patients, given that these assessments are non-invasive, and then randomize malnutrition interventions based on just one of these assessments. This would provide insight regarding the clinical course for patients who are false negatives and any potential harms of using specific tools.

Table 6 presents reasons studies were excluded at the full-text level for KQ 2. Several studies were screened that reported diagnostic accuracy and associated prognostic clinical outcomes. However, no studies were identified that allowed determination of the effectiveness of malnutrition screening or diagnostic assessment based on criteria presented in Figure 3 and described in our review protocol. While study design was the most common primary reason for exclusion, there were also important secondary reasons for exclusion. Aside from not having an appropriate control, eleven studies were also excluded because they did not include an appropriate reference standard comparator for diagnostic assessment. ${ }^{37}$ Of these, eight did not include any comparator, ${ }^{37}$ two used inappropriate reference standard comparators, ${ }^{38,39}$ and one utilized historic controls. ${ }^{40}$ No studies reviewed at the full-text level reported intermediate outcomes for direct measures of nutrition stores (KQ 2b).

Several randomized controlled trials (RCTs) that reached full-text review did not randomize patients to either a screening or diagnostic assessment tool, but instead randomized patients to an intervention for treatment after results of malnutrition testing in both arms were known, and were therefore included as part of KQ 3. ${ }^{41,42}$ Similarly, if studies at full-text level screening were 
nonrandomized, lacking a concurrent unexposed group, and reported only on association of malnutrition with outcomes, they were included in KQ 1 (if included within a systematic review). ${ }^{32,43,44}$ 
Table 6. Evaluation of PICOs of studies excluded at full-text level for Key Question 2

\begin{tabular}{|c|c|c|c|c|c|c|c|c|}
\hline Reference & $\begin{array}{l}\text { Tool Used } \\
\text { (Screening or } \\
\text { Diagnostic) }\end{array}$ & $\begin{array}{l}\text { Population: } \\
\text { Hospitalized } \\
\text { Patients } \\
\text { (Condition) }\end{array}$ & $\begin{array}{l}\text { Intervention: } \\
\text { Screened/Assessed } \\
\text { for MN (Tool Used) }\end{array}$ & $\begin{array}{l}\text { Control: } \\
\text { Unexposed } \\
\text { Arm }\end{array}$ & $\begin{array}{l}\text { Comparator: Used } \\
\text { Appropriate } \\
\text { Reference } \\
\text { Standard }\end{array}$ & $\begin{array}{l}\text { Intermediate } \\
\text { Outcome: } \\
\text { Diagnostic } \\
\text { Accuracy } \\
\text { Outcomes } \\
\text { (KQ 2a) }\end{array}$ & $\begin{array}{l}\text { Intermediate } \\
\text { Outcome: Direct } \\
\text { Measures of } \\
\text { Nutrition Stores } \\
\text { (KQ 2b) }\end{array}$ & $\begin{array}{l}\text { Outcomes: } \\
\text { Clinical } \\
\text { Outcomes }\end{array}$ \\
\hline $\begin{array}{l}\text { Zhang et al. } \\
2021^{45}\end{array}$ & $\begin{array}{l}\text { Screening and } \\
\text { diagnostic }\end{array}$ & $\begin{array}{l}\text { Yes (adult } \\
\text { patients with } \\
\text { cancer) }\end{array}$ & $\begin{array}{l}\text { Yes (NRS-2002, MUST, } \\
\text { PG-SGA, GLIM) }\end{array}$ & No* & No & Yes & No & No \\
\hline $\begin{array}{l}\text { Kroc et al. } \\
2021^{46}\end{array}$ & $\begin{array}{l}\text { Screening and } \\
\text { diagnostic }\end{array}$ & Yes & Yes (SGA, NRS-2002) & No* & No & Yes & No & Yes \\
\hline $\begin{array}{l}\text { Thelia et al. } \\
2020^{9}\end{array}$ & $\begin{array}{l}\text { Diagnostic } \\
\text { assessment }\end{array}$ & $\begin{array}{l}\text { Yes (critical } \\
\text { care patients) }\end{array}$ & Yes (GLIM) & No* & Yes (SGA) & Yes & No & No \\
\hline $\begin{array}{l}\text { Thomas } \\
\text { et al. } 2019^{38}\end{array}$ & $\begin{array}{l}\text { Screening and } \\
\text { diagnostic }\end{array}$ & $\begin{array}{l}\text { Yes (vascular } \\
\text { surgery } \\
\text { patients) }\end{array}$ & $\begin{array}{l}\text { Yes (MST, MUST, NRS- } \\
\text { 2002, MNA-SF) }\end{array}$ & No* & $\begin{array}{l}\text { No (Used PG-SGA } \\
\text { as reference } \\
\text { standard) }\end{array}$ & Yes & No & Yes \\
\hline $\begin{array}{l}\text { Becker et al. } \\
2019^{47}\end{array}$ & $\begin{array}{l}\text { Diagnostic } \\
\text { assessment }\end{array}$ & Yes & Yes (MNA) & No* & No & No & No & Yes \\
\hline $\begin{array}{l}\text { Omidvari } \\
\text { et al. } 2016^{48} \\
\text { Systematic } \\
\text { Review } \\
\end{array}$ & $\begin{array}{l}\text { Screening and } \\
\text { diagnostic }\end{array}$ & Yes & Yes (Multiple) & $\mathrm{NR}^{*}$ & NR & NR & NR & Yes \\
\hline $\begin{array}{l}\text { Lin et al. } \\
2016^{32} \\
\text { Systematic } \\
\text { Review }\end{array}$ & $\begin{array}{l}\text { Screening and } \\
\text { diagnostic }\end{array}$ & Yes & Yes (Multiple) & $\begin{array}{l}\mathrm{NR}^{*} \text { (included } \\
\text { in } \mathrm{KQ} \text { 1) }\end{array}$ & NR & NR & NR & Yes \\
\hline $\begin{array}{l}\text { Tapia et al. } \\
2015^{37}\end{array}$ & Screening only & Yes & Yes (NRI, GNRI) & No* & $\begin{array}{l}\text { Yes (SGA reported, } \\
\text { but study did not } \\
\text { use as reference } \\
\text { standard) }\end{array}$ & No & No & Yes \\
\hline $\begin{array}{l}\text { Olveira } \\
\text { et al. } 2013^{49}\end{array}$ & Screening only & Yes & Yes (NRI, GNRI) & $\mathrm{No}^{*}$ & $\begin{array}{l}\text { Yes (SGA reported } \\
\text { but study did not } \\
\text { use as reference } \\
\text { standard) }\end{array}$ & No & No & Yes \\
\hline
\end{tabular}




\begin{tabular}{|c|c|c|c|c|c|c|c|c|}
\hline Reference & $\begin{array}{l}\text { Tool Used } \\
\text { (Screening or } \\
\text { Diagnostic) }\end{array}$ & $\begin{array}{l}\text { Population: } \\
\text { Hospitalized } \\
\text { Patients } \\
\text { (Condition) }\end{array}$ & $\begin{array}{l}\text { Intervention: } \\
\text { Screened/Assessed } \\
\text { for MN (Tool Used) }\end{array}$ & $\begin{array}{l}\text { Control: } \\
\text { Unexposed } \\
\text { Arm }\end{array}$ & $\begin{array}{l}\text { Comparator: Used } \\
\text { Appropriate } \\
\text { Reference } \\
\text { Standard }\end{array}$ & $\begin{array}{l}\text { Intermediate } \\
\text { Outcome: } \\
\text { Diagnostic } \\
\text { Accuracy } \\
\text { Outcomes } \\
\text { (KQ 2a) }\end{array}$ & $\begin{array}{l}\text { Intermediate } \\
\text { Outcome: Direct } \\
\text { Measures of } \\
\text { Nutrition Stores } \\
\text { (KQ 2b) }\end{array}$ & $\begin{array}{l}\text { Outcomes: } \\
\text { Clinical } \\
\text { Outcomes }\end{array}$ \\
\hline $\begin{array}{l}\text { Lomivorotov } \\
\text { et al. } 2013^{43}\end{array}$ & $\begin{array}{l}\text { Screening and } \\
\text { diagnostic }\end{array}$ & $\begin{array}{l}\text { Yes } \\
\text { (scheduled } \\
\text { heart valve } \\
\text { surgery) }\end{array}$ & $\begin{array}{l}\text { Yes (SNAQ, MUST, } \\
\text { NRS-2002, MNA) }\end{array}$ & $\begin{array}{l}\text { No* (included } \\
\text { in } K Q \text { 1) }\end{array}$ & Yes (SGA) & Yes & No & Yes \\
\hline $\begin{array}{l}\text { Holyday } \\
\text { et al. } 2012^{41}\end{array}$ & $\begin{array}{l}\text { Screening and } \\
\text { diagnostic }\end{array}$ & Yes & Yes (MNA) & $\begin{array}{l}\text { No* (included } \\
\text { in } \mathrm{KQ} 3 \text { ) }\end{array}$ & No & No & No & Yes \\
\hline $\begin{array}{l}\text { Velasco } \\
\text { et al. } 2011^{44}\end{array}$ & $\begin{array}{l}\text { Screening and } \\
\text { diagnostic }\end{array}$ & Yes & $\begin{array}{l}\text { Yes (NRS-2002, MUST, } \\
\text { MNA) }\end{array}$ & No* & Yes (SGA) & Yes & No & Yes \\
\hline $\begin{array}{l}\text { Kruizenga } \\
\text { et al. } 2005^{40}\end{array}$ & Screening only & Yes & Yes (SNAQ) & $\begin{array}{l}\text { No* (Used } \\
\text { historical } \\
\text { controls) }\end{array}$ & $\begin{array}{l}\text { No (Used historical } \\
\text { controls) }\end{array}$ & No & No & Yes \\
\hline $\begin{array}{l}\text { Martins et al. } \\
2005^{39}\end{array}$ & $\begin{array}{l}\text { Screening and } \\
\text { diagnostic }\end{array}$ & Yes & $\begin{array}{l}\text { Yes (MNA, MUST, } \\
\text { SGA) }\end{array}$ & No* & $\begin{array}{l}\text { No (Used NRS as } \\
\text { reference standard) }\end{array}$ & No & No & Yes \\
\hline $\begin{array}{l}\text { Johansen } \\
\text { et al. } 2004^{42}\end{array}$ & Screening only & Yes & Yes (NRS-2002) & $\begin{array}{l}\text { No* (included } \\
\text { in } K Q \text { 3) }\end{array}$ & No & No & No & Yes \\
\hline $\begin{array}{l}\text { Rypkema } \\
\text { et al. } 2003^{36}\end{array}$ & $\begin{array}{l}\text { Diagnostic } \\
\text { assessment }\end{array}$ & Yes & $\begin{array}{l}\text { No* } \\
\text { Screened with MNA-SF, } \\
\text { but also included } \\
\text { screening for dysphagia } \\
\text { or dehydration }\end{array}$ & Yes & No* & No & No & Yes \\
\hline
\end{tabular}

* Primary reason for excluding study.

Legend: This table shows the key criteria for studies to meet inclusion for Key Question 2. The "control group" should be an unexposed cohort that did not receive the screening tool or diagnostic assessment. Appropriate reference standards include: For studies examining screening tools, the reference standard would be no screening or usual care, or a gold standard like imaging modalities to assess body composition or SGA. If a diagnostic tool was used, the reference standard should be radiographic imaging or SGA.

MNA = Mini Nutritional Assessment; MNA-SF = MNA-short form; MUST = Malnutrition Universal Screening Tool; NR = not reported; NRI = Nutritional Risk Index;

NRS-2002 = Nutritional Risk Score, PICO = Patient, Intervention, Comparator, Outcome; PINI = Prognostic Inflammatory and Nutrition Index; SNAQ = Short Nutritional Assessment Questionnaire 


\section{Key Question 3. Among patients diagnosed with malnutrition, what is the effectiveness of hospital-initiated interventions used to treat malnutrition on clinical outcomes?}

\section{Description of Included Evidence}

Our searches identified 11 RCTs $(n=3,748)$ that met inclusion criteria and evaluated hospitalinitiated interventions for patients hospitalized for various clinical conditions who were also identified as malnourished. For inclusion, all patients in these trials met criteria for malnutrition or at-risk for malnutrition as measured by one of the following tools: MNA, MUST, NRS-2002, PG-SGA, and SGA (see Table 7). One study assessed outcomes for well-nourished, at-risk, and malnourished patient subgroups separately. ${ }^{41}$ All other studies focused solely on either malnourished, at-risk, or combined at-risk and malnourished patients. Below, we briefly describe characteristics about the included studies. More detailed information is presented in Table D-3 in Appendix D.

\section{Study Characteristics}

Patients enrolled in the included trials were hospitalized for a variety of conditions including cancer, cardiovascular disease, gastrointestinal disorder, heart failure, infectious disease, neurological disorder, pulmonary disease, renal disease, or stroke. Although most studies did not specify an admitting diagnosis for inclusion, two studies only included patients hospitalized for heart failure ${ }^{50}$ or stroke. $^{51}$ Three studies had minimum age requirements for inclusion of $60,{ }^{52}$ $65,{ }^{53}$ and $75 .{ }^{54}$ Four studies were large multicenter clinical trials. ${ }^{42,50,53,55}$ Only one study (Deutz et al.) was performed in the U.S. ${ }^{53}$ Of the remaining 10 studies, 2 were performed in Australia and the remainder in Europe. Study participants were generally older (mean age of patients ranged from 62 to 83.2). Mean BMI at admission ranged from 19.0 to 26.9, and mean weight ranged from $52.6 \mathrm{~kg}$ to $70.9 \mathrm{~kg}$. Only one study reported on race and socioeconomic status. ${ }^{53}$

\section{Interventions}

Included RCTs assessed two types of interventions: specialized nutrition care and increased protein/calorie provision (see Table 8). Eight trials $(n=2,965)$ assessed specialized nutrition care where patients received individualized consultation from nutrition specialists for goal setting and increasing patients' protein and calorie consumption during hospitalization. ${ }^{41,42,50-52,55-57}$ The other 3 trials assessed increasing protein/calorie provision either through a high protein/high calorie oral supplement (containing beta-hydroxybeta-methylbutyrate) ${ }^{53}$ a high protein/high calorie liquid supplement (with $500 \mathrm{kcal}$ and 21 grams of protein), ${ }^{54}$ or through protein and calorie fortified meal choices. ${ }^{58}$ The control group in 9 of 11 studies was usual care without any modifiers to routine clinical practice. ${ }^{41,42,50-52,54-56,58}$ Usual care in four studies could include a malnutrition care plan, ${ }^{41}$ dietary consultation, ${ }^{52}$ an oral nutrition supplement prescription, ${ }^{56}$ or tube feeding ${ }^{51}$ at the discretion of the provider. The remaining five studies provided no details describing usual care. ${ }^{42,50,54,55,58}$ In one study of high protein/calorie nutritional supplementation, the control group received a placebo without other nutritional interventions. ${ }^{53}$ In another study of specialized nutrition care, ${ }^{57}$ patients were provided usual care along with an energy-dense supplement but not given any further nutritional instructions. 
Of note, no studies assessing parenteral or enteral support interventions met criteria for inclusion, primarily because studies assessing these interventions did not restrict enrollment to patients who were diagnosed as at-risk of malnutrition or malnourished based on commonly available measurement tools. Instead, these studies relied on ICU admission, surgical status, or biometrics (e.g., BMI, serum albumin levels) as a proxy for malnutrition status.

Table 7. Characteristics of studies assessing the effect of hospital-initiated intervention to treat malnutrition

\begin{tabular}{|c|c|c|c|}
\hline Study & $\begin{array}{l}\text { Enrollment Requirement } \\
\text { Malnutrition Status Based on } \\
\text { Score }\end{array}$ & Intervention & Risk of Bias \\
\hline $\begin{array}{l}\text { Bonilla-Palomas } \\
2016^{50}\end{array}$ & $\begin{array}{l}\text { Malnourished } \\
\text { MNA score }<17\end{array}$ & Specialized Nutrition Care & Some Concerns \\
\hline Deutz $2016^{53}$ & $\begin{array}{l}\text { At-Risk \& Malnourished } \\
\text { SGA score B or C }\end{array}$ & $\begin{array}{l}\text { Protein/Calorie } \\
\text { Supplementation }\end{array}$ & Some Concerns \\
\hline Gazzotti $2003^{54}$ & $\begin{array}{l}\text { At-Risk } \\
\text { MNA score between } 17 \text { and } 23.5\end{array}$ & $\begin{array}{l}\text { Protein/Calorie } \\
\text { Supplementation }\end{array}$ & Some Concerns \\
\hline Ha $2010^{51}$ & $\begin{array}{l}\text { At-Risk \& Malnourished } \\
\text { MUST score }>0\end{array}$ & Specialized Nutrition Care & High \\
\hline $\begin{array}{l}\text { Holyday } 2011^{41} \\
\text { At Risk }\end{array}$ & $\begin{array}{l}\text { At-Risk } \\
\text { MNA score between } 17 \text { and } 23.5\end{array}$ & Specialized Nutrition Care & High \\
\hline $\begin{array}{l}\text { Holyday } 2011^{41} \\
\text { Malnourished }\end{array}$ & $\begin{array}{l}\text { Malnourished } \\
\text { MNA score }<17\end{array}$ & Specialized Nutrition Care & High \\
\hline Johansen $2004^{42}$ & $\begin{array}{l}\text { At-Risk \& Malnourished } \\
\text { NRS } 2002 \text { score } \geq 3\end{array}$ & Specialized Nutrition Care & High \\
\hline Munk $2014^{57}$ & $\begin{array}{l}\text { At-Risk \& Malnourished } \\
\text { NRS } 2002 \text { score } \geq 3\end{array}$ & $\begin{array}{l}\text { Protein/Calorie } \\
\text { Supplementation }\end{array}$ & Some Concerns \\
\hline Rufenacht $2011^{58}$ & $\begin{array}{l}\text { At-Risk \& Malnourished } \\
\text { NRS } 2002 \text { score } \geq 3\end{array}$ & Specialized Nutrition Care & $\begin{array}{l}\text { Some Concerns (mortality) } \\
\text { High (other relevant outcomes) }\end{array}$ \\
\hline Schuetz $2019^{55}$ & $\begin{array}{l}\text { At-Risk \& Malnourished } \\
\text { NRS } 2002 \text { score } \geq 3\end{array}$ & Specialized Nutrition Care & $\begin{array}{l}\text { Low (objective outcomes) } \\
\text { High (quality of life) }\end{array}$ \\
\hline Sharma $2017^{52}$ & $\begin{array}{l}\text { At-Risk \& Malnourished } \\
\text { PG SGA score B or C }\end{array}$ & Specialized Nutrition Care & High \\
\hline Starke $2011^{56}$ & $\begin{array}{l}\text { At-Risk \& Malnourished } \\
\text { NRS } 2002 \text { score } \geq 3\end{array}$ & Specialized Nutrition Care & $\begin{array}{l}\text { Some Concerns (objective } \\
\text { outcomes) } \\
\text { High (quality of life) }\end{array}$ \\
\hline
\end{tabular}

Note: Due to the paucity of literature identified for Key Question 3 in which patients were diagnosed with malnutrition using diagnostic assessment tools (such as Subjective Global Assessment [SGA] or Mini Nutritional Assessemnt [MNA]), we accepted studies in which patients were identified as at-risk of malnutrition using cutoff scores on screening tools (such as NRS-2002 and MUST). 


\section{Risk of Bias Ratings}

We assessed ROB separately for objective (e.g., mortality, readmissions, and length of stay) and subjective (e.g., quality of life) outcomes given differences in how these outcomes are measured. Domain level ROB, such as adequate randomization and allocation concealment, missing outcome data, and appropriate outcome measurement, informed overall ROB rating for each outcome. An overall ROB rating of low meant all domain level assessments were low, whereas a rating of high indicated that at least one domain level assessment was high. If a study contained a combination of low and some concerns for domain level assessments, the rater decided between an overall ROB of some concerns or high based on how the domain level assessment impacts confidence in the result. For one study, ${ }^{52}$ we assessed risk of bias for readmissions separately from other objective outcomes because, in addition to the dietary intervention, the intervention group also received monthly telehealth calls. These telehealth visits after initial dietary consultation reinforced the intervention and potentially impacted outcomes. Table 7 summarizes the ROB across studies, and Table E-2 in Appendix E provides detailed ROB ratings at the study and outcome level.

Overall, a large proportion of studies ( 7 of 11 studies) were rated high risk of bias for at least one outcome. ${ }^{41,42,51,52,55-57}$ The remaining studies (4 of 11) contained at least one outcome with some concerns. ${ }^{50,53,54,58}$ Studies with domains rated as "some concerns" had problems such as failing to report adequate randomization, unknown allocation concealment techniques, or lack of clarity regarding whether all pre-specified outcomes were reported. In addition to these problems, studies with outcomes rated as "high" risk of bias had problems such as high dropout (over 20 percent), inadequate blinding of outcome assessors, or use of subgroup analyses to highlight statistically significant results.

\section{Key Points}

- Hospital-initiated malnutrition interventions (specialized nutrition care, protein/calorie supplementation) likely decrease mortality compared to usual care. (SOE: Moderate)

- Hospital-initiated malnutrition interventions may improve quality of life compared to usual care. (SOE: Low)

- No difference was observed between hospital-initiated malnutrition interventions and usual care for length of stay, readmission rates, and hospital acquired conditions compared to usual care. (SOE: Low)

- Evidence was insufficient to address the effect of hospital-initiated malnutrition interventions on activities of daily living and discharge disposition compared to usual care. (SOE: Insufficient)

\section{Summary of Findings}

Compared to usual care, we found moderate quality evidence that hospital-initiated malnutrition interventions improved hospital-based mortality and low-quality evidence that it improves quality of life. For all other outcomes of interest, the evidence showed either no difference between groups or was considered insufficient due to study limitations and inconsistencies in the reported findings. Strength of evidence ratings for all outcomes considered are presented in Table 8 . One study ${ }^{50}$ was discontinued early because interim analysis determined a large beneficial effect. All other studies had no detected risk of reporting bias. We 
describe findings by specific outcomes below. All study level findings are presented in Table F-2 in Appendix F, and all meta-analytic results are presented in Figures 4 and 5, as well as Figures G-1 to G-13 in Appendix G.

\section{Mortality}

All 11 studies reported on mortality with follow-up ranging from 7 days to 6 months. Pooled analysis of these studies found malnutrition-focused interventions reduced mortality by 21 percent when compared to usual care (RR: $0.79,95$ percent $\mathrm{CI}$ : 0.63 to $0.98, \mathrm{I}^{2}=0.0$ percent). ${ }^{41,42,50-58}$ The pooled estimate was driven largely by one study, ${ }^{55}$ which contributed nearly two-thirds of the weight (See Figure 4). This multicenter Swiss study (Schuetz et al. 2019) enrolled over 1,000 hospitalized patients per arm with a range of primary diagnoses including cancer, cardiovascular disease, and infection and meeting criteria for at-risk for malnutrition or malnourished. Patients were followed for one month, and the study was rated low risk of bias for objective outcomes. Compared to patients randomized to usual care (standard hospital food), the study found that nutritional support (creating individualized targets and strategies to achieve targets within 48 hours of admission) was associated with a 27 percent lower mortality risk (RR: $0.73,95$ percent CI: 0.55 to 0.97 ), similar to the pooled estimate.

Figure 4. Effect of hospital-initiated interventions on mortality, all studies

\begin{tabular}{|c|c|c|c|c|c|c|c|}
\hline \multirow[b]{2}{*}{ Study } & \multirow[b]{2}{*}{ Events } & \multicolumn{2}{|l|}{$\mathrm{n}$} & \multirow{2}{*}{ Comparator } & & \multirow{2}{*}{$\begin{array}{l}\text { Risk Ratio } \\
(95 \% \mathrm{Cl})\end{array}$} & \multirow{2}{*}{$\begin{array}{r}\% \\
\text { Weight }\end{array}$} \\
\hline & & Intervention & Events & & & & \\
\hline Bonilla Palomas 2016 & 4 & 61 & 6 & $\rightarrow$ & 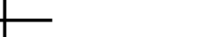 & $0.64(0.19,2.17)$ & 3.34 \\
\hline Deutz 2016 & 9 & 309 & 19 & 313 & & $0.48(0.22,1.04)$ & 8.15 \\
\hline Gazzotti 2003 & 2 & 39 & 2 & 41 & - & $1.05(0.16,7.10)$ & 1.35 \\
\hline Ha 2010 & 12 & 84 & 10 & 86 & + & $1.23(0.56,2.69)$ & 8.02 \\
\hline Holyday 2011 At Risk & 1 & 47 & 0 & 40 & & $2.56(0.11,61.21)$ & 0.49 \\
\hline Holyday 2011 Malnourished & 2 & 12 & 1 & 20 & & $3.33(0.34,32.96)$ & 0.94 \\
\hline Johansen 2004 & 9 & 108 & 6 & 104 & - & $1.44(0.53,3.92)$ & 4.95 \\
\hline Munk 2014 & 1 & 41 & 1 & 40 & & $0.98(0.06,15.07)$ & 0.66 \\
\hline Rufenacht 2011 & 4 & 18 & 1 & 18 & & $4.00(0.49,32.39)$ & 1.13 \\
\hline Schuetz 2019 & 73 & 1015 & 100 & 1013 & & $0.73(0.55,0.97)$ & 59.01 \\
\hline Sharma 2017 & 12 & 78 & 14 & 70 & & $0.77(0.38,1.55)$ & 10.04 \\
\hline Starke 2011 & 2 & 66 & 5 & 66 & & $0.40(0.08,1.99)$ & 1.91 \\
\hline \multirow[t]{3}{*}{ Overall $(1-$ squared $=0.0 \%)$} & & & & 1 & & $0.79(0.63,0.98)$ & 100.00 \\
\hline & & & & $\underbrace{}_{.015625}$ & 1 & & \\
\hline & & & & Favors Intervention & Favors Control & & \\
\hline
\end{tabular}

Subgroup analyses were performed on malnutrition status, follow-up time, and type of treatment (See Appendix G, Figures G2-G4). When examining each type of intervention, there were reductions of 18 percent and 44 percent in mortality risk for specialized nutrition care and protein/calorie supplements, respectively, but the findings were no longer statistically significant. When examining different follow-up times, there were significant decreases in one-month (postdischarge) mortality risk. Both inpatient mortality and greater than one-month (post-discharge) mortality risk were not different between treatments. However, findings for these subgroup 
analyses are impacted by low statistical power and small sample sizes. Therefore, these results should be interpreted with these limitations in mind.

\section{Length of Stay}

Nine out of 11 studies reported on length of stay. Pooled analysis of 9 studies showed a nonsignificant decrease of 0.18 days (weighted mean difference [WMD]: $-0.18,95$ percent CI:

-0.55 to 0.19 , SOE: Low $)^{41,42,51-56,58}$ This pooled estimate was driven largely by two studies. ${ }^{53,55}$

One study (Schuetz et al.) compared individualized consultation with a dietitian against usual care and found a non-significant 0.10 day decrease in length of stay (WMD: $-0.10,95$ percent CI: -0.67 to 0.47 ). The other study (Deutz et al.) randomized patients to receive an oral high protein/high calorie supplement or placebo and also found a non-significant 0.10 day decrease in length of stay (WMD: $-0.10,95$ percent CI: -0.64 to 0.44 ).

\section{Readmissions}

Seven out of 11 studies reported on readmissions with follow-up times ranging from 1 month to 6 months. The pooled estimate for readmission rates showed a non-significant decrease of 10 percent (RR: 0.90 , 95 percent CI: 0.74 to 1.09 ) among patients who received a malnutritionfocused intervention. ${ }^{41,50,52-56}$ The majority of the meta-analytic weight (around 85 percent) was based on 3 studies. Two of these studies specifically examined 30-day readmission rates, ${ }^{53,55}$ whereas the other study ${ }^{56}$ examined 6-month readmission. Since this was the follow-up time point with available data, we used this datapoint for our meta-analysis examining readmission. All individual studies contributing to the pooled estimate showed no significant difference.

\section{Quality of Life}

Six studies assessed the impact of specialized nutrition care on quality of life in at-risk and malnourished hospitalized patients. Four studies captured quality of life using the EuroQol five-dimension scale (EQ-5D), ${ }^{51,52,55,57}$ and 2 studies used the 36-Item Short Form Health Survey (SF-36). ${ }^{42,56}$ Study follow-up ranged from 1 to 3 months. Also, three studies reported change in quality of life from baseline, ${ }^{42,53,57}$ whereas the other three reported quality of life scores at discharge. ${ }^{52,55,56}$ Meta-analysis of data from 5 studies grouped by EQ-5D visual analogue scale (VAS) score or SF-36 physical component score (PCS) are shown in Figure 5. One study was not included due to inadequately reported data. ${ }^{51}$ Pooled analysis found that compared to usual care, patients receiving specialized nutrition care had improved quality of life (standardized mean difference $0.14,95$ percent CI: 0.06 to 0.22 , SOE: Low). 
Figure 5 . Effect of hospital-initiated interventions on quality of life, subgroup quality of life
measurement tool

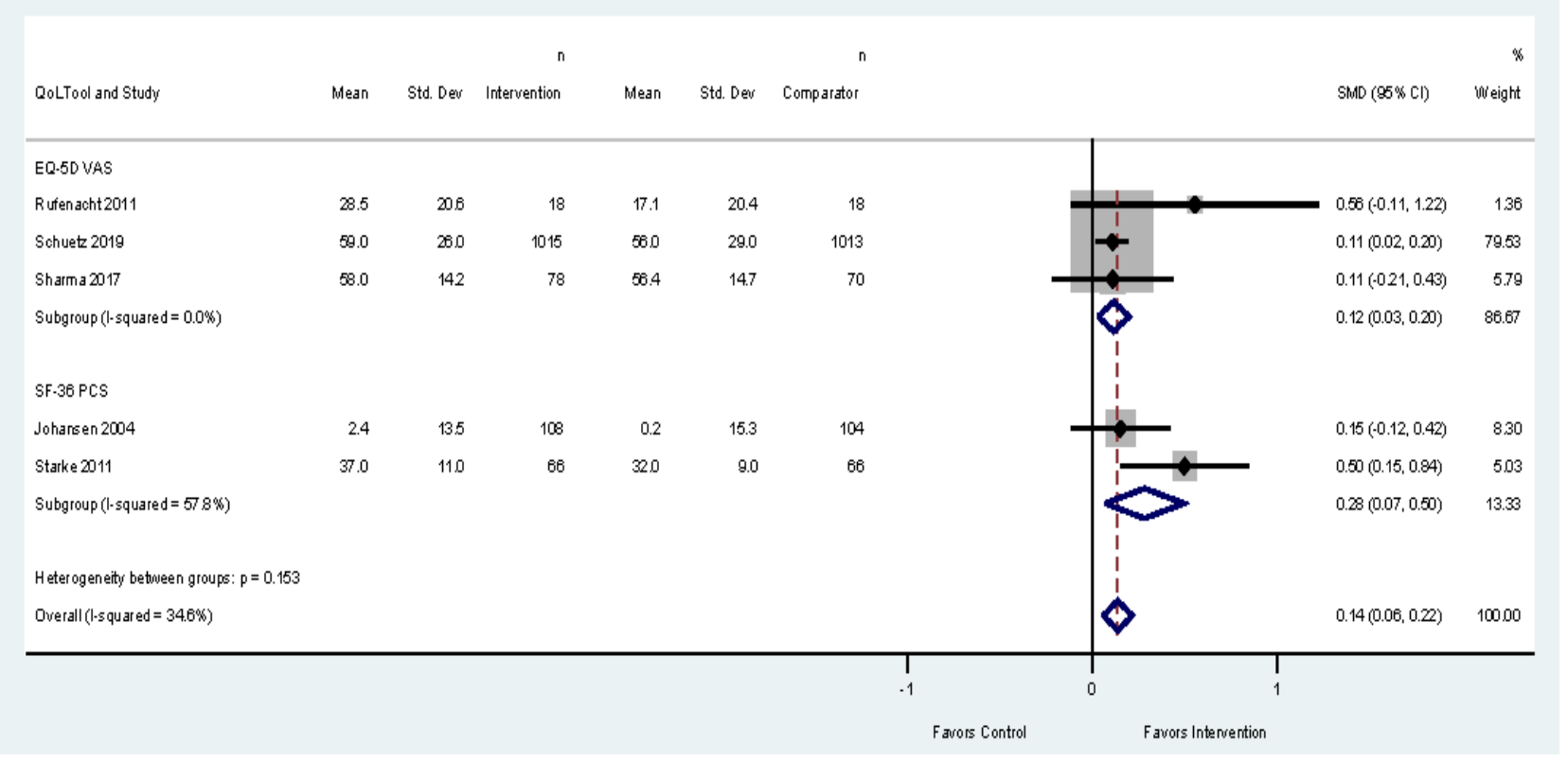

$\mathrm{CI}=$ confidence interval; EQ-5D; VAS = EuroQol 5 dimensional scale visual analogue scale; $\mathrm{n}=$ number of patients; SF-36 PCS $=36$ item short form health survey physical component score; SMD = standardized mean difference

\section{Activities of Daily Living}

Two studies (Schuetz et al. 2019, Deutz et al. 2016) reported impact of hospital-initiated malnutrition interventions on activities of daily living (ADLs) ${ }^{53,55}$ Both studies included at-risk and malnourished patients. Schuetz et al. found statistically significant improvement in functional status (measured by the Barthel Index) for patients randomized to specialized nutrition care at 1 month (compared to patients receiving standard hospital food) ${ }^{55}$ However, a second, smaller study (Deutz et al) of 622 patients older than age 65 found that, compared to placebo, patients receiving an oral protein/calorie supplement had similar changes in ADLs (measured by Katz scores, $p$ value not reported) at 1 month. ${ }^{53}$ Given these mixed results and lack of any additional data to support pooled analysis, we judged the evidence insufficient to permit a conclusion.

\section{Other Outcomes}

Included studies also described the effect of hospital-initiated malnutrition interventions on hospital acquired conditions (HACs), adverse events, and discharge disposition. Designation of HACs or adverse events were based on author descriptions. Four studies reported the impact of hospital-initiated malnutrition interventions on HACs (secondary medical events or illnesses occurring during a hospital stay [e.g., acute kidney failure, infection, and stroke]). Three studies slightly favored the intervention on incidence of HACs. ${ }^{52,55,56}$ The other study (Johansen et al. 2004) had no HACs in its control group, producing a very high relative risk score in favor of the control. ${ }^{42}$ A pooled estimate of 4 studies with low strength of evidence found interventions were not associated with a statistically significant difference in HACs compared with usual care (RR: $0.89,95$ percent CI: 0.69 to 1.14$){ }^{42,52,55,56}$

Three studies described the impact of hospital-initiated malnutrition interventions on occurrence of potentially treatment related adverse events (e.g. constipation, diarrhea, 
hyperglycemia, and refeeding syndrome); these studies reported no difference in the number of patients experiencing adverse events across patients receiving intervention compared to the control arm (usual care). ${ }^{53-55}$ Finally, only 2 studies reported the impact of hospital-initiated malnutrition interventions on discharge disposition. One study found no difference in patients discharged home (66.7 percent vs. 65 percent $),{ }^{54}$ while the other study found no difference in patients discharged to a residential facility $(7.7$ percent vs 8.5 percent, $p=0.09) .{ }^{52}$ Evidence was insufficient to draw a conclusion regarding the effect of hospital-initiated malnutrition interventions on discharge disposition.

\section{Comparison With Prior SRs}

Two previous systematic reviews examined the impact of nutrition-focused interventions on hospitalized patients. ${ }^{59,60}$ The most comprehensive review, Feinberg et al. (2017) included 244 RCTs. ${ }^{59}$ Most studies included in this review did not meet our inclusion criteria due to publication date before 2000 (130 RCTs), at risk or malnourished status not confirmed by a commonly available measurement tool (105 RCTs), measurement tools not utilized in the United States, Australia, New Zealand, Canada, or Europe (2 RCTs), or not a comparison group of interest (1 RCT). Instead of using a commonly available measurement tool, many studies included in this review used ICU admission, surgical status, or biometrics (e.g., BMI, serum albumin, or weight loss) to identify patients as malnourished.

Only 6 studies included in Feinberg et al. 2017 met our criteria and were included. These studies examined mortality, quality of life, serious adverse events, BMI change, and weight change. Like our review, Feinberg and colleagues found that nutrition-focused interventions reduced mortality (RR: $0.94,95$ percent CI: 0.86 to 1.03 ). However, our review found a larger magnitude of effect which was also statistically significant. This difference may be in part because of a large multicenter study (Schuetz et al. 2019, 2,028 patients) published subsequent to the Feinberg review and included in ours. ${ }^{55}$

Another systematic review by Gomes et al. (2019) included 27 randomized and nonrandomized controlled trials (published 1982 to 2019) evaluating the effectiveness of various nutritional support interventions (including dietary advice, changes in the organization of nutritional care, food fortification, extra snacks, oral nutrition supplements, and enteral tube feeding) used to treat non-critically ill hospitalized patients who were malnourished or at nutritional risk. ${ }^{60}$ Patients were determined to be malnourished or at nutritional risk based on body mass index, the presence of a disease associated with malnutrition, or the use of a nutritional measurement tool. The review's primary outcome was mortality, but the review also considered secondary outcomes such as length of stay, readmission, functional outcomes, infection, protein/calorie intake, and weight change. A total of 8 studies overlapped between our review and Gomes's review. Generally, findings for mortality, length of stay, activities of daily living, and rates of infection were similar to our results. However, our review found that hospital-initiated malnutrition interventions were not associated with a statistically significant difference in readmission rates, while Gomes found a reduction. 
Table 8. Strength of evidence ratings for interventions for malnutrition in patients diagnosed with malnutrition

\begin{tabular}{|c|c|c|c|c|c|c|c|c|}
\hline $\begin{array}{l}\text { Intervention/ } \\
\text { Comparator }\end{array}$ & $\begin{array}{l}\text { Outcome/ } \\
\text { Followup }\end{array}$ & $\begin{array}{l}\text { No Studies/No } \\
\text { Patients }\end{array}$ & Direction/Findings & $\begin{array}{l}\text { Risk of } \\
\text { Bias }\end{array}$ & Consistency & Precision & Directness & Overall SoE \\
\hline $\begin{array}{l}\text { Any nutrition } \\
\text { intervention } \\
\text { versus usual } \\
\text { care or placebo }\end{array}$ & $\begin{array}{l}\text { Mortality } \\
7 \text { days to } \\
6 \text { months }\end{array}$ & $\begin{array}{l}11 \mathrm{RCTs}^{41,42,50-58} \\
\mathrm{n}=3,748\end{array}$ & $\begin{array}{l}\text { Decreased } \\
\text { Pooled Estimate (11 studies): } \\
\text { RR: } 0.79(95 \% \mathrm{Cl}: 0.63 \text { to } 0.98) \\
\text { Overall } \mathrm{I}^{2}=0.0 \%\end{array}$ & Moderate & Consistent & Precise & Direct & Moderate \\
\hline $\begin{array}{l}\text { Any nutrition } \\
\text { intervention } \\
\text { versus usual } \\
\text { care or placebo }\end{array}$ & Length of Stay & $\begin{array}{l}9 \mathrm{RCTS}^{41,42,51-56,58} \\
\mathrm{n}=3,592\end{array}$ & $\begin{array}{l}\text { No difference } \\
\text { Pooled Estimate (9 studies): } \\
\text { WMD: }-0.18 \text { ( } 95 \% \mathrm{Cl}:-0.55 \text { to } \\
\text { 0.19), Overall } \mathrm{I}^{2}=35.5 \%\end{array}$ & Moderate & Consistent & Imprecise & Direct & Low \\
\hline $\begin{array}{l}\text { Any nutrition } \\
\text { intervention } \\
\text { versus usual } \\
\text { care or placebo }\end{array}$ & $\begin{array}{l}\text { Readmissions } \\
1 \text { month to } \\
6 \text { months }\end{array}$ & $\begin{array}{l}7 \mathrm{RCTs}^{41,50,52-56 *} \\
n=3,249\end{array}$ & $\begin{array}{l}\text { No Difference } \\
\text { Pooled Estimate (7 studies): } \\
\text { RR: } 0.90 \text { ( } 95 \% \mathrm{Cl}: 0.74 \text { to } 1.09), \\
\text { Overall } \mathrm{I}^{2}=8.1 \%\end{array}$ & Moderate & Consistent & Imprecise & Direct & Low \\
\hline $\begin{array}{l}\text { Any nutrition } \\
\text { intervention } \\
\text { versus usual } \\
\text { care }\end{array}$ & $\begin{array}{l}\text { Quality of Life } \\
1 \text { month to } \\
3 \text { months }\end{array}$ & $\begin{array}{l}6 \mathrm{RCTs}^{42,51,52,55-57} \\
\mathrm{n}=2,726\end{array}$ & $\begin{array}{l}\text { Increased } \\
\text { Pooled Estimate (5 studies): } \\
\text { SMD: } 0.14 \text { ( } 95 \% \mathrm{Cl}: 0.06 \text { to } 0.22) \text {, } \\
\text { Overall I }{ }^{2}=34.6 \%\end{array}$ & High & Consistent & Precise & Direct & Low \\
\hline $\begin{array}{l}\text { Any nutrition } \\
\text { intervention } \\
\text { versus usual } \\
\text { care or placebo }\end{array}$ & $\begin{array}{l}\text { Activities of } \\
\text { Daily Living } \\
3 \text { months }\end{array}$ & $\begin{array}{l}2 \operatorname{RCTs}^{53,55} \\
n=2,650\end{array}$ & $\begin{array}{l}\text { Inconclusive } \\
\text { Barthel index favors intervention; } \\
\text { Decline in functional status of } \\
\geq 10 \% \text { : favors intervention (1 RCT; } \\
n=2,028) \\
\text { Katz Score: Tx: } 6(\mathrm{Q} 16, \mathrm{Q} 36) \text {; } \\
\text { CG: } 6(6,6)(\mathrm{n}=622 ; 1 \mathrm{RCT}) \\
\mathrm{p} \text { value NR }\end{array}$ & Low & Inconsistent & $\begin{array}{l}\text { Imprecise (no } \\
\text { information on } \\
95 \% \text { Cls of } \\
\text { one of two } \\
\text { estimates) }\end{array}$ & Direct & Insufficient \\
\hline $\begin{array}{l}\text { Any nutrition } \\
\text { intervention } \\
\text { versus usual } \\
\text { care }\end{array}$ & $\begin{array}{l}\text { Hospital } \\
\text { Acquired } \\
\text { Conditions } \\
1 \text { month to } \\
3 \text { months }\end{array}$ & $\begin{array}{l}4 \operatorname{RCTs}^{42,52,55,56} \\
n=2,520\end{array}$ & $\begin{array}{l}\text { No Difference } \\
\text { Pooled Estimate (4 studies): } \\
\text { RR: } 0.89 \text { (95\% Cl: } 0.69 \text { to } 1.14) \text {, } \\
\text { Overall } I^{2}=57.5 \%\end{array}$ & Moderate & Consistent & Imprecise & Direct & Low \\
\hline
\end{tabular}




\begin{tabular}{|c|c|c|c|c|c|c|c|c|}
\hline $\begin{array}{l}\text { Intervention/ } \\
\text { Comparator }\end{array}$ & $\begin{array}{l}\text { Outcome/ } \\
\text { Followup }\end{array}$ & $\begin{array}{l}\text { No Studies/No } \\
\text { Patients }\end{array}$ & Direction/Findings & $\begin{array}{l}\text { Risk of } \\
\text { Bias }\end{array}$ & Consistency & Precision & Directness & Overall SoE \\
\hline $\begin{array}{l}\text { Any nutrition } \\
\text { intervention } \\
\text { versus usual } \\
\text { care }\end{array}$ & $\begin{array}{l}\text { Discharge } \\
\text { Disposition } \\
2 \text { months to } \\
3 \text { months }\end{array}$ & $\begin{array}{l}2 \operatorname{RCTs}^{52,54} \\
n=228\end{array}$ & $\begin{array}{l}\text { Inconclusive } \\
\text { Percent discharged home: ONS: } \\
19.9 \text { (2.0); CG: } 20.2(2.4) \text { ( } n=80 ; \\
\text { 1 RCT); no difference } \\
\text { Proportion of patients discharged } \\
\text { to residential facility favors } \\
\text { intervention ( } n=148 ; 1 \text { RCT) }\end{array}$ & High & Inconsistent & $\begin{array}{l}\text { Imprecise (no } \\
\text { information on } \\
95 \% \text { Cls of } \\
\text { one of two } \\
\text { estimates) }\end{array}$ & Direct & Insufficient \\
\hline
\end{tabular}

*1-month, 3-month, and 6-month data extracted from survival analysis curves using a Web-based numerical data extraction tool (WebPlotDigitizer v 4.4) to estimate graphical data. 12-month mortality data matched text data, although, the survival analysis plot portrayed different results than described in text (Treatment $10.2 \%$ vs Control Group $36.1 \%$ ). Data reported are from the survival analysis.

Note: We used the following 95\% CI bounds recognized by GRADE and others to determine evidence of no difference: Summary estimates using ratio statistics: Lower CI 0.80 to Upper CI 1.25; Summary estimates using standardized mean difference: Lower CI -0.2 to Upper CI 0.2; Summary estimates using raw mean difference: Depends on measure or instrument; default is $20 \%$ difference on each side

$\mathrm{CG}=$ control group; $\mathrm{CI}=$ confidence interval; EQ-5D = EuroQol 5 dimension; $\mathrm{NR}=$ not reported; ONS = oral nutrition supplement; PCS = physical component summary;

$\mathrm{Q} 1$ = first quartile; $\mathrm{Q} 3$ = third quartile; $\mathrm{QoL}=$ quality of life; $\mathrm{RCT}=$ randomized controlled trial; $\mathrm{RR}=$ relative risk; SF-36 = 36 item short form health survey;

$\mathrm{SMD}=$ standardized mean difference; $\mathrm{SOE}=$ stenght of evidence; $\mathrm{Tx}=$ treatment $\mathrm{WMD}=$ weighted mean difference 


\section{Discussion}

In fiscal year 2020, Congress requested that the Agency for Healthcare Research and Quality (AHRQ) convene a panel of experts charged with developing quality measures for malnutritionrelated hospital readmissions. At AHRQ's request, we conducted a systematic review to inform the deliberations and recommendations of this panel. We focused our Key Questions (KQs) on the following: 1) reviewing the association between malnutrition and clinical outcomes, 2) evaluating the effectiveness of screening and/or diagnostic assessment of malnutrition on clinical outcomes, and 3) assessing the effectiveness of hospital-initiated interventions to treat patients diagnosed with malnutrition

\section{Findings in Relation to Decisional Dilemma(s)}

\section{Malnutrition and Clinical Outcomes}

Our review confirmed that malnutrition (defined using commonly available measurement tools) is associated with poor clinical outcomes (KQ 1). Existing systematic reviews found low strength of evidence (SOE) that malnutrition may be associated with increased hospital mortality. This association was observed across patients hospitalized for acute medical conditions, heart failure, and cirrhosis. Moderate SOE also showed that malnutrition (diagnosed using Subjective Global Assessment [SGA]) was independently associated with prolonged hospital length of stay among patients hospitalized with acute medical conditions or cancer. Evidence for other measurement tools used to identify malnutrition (Nutritional Risk Screening2002 [NRS-2002], Malnutrition Universal Screening Tool [MUST], Mini Nutritional AssessemtShort Form [MNA-SF], Short Nutritional Assessment Questionnaire [SNAQ], and Prognosis Inflammatory and Nutritional Index PINI) was largely insufficient to determine if malnutrition was associated with clinical outcomes.

\section{Malnutrition Screening and Interventions}

Given the potentially detrimental effects of malnutrition, identifying patients who could benefit from further assessment or an intervention for malnutrition is crucial. Currently, U. S. hospitals are mandated to screen all hospitalized patients for malnutrition within 24 hours of admission. However, at present, whether such screening improves clinical outcomes remains unknown. Thus, KQ 2 aimed to assess if malnutrition screening and/or diagnostic assessment prompts actions that change either nutritional stores or key clinical outcomes, such as mortality, length of stay, readmissions, or hospital acquired complications.

As previously noted, we identified no studies addressing this question. Studies we excluded either lacked an unexposed group (i.e., no screening group) or used an inappropriate comparator (i.e., did not use an acceptable reference standard as a comparator). These studies were inadequate because without an unexposed group or appropriate control it was not possible to determine if any observed effects are attributable to screening or at least to a particular screening or diagnostic assessment tool. Additionally, retrospective studies or studies using a historical control group were not included given their high potential for biases related to confounding effects and patient selection.

While no studies addressed KQ 2, we did identify randomized controlled trials (RCTs) indicating that some malnutrition interventions improve clinical outcomes among patients who are diagnosed with malnutrition or screened at risk using commonly available measurement tools 
(KQ 3). Specifically, we found moderate SOE from 11 RCTs that hospital initiated specialized nutrition care and increased protein/calorie provision likely reduce mortality compared to usual care. We also found that these interventions may improve quality of life (SOE: Low). The evidence, however, was either insufficient or showed no difference between groups for other outcomes: length of stay, readmissions, activities of daily living, discharge disposition, hospital acquired conditions, or adverse events. Of course, the benefits of hospital-initiated interventions on hospitalization associated outcomes such as length of stay, discharge disposition, and hospital associated complication may be limited as weeks to months are often required to resolve the deleterious impact of malnutrition.

\section{Findings in Relation to What Is Known}

Our review underscores many known limitations of research on nutritional measurement tools: these problems include varied definitions of malnutrition in the literature, lack of validated tools, and lack of an accepted reference standard.

As anticipated, we found malnutrition studies have employed a wide range of definitions for malnutrition. For example, one prior systematic review (SR) of nutrition-focused interventions for hospitalized patients by Feinberg et al. (2017) ${ }^{59}$ included 244 RCTs. However, over 40 percent of these trials (105 of 244) did not use a commonly available measurement tool to confirm the diagnosis of malnutrition, instead relying on presence of severe disease, weight loss, body mass index (BMI) (or other biomarkers), or clinical opinion to define a malnourished population. Thus, we focused on more recent literature, our SR captured studies published from 2000 onwards to try to capture literature better aligned with current recommendations for screening and diagnosis established by the Global Leadership Initiative on Malnutrition (GLIM) taskforce (2019). However, we found that relatively few studies have used criteria aligned with the GLIM criteria to define malnourished patients. Since the GLIM consensus criteria were established relatively recently, it is likely that not enough time has passed for studies to employ these criteria in trials. The few studies identified in our searches that focused on validation of GLIM criteria were excluded for KQ 2 as they did not meet study design criteria for our report (i.e., they were uncontrolled trials).

Even when malnourished patients are identified using validated tools, inconsistent agreement and reliability may be problematic, particularly given the large number of different instruments currently in use. Skipper et al. (2012) performed an SR to assess the validity of available screening tools used to identify patients at-risk of malnutrition. ${ }^{61}$ Authors found that available tools only achieved moderate- rather than high-level validity, agreement, and reliability, and that there were large variations in these measures for all tools. Authors attributed the large range in validity and reliability to researchers using different reference standards to validate tools and suggested that use of a single reference standard would narrow the ranges of reliability and validity. Differences in validity of measurement tools likely contributed to variations observed in the SOE for the association of malnutrition with poor clinical outcomes. Use of unvalidated instruments as reference standards was also a common reason for excluding studies from KQ 2.

Regarding hospital-initiated interventions, our findings aligned with prior reviews for the important outcome of mortality, despite large differences in how malnutrition was defined. Two previous systematic reviews, one by Feinberg et al. $(2017)^{60}$ and the other by Gomes et al. (2019), ${ }^{59}$ examined the efficacy and safety of various nutrition-focused interventions used to treat hospitalized patients. These reviews included studies published prior to 2000 and included trials of patients designated malnourished based on biometrics (e.g., BMI, weight loss, serum 
albumin level), severity of disease (e.g., any intensive care unit admission) or clinical judgment. Like our review, these reviews also found that nutrition-focused interventions decreased mortality.

\section{Applicability}

As noted, in consultation with our Technical Expert Panel (TEP) and to align with current consensus recommendations for screening and diagnosis, our review was limited to studies that confirmed a diagnosis of malnutrition using commonly available measurement tools. Thus, our findings regarding clinical outcomes and interventions are applicable to other patients with malnutrition determined in this way. However, conversely, it remains unclear to what extent these findings are generalizable across malnourished populations not identified in this way.

Using these criteria to define malnutrition also indirectly led to exclusion of studies assessing more invasive nutritional interventions for hospitalized patients such as parenteral nutrition or enteral nutrition. Studies assessing these interventions initiated treatment based on severity of illness, clinical judgement, or surrogate markers of malnutrition, such as blood serum markers and other biometrics. Thus, our findings only extend to two types of hospital-initiated malnutrition interventions, specialized nutrition care (i.e., consultation with a nutrition specialist and individualized goals) and oral protein/calorie supplementation. Also, while included studies enrolled older patients and patients with a range of underlying clinical conditions, we did not have enough studies to conduct subgroup analysis to determine if the effects of treatment differed depending on patient characteristics.

\section{Limitations and Suggestions for Future Research}

\section{Defining Malnutrition}

A key challenge for assessing studies of malnutrition is determining how malnutrition should be defined. Although many studies have defined malnutrition using biomarkers (e.g. BMI, weight loss, serum albumin levels) experts have expressed concern that these measures are not reliable indices of malnutrition by themselves. ${ }^{49}$ For instance, serum albumin levels often fluctuate in response to physiological stress and other factors unrelated to a patient's nutritional status. ${ }^{62}$ Similarly, metrics such as BMI fail to account for variations related to gender, age, race, or body type. ${ }^{63}$ Other studies have used severity of disease (e.g. any intensive care admission) as a proxy for or criterion to intervene on malnutrition often without formal diagnostic assessment. Therefore, there is wide variability in how malnutrition has been identified and studied.

The wide range of definitions (and measurement tools) has created challenges for clinical practice and malnutrition research. To address this problem, in 2019, the GLIM taskforce recommended a two-step approach to identifying malnutrition that involves 1) screening for malnutrition using a valid tool, and 2) performing a formal diagnostic assessment for those who screen positive. ${ }^{16} \mathrm{~A}$ formal diagnosis of malnutrition according to GLIM recommendations requires patients to have at least one etiologic factor (reduced food intake, hypercatabolic burden of disease) and one phenotypical factor (non-volitional weight loss, low BMI, low skeletal muscle mass). However, GLIM recommendations have yet to be clinically validated or widely applied in clinical practice or research settings. ${ }^{19}$ 
Finally, defining malnutrition requires the validation of measurement tools against a gold standard. However, through discussions with our TEP, we recognized that there currently is no universally agreed upon gold standard for malnutrition assessment and measurement. For the purposes of this report, we selected, with input from our TEP and subject matter experts, imaging modalities to quantify and evalute body composition (i.e., muscle and adipose tissues) as the gold standard and SGA as a semi-gold standard for classifying malnutrition. However, use of imaging specifically to assess malnutrition is infrequent and has important limitations, including cost, radiation exposure, and need for serial studies. In addition, at present, none of the current assessment tools (malnutrition measurement tools, the GLIM framework, or imaging modalities) capture micronutrient deficiencies. Development of an accepted gold standard for defining malnutrition is key to supporting future clinical care and research.

\section{Effectiveness of Measurement Tools}

This review highlights several important knowledge gaps in the current literature that future research needs to address. One is the relatively small number of studies that used available measurement tools to identify malnutrition. As noted, many studies identified malnutrition based only on biometrical measures, such as serum albumin levels, BMI, and weight, despite consensus that albumin and BMI should not be used to define malnutrition in practice or research. Thus, future studies assessing the impact of malnutrition on outcomes or evaluating malnutritionfocused interventions should use known tools to establish malnutrition status. Future studies would also benefit from stratifying patients by age, gender, and frailty.

Second is the absence of studies meeting inclusion criteria that address KQ 2. The absence of studies addressing the clinical utility (effectiveness) of measurement tools for nutrition screening and diagnostic assessment (KQ 2) does not necessarily imply that these tools are ineffective. However, it reveals the need for appropriately designed studies to better understand the downstream consequences of nutrition screening, including subsequent diagnostic assessment, management, and clinical outcomes is extremely important given that hospitals are mandated to provide nutrition screening for all hospitalized patients within 24 hours of admission.

One way to indirectly address this is to determine if one measure-SGA, imaging modalities, or the new GLIM criteria - better captures clinically important malnutrition. To assess which measure is more effective, one could envision a multi-arm clinical trial that compares multiple tools and techniques. For example, a study could screen hospitalized patients as mandated by the Joint Commission, and further assess at-risk patients with each of these diagnostic assessment tools; results of one diagnostic assessment tool could then be used to randomize patients (i.e., those diagnosed with malnutrition) to nutritional interventions. This would provide better understanding of the clinical course for patients who test negative by various diagnostic assessments and provide insights on potential harms of using specific tools. Furthermore, given significant overlap in the variables utilized in the tools, future research could also support identification of which variables have the greatest impact on sensitivity and specificity in prospective clinical studies.

\section{Malnutrition Interventions}

Finally, the studies addressing the efficacy of malnutrition-focused interventions were limited to studies of specialized nutrition care (consultation with a dietitian to set goals for protein and caloric intake) or increased protein/calorie supplementation. These studies had 
several shortcomings, including high risk of bias and poor reporting of treatment-related adverse events. Most studies were rated as high risk of bias or had some concerns with outcome-level risks of bias. In most cases, studies failed to report adequate randomization, had unknown allocation concealment, and were unclear if all pre-specified outcomes were reported. Some studies also had high dropout rates ( $>20$ percent) and did not blind outcome assessors. These limitations, along with inconsistencies in the findings for some outcomes and lack of precision for others, downgraded the overall strength of the evidence to low or insufficient for most outcomes. These studies either did not report on harms of treatment or reported on harms in a manner that did not allow us to synthesize the data. Thus, our review does not capture harms associated with the assessed treatments. Specifically, future studies should randomize patients diagnosed with malnutrition (i.e., using a diagnostic assessment tool) to different interventions, such as parenteral and enteral nutrition, conduct subgroup analyses to assess the benefits of nutritional intervention in subpopulations, and document harms associated with treatment.

\section{Implications for Clinical Practice, Education, Research, or Health Policy}

Our SR was intended to inform the deliberations of a congressional panel charged with developing quality measures for malnutrition-related hospital readmissions. Such measures would potentially support assigning accountability for the assessment and treatment of malnutrition in hospitalized adults, with an emphasis on the needs of older frail adults. Although our review confirmed that malnutrition is associated with poor outcomes and that specific interventions may be beneficial, it also highlights many challenges of drawing conclusions from this evidence base, starting with fundamental questions regarding how malnutrition should be defined and measured.

Variations in how malnutrition is defined and measured pose a challenge for hospitals seeking to standardize processes for screening, further assessing, and documenting diagnosis of malnutrition. As previously mentioned in the Background section of this review, national survey data indicate that only 38 percent of hospital professionals report using a recognized tool to screen for malnutrition, and only 23 percent report using one for diagnostic assessment in those who screen positive. ${ }^{7}$ Another challenge to standardizing these processes is ensuring that the definition or criteria for malnutrition reflected within available measurement tools are consistent with current documentation and coding requirements for malnutrition. For instance, the International Classification of Diseases, 10th edition (ICD-10) coding system has different codes for varying levels of severity of malnutrition that are not always aligned with how malnutrition is defined using GLIM or American Society for Parenteral and Enteral Nutrition (ASPEN) criteria. $^{64,65}$

Further research such as a multi-arm trial that randomizes patients to different measurement tools would allow researchers and practitioners to further understand the clinical utility of each tool, including downstream potential benefits and harms. Similarly, studies that acknowledge the notable overlap in variables utilized amongst these measurement tools may help identify which variables have the greatest sensitivity and specificity, impact on clinical outcomes, and lead to development of a comprehensive tool. For example, some tools may benefit from removing outdated variables, such as BMI. Such research could support the complex and evolving task of disentangling disease (i.e., severity of illness) and nutritional status.

As for malnutrition interventions, no studies were identified specific to the effect of parenteral or enteral nutrition in patients diagnosed with malnutrition, representing a significant 
evidence gap in malnourished hospitalized patients. Further research in this area is essential to determine which malnourished patient populations benefit from specific types of interventions. Key guidance for hospitals is needed on how to standardize processes for screening, diagnosing and documenting malnutrition in order to inform development of quality measures and to improve patient outcomes. 


\section{References}

1. Somanchi M, Tao X, Mullin GE. The facilitated early enteral and dietary management effectiveness trial in hospitalized patients with malnutrition. JPEN J Parenter Enteral Nutr. 2011 Mar;35(2):209-16. http://dx.doi.org/10.1177/01486071103922 34. PMID: 21378250.

2. Definitions: malnutrition. [internet]. Silver Spring (MD): American Society for Parenteral and Enteral Nutrition (ASPEN); [accessed 2020 Aug 14]. [2 p]. https://www.nutritioncare.org/Guidelines and Clinical Resources/Toolkits/Malnu trition Toolkit/Definitions/.

3. What is malnutrition? . [internet]. Geneva, Switzerland: World Health Organization; 2020 Apr 15 [accessed 2021 Jul 26]. [3 p]. https://www.who.int/news-room/q-adetail/malnutrition.

4. Corkins MR, Guenter P, DiMaria-Ghalili RA, et al. Malnutrition diagnoses in hospitalized patients: United States, 2010. JPEN J Parenter Enteral Nutr. 2014 Feb;38(2):186-95.

http://dx.doi.org/10.1177/01486071135121 54. PMID: 24247093.

5. Weiss AJ, Fingar KR, Barrett ML, et al. Characteristics of hospital stays involving malnutrition, 2013: HCUP Statistical Brief \#210. In: Healthcare Cost and Utilization Project (HCUP) Statistical Briefs. Rockville (MD): Agency for Healthcare Research and Quality; 2006 Feb-; 2016 Sep. p. 21.

http://www.hcupus.ahrq.gov/reports/statbriefs/sb210Malnutrition-Hospital-Stays-2013.pdf. PMID: 27854406.

6. Barrett ML, Bailey MK, Owens PL. Nonmaternal and non-neonatal inpatient stays in the United States involving malnutrition.

Rockville (MD): Healthcare Cost and Utilization Project (HCUP). Agency for Healthcare Research and Quality (AHRQ); 2016. 29 p. https://www.hcupus.ahrq.gov/reports.jsp.
7. Patel V, Romano M, Corkins MR, et al. Nutrition screening and assessment in hospitalized patients: a survey of current practice in the United States. Nutr Clin Pract. 2014 Aug;29(4):483-90. http://dx.doi.org/10.1177/08845336145354 46. PMID: 24993585.

8. Mogensen KM, Bouma S, Haney A, et al. Hospital nutrition assessment practice 2016 survey. Nutr Clin Pract. 2018 Oct;33(5):711-7. http://dx.doi.org/10.1002/ncp.10179. PMID: 30088829.

9. Theilla M, Rattanachaiwong S, Kagan I, et al. Validation of GLIM malnutrition criteria for diagnosis of malnutrition in ICU patients: an observational study. Clin Nutr. 2020 Dec 29;S0261-5614(20):30696-8. http://dx.doi.org/10.1016/j.clnu.2020.12.02 1. PMID: 33413910.

10. List of measures under consideration for December 21, 2020. Baltimore (MD): Centers for Medicare and Medicaid Services (CMS); 2020. 85 p. https://www.cms.gov/files/document/meas ures-under-consideration-list-2020report.pdf.

11. McClave SA, Taylor BE, Martindale RG, et al. Guidelines for the provision and assessment of nutrition support therapy in the adult critically ill patient: Society of Critical Care Medicine (SCCM) and American Society for Parenteral and Enteral Nutrition (A.S.P.E.N.). JPEN J Parenter Enteral Nutr. 2016 Feb;40(2):159-211. http://dx.doi.org/10.1177/01486071156218 63. PMID: 26773077.

12. Charney P. Nutrition screening vs nutrition assessment: how do they differ? Nutr Clin Pract. 2008 Aug-Sep;23(4):366-72. http://dx.doi.org/10.1177/08845336083211 31. PMID: 18682587.

13. Correia MITD. Nutrition screening vs nutrition assessment: what's the difference? Nutr Clin Pract. 2018 Feb;33(1):62-72. http://dx.doi.org/10.1177/08845336177196 69. PMID: 28727954. 
14. Mogensen KM, Malone A, Becker P, et al. Academy of Nutrition and Dietetics/American Society for Parenteral and Enteral Nutrition consensus malnutrition characteristics: usability and association with outcomes. Nutr Clin Pract. 2019 Oct;34(5):657-65.

http://dx.doi.org/10.1002/ncp.10310. PMID: 31074906.

15. White JV, Guenter P, Jensen G, et al. Consensus statement: Academy of Nutrition and Dietetics and American Society for Parenteral and Enteral Nutrition: characteristics recommended for the identification and documentation of adult malnutrition (undernutrition). JPEN J Parenter Enteral Nutr. 2012 May;36(3):27583.

http://dx.doi.org/10.1177/01486071124402 85. PMID: 22535923.

16. Cederholm T, Jensen GL, Correia MITD, et al. GLIM criteria for the diagnosis of malnutrition - a consensus report from the global clinical nutrition community. Clin Nutr. 2019 Feb;38(1):1-9. http://dx.doi.org/10.1016/j.clnu.2018.08.00 2. PMID: 30181091.

17. Mordarski B. Nutrition-focused physical exam hands-on training workshop. J Acad Nutr Diet. 2016 May;116(5):868-9. http://dx.doi.org/10.1016/i.jand.2016.03.0 04. PMID: 27126160.

18. Mueller C, Compher C, Druyan ME. A.S.P.E.N. clinical guidelines: nutrition screening, assessment, and intervention in adults. JPEN J Parenter Enteral Nutr. 2011 Jan;35(1):16-24. PMID: 21224430.

19. Keller H, de van der Schueren MAE, Jensen GL, et al. Global Leadership Initiative on Malnutrition (GLIM): guidance on validation of the operational criteria for the diagnosis of protein-energy malnutrition in adults. JPEN J Parenter Enteral Nutr. 2020 Aug;44(6):992-1003. http://dx.doi.org/10.1002/jpen.1806. PMID: 32529700

20. McClave SA, DiBaise JK, Mullin GE, et al. ACG clinical guideline: nutrition therapy in the adult hospitalized patient. Am J Gastroenterol. 2016 Mar;111(3):315-34; quiz 335 .

http://dx.doi.org/10.1038/ajg.2016.28. PMID: 26952578.
21. Owens D, Lohr K, Atkins D, et al. Grading the strength of a body of evidence when comparing medical interventions. In: Agency for Healthcare Research and Quality (AHRQ). Methods guide for comparative effectiveness reviews. Rockville (MD):

Agency for Healthcare Research and Quality (AHRQ); 2009 Jul.

https://effectivehealthcare.ahrq.gov/prod ucts/methods-guidance-gradingstrength/methods.

22. Rohatgi A. WebPlotDigitizer. [software] Ver. 4.4. Pacifica (CA): 2020 Nov. https://automeris.io/WebPlotDigitizer/.

23. Cochrane Methods Network. Risk of bias 2 (RoB2) tool. [internet]. London (England): The Cochrane Collaboration; [accessed 2021 Feb 08]. [3 p]. https://methods.cochrane.org/risk-bias-2.

24. Sterne JA, Hernán MA, Reeves BC, et al. ROBINS-I: a tool for assessing risk of bias in non-randomised studies of interventions. BMJ. 2016 Oct 12;355:i4919. http://dx.doi.org/10.1136/bmj.i4919. PMID: 27733354

25. Whiting P, Savovic J, Higgins J, et al. ROBIS: tool to assess risk of bias in systematic reviews. Guidance on how to use ROBIS. Bristol (England): Bristol Medical School, Population Health Sciences, University of Bristol; 2015. 39 p. http://www.bristol.ac.uk/medialibrary/sites/social-communitymedicine/robis/robisguidancedocument.p df.

26. McGrath S, Zhao X, Steele R, et al. Estimating the sample mean and standard deviation from commonly reported quantiles in meta-analysis. Stat Methods Med Res. 2020;29(9):2520-37. http://dx.doi.org/10.1177/09622802198890 80. PMID: 32292115.

27. Hartung J, Knapp G. A refined method for the meta-analysis of controlled clinical trials with binary outcome. Stat Med. 2001 Dec 30;20(24):3875-89.

28. Cornell JE, Mulrow CD, Localio R, et al. Random-effects meta-analysis of inconsistent effects: a time for change. Ann Intern Med. 2014 Feb 18;160(4):267-71. http://dx.doi.org/10.7326/M13-2886. PMID: 24727843 
29. Fisher D. admetan. A new, comprehensive meta-analysis command. Presented at 2018 London Stata Conference. [slide presentation]. London (England): University College London, MRC Clinical Trials Unit; 2018 Sep 6. 19 p.

30. Dijkink S, Meier K, Krijnen P, et al. Malnutrition and its effects in severely injured trauma patients. Eur J Trauma Emerg Surg. 2020 Oct;46(5):993-1004. Epub 2020 Jan 23.

http://dx.doi.org/10.1007/s00068-02001304-5. PMID: 31974669.

31. Lew CCH, Yandell R, Fraser RJL, et al. Association between malnutrition and clinical outcomes in the intensive care unit: a systematic review. J Parenter Enteral Nutr. 2017 Jul;41(5):744-58.

http://dx.doi.org/10.1177/01486071156256 38. PMID: 26838530.

32. Lin H, Zhang H, Lin Z, et al. Review of nutritional screening and assessment tools and clinical outcomes in heart failure. Heart Fail Rev. 2016 Sep;21(5):549-65. http://dx.doi.org/10.1007/s10741-0169540-0. PMID: 26920682.

33. Gupta D, Vashi PG, Lammersfeld CA, et al. Role of nutritional status in predicting the length of stay in cancer: a systematic review of the epidemiological literature. Ann Nutr Metab. 2011 Dec;59(2):96-106. http://dx.doi.org/10.1159/000332914. PMID: 22142719.

34. Muscaritoli M, Krznaric Z, Singer P, et al. Effectiveness and efficacy of nutritional therapy: a systematic review following Cochrane methodology. Clin Nutr. 2017 Aug;36(4):939-57. http://dx.doi.org/10.1016/j.clnu.2016.06.02 2. PMID: 27448948.

35. Ney M, Li S, Vandermeer B, et al. Systematic review with meta-analysis: nutritional screening and assessment tools in cirrhosis. Liver Int. 2020 Mar;40(3):664-73. http://dx.doi.org/10.1111/liv.14269. PMID: 31571398.

36. Rypkema G, Adang E, Dicke H, et al. Costeffectiveness of an interdisciplinary intervention in geriatric inpatients to prevent malnutrition. J Nutr Health Aging. 2004;8(2):122-7. PMID: 14978608.
37. Tapia MJ, Ocón J, Cabrejas-Gómez C, et al. Nutrition-related risk indexes and long-term mortality in noncritically ill inpatients who receive total parenteral nutrition (prospective multicenter study). Clin Nutr. 2015 Oct;34(5):962-7.

http://dx.doi.org/10.1016/j.clnu.2014.10.00 8. PMID: 25466952 .

38. Thomas J, Kaambwa B, Delaney C, et al. An evaluation of the validity of nutrition screening and assessment tools in patients admitted to a vascular surgery unit. $\mathrm{Br} \mathbf{J}$ Nutr. 2019 Sep 28;122(6):689-97. http://dx.doi.org/10.1017/S0007114519001 442. PMID: 31256768.

39. Martins CP, Correia JR, Do Amaral TF. Undernutrition risk screening and length of stay of hospitalized elderly. J Nutr Elder. 2006 Dec 13;25(2):5-21. http://dx.doi.org/10.1300/J052v25n02 02 . PMID: 17182464.

40. Kruizenga HM, Van Tulder MW, Seidell JC, et al. Effectiveness and costeffectiveness of early screening and treatment of malnourished patients. Am J Clin Nutr. 2005;82(5):1082-9. PMID: 16280442.

41. Holyday M, Daniells S, Bare M, et al. Malnutrition screening and early nutrition intervention in hospitalised patients in acute aged care: a randomised controlled trial. $\mathrm{J}$ Nutr Health Aging. 2012 Jun;16(6):562-8. http://dx.doi.org/10.1007/s12603-0120022-3. PMID: 22659998.

42. Johansen N, Kondrup J, Plum LM, et al. Effect of nutritional support on clinical outcome in patients at nutritional risk. Clin Nutr. 2004 Aug;23(4):539-50. PMID: 15297090 .

43. Lomivorotov VV, Efremov SM, Boboshko VA, et al. Evaluation of nutritional screening tools among patients scheduled for heart valve surgery. J Heart Valve Dis. 2013 Mar;22(2):239-47. PMID: 23798215.

44. Velasco C, García E, Rodríguez V, et al. Comparison of four nutritional screening tools to detect nutritional risk in hospitalized patients: a multicentre study. Eur J Clin Nutr. 2011 Feb;65(2):269-74. http://dx.doi.org/10.1038/ejen.2010.243. PMID: 21081958. 
45. Zhang Z, Wan Z, Zhu Y, et al. Prevalence of malnutrition comparing NRS2002, MUST, and PG-SGA with the GLIM criteria in adults with cancer: a multi-center study. Nutrition. 2021 Mar;83:111072. http://dx.doi.org/10.1016/j.nut.2020.11107 2. PMID: 33360034.

46. Kroc L, Fife E, Piechocka-Wochniak E, et al. Comparison of nutrition risk screening 2002 and subjective global assessment form as short nutrition assessment tools in older hospitalized adults. Nutrients. 2021 Jan;13(1):1-10. http://dx.doi.org/10.3390/nu13010225. PMID: 33466724.

47. Becker L, Volkert D, Christian Sieber C, et al. Predictability of a modified mininutritional- assessment version on six-month and one-year mortality in hospitalized geriatric patients: a comparative analysis. Sci Rep. 2019 Jun 21;9(1):9064. http://dx.doi.org/10.1038/s41598-01945452-0. PMID: 31227778.

48. Omidvari AH, Vali Y, Murray SM, et al. Nutritional screening for improving professional practice for patient outcomes in hospital and primary care settings. Cochrane Database Syst Rev. 2013 Jun 6;(6):CD005539. http://dx.doi.org/10.1002/14651858.CD00 5539.pub2. PMID: 23744516.

49. Olveira G, Tapia MJ, Ocón J, et al. Study Group of Hyperglycemia in Parenteral Nutrition; Nutrition Area of the Spanish Society of Endocrinology and Nutrition (SEEN). The subjective global assessment predicts in-hospital mortality better than other nutrition-related risk indexes in noncritically ill inpatients who receive total parenteral nutrition in Spain (prospective multicenter study). J Acad Nutr Diet. 2013 Sep;113(9):1209-18.

http://dx.doi.org/10.1016/j.jand.2013.05.0 27. PMID: 23972272.

50. Bonilla-Palomas JL, Gámez-López AL, Castillo-Domînguez JC, et al. Nutritional intervention in malnourished hospitalized patients with heart failure. Arch Med Res. 2016 Oct;47(7):535-40.

http://dx.doi.org/10.1016/j.arcmed.2016.1 1.005. PMID: 28262195.
51. Ha L, Hauge T, Spenning AB, et al. Individual, nutritional support prevents undernutrition, increases muscle strength and improves QoL among elderly at nutritional risk hospitalized for acute stroke: a randomized, controlled trial. Clin Nutr. 2010 Oct;29(5):567-73. http://dx.doi.org/10.1016/j.clnu.2010.01.01 1. PMID: 20176418.

52. Sharma Y, Thompson CH, Kaambwa B, et al. Investigation of the benefits of early malnutrition screening with telehealth follow up in elderly acute medical admissions. QJM. 2017 Oct;110(10):639-47. http://dx.doi.org/10.1093/qjmed/hcx095. PMID: 28472530.

53. Deutz NE, Matheson EM, Matarese LE, et al. Readmission and mortality in malnourished, older, hospitalized adults treated with a specialized oral nutritional supplement: a randomized clinical trial. Clin Nutr. 2016 Feb;35(1):18-26.

http://dx.doi.org/10.1016/j.clnu.2015.12.01 o. PMID: 26797412.

54. Gazzotti C, Arnaud-Battandier F, Parello M, et al. Prevention of malnutrition in older people during and after hospitalisation: results from a randomised controlled clinical trial. Age Ageing. 2003 May;32(3):321-5. http://dx.doi.org/10.1093/ageing/32.3.321. PMID: 12720620.

55. Schuetz P, Fehr R, Baechli V, et al. Individualised nutritional support in medical inpatients at nutritional risk: a randomised clinical trial. Lancet. 2019 Jun 8;393(10188):2312-21. http://dx.doi.org/10.1016/S01406736(18)32776-4. PMID: 31030981.

56. Starke J, Schneider H, Alteheld B, et al. Short-term individual nutritional care as part of routine clinical setting improves outcome and quality of life in malnourished medical patients. Clin Nutr. 2011 Apr;30(2):194201.

http://dx.doi.org/10.1016/j.clnu.2010.07.02 1. PMID: 20937544.

57. Rüfenacht U, Rühlin M, Wegmann M, et al. Nutritional counseling improves quality of life and nutrient intake in hospitalized undernourished patients. Nutrition. 2010 Jan;26(1):53-60. http://dx.doi.org/10.1016/j.nut.2009.04.01 8. PMID: 19695832 . 
58. Munk T, Beck AM, Holst M, et al. Positive effect of protein-supplemented hospital food on protein intake in patients at nutritional risk: a randomised controlled trial. J Hum Nutr Diet. 2014 Apr;27(2):122-32. http://dx.doi.org/10.1111/jhn.12210. PMID: 24479388.

59. Gomes F, Baumgartner A, Bounoure L, et al. Association of nutritional support with clinical outcomes among medical inpatients who are malnourished or at nutritional risk: an updated systematic review and metaanalysis. JAMA Netw Open. 2019 Nov;2(11):e1915138. http://dx.doi.org/10.1001/jamanetworkop en.2019.15138. PMID: 31747030.

60. Feinberg J, Nielsen EE, Korang SK, et al. Nutrition support in hospitalised adults at nutritional risk. Cochrane Database Syst Rev. 2017 May 19;(5):CD011598. http://dx.doi.org/10.1002/14651858.CD01 1598.pub2. PMID: 28524930.

61. Skipper A, Ferguson M, Thompson K, et al. Nutrition screening tools: an analysis of the evidence. JPEN J Parenter Enteral Nutr. 2012 May;36(3):292-8. http://dx.doi.org/10.1177/01486071114140 23. PMID: 22045723.
62. Bharadwaj S, Ginoya S, Tandon P, et al. Malnutrition: laboratory markers vs nutritional assessment. Gastroenterol Rep (Oxf). 2016 Nov;4(4):272-80. Epub 2016 May 11.

http://dx.doi.org/10.1093/gastro/gow013. PMID: 27174435.

63. Barone M. Is the use of the BMI alone sufficient to diagnose malnutrition in both male and female adults? Clin Nutr. 2018 Oct;37(5):1771.

http://dx.doi.org/10.1016/j.clnu.2018.07.00 3. PMID: 30055866.

64. Q\&A: Documentation and ICD-10-CM coding for severe malnutrition. CDI Strategies. 2019 Sep 12;13(38)

https://acdis.org/articles/qadocumentation-and-icd-10-cm-codingsevere-malnutrition.

65. A step-by-step guide to implementing a malnutrition coding program for adult inpatients. Support Line. 2017 Jun;39(3):29. http://oknutrition.org/images/A StepBy-

Step Guide to Implementing a Malnutr ition Coding Program for Adult Patien ts.pdf. 


\section{Appendix A. Search Strategy}

\section{Search Details and Data Sources}

The search strategies for the individual databases were developed, executed, and documented by an experienced EPC librarian and were peer reviewed by an experienced methodologist. We applied the following limits or filters to the database searches:

- Date. The literature search covered January 2000 to June 2021.

- Language. Publications were excluded if they were written in a language other than English.

- Publication status. We searched for published studies.

- Human or organism. The search was limited to human studies.

- Study design. The search was restricted to randomized and non-randomized studies related to Key Question 2 and randomized studies, systematic reviews, and meta-analyses for Key Questions 1 and 3.

We conducted a comprehensive literature search over the course of several months spanning October 2020 - December 2020 (updated March 2021 and again June 2021). Key Questions were searched separately on different dates and there were several searches that included additional terms and expanded concepts. Update searches covering all Key Questions, additional terms, and expanded concepts in all databases were run in March 2021 and again in June 2021. We searched the following databases: EMBASE \& MEDLINE (Embase.com) (2000 - 2020) Dates searched October 7, 2020, October 19, 2020, November 20, 2020, November 23, 2020, and December 11, 2020; PubMed in process citations (2000 - 2020) Date searched November 5, 2020 .

\section{Search Strategy}

This section describes the search strategies.

\section{$\underline{\text { Embase }}$}

1 'cachexia'/de OR 'malnutrition'/de OR cachexi* OR malnourish* OR malnutrition OR nutrition:ti OR underfed OR undernourish* OR undernutrition

2 (malnutrition OR nutrition*) NEAR/3 risk

3 'nutrition* status' OR 'nutrition* store*'

4 'aged hospital patient'/de OR 'hospital patient'/de OR hospitalis* OR hospitaliz* OR icu OR inpatient* OR 'intensive care unit' OR ((hospital* NEAR/5 patient*):ab,ti) OR (hospital* NEAR/5 (admit* OR admission*))

5 (\#1 OR \#2 OR \#3) AND \#4

6 \#5 AND ([cochrane review]/lim OR [systematic review]/lim OR [meta analysis]/lim OR cochrane OR 'meta analysis' OR 'meta analyses' OR metaanlysis OR metaanalyses OR search* OR systematic:ti)

7 'nutritional screening'/exp/mj OR 'short nutritional assessment questionnaire'/exp OR 'screening'/exp OR screen* OR 'Malnutrition Screening Tool' OR MST OR 'Malnutrition Universal Screening Tool' OR MUST OR 'Nutritional Risk Index' OR NRI OR 'Nutrition Risk in Critically Ill' OR NUTRIC OR (nutrition*:ti AND (assess*:ti OR screen*:ti)) OR 'nutrition 
risk screening 2002' OR 'nrs2002' OR 'nrs-2002' OR 'short nutritional assessment questionnaire' OR snaq

8 'nutritional assessment'/exp/mj OR 'diagnostic assessment' OR 'subjective global assessment' OR sga OR 'patient generated subjective global assessment' OR 'ps-sga' OR 'mini nutritional assessment' OR mna OR 'and-aspen mcc' OR 'global leadership initiative on malnutrition' OR glim OR 'malnutrition consensus criteria' OR (('academy of nutrition and dietetics' OR 'and') AND ('american society for parenteral and enteral nutrition' OR aspen OR 'a.s.p.e.n.') AND (consensus OR mcc))

9 \#5 AND (\#7 OR \#8)

10 \#9 AND ([cochrane review]/lim OR [systematic review]/lim OR [meta analysis]/lim OR cochrane OR 'meta analysis' OR 'meta analyses' OR metaanlysis OR metaanalyses OR search* OR systematic:ti)

11 \#9 AND ('randomized controlled trial'/de OR 'randomized controlled trial (topic)'/de OR random*:ab,ti OR nct* OR [randomized controlled trial]/lim)

12 \#9 AND ('cohort analysis' OR 'comparative study'/exp OR 'controlled study'/exp OR 'evaluation study'/de OR 'longitudinal study'/de OR 'major clinical study'/de OR 'prospective study'/de OR 'treatment outcome'/de OR 'between groups':ti,ab OR 'case control*':ti,ab OR cohort*:ti,ab OR compar*:ti,ab OR 'control group*':ti,ab OR 'controlled study':ti,ab OR 'controlled trial':ti,ab OR 'double blind':ti,ab OR 'double blinded':ti,ab OR longitudinal:ti,ab OR 'matched controls':ti,ab OR nonrandomiz*:ti,ab OR prospective:ti,ab OR random*:ti,ab OR versus:ti OR vs:ti)

13 \#10 OR \#11 OR \#12

14 \#5 AND ('dietary supplement'/exp OR 'drug therapy'/exp OR 'enteric feeding'/exp OR 'parenteral nutrition'/exp OR "drug therap*" OR "nutrition team*” OR ((enteric OR enteral OR parenteral) NEAR/2 (feed* OR nutrition* OR nutrient* OR therap*)) OR "Oral nutrition supplement*" OR pharmacotherap* OR ((diet* OR dietitian* OR nutrition*) NEAR/3 (counsel* OR therap*)))

15 \#14 AND ([cochrane review]/lim OR [systematic review]/lim OR [meta analysis]/lim OR cochrane OR 'meta analysis' OR 'meta analyses' OR metaanlysis OR metaanalyses OR search* OR systematic:ti)

16 \#14 AND ('randomized controlled trial'/de OR 'randomized controlled trial (topic)'/de OR random*:ab,ti OR nct* OR [randomized controlled trial]/lim)

17 \#15 OR \#16

18 \#6 OR \#13 OR \#17

19 \#18 NOT (adolescen*:ti OR babies:ti OR baby:ti OR child*:ti OR fetal:ti OR foetal:ti OR infan*:ti OR neonat*:ti OR newborn*:ti OR nicu:ti OR nurser*:ti OR paediatric*:ti OR pediatric*:ti OR pubesc* OR pubert*:ti OR 'school age*':ti OR teen*:ti OR toddler*:ti OR young*:ti OR youth*:ti)

20 \#19 NOT (abstract:nc OR annual:nc OR 'book'/exp OR 'case study'/exp OR conference:nc OR 'conference abstract':it OR 'conference paper'/exp OR 'conference paper':it OR 'conference proceeding':pt OR 'conference review':it OR congress:nc OR 'editorial'/exp OR editorial:it OR 'erratum'/exp OR letter:it OR 'note'/exp OR note:it OR meeting:nc OR sessions:nc OR 'short survey'/exp OR symposium:nc OR [conference abstract]/lim OR [conference paper]/lim OR [conference review]/lim OR [editorial]/lim OR [letter]/lim OR [note]/lim OR [short survey]/lim OR comment:ti OR book:pt OR 'case report'/de OR 'case report':ti OR 'a case':ti OR 'a patient':ti OR 'year old':ti,ab) 


\section{PubMed in process}

1 cachexi*[TI] OR malnourish*[TI] OR malnutrition[TI] OR underfed[TI] OR undernourish*[TI] OR undernutrition[TI]

2 "malnutrition risk" OR "nutrition risk" OR "nutritional risk" OR "risk of malnutrition"

3 "nutrition status" OR "nutritional status" OR "nutrition store*"

4 hospitalis* OR hospitaliz* OR icu OR inpatient* OR "intensive care unit" OR "hospital patient*"

5 (\#1 OR \#2 OR \#3) AND \#4

6 \#5 AND (cochrane OR "meta analysis" OR "meta analyses" OR metaanlysis OR metaanalyses OR search* OR systematic*) AND (inprocess[sb] OR publisher[sb] OR pubmednotmedline[sb]) 7 screen* OR "Malnutrition Screening Tool" OR MST OR "Malnutrition Universal Screening Tool" OR MUST OR "Nutritional Risk Index" OR NRI OR "Nutrition Risk in Critically Ill" OR NUTRIC OR "nutrition risk screening 2002" OR "nrs2002" OR "nrs-2002" OR "short nutritional assessment questionnaire" OR snaq 8 "diagnostic assessment" OR "subjective global assessment" OR sga OR "patient generated subjective global assessment" OR "ps-sga" OR "mini nutritional assessment" OR mna OR "andaspen mce" OR "global leadership initiative on malnutrition" OR glim OR "malnutrition consensus criteria" OR (("academy of nutrition and dietetics" OR "and") AND ("american society for parenteral and enteral nutrition" OR aspen OR "a.s.p.e.n.") AND (consensus OR $\mathrm{mcc})$ ) OR "nutritional assessment" OR "nutrition assessment"

9 \#5 AND (\#7 OR \#8)

10 \#9 AND (cochrane OR "meta analysis" OR "meta analyses" OR metaanlysis OR metaanalyses OR search* OR systematic*) AND (inprocess[sb] OR publisher[sb] OR pubmednotmedline[sb])

11 \#9 AND (random* OR nct*) AND (inprocess[sb] OR publisher[sb] OR pubmednotmedline[sb])

12 \#9 AND ("cohort analysis" OR "between groups" OR "case control*" OR cohort* OR compar* OR "control group*" OR "controlled study" OR "controlled trial" OR "double blind" OR "double blinded" OR longitudinal OR "matched controls" OR nonrandomiz* OR prospective OR random* OR versus[TI] OR vs[TI]) AND (inprocess[SB] OR publisher[SB] OR pubmednotmedline[SB])

13 \#10 OR \#11 OR \#12

14 \#5 AND ("drug therap*" OR "nutrition team*" OR "Oral nutrition supplement*" OR pharmacotherap* OR "enteric feed*" OR "enteric nutrition*" OR "enteric nutrient*" OR "enteric therap*" OR "enteral feed*" OR "enteral nutrition*" OR enteral nutrient"” OR "enteral therap"” OR "parenteral feed*" OR "parenteral nutrition*" OR "parenteral nutrient*" OR "parenteral therap*" OR "diet therap* OR "diet counsel*" OR "nutrition counsel* OR nutrition therap*")

15 \#14 AND (random* OR nct*) AND (inprocess[SB] OR publisher[SB] OR pubmednotmedline[SB])

16 \#14 AND (cochrane OR "meta analysis" OR "meta analyses" OR metaanlysis OR metaanalyses OR search* OR systematic*) AND (inprocess[SB] OR publisher[SB] OR pubmednotmedline[SB])

17 \#15 OR \#16

18 \#6 OR \#13 OR \#17 


\section{Appendix B. Methods}

\section{Inclusion and Exclusion Criteria}

As suggested in the Agency for Healthcare Research and Quality (AHRQ) EPC Methods Guide for Comparative Effectiveness Reviews, the inclusion criteria are listed below in separate categories pertaining to publication type, study design, patient characteristics, test characteristics, and reported data. ${ }^{1}$

\section{Publication Criteria}

1. Full-length articles. The article must be published as a full-length, peer-reviewed study. Abstracts and meeting presentations will not be included because they do not include sufficient details about experimental methods to permit an evaluation of study design and conduct; they may also contain only a subset of measured outcomes. ${ }^{2,3}$ Additionally, it is not uncommon for abstracts that are published as part of conference proceedings to have inconsistencies when compared with the final study publication or to describe studies that are never published as full articles. ${ }^{4-7}$

2. Publication date. Include: For studies of interventions, particularly studies that report on quality metrics such as readmission the search date range will include studies published from 2010 to present. For studies assessing the diagnostic properties of assessment/screening tools the search date range will include studies published from 2000 to present.

3. Redundancy. To avoid double-counting patients, when several reports of the same or overlapping groups of patients are available, only outcome data from the report with the largest number of patients will be included. We will make an exception and include data from a smaller study when it reports data on an outcome that was not provided by the largest report or reports longer followup data for an outcome.

4. English language. When a study with an English abstract is published in a foreign language, the abstract will be assessed against the full set of inclusion/exclusion criteria. If the study appears to fit the inclusion criteria, then we will evaluate whether excluding the study may result in language bias (e.g., if the findings differ from other included studies.) If language bias seems unlikely, the study will be excluded. If a study is selected for inclusion, it will be translated and the data extracted into the evidence tables.

\section{Study Design Criteria}

1. For KQ 1, included studies must be systematic reviews of relevant study designs (RCTs, prospective cohort trials, or cross-sectional studies) that use a valid assessment tool to identify patients at risk of malnutrition.

2. For KQ 2, we will include randomized or non-randomized comparative trials, including prospective cohort trials that report on intermediate and clinical outcomes. Retrospective studies will not be considered for inclusion for this Key Question as these types of study designs are subject to biases that reduce the reliability of the findings.

3. For KQ 3, we will include randomized trials. 
4. For all Key Questions, systematic reviews may also be used as a primary source of data if 3 conditions are met: (1) the review is determined to be at low risk of bias (using Cochrane's ROBIS tool); (2) the included studies would individually meet our inclusion criteria; and (3) our searches did not identify additional, relevant, primary studies that meet our criteria and were not included in the existing review.

\section{Patient Criteria}

1. The patient population for Key Question 1 and 2 will include hospitalized adult patients aged 18 years. Key Question 1 will also consider the following subgroups of patients: patients at risk of malnutrition, patients with baseline malnutrition, and patients with no risk of malnutrition. The population for Key Question 3 will only include patients with a diagnosis of protein-energy malnutrition. Studies enrolling individuals with planned admissions (e.g. undergoing nonemergent elective procedures), patients receiving or who have received hospice services, or pregnant women will not be considered for inclusion in this report.

\section{Intervention Criteria}

1. For Key Question 2 and 3, studies must report on assessment/screening tools (utilized within the U.S., Australia, New Zealand, Canada, and Europe) initiated within the hospital and designed to measure nutritional status.

2. Studies addressing Key Question 2 or 3 must report on an intervention initiated within the hospital and intended to impact nutritional status (See list of example interventions in Table 1). Studies of interventions that are initiated, managed, or implemented by entities either completely or partially external to the hospital setting; or surgical interventions will be excluded. Examples of excluded interventions include but are not limited to ambulatory clinic followup visits, community-based support resources, regulatory policies, and third-party reimbursement programs.

\section{Setting Criteria}

1. Only acute care hospitalization settings. Studies of patients in specialty hospitals (e.g., psychiatric, ophthalmologic, orthopedic, cancer, rehabilitation, long-term acute care) will be excluded.

\section{Data Criteria}

1. The study must report data pertaining to one of the clinical outcomes of interest (mortality, length of stay, functional status, activities of daily living, quality of life, readmission, hospital acquired conditions, and discharge disposition). For KQ 2, diagnostic accuracy factors (sensitivity and specificity), treatment and change in nutrition stores will be considered as intermediate outcomes. Studies reporting only on intermediate outcomes of interest and outcomes exclusive to screening or diagnostic performance will be excluded. 


\section{Grading the Strength of Evidence}

Regardless of whether evidence was synthesized quantitatively or qualitatively, the strength of evidence for each Key Question/body of evidence was assessed for each clinical outcome by using the approach described in the AHRQ Methods Guide. The strength of evidence grades were based on the following factors described in Table B-1.

Table B-1. Factors used to assess strength of evidence

\begin{tabular}{|c|c|}
\hline $\begin{array}{l}\text { Evidence } \\
\text { Category }\end{array}$ & Definition \\
\hline $\begin{array}{l}\text { Study } \\
\text { Limitations }\end{array}$ & $\begin{array}{l}\text { Study limitations takes into account the overall risk of bias rating of all the studies included in the } \\
\text { evidence base, and is rated as low, moderate, or high level of study limitations. }\end{array}$ \\
\hline Consistency & $\begin{array}{l}\text { Consistency of evidence refers to the degree of similarity in the direction of effects or the degree of } \\
\text { similarity in the effect sizes (magnitude of effect) across individual studies within an evidence base, } \\
\text { and is rated as consistent, inconsistent, or unknow/ not applicable in the case of an evidence base } \\
\text { of a single study. }\end{array}$ \\
\hline Directness & $\begin{array}{l}\text { Direct evidence directly compares interventions of interest in populations of interest and measures } \\
\text { patient-oriented outcomes. Evidence can be indirect if the tested intervention differs from the } \\
\text { intervention of interest, the study population differs from the population of interest, the outcomes } \\
\text { differ from those of primary interest, or treatment comparisons have not been tested in head-to-head } \\
\text { comparisons. Directness is rated as direct or indirect. }\end{array}$ \\
\hline Precision & $\begin{array}{l}\text { Precision is the degree of certainty surrounding an estimate of effect with respect to an outcome, } \\
\text { and is primarily assessed by examining the } 95 \% \text { confidence intervals around the summary effect } \\
\text { size. } \\
\text { Cls within the following ranges indicate non-statistical significance, but are considered precise and } \\
\text { should not be downgraded for precision. Further, if a KQ is focused on comparative effectiveness of } \\
\text { two interventions estimates within these bounds support findings of equivalence or no difference. } \\
\text { - Summary estimates using ratio statistics: Lower } \mathrm{Cl} 0.80 \text { to Upper } \mathrm{Cl} 1.25 \text {. } \\
\text { - Summary estimates using standardized mean difference: Lower } \mathrm{Cl}-0.2 \text { to Upper } \mathrm{Cl} 0.2 \text {. } \\
\text { - Summary estimates using raw mean difference: Depends on measure or instrument; default is } \\
\text { Estimates outside of these bounds would be considered imprecise and downgraded for imprecision. } \\
\text { Precision may also take into account the total number of patients in a systematic review. If the total } \\
\text { number of patients included in a systematic review is less than the number of patients generated by } \\
\text { a conventional sample size calculation for a single adequately powered trial, reviewers may } \\
\text { downgrade for precision. This threshold as the "optimal information size" (OIS). Using GRADE } \\
\text { guidance, we considered a sample size of } 1,000 \text { or more as sufficiently high for risk estimates and } \\
400 \text { or more as sufficiently high for continuous estimates. } \\
\text { - If the optimal information size criterion is not met, rate down for imprecision, unless the sample } \\
\text { size is sufficiently large. } \\
\text { - If the OIS criterion is met and the } 95 \% \mathrm{Cl} \text { excludes no effect (i.e. Cl around RR excludes } 1.0 \text { ), } \\
\text { do not rate down for imprecision. } \\
\text { for imprecision if the Cl fails to exclude important benefit or important harm. }\end{array}$ \\
\hline
\end{tabular}

We did not assess the potential for publication bias as the number of studies was not sufficient $(\geq 10)$ for most outcomes in Key Question 3 to perform tests, such as Trim and Fill or Funnel Plot. However, we did assess the potential for selective outcome reporting bias by 
checking the outcomes reported in the included studies for Key Question 3 against the protocols for the studies provided through Clinicaltrials.gov.

Each body of evidence was assigned an overall strength of evidence grade of high, moderate, low, or insufficient based on the definitions presented in Table B-2.

\section{Table B-2. Definitions of the grades of overall strength of evidence}

\begin{tabular}{|l|l|}
\hline Grade & Definition \\
\hline High & $\begin{array}{l}\text { We are very confident that the estimate of effect lies close to the true effect for this outcome. The } \\
\text { body of evidence has few or no deficiencies. We believe that the findings are stable (i.e., another } \\
\text { study would not change the conclusions). }\end{array}$ \\
\hline Loderate & $\begin{array}{l}\text { We are moderately confident that the estimate of effect lies close to the true effect for this outcome. } \\
\text { The body of evidence has some deficiencies. We believe that the findings are likely to be stable, but } \\
\text { some doubt remains. }\end{array}$ \\
\hline $\begin{array}{l}\text { We have limited confidence that the estimate of effect lies close to the true effect for this outcome. } \\
\text { The body of evidence has major or numerous deficiencies (or both). We believe that additional } \\
\text { evidence is needed before concluding either that the findings are stable or that the estimate of effect } \\
\text { is close to the true effect. }\end{array}$ \\
\hline Insufficient & $\begin{array}{l}\text { We have no evidence, we are unable to estimate an effect, or we have no confidence in the estimate } \\
\text { of effect for this outcome. No evidence is available, or the body of evidence has unacceptable } \\
\text { deficiencies, precluding reaching a conclusion. }\end{array}$ \\
\hline
\end{tabular}

\section{Peer Review and Public Commentary}

Peer reviewers with expertise in nutrition were invited to provide written comments on the draft report. The AHRQ Task Order Officer and an Evidence-based Practice Center Associate Editor will also provide comments and editorial review. The draft report will be posted on the AHRQ web site for 4 weeks for public comment. A disposition of comments report with authors' responses to all peer and public review comments will be posted after publication of the final CER on the AHRQ public Web site. 


\section{Appendix C. Excluded Studies}

Table C-1. Studies excluded at full text

\begin{tabular}{|c|c|}
\hline Author & Reason for Exclusion \\
\hline Burgel et al. $2020^{8}$ & Individual study addressing KQ1 \\
\hline Cattani et al. $2020^{9}$ & Not a setting of interest \\
\hline Habaybeh et al. $2020^{10}$ & Not a setting of interest \\
\hline Hsueh et al. $2020^{11}$ & Not a study design of interest \\
\hline Jantharapattana et al. $2020^{12}$ & Not a setting of interest \\
\hline Lima et al. $2020^{13}$ & Individual study addressing KQ1 \\
\hline Matheson et al. $2020^{14}$ & Not a study design of interest \\
\hline Torbahn et al. $2020^{15}$ & Population not of interest \\
\hline Valladares et al. $2020^{16}$ & Not an intervention of interest \\
\hline Xie et al. $2020^{17}$ & Not a setting of interest \\
\hline Becker et al. $2019^{18}$ & Not a study design of interest \\
\hline Gomes et al. $2019^{19}$ & Other exclusion criteria \\
\hline Lovesley et al. $2019^{20}$ & Not a study design of interest \\
\hline Padilla et al. $2019^{21}$ & Population not of interest \\
\hline Rinninella et al. $2019^{22}$ & Population not of interest \\
\hline Thomas et al. $2019^{23}$ & Not a study design of interest \\
\hline Yang et al. $2019^{24}$ & Not a setting of interest \\
\hline Yu et al. $2019^{25}$ & Population not of interest \\
\hline Malafarina et al. $2018^{26}$ & Not a study design of interest \\
\hline Ortíz-Reyes et al. $2018^{27}$ & Not a setting of interest \\
\hline Perman et al. $2018^{28}$ & Population not of interest \\
\hline Rasmussen et al. $2018^{29}$ & Not an intervention of interest \\
\hline Re et al. $2018^{30}$ & Not published in English \\
\hline Shi et al. $2018^{31}$ & Population not of interest \\
\hline Wyers et al. $2018^{32}$ & Population not of interest \\
\hline Cano-Torres et al. $2017^{33}$ & Not a setting of interest \\
\hline Feinberg et al. $2017^{34}$ & Other exclusion criteria \\
\hline García-Rodríguez et al. $2017^{35}$ & Population not of interest \\
\hline Sriram et al. $2017^{36}$ & Not an intervention of interest \\
\hline Aust et al. $2016^{37}$ & Not published in English \\
\hline Baldwin et al. $2016^{38}$ & Population not of interest \\
\hline Bally et al. $2016^{39}$ & Other exclusion criteria \\
\hline Özbilginet al. $2016^{40}$ & Individual study addressing KQ1 \\
\hline Calleja Fernandez et al. $2015^{41}$ & Individual study addressing KQ1 \\
\hline Casals et al. $2015^{42}$ & Not published in English \\
\hline Da Silva Fink et al. $2015^{43}$ & Not a setting of interest \\
\hline Rossi et al. $2015^{44}$ & Not a study design of interest \\
\hline Sun et al. $2015^{45}$ & Not a setting of interest \\
\hline Tapia et al. $2015^{46}$ & Not a study design of interest \\
\hline Agarwal et al. $2013^{47}$ & Individual study addressing KQ1 \\
\hline Lomivorotov et al. $2013^{48}$ & Included in SR for KQ1 \\
\hline Milte et al. $2013^{49}$ & Not an outcome of interest \\
\hline Olveira et al. $2013^{50}$ & Not a study design of interest \\
\hline Omidvari et al. $2013^{51}$ & Not a study design of interest \\
\hline Collins et al. $2012^{52}$ & Population not of interest \\
\hline
\end{tabular}




\begin{tabular}{|c|c|}
\hline Author & Reason for Exclusion \\
\hline Moon et al. $2012^{53}$ & Not an intervention of interest \\
\hline Neelemaat et al. $2012^{54}$ & Not a study design of interest \\
\hline Klek et al. $2011^{55}$ & Population not of interest \\
\hline Klek et al. $2011^{56}$ & Population not of interest \\
\hline Velasco et al. $2011^{57}$ & Included in SR for KQ1 \\
\hline Neelemaat et al. $2010^{58}$ & Not a study design of interest \\
\hline Cansado et al. $2009^{59}$ & Not an outcome of interest \\
\hline Milne et al. $2009^{60}$ & Population not of interest \\
\hline Baldwin et al. $2008^{61}$ & Population not of interest \\
\hline Pronio et al. $2008^{62}$ & Not published in English \\
\hline Koretz et al. $2007^{63}$ & Population not of interest \\
\hline Vanderkroft et al. $2007^{64}$ & Population not of interest \\
\hline Alho Letra Martins et al. $2006^{65}$ & Not a study design of interest \\
\hline De Luis et al. $2006^{66}$ & Not a comparator of interest \\
\hline Miller et al. $2006^{67}$ & Population not of interest \\
\hline Kruizenga et al. $2005^{68}$ & Not a study design of interest \\
\hline Braunschweig et al. $2004^{69}$ & Not a study design of interest \\
\hline Hickson et al. $2004^{70}$ & Population not of interest \\
\hline Rypkema et al. $2004^{71}$ & Population not of interest \\
\hline Kondrup et al. $2003^{72}$ & Not a setting of interest \\
\hline Potter et al. $2001^{73}$ & Not an intervention of interest \\
\hline
\end{tabular}




\section{Appendix D. Characteristics of Included Studies}

Table D-1. Characteristics of systematic reviews assessing association of malnutrition with outcomes

\begin{tabular}{|c|c|c|c|c|c|c|c|c|}
\hline $\begin{array}{l}\text { Author/Year } \\
\text { Objective }\end{array}$ & $\begin{array}{l}\text { Databases } \\
\text { Searched and } \\
\text { Timeframe }\end{array}$ & Study Selection Criteria & $\begin{array}{l}\text { Evidence Base } \\
\text { (n of Included } \\
\text { Studies) } \\
\text { Design of } \\
\text { Included } \\
\text { Studies }\end{array}$ & $\begin{array}{l}\text { ROB of SR } \\
\text { (ROBIS } \\
\text { Rating) }\end{array}$ & $\begin{array}{l}\text { Instrument Used } \\
\text { for ROB of } \\
\text { Included Studies } \\
\text { Overall ROB } \\
\text { Rating }\end{array}$ & $\begin{array}{l}\text { Meta- } \\
\text { Analysis }\end{array}$ & $\begin{array}{l}\text { Qualitative } \\
\text { or } \\
\text { Narrative } \\
\text { Synthesis }\end{array}$ & $\begin{array}{l}\text { GRADE } \\
\text { or Similar } \\
\text { Analysis }\end{array}$ \\
\hline $\begin{array}{l}\text { Dijkink } \\
\text { et al. } 2020^{74} \\
\text { To review the } \\
\text { current } \\
\text { knowledge about } \\
\text { the } \\
\text { pathophysiology, } \\
\text { prevalence, and } \\
\text { effects of } \\
\text { malnutrition in } \\
\text { severely injured } \\
\text { patients. }\end{array}$ & $\begin{array}{l}\text { PubMed and } \\
\text { Embase from } \\
\text { inception to May } \\
2019 \text {. }\end{array}$ & $\begin{array}{l}\text { Inclusion: Full-text studies } \\
\text { published in Dutch, } \\
\text { English, French, and } \\
\text { German without restriction } \\
\text { on publication year. } \\
\text { Included studies either: } \\
\text { (1) described the metabolic } \\
\text { response of malnutrition in } \\
\text { severely injured trauma } \\
\text { patients, or (2) were } \\
\text { clinical cohort studies } \\
\text { describing the prevalence } \\
\text { of malnutrition and its } \\
\text { association with clinical } \\
\text { outcomes in severely } \\
\text { injured trauma patients } \\
\text { during hospital admission. } \\
\text { Excluded: Expert } \\
\text { opinions, conference } \\
\text { papers and letters to the } \\
\text { editor. }\end{array}$ & $\begin{array}{l}13 \text { cohort trials } \\
\text { (2 retrospective, } \\
11 \text { prospective); } \\
10 \text { reported on } \\
\text { outcomes related } \\
\text { to malnutrition }\end{array}$ & Low & $\begin{array}{l}\text { Used MINORS } \\
\text { (Methodological } \\
\text { Index for Non- } \\
\text { randomized } \\
\text { Studies) criteria } \\
\text { "Generally" low } \\
\text { ROB; however, } \\
\text { rating tool does not } \\
\text { assess if study } \\
\text { controlled for } \\
\text { confounders. }\end{array}$ & No & Yes & No \\
\hline
\end{tabular}




\begin{tabular}{|c|c|c|c|c|c|c|c|c|}
\hline $\begin{array}{l}\text { Author/Year } \\
\text { Objective }\end{array}$ & $\begin{array}{l}\text { Databases } \\
\text { Searched and } \\
\text { Timeframe }\end{array}$ & Study Selection Criteria & $\begin{array}{l}\text { Evidence Base } \\
\text { ( } \mathrm{n} \text { of Included } \\
\text { Studies) } \\
\text { Design of } \\
\text { Included } \\
\text { Studies } \\
\end{array}$ & $\begin{array}{l}\text { ROB of SR } \\
\text { (ROBIS } \\
\text { Rating) }\end{array}$ & $\begin{array}{l}\text { Instrument Used } \\
\text { for ROB of } \\
\text { Included Studies } \\
\text { Overall ROB } \\
\text { Rating }\end{array}$ & $\begin{array}{l}\text { Meta- } \\
\text { Analysis }\end{array}$ & $\begin{array}{l}\text { Qualitative } \\
\text { or } \\
\text { Narrative } \\
\text { Synthesis }\end{array}$ & $\begin{array}{l}\text { GRADE } \\
\text { or Similar } \\
\text { Analysis }\end{array}$ \\
\hline $\begin{array}{l}\text { Ney et al., } \\
2019^{75} \\
\text { A systematic } \\
\text { review with the } \\
\text { aim of } \\
\text { summarizing: the } \\
\text { varying } \\
\text { definitions of } \\
\text { malnutrition } \\
\text { across studies, } \\
\text { the available } \\
\text { evidence for } \\
\text { nutritional } \\
\text { screening tools } \\
\text { (NST) and the } \\
\text { ability of NSTs } \\
\text { and nutritional } \\
\text { assessment } \\
\text { tools (NATs) to } \\
\text { predict clinical } \\
\text { outcomes in } \\
\text { patients with } \\
\text { cirrhosis. }\end{array}$ & $\begin{array}{l}\text { PubMed (1966- } \\
\text { 2018), EMBASE } \\
\text { (1974-2018) and } \\
\text { Web of Science } \\
\text { (1990-2018). } \\
\text { Bibliographies of } \\
\text { the included } \\
\text { studies and } \\
\text { relevant review } \\
\text { papers were } \\
\text { searched by hand } \\
\text { for any further } \\
\text { studies. The final } \\
\text { search was } \\
\text { completed on } \\
\text { February 14, } \\
2018 .\end{array}$ & $\begin{array}{l}\text { Inclusion: (a) full-text } \\
\text { English language articles, } \\
\text { (b) patients with cirrhosis } \\
\geq 16 \text { years of age, } \\
\text { (c) studies assessing } \\
\text { clinical outcomes as } \\
\text { predicted by NSTs or } \\
\text { NATs and/or (d) studies } \\
\text { measuring the validity of } \\
\text { NSTs for diagnosing } \\
\text { malnutrition. } \\
\text { Exclusion: (a) } 25 \% \text { of } \\
\text { patients with hepatocellular } \\
\text { carcinoma, (b) studies } \\
\text { investigating NATs that } \\
\text { were primarily comprised } \\
\text { of laboratory-based } \\
\text { parameters (ie controlling } \\
\text { nutritional status score), } \\
\text { (c) studies without formal } \\
\text { nutritional assessment/ } \\
\text { screening objectives and } \\
\text { (d) body mass index (BMI) } \\
\text { as the sole marker of } \\
\text { nutrition status. }\end{array}$ & $\begin{array}{l}47 \text { cross- } \\
\text { sectional; } \\
8 \text { reported } \\
\text { outcomes for } \\
\text { tools of interest } \\
\text { (SGA) }\end{array}$ & Low & $\begin{array}{l}\text { Ottawa Newcastle } \\
\text { Assessment Scale } \\
\text { (ONS) } \\
\text { Moderate ROB; loss } \\
\text { to follow-up }\end{array}$ & $\begin{array}{l}\text { Yes, } \\
\text { mortality }\end{array}$ & Yes & No \\
\hline
\end{tabular}




\begin{tabular}{|c|c|c|c|c|c|c|c|c|}
\hline $\begin{array}{l}\text { Author/Year } \\
\text { Objective }\end{array}$ & $\begin{array}{l}\text { Databases } \\
\text { Searched and } \\
\text { Timeframe }\end{array}$ & Study Selection Criteria & $\begin{array}{l}\text { Evidence Base } \\
\text { (n of Included } \\
\text { Studies) } \\
\text { Design of } \\
\text { Included } \\
\text { Studies }\end{array}$ & $\begin{array}{l}\text { ROB of SR } \\
\text { (ROBIS } \\
\text { Rating) }\end{array}$ & $\begin{array}{l}\text { Instrument Used } \\
\text { for ROB of } \\
\text { Included Studies } \\
\text { Overall ROB } \\
\text { Rating }\end{array}$ & $\begin{array}{l}\text { Meta- } \\
\text { Analysis }\end{array}$ & $\begin{array}{l}\text { Qualitative } \\
\text { or } \\
\text { Narrative } \\
\text { Synthesis }\end{array}$ & $\begin{array}{l}\text { GRADE } \\
\text { or Similar } \\
\text { Analysis }\end{array}$ \\
\hline $\begin{array}{l}\text { Muscaritoli et al. } \\
2017^{76}\end{array}$ & $\begin{array}{l}\text { Cochrane Central } \\
\text { Register of } \\
\text { Controlled Trials } \\
\text { (CENTRAL), The } \\
\text { Cochrane } \\
\text { Database of } \\
\text { Systematic } \\
\text { Reviews (CDSR), } \\
\text { Database of } \\
\text { Abstracts of } \\
\text { Reviews of } \\
\text { Effects (DARE), } \\
\text { MEDLINE (1946 } \\
\text { onwards), and } \\
\text { ClinicalTrials.gov. }\end{array}$ & $\begin{array}{l}\text { Prospective cohort studies } \\
\text { of adult hospital patients } \\
\text { with any disease, during } \\
\text { their hospital stay or during } \\
\text { follow-up after discharge. }\end{array}$ & $\begin{array}{l}15 \text { studies, } \\
12 \text { prospective } \\
\text { cohort studies } \\
\text { and } 3 \text { database } \\
\text { studies }\end{array}$ & Low & $\begin{array}{l}\text { Modified Cochrane } \\
\text { Risk of Bias tool, } \\
\text { using three out of } \\
\text { six original domains } \\
\text { (blinding, selective } \\
\text { data reporting, } \\
\text { selective outcome } \\
\text { reporting) and two } \\
\text { additional domains } \\
\text { (comparability of } \\
\text { groups and } \\
\text { confounding } \\
\text { factors) were used } \\
\text { to assess the risk of } \\
\text { bias in the included } \\
\text { non-randomized } \\
\text { studies. } \\
\text { All studies rated as } \\
\text { High ROB due to } \\
\text { lack of } \\
\text { comparability of } \\
\text { included patients } \\
\text { and lack of or not } \\
\text { clearly reporting if } \\
\text { confounders were } \\
\text { controlled. }\end{array}$ & No & Yes & No \\
\hline
\end{tabular}




\begin{tabular}{|c|c|c|c|c|c|c|c|c|}
\hline $\begin{array}{l}\text { Author/Year } \\
\text { Objective }\end{array}$ & $\begin{array}{l}\text { Databases } \\
\text { Searched and } \\
\text { Timeframe }\end{array}$ & Study Selection Criteria & $\begin{array}{l}\text { Evidence Base } \\
\text { (n of Included } \\
\text { Studies) } \\
\text { Design of } \\
\text { Included } \\
\text { Studies }\end{array}$ & $\begin{array}{l}\text { ROB of SR } \\
\text { (ROBIS } \\
\text { Rating) }\end{array}$ & $\begin{array}{l}\text { Instrument Used } \\
\text { for ROB of } \\
\text { Included Studies } \\
\text { Overall ROB } \\
\text { Rating }\end{array}$ & \begin{tabular}{|l|} 
Meta- \\
Analysis
\end{tabular} & $\begin{array}{l}\text { Qualitative } \\
\text { or } \\
\text { Narrative } \\
\text { Synthesis }\end{array}$ & $\begin{array}{l}\text { GRADE } \\
\text { or Similar } \\
\text { Analysis }\end{array}$ \\
\hline $\begin{array}{l}\text { Lew et al. } 2016^{77} \\
\text { To assess the } \\
\text { association } \\
\text { between } \\
\text { malnutrition and } \\
\text { clinical outcomes } \\
\text { in the ICU. }\end{array}$ & $\begin{array}{l}\text { PubMed, } \\
\text { CINAHL, Scopus, } \\
\text { and Cochrane } \\
\text { Library on } \\
\text { August 1, 2014; } \\
\text { additionally } \\
\text { searched } \\
\text { reference lists of } \\
\text { the articles that } \\
\text { were included in } \\
\text { this systematic } \\
\text { review were hand } \\
\text { searched along } \\
\text { with the table of } \\
\text { contents of } \\
\text { Critical Care } \\
\text { Medicine, Journal } \\
\text { of Parenteral and } \\
\text { Enteral Nutrition, } \\
\text { and Nutrition in } \\
\text { Clinical Practice } \\
\text { from inception to } \\
\text { August 2014. }\end{array}$ & $\begin{array}{l}\text { Inclusion: Patients } \\
>18 \text { years and not } \\
\text { pregnant admitted to the } \\
\text { ICU who were screened/ } \\
\text { assessed for malnutrition } \\
\text { using a nutrition screening } \\
\text { or assessment tool and } \\
\text { whose outcomes were } \\
\text { based on the results of } \\
\text { nutrition screening or } \\
\text { assessment (i.e., not at } \\
\text { risk of malnutrition vs at } \\
\text { risk of malnutrition or well- } \\
\text { nourished vs } \\
\text { malnourished). } \\
\text { Exclusion: Articles that } \\
\text { measured the prognostic } \\
\text { value of individual } \\
\text { biochemical markers or } \\
\text { anthropometric } \\
\text { measurements, articles } \\
\text { that did not report on the } \\
\text { prevalence of malnutrition, } \\
\text { relevant clinical outcomes, } \\
\text { and/or results specific to } \\
\text { patients in the ICU. Articles } \\
\text { that did not compare the } \\
\text { clinical outcomes between } \\
\text { at-risk patients and not-at- } \\
\text { risk patients for } \\
\text { malnutrition and between } \\
\text { well-nourished and } \\
\text { malnourished patients. }\end{array}$ & $\begin{array}{l}20 \text { prospective } \\
\text { cohort trials (of } \\
\text { which } 15 \text { were } \\
\text { used to assess } \\
\text { outcomes) }\end{array}$ & Low & $\begin{array}{l}\text { Instrument } \\
\text { described in } \\
\text { Laupacis et al. } 1994 \\
\text { that considered } \\
\text { representativeness } \\
\text { of patients, } \\
\text { sufficient follow-up, } \\
\text { blinding of outcome } \\
\text { assessors, and } \\
\text { controlling for } \\
\text { confounders. } \\
6 \text { studies rated as } \\
\text { "Low" ROB and } \\
9 \text { rated as } \\
\text { "Possible" ROB. } \\
\text { The authors of the } \\
\text { review only } \\
\text { considered studies } \\
\text { with Low ROB when } \\
\text { drawing } \\
\text { conclusions. }\end{array}$ & No & Yes & No \\
\hline
\end{tabular}




\begin{tabular}{|c|c|c|c|c|c|c|c|c|}
\hline $\begin{array}{l}\text { Author/Year } \\
\text { Objective }\end{array}$ & $\begin{array}{l}\text { Databases } \\
\text { Searched and } \\
\text { Timeframe }\end{array}$ & Study Selection Criteria & $\begin{array}{l}\text { Evidence Base } \\
\text { ( } n \text { of Included } \\
\text { Studies) } \\
\text { Design of } \\
\text { Included } \\
\text { Studies }\end{array}$ & $\begin{array}{l}\text { ROB of SR } \\
\text { (ROBIS } \\
\text { Rating) }\end{array}$ & $\begin{array}{l}\text { Instrument Used } \\
\text { for ROB of } \\
\text { Included Studies } \\
\text { Overall ROB } \\
\text { Rating }\end{array}$ & $\begin{array}{l}\text { Meta- } \\
\text { Analysis }\end{array}$ & $\begin{array}{l}\text { Qualitative } \\
\text { or } \\
\text { Narrative } \\
\text { Synthesis }\end{array}$ & $\begin{array}{l}\text { GRADE } \\
\text { or Similar } \\
\text { Analysis }\end{array}$ \\
\hline Lin et al. $2016^{78}$ & $\begin{array}{l}\text { PubMed, } \\
\text { Embase, Web of } \\
\text { Science and } \\
\text { ScienceDirect } \\
\text { from inception to } \\
\text { May } 2015 .\end{array}$ & $\begin{array}{l}\text { Only studies of adults } \geq 18 \\
\text { years published in English } \\
\text { that (1) assessment of the } \\
\text { nutritional status of } \\
\text { patients with HF using } \\
\text { multidimensional } \\
\text { evaluation tools or } \\
\text { multidimensional } \\
\text { evaluation technologies } \\
\text { recommended by ASPEN; } \\
\text { (2) assessment of } \\
\text { prognostic value } \\
\text { parameters [e.g. length of } \\
\text { stay (LOS), mortality, } \\
\text { hospitalization and } \\
\text { complications; (3) available } \\
\text { full text; and (4) cohort or } \\
\text { cross-sectional studies. }\end{array}$ & $\begin{array}{l}17 \text { trials of which } \\
11 \text { trials were of } \\
\text { hospitalized } \\
\text { patients } \\
\text { Prospective } \\
\text { cohort (12); } \\
\text { retrospective } \\
\text { cohort (5) }\end{array}$ & Low & $\begin{array}{l}\text { Ottawa Newcastle } \\
\text { Assessment Scale } \\
\text { (ONS) } \\
10 \text { Fair ROB; } \\
7 \text { Good ROB }\end{array}$ & $\begin{array}{l}\text { Yes, only } \\
\text { for } \\
\text { mortality }\end{array}$ & Yes & No \\
\hline $\begin{array}{l}\text { Gupta et al. } \\
2011^{79}\end{array}$ & $\begin{array}{l}\text { MEDLINE from } \\
\text { inception to } \\
\text { December } 2010 ; \\
\text { bibliographies of } \\
\text { selected papers. }\end{array}$ & $\begin{array}{l}\text { Only studies published in } \\
\text { English, reported data } \\
\text { collected in humans with } \\
\text { cancer, had nutritional } \\
\text { status/assessment/ } \\
\text { screening as one of the } \\
\text { predictor variables, had } \\
\text { LOS as one of the } \\
\text { outcome measures, and } \\
\text { utilized any of the following } \\
\text { study designs (case- } \\
\text { control, cohort, cross- } \\
\text { sectional, prospective, } \\
\text { retrospective, case series, } \\
\text { longitudinal, clinical trial, } \\
\text { meta-analysis). There } \\
\text { were no restrictions in } \\
\text { terms of age, ethnicity, or } \\
\text { type or stage of cancer. }\end{array}$ & $\begin{array}{l}8 \text { trials of } \\
21 \text { trials reported } \\
\text { in association } \\
\text { between } \\
\text { screening/ } \\
\text { assessment tool } \\
\text { and LOS (other } \\
\text { studies only } \\
\text { considered } \\
\text { biomarkers }\end{array}$ & $\begin{array}{l}\text { High; review } \\
\text { did not } \\
\text { assess ROB } \\
\text { of included } \\
\text { studies } \\
\text { **Authors of } \\
\text { review did } \\
\text { report on key } \\
\text { elements, } \\
\text { such as } \\
\text { study design, } \\
\text { confounder } \\
\text { analysis. }\end{array}$ & $\begin{array}{l}\text { NR; based on } \\
\text { information } \\
\text { provided by } \\
\text { authors, studies } \\
\text { most studies were } \\
\text { high ROB due to } \\
\text { lack of cofounder } \\
\text { analysis. }\end{array}$ & No & Yes & No \\
\hline
\end{tabular}


Table D-2. Characteristics of studies and patients included in systematic reviews on association of malnutrition on outcomes

\begin{tabular}{|c|c|c|c|c|c|}
\hline $\begin{array}{l}\text { Authorl } \\
\text { Year }\end{array}$ & $\begin{array}{l}\text { Number of Patients } \\
\text { Length of Follow-up } \\
\text { Hospital Setting, } \\
\text { n Studies }\end{array}$ & $\begin{array}{l}\text { Patient Age } \\
\text { Gender } \\
\text { Ethnicity } \\
\text { Socioeconomic } \\
\text { Status (SES) }\end{array}$ & $\begin{array}{l}\text { Underlying Condition, } \\
\mathbf{n} \text { Studies } \\
\text { Severity of Disease }\end{array}$ & $\begin{array}{l}\text { Nutritional Screening/ } \\
\text { Assessment Tool(s) }\end{array}$ & $\%$ Malnourished \\
\hline $\begin{array}{l}\text { Dijkink } \\
\text { et al. } 2020^{74}\end{array}$ & $\begin{array}{l}\mathrm{N}=9,167 \\
\mathrm{NR} \\
\mathrm{NR}\end{array}$ & $\begin{array}{l}\text { Mean: } 61.8, \\
\text { range } 27 \text { to } 84 \\
\% \text { Male: } 54.8 \\
\text { NR } \\
\text { NR }\end{array}$ & $\begin{array}{l}\text { General trauma patients with } \\
\text { severe injuries: } 6 \text { studies } \\
\text { Geriatric trauma patients with } \\
\text { severe injuries: } 4 \text { studies } \\
\text { Severe injuries }\end{array}$ & $\begin{array}{l}\text { Visceral proteins: } 4 \text { studies } \\
\text { NRS: } 2 \text { studies } \\
\text { MNA: } 4 \text { studies } \\
\text { SGA: } 2 \text { study } \\
\text { Anthropometric measures: } 1 \text { study }\end{array}$ & $\begin{array}{l}\text { All pts: } 7 \text { to } 76 \% \\
\text { Geriatric pts: } 7 \text { to } 62.5 \% \\
\% \text { Geriatric pts at risk: } 35.6 \\
\text { to } 60 \%\end{array}$ \\
\hline $\begin{array}{l}\text { Ney et al. } \\
2019^{75}\end{array}$ & $\begin{array}{l}\mathrm{N}=611 \text { among studies } \\
\text { of SGA } \\
\text { NR } \\
\text { Inpatient }\end{array}$ & $\begin{array}{l}\text { Mean: } 52 \\
\% \text { Male: } 67.2 \% \\
\text { NR } \\
\text { NR }\end{array}$ & $\begin{array}{l}\text { Cirrhosis } \\
\text { Model for End Stage Liver } \\
\text { Disease (MELD): } 14.8 \\
\text { Child-Pugh (CP): } 26 \% \text { CP A, } \\
41.6 \% \text { was CP B and } 32.5 \% \text { was } \\
\text { CP C }\end{array}$ & SGA: 8 studies & $8.0 \%$ to $100 \%$ \\
\hline $\begin{array}{l}\text { Muscaritoli } \\
\text { et al. } 2017^{76}\end{array}$ & $\begin{array}{l}\mathrm{N}=20,775 \\
\mathrm{NR} \\
\mathrm{NR}\end{array}$ & $\begin{array}{l}\text { NR } \\
\text { NR } \\
\text { NR } \\
\text { NR }\end{array}$ & $\begin{array}{l}\text { Various conditions/general } \\
\text { medicine: } 7 \\
\text { COPD: } 3 \\
\text { Heart failure: } 4 \\
\text { Pneumonia: } 1 \\
\text { NR } \\
\end{array}$ & $\begin{array}{l}\text { SGA: } 3 \text { studies } \\
\text { NRI: } 1 \text { study } \\
\text { MNA: } 2 \text { studies } \\
\text { Weight/weight loss/BMI/ } \\
\text { anthropometric: } 8 \text { studies } \\
\text { MUST: } 1 \text { study }\end{array}$ & NR \\
\hline $\begin{array}{l}\text { Lew et al. } \\
2016^{77}\end{array}$ & $\begin{array}{l}\mathrm{N}=4,228 \\
\text { Follow-up: Entire ICU } \\
\text { admission period to } \\
\text { 1-year post-discharge } \\
\text { Medical ICU: } 10 \text { studies } \\
\text { Surgical ICU: } 1 \text { study } \\
\text { Medical vs. Surgical } \\
\text { Not Reported: } \\
10 \text { studies }\end{array}$ & $\begin{array}{l}\text { Mean: } 59 \text { years } \\
\text { NR } \\
\text { NR } \\
\text { NR }\end{array}$ & $\begin{array}{l}\text { Heterogeneous condition: } \\
12 \text { studies } \\
\text { Elderly: } 3 \text { studies } \\
\text { Post-Liver transplant: } 2 \text { studies } \\
\text { Cardiac patients: } 2 \text { studies } \\
\text { Acute kidney injury: } 1 \text { study } \\
\text { APACHE Score: Range } 12 \text { to } 25\end{array}$ & $\begin{array}{l}\text { SGA: } 10 \text { studies } \\
\text { MNA: } 2 \text { studies } \\
\text { Nutrition Risk Screening-2002: } \\
4 \text { studies } \\
\text { MUST: } 3 \text { studies } \\
\text { MNA-SF: } 1 \text { study } \\
\text { Prognostic Inflammatory and } \\
\text { Nutritional Index (PINI): } 1 \text { study } \\
\text { Short Nutritional Assessment } \\
\text { Questionnaire (SNAQ]): } 1 \text { study }\end{array}$ & $\begin{array}{l}\text { Heterogeneous group } \\
\text { (11 studies): } 37.8 \% \text { to } \\
78.1 \% \\
\text { Elderly ( } 1 \text { study): } 23.2 \% \text { to } \\
34.4 \% \\
\text { Cardiac surgery ( } 1 \text { study): } \\
5.0 \% \text { to } 20.0 \% \\
\text { Liver transplantation } \\
\text { (2 studies): } 52.6 \% \\
\text { Acute kidney injury } \\
\text { (1 study): } 82.0 \%\end{array}$ \\
\hline
\end{tabular}




\begin{tabular}{|c|c|c|c|c|c|}
\hline $\begin{array}{l}\text { Authorl } \\
\text { Year }\end{array}$ & $\begin{array}{l}\text { Number of Patients } \\
\text { Length of Follow-up } \\
\text { Hospital Setting, } \\
\text { n Studies }\end{array}$ & $\begin{array}{l}\text { Patient Age } \\
\text { Gender } \\
\text { Ethnicity } \\
\text { Socioeconomic } \\
\text { Status (SES) }\end{array}$ & $\begin{array}{l}\text { Underlying Condition, } \\
\text { n Studies } \\
\text { Severity of Disease }\end{array}$ & $\begin{array}{l}\text { Nutritional Screening/ } \\
\text { Assessment Tool(s) }\end{array}$ & $\%$ Malnourished \\
\hline $\begin{array}{l}\text { Lin et al. } \\
2016^{78}\end{array}$ & $\begin{array}{l}\mathrm{N}=4,303 \\
\text { Follow-up (median } \\
\text { months): } 27, \text { range } 6 \text { to } \\
68 \\
\mathrm{NR}\end{array}$ & $\begin{array}{l}\text { Mean } 64 \text { years } \\
46 \% \text { male } \\
\text { NR } \\
\text { NR }\end{array}$ & $\begin{array}{l}\text { Heart failure (17) } \\
\text { Advanced HF: } 2 \text { studies } \\
\text { Acute decompensated HF: } \\
3 \text { studies } \\
\text { Stable HF: } 5 \text { studies } \\
\text { All types HF: } 7 \text { studies }\end{array}$ & $\begin{array}{l}\text { MNA: } 5 \text { studies } \\
\text { GNRI: } 4 \text { studies } \\
\text { NRI: } 3 \text { studies } \\
\text { MNA-SF: } 2 \text { studies } \\
\text { SCORE: } 1 \text { study } \\
\text { NRS: } 1 \text { study } \\
\text { CONUT: } 1 \text { study } \\
\text { PNI: } 1 \text { study } \\
\text { Other: } 2 \text { studies }\end{array}$ & $\begin{array}{l}\text { General: } 16 \text { to } 90 \% \\
\text { By tool: } \\
\text { MNA: } 16 \text { to } 90 \% \\
\text { GNRI: } 22 \text { to } 48 \% \\
\text { NRI: } 23 \text { to } 90 \% \\
\text { NRS: } 57.3 \%\end{array}$ \\
\hline $\begin{array}{l}\text { Gupta et al. } \\
2011^{79}\end{array}$ & $\begin{array}{l}\mathrm{N}=2,153 \\
\mathrm{NR} \\
\mathrm{NR}\end{array}$ & $\begin{array}{l}\text { NR } \\
\text { NR } \\
\text { NR } \\
\text { NR }\end{array}$ & $\begin{array}{l}\text { Gastrointestinal cancer (4) } \\
\text { Gynecologic cancer (1) } \\
\text { Head and neck cancer (1) } \\
\text { Multiple myeloma (1) } \\
\text { Lymphoma (1) } \\
\text { NR }\end{array}$ & $\begin{array}{l}\text { SGA: } 6 \text { studies } \\
\text { PG-SGA: } 2 \text { studies }\end{array}$ & $\begin{array}{l}\text { Moderate to severe MN: } \\
898(42 \%)\end{array}$ \\
\hline
\end{tabular}

$\mathrm{CI}=$ confidence interval; $\mathrm{LOS}=$ length of stay; MNA = mini nutritional assessment; MUST $=$ malnutrition universal screening tool; NO indicates no association identified or no important difference found between malnourished and well-nourished; NR = not reported; NRS-2002 = nutrition risk screening 2002; PG-SGA = patient-generated subjective global assessment; PINI = Prognostic Inflammatory and Nutritional Index; RCT = randomized controlled trial; SD = standard deviation; SE = standard error; SES = socioeconomic status; $\mathrm{SGA}=$ subjective global assessment; SNAQ = Short Nutritional Assessment Questionnaire; VP = visceral proteins 
Table D-3. Characteristics of studies and patients of effectiveness of interventions for malnutrition

\begin{tabular}{|c|c|c|c|c|c|c|c|}
\hline $\begin{array}{l}\text { Author/Year } \\
\text { Study Design } \\
\text { Country/ } \\
\text { Setting }\end{array}$ & Objective & $\begin{array}{l}\text { Patient Inclusion/ } \\
\text { Exclusion Criteria }\end{array}$ & $\begin{array}{l}\text { Intervention } \\
\text { Control }\end{array}$ & $\begin{array}{l}\text { Number of } \\
\text { Patients per } \\
\text { Study Arm } \\
\text { Follow-up } \\
\text { Duration }\end{array}$ & $\begin{array}{l}\text { Age } \\
\text { Gender } \\
\text { Race/Ethnicity } \\
\text { Socioeconomic } \\
\text { Status (SES) }\end{array}$ & $\begin{array}{l}\text { Screening } \\
\text { Instrument } \\
\text { Nutritional Status } \\
\text { BMI } \\
\text { Weight } \\
\end{array}$ & $\begin{array}{l}\text { Underlying } \\
\text { Condition } \\
\text { Disease } \\
\text { Severity }\end{array}$ \\
\hline $\begin{array}{l}\text { Bonilla- } \\
\text { Palomas et al. } \\
2016^{80} \\
\text { RCT } \\
\text { Spain }\end{array}$ & $\begin{array}{l}\text { Assess whether a } \\
\text { nutritional } \\
\text { intervention of diet } \\
\text { optimization, } \\
\text { recommendations, } \\
\text { and nutritional } \\
\text { supplement } \\
\text { prescriptions versus } \\
\text { standard care in } \\
\text { malnourished } \\
\text { hospitalized patients } \\
\text { with HF benefits } \\
\text { morbidity and } \\
\text { mortality. }\end{array}$ & $\begin{array}{l}\text { Included patients aged } \\
\text { over } 18 \text { years who are } \\
\text { admitted for acute HF, } \\
\text { whether chronic and } \\
\text { uncompensated or of } \\
\text { new onset, and in a } \\
\text { state of malnutrition } \\
\text { (score on the MNA } \\
<17 \text { points). } \\
\text { Excluded pregnant } \\
\text { women, patients with } \\
\text { chronic renal failure in } \\
\text { dialysis, patients } \\
\text { already receiving } \\
\text { nutritional treatment, } \\
\text { patients with } \\
\text { concomitant disease } \\
\text { who, regardless of HF } \\
\text { itself, have a life } \\
\text { expectancy of less } \\
\text { than } 1 \text { year, patients } \\
\text { participating in other } \\
\text { clinical trials, patients } \\
\text { who undergo surgery } \\
\text { or percutaneous } \\
\text { coronary intervention } \\
\text { during their hospital } \\
\text { stay to correct the } \\
\text { cause of acute HF, } \\
\text { and patients whose } \\
\text { clinical status means } \\
\text { that it is impossible to } \\
\text { perform the nutritional } \\
\text { assessment as } \\
\text { established in the } \\
\text { study protocol or who }\end{array}$ & $\begin{array}{l}\text { Treatment (Tx): } \\
\text { Physician specialist } \\
\text { with assistance by a } \\
\text { nutritionist provided } \\
\text { diet optimization, } \\
\text { specific } \\
\text { recommendations, } \\
\text { and nutritional } \\
\text { supplement } \\
\text { prescriptions in } \\
\text { cases in which } \\
\text { nutritional goals } \\
\text { were not reached for } \\
\text { patients. } \\
\text { Control group (CG): } \\
\text { Conventional } \\
\text { treatment for HF }\end{array}$ & $\begin{array}{l}\text { Tx: } 61 \\
\text { CG: } 59 \\
12 \text { months }\end{array}$ & $\begin{array}{l}\text { Age, mean (SD): } \\
\text { Tx: } 78.6(7.1) ; \\
\text { CG: } 79.8(7.0) \\
p=0.38 \\
\text { Female gender, } \\
\text { n (\%): } \\
\text { Tx: } 38(64.4 \%) ; \\
\text { CG: } 37(60.7 \%) \\
p=0.67 \\
\text { Race/Ethnicity: } \\
\text { NR } \\
\text { SES: NR }\end{array}$ & $\begin{array}{l}\text { MNA score, mean } \\
\text { (SD): } \\
\text { Tx: } 14.4(2.3) \text {; CG: } \\
14.1(2.6), p=0.41 \\
\text { BMI in } \mathrm{kg} / \mathrm{m}^{2} \text {, } \\
\text { mean (SD): } \\
\text { Tx: } 25.5(5.4) \text {; CG: } \\
24.7(5.1), p=0.41\end{array}$ & $\begin{array}{l}\text { Comorbidities, } \\
\text { n (\%): } \\
\text { COPD: Tx: } 11 \\
\text { (18.6\%); CG: } 7 \\
\text { (11.5\%), p=0.27 } \\
\text { Decompensated } \\
\text { CHF: Tx: } 38 \\
\text { (64.4\%); CG: } 40 \\
\text { (65.6\%), p=0.89 } \\
\text { Diabetes: Tx: } 28 \\
\text { (47.5\%); CG: } 30 \\
\text { (49.2\%), p=0.85 } \\
\text { HLD: Tx: } 19 \\
\text { (32.2\%); CG: } 16 \\
\text { (26.2\%), p=0.47 } \\
\text { HTN: Tx: } 41 \\
\text { (69.5\%); CG: } 49 \\
\text { (80.3\%), p=0.17 } \\
\text { Charlson } \\
\text { comorbidity } \\
\text { index, mean } \\
\text { (SD): } \\
\text { Tx: } 3.8 \text { (2.0); } \\
\text { CG: } 4.0(2.2), \\
\text { p=0.63 }\end{array}$ \\
\hline
\end{tabular}




\begin{tabular}{|c|c|c|c|c|c|c|c|}
\hline $\begin{array}{l}\text { Author/Year } \\
\text { Study Design } \\
\text { Country/ } \\
\text { Setting }\end{array}$ & Objective & $\begin{array}{l}\text { Patient Inclusion/ } \\
\text { Exclusion Criteria }\end{array}$ & $\begin{array}{l}\text { Intervention } \\
\text { Control }\end{array}$ & $\begin{array}{l}\text { Number of } \\
\text { Patients per } \\
\text { Study Arm } \\
\text { Follow-up } \\
\text { Duration }\end{array}$ & $\begin{array}{l}\text { Age } \\
\text { Gender } \\
\text { Race/Ethnicity } \\
\text { Socioeconomic } \\
\text { Status (SES) }\end{array}$ & $\begin{array}{l}\text { Screening } \\
\text { Instrument } \\
\text { Nutritional Status } \\
\text { BMI } \\
\text { Weight }\end{array}$ & $\begin{array}{l}\text { Underlying } \\
\text { Condition } \\
\text { Disease } \\
\text { Severity }\end{array}$ \\
\hline & & $\begin{array}{l}\text { do not provide their } \\
\text { consent for such } \\
\text { procedures. }\end{array}$ & & & & & \\
\hline $\begin{array}{l}\text { Deutz et al. } \\
2016^{81} \\
\text { RCT } \\
\text { USA }\end{array}$ & $\begin{array}{l}\text { To evaluate a high- } \\
\text { protein ONS } \\
\text { containing beta- } \\
\text { hydroxybeta- } \\
\text { methylbutyrate on } \\
\text { post discharge } \\
\text { outcomes of } \\
\text { nonelective } \\
\text { readmission and } \\
\text { mortality in } \\
\text { malnourished, } \\
\text { hospitalized older } \\
\text { adults. }\end{array}$ & $\begin{array}{l}\text { Included patients aged } \\
>65 \text { years with a } \\
\text { recent hospital } \\
\text { admission (within } \\
72 \mathrm{~h} \text { ) and a primary } \\
\text { diagnosis of CHF, } \\
\text { AMI, PNA, or COPD. } \\
\text { Patients were required } \\
\text { to have a Subjective } \\
\text { Global Assessment } \\
\text { (SGA) class of B } \\
\text { (moderate or } \\
\text { suspected } \\
\text { malnutrition) or C } \\
\text { (severe malnutrition). } \\
\text { Excluded patients } \\
\text { diabetes mellitus } \\
\text { (type } 1 \text { or } 2 \text { ) due to } \\
\text { product composition } \\
\text { not intended for } \\
\text { patients with diabetes } \\
\text { mellitus; current active } \\
\text { or treated cancer, and } \\
\text { impaired renal or liver } \\
\text { function. }\end{array}$ & $\begin{array}{l}\text { Oral Nutrition } \\
\text { Supplement (ONS): } \\
\text { High-protein ONS } \\
\text { containing beta- } \\
\text { hydroxybeta- } \\
\text { methylbutyrate } \\
\text { Control group (CG): } \\
\text { Placebo }\end{array}$ & $\begin{array}{l}\text { ONS: } 309 \\
\text { CG: } 313 \\
90 \text { days }\end{array}$ & $\begin{array}{l}\text { Age, mean (SD): } \\
\text { ONS: } 78.1(8.6) \text {; } \\
\text { CG: } 77.7(8.2), \\
\text { p=NS } \\
\text { Female gender, } \\
\text { n (\%): } \\
\text { ONS: } 160 \\
\text { (51.8\%); CG: } \\
\text { 164 (52.4\%), } \\
\text { p=NS } \\
\text { Race, n (\%): } \\
\text { Black/African } \\
\text { American: ONS: } \\
32 \text { (10.4\%); CG: } \\
35 \text { (11.2\%), } \\
\text { p=NS } \\
\text { White: ONS: } 273 \\
\text { (88.3\%); CG: } \\
267(85.3 \%), \\
\text { p=NS } \\
\text { Other: ONS: } 4 \\
\text { (1.3\%); CG: } 11 \\
\text { (3.5\%), p=NS } \\
\text { Government } \\
\text { sponsored } \\
\text { insurance, n (\%): } \\
\text { ONS: } 276(89 \%) ; \\
\text { CG: } 278(89 \%), \\
\text { p=NS }\end{array}$ & $\begin{array}{l}\text { SGA category, } \\
\text { n (\%): } \\
\text { SGA-B, mildly- } \\
\text { moderately } \\
\text { malnourished: } \\
\text { ONS: } 268(86.7 \%) \text {; } \\
\text { CG: } 275(87.9 \%) \text {, } \\
\text { p=NS } \\
\text { SGA-C, severely } \\
\text { malnourished: } \\
\text { ONS: } 41(13.3 \%) ; \\
\text { CG: } 38(12.1 \%) \text {, } \\
\text { p=NS } \\
\text { BMI in kg/m², } \\
\text { mean (SD): } \\
\text { ONS: } 24.3(5.2) ; \\
\text { CG: } 23.9(5.0), \\
\text { p=NS } \\
\text { Body weight in kg, } \\
\text { mean (SD): } \\
\text { ONS: } 67.5(17.4) ; \\
\text { CG: } 66.2(16.0), \\
\text { p=NS }\end{array}$ & $\begin{array}{l}\text { Primary } \\
\text { admission } \\
\text { diagnosis, } \mathrm{n}(\%) \text { : } \\
\text { AMI: ONS: } 25 \\
\text { (8.1\%); CG: } 30 \\
\text { (9.6\%), p=NS } \\
\text { COPD: ONS: } \\
\text { 105 (34.1\%); } \\
\text { CG: 109 } \\
\text { (34.8\%), p=NS } \\
\text { HF: ONS: } 78 \\
(25.3 \%) ; C G: 79 \\
\text { (25.2\%), p=NS } \\
\text { Pneumonia: } \\
\text { ONS: } 100 \\
\text { (32.5\%); CG: } 95 \\
\text { (30.4\%), p=NS } \\
\text { Charlson } \\
\text { comorbidity } \\
\text { index, mean } \\
\text { (SD): ONS: } 2.05 \\
\text { (1.46); CG: } 2.12 \\
\text { (1.48), p=NS }\end{array}$ \\
\hline
\end{tabular}




\begin{tabular}{|c|c|c|c|c|c|c|c|}
\hline $\begin{array}{l}\text { Author/Year } \\
\text { Study Design } \\
\text { Country/ } \\
\text { Setting }\end{array}$ & Objective & $\begin{array}{l}\text { Patient Inclusion/ } \\
\text { Exclusion Criteria }\end{array}$ & $\begin{array}{l}\text { Intervention } \\
\text { Control }\end{array}$ & $\begin{array}{l}\text { Number of } \\
\text { Patients per } \\
\text { Study Arm } \\
\text { Follow-up } \\
\text { Duration }\end{array}$ & $\begin{array}{l}\text { Age } \\
\text { Gender } \\
\text { Race/Ethnicity } \\
\text { Socioeconomic } \\
\text { Status (SES) }\end{array}$ & $\begin{array}{l}\text { Screening } \\
\text { Instrument } \\
\text { Nutritional Status } \\
\text { BMI } \\
\text { Weight }\end{array}$ & $\begin{array}{l}\text { Underlying } \\
\text { Condition } \\
\text { Disease } \\
\text { Severity }\end{array}$ \\
\hline & & & & & $\begin{array}{l}\text { Income } \\
<\$ 25,000 \text { per } \\
\text { year, n (\%): } \\
\text { Tx: } 154(49 \%) \\
\text { CG: } 130(42 \%) \\
\text { p=NS }\end{array}$ & & \\
\hline $\begin{array}{l}\text { Gazzotti et al. } \\
2003^{82} \\
\text { RCT } \\
\text { Belgium }\end{array}$ & $\begin{array}{l}\text { Evaluate whether } \\
\text { daily oral } \\
\text { supplementation } \\
\text { versus usual care } \\
\text { improve LOS for } \\
\text { malnourished } \\
\text { patients. }\end{array}$ & $\begin{array}{l}\text { Included patients aged } \\
75 \text { or older admitted } \\
\text { for acute conditions } \\
\text { between November } \\
1999 \text { and end of } \\
\text { March } 2000 \text { in the } \\
\text { geriatric ward of a } \\
\text { hospital. Patients were } \\
\text { required to have an } \\
\text { MNA score between } \\
17 \text { and } 23.5, \text { indicating } \\
\text { they were at-risk for } \\
\text { malnutrition. } \\
\text { Excluded patients with } \\
\text { a medical condition } \\
\text { preventing oral } \\
\text { feeding, end-of-life } \\
\text { patients, patients with } \\
\text { severe dementia (Mini } \\
\text { Mental Score -10) [20], } \\
\text { patients presenting } \\
\text { clinical signs of } \\
\text { dehydration or HF, } \\
\text { and those suffering } \\
\text { from diseases } \\
\text { requiring special } \\
\text { dietary treatment } \\
\text { (kidney or liver failure). }\end{array}$ & $\begin{array}{l}\text { Oral nutrition } \\
\text { supplement (ONS): } \\
\text { Patients received a } \\
\text { sweet or salty sip } \\
\text { feed twice daily, } \\
\text { totaling } 500 \text { kcal and } \\
21 \text { grams of protein } \\
\text { daily in addition to } \\
\text { usual care. } \\
\text { Control group (CG): } \\
\text { Usual care }\end{array}$ & $\begin{array}{l}\text { ONS: } 39 \\
\text { CG: } 41 \\
2 \text { months }\end{array}$ & $\begin{array}{l}\text { Age, mean (SD): } \\
\text { ONS: } 81.5(7.6) ; \\
\text { CG: } 78.8(6.1) \text {, } \\
p=0.09 \\
\text { Female gender, } \\
\text { n (\%): } \\
\text { ONS: } 28(72 \%) ; \\
\text { CG: } 33(80 \%) \\
p=0.20 \\
\text { Race/Ethnicity: } \\
\text { NR } \\
\text { SES: NR }\end{array}$ & $\begin{array}{l}\text { MNA score, mean } \\
\text { (SD): } \\
\text { ONS: } 19.9(2.0) ; \\
\text { CG: } 20.2(2.4), \\
p=0.62 \\
\text { BMI in } \mathrm{kg} / \mathrm{m}^{2}, \\
\text { mean (SD): } \\
\text { ONS: } 24.8(4.5) ; \\
\text { CG: } 26.9(5.4), \\
p=0.07 \\
\text { Weight in kg, mean } \\
\text { (SD): } \\
\text { ONS: } 61.7(13.0) ; \\
\text { CG: } 65.6(13.7), \\
p=0.20\end{array}$ & $\begin{array}{l}\text { Underlying } \\
\text { Condition: NR }\end{array}$ \\
\hline
\end{tabular}




\begin{tabular}{|c|c|c|c|c|c|c|c|}
\hline $\begin{array}{l}\text { Author/Year } \\
\text { Study Design } \\
\text { Country/ } \\
\text { Setting }\end{array}$ & Objective & $\begin{array}{l}\text { Patient Inclusion/ } \\
\text { Exclusion Criteria }\end{array}$ & $\begin{array}{l}\text { Intervention } \\
\text { Control }\end{array}$ & $\begin{array}{l}\text { Number of } \\
\text { Patients per } \\
\text { Study Arm } \\
\text { Follow-up } \\
\text { Duration }\end{array}$ & $\begin{array}{l}\text { Age } \\
\text { Gender } \\
\text { Race/Ethnicity } \\
\text { Socioeconomic } \\
\text { Status (SES) }\end{array}$ & $\begin{array}{l}\text { Screening } \\
\text { Instrument } \\
\text { Nutritional Status } \\
\text { BMI } \\
\text { Weight }\end{array}$ & $\begin{array}{l}\text { Underlying } \\
\text { Condition } \\
\text { Disease } \\
\text { Severity }\end{array}$ \\
\hline $\begin{array}{l}\text { Ha et al. } \\
2010^{83} \\
\text { Norway }\end{array}$ & $\begin{array}{l}\text { Examine the effect } \\
\text { of individualized, } \\
\text { nutritional support } \\
\text { on weight loss, } \\
\text { functional outcomes, } \\
\text { and other clinical } \\
\text { outcomes. }\end{array}$ & $\begin{array}{l}\text { Included patients aged } \\
\text { over } 65 \text { between May } \\
2005 \text { and December } \\
2007 \text { admitted with a } \\
\text { stroke to a medical } \\
\text { acute ward in Norway. } \\
\text { All patients were at } \\
\text { nutritional risk or } \\
\text { undernourished } \\
\text { according to a MUST } \\
\text { score >0. } \\
\text { Excluded patients with } \\
\text { a stroke diagnosis that } \\
\text { could not be } \\
\text { confirmed, was } \\
\text { critically ill, had severe } \\
\text { dementia, could not be } \\
\text { weighed, or if there } \\
\text { was a planned } \\
\text { discharged within } \\
24 \text { hours after the first } \\
\text { visit by the trial } \\
\text { assessor. }\end{array}$ & $\begin{array}{l}\text { Intervention (Tx): } \\
\text { Individualized, } \\
\text { nutrition treatment } \\
\text { with a goal to } \\
\text { maintain or improve } \\
\text { nutritional status } \\
\text { according to } \\
\text { individual intake and } \\
\text { needs. } \\
\text { Control (CG): } \\
\text { Routine care }\end{array}$ & $\begin{array}{l}\text { Tx: } 84 \\
\text { CG: } 86 \\
3 \text { months }\end{array}$ & $\begin{array}{l}\text { Age, mean (SD): } \\
\text { Tx: } 78.5(7.4) ; \\
\text { CG: } 79.7(6.8) \\
p=0.34 \\
\text { Female gender, } \\
n(\%): \\
\text { Tx: } 33(57 \%) ; \\
\text { CG: } 31(47 \%) \\
p=0.27 \\
\text { Race/Ethnicity: } \\
\text { NR } \\
\text { SES: NR }\end{array}$ & $\begin{array}{l}\text { MUST } \\
\text { Classification of } \\
\text { Undernourished, } \\
n \text { (\%): } \\
\text { Tx: } 5(8.6 \%) ; \text { CG: } 3 \\
(4.5 \%), p=0.47 \\
\text { BMI in kg/m², } \\
\text { mean (SD): } \\
\text { Tx: } 24.5(4.0) \text {; CG: } \\
26.2(4.6), p=0.031 \\
\text { Weight in kg, mean } \\
\text { (SD): } \\
\text { Tx: } 66.3(13.6) ; \\
\text { CG: } 72.6(14.8), \\
\text { p=0.015 }\end{array}$ & Diagnosis: NR \\
\hline $\begin{array}{l}\text { Holyday et al. } \\
2011^{84} \\
\text { RCT } \\
\text { Australia }\end{array}$ & $\begin{array}{l}\text { Assess the impact } \\
\text { of nutrition } \\
\text { screening and } \\
\text { malnutrition care } \\
\text { plan intervention on } \\
\text { patient outcomes } \\
\text { and health costs. }\end{array}$ & $\begin{array}{l}\text { Included patients } \\
\text { transferred from the } \\
\text { emergency } \\
\text { department to an } \\
\text { Australian hospital } \\
\text { between April and } \\
\text { September } 2006 . \\
\text { Patients were required } \\
\text { to have an MNA score } \\
\text { greater than } 17, \text { with } \\
\text { scores between } 17 \\
\text { and } 23.5 \text { indicating at- } \\
\text { risk for malnutrition, } \\
\text { and scores less than }\end{array}$ & $\begin{array}{l}\text { Intervention (Tx): } \\
\text { Malnutrition care } \\
\text { plan involving the } \\
\text { modification of } \\
\text { hospital meals } \\
\text { (texture modification } \\
\text { and fortification), } \\
\text { prescription of } \\
\text { nutrition } \\
\text { supplements, } \\
\text { flagging for } \\
\text { assistance with } \\
\text { meals by ward } \\
\text { based staff, and }\end{array}$ & $\begin{array}{l}\text { At-Risk } \\
\text { Tx: } 47 \\
\text { CG: } 40 \\
6 \text { months } \\
\text { Malnourished } \\
\text { Tx: } 12 \\
\text { CG: } 20 \\
6 \text { months }\end{array}$ & $\begin{array}{l}\underline{\text { At-Risk }} \\
\text { Age, mean (SE): } \\
\text { Tx: } 83.2(0.9) ; \\
\text { CG: } 83.1(1.3), \\
\text { p=0.98 } \\
\text { Female gender, } \\
\text { n (\%): } \\
\text { Tx: } 23(57.5 \%) ; \\
\text { CG: } 32(68.1 \%), \\
\text { p=NR } \\
\text { Race/Ethnicity: } \\
\text { NR }\end{array}$ & $\begin{array}{l}\frac{\text { At-Risk }}{\text { MNA score, mean }} \\
(\mathrm{SE}): \\
\text { Tx: } 20.0(0.3) \text {; CG: } \\
19.8(0.3), \mathrm{p}=0.64 \\
\text { BMl in } \mathrm{kg} / \mathrm{m}^{2} \text {, } \\
\text { mean }(\mathrm{SE}): \\
\text { Tx: } 23.0(0.8) \text {; CG: } \\
24.5(0.9), \mathrm{p}=0.23 \\
\text { Weight in kg, mean } \\
\text { (SE): }\end{array}$ & $\begin{array}{l}\text { At-Risk } \\
\text { Charlson } \\
\text { comorbidity } \\
\text { index, mean } \\
\text { (SE): } \\
\text { Tx: } 5.8(0.2) ; \\
\text { CG: } 5.4(0.2) \text {, } \\
\text { p=0.26 } \\
\text { Malnourished } \\
\text { Charlson } \\
\text { comorbidity }\end{array}$ \\
\hline
\end{tabular}




\begin{tabular}{|c|c|c|c|c|c|c|c|}
\hline $\begin{array}{l}\text { Author/Year } \\
\text { Study Design } \\
\text { Country/ } \\
\text { Setting }\end{array}$ & Objective & $\begin{array}{l}\text { Patient Inclusion/ } \\
\text { Exclusion Criteria }\end{array}$ & $\begin{array}{l}\text { Intervention } \\
\text { Control }\end{array}$ & $\begin{array}{l}\text { Number of } \\
\text { Patients per } \\
\text { Study Arm } \\
\text { Follow-up } \\
\text { Duration }\end{array}$ & $\begin{array}{l}\text { Age } \\
\text { Gender } \\
\text { Race/Ethnicity } \\
\text { Socioeconomic } \\
\text { Status (SES) }\end{array}$ & $\begin{array}{l}\text { Screening } \\
\text { Instrument } \\
\text { Nutritional Status } \\
\text { BMI } \\
\text { Weight }\end{array}$ & $\begin{array}{l}\text { Underlying } \\
\text { Condition } \\
\text { Disease } \\
\text { Severity }\end{array}$ \\
\hline & & $\begin{array}{l}17 \text { indicating } \\
\text { malnourished. } \\
\text { Excluded patients with } \\
\text { an expected LOS less } \\
\text { than } 72 \text { hours, } \\
\text { palliative (not for } \\
\text { active treatment) or } \\
\text { who were unable to be } \\
\text { nutritionally assessed } \\
\text { (non-English speaking, } \\
\text { severe dementia/ } \\
\text { confusion, non- } \\
\text { cooperative/refused), } \\
\text { already seen by a } \\
\text { dietitian during the } \\
\text { admission, or enrolled } \\
\text { in the study during a } \\
\text { previous admission. }\end{array}$ & $\begin{array}{l}\text { education of } \\
\text { patients and their } \\
\text { carers regarding } \\
\text { optimization of } \\
\text { nutrition intake and } \\
\text { referral to other } \\
\text { health professionals } \\
\text { for discharge } \\
\text { planning. } \\
\text { Control (CG): Usual } \\
\text { nutrition care }\end{array}$ & & $\begin{array}{l}\text { Socioeconomic } \\
\text { status: NR } \\
\text { Malnourished } \\
\text { Age, mean (SE): } \\
\text { Tx: } 86.9(2.3) ; \\
\text { CG: } 84.4(1.1), \\
\text { p=0.27 } \\
\text { Female gender, } \\
\text { n (\%): } \\
\text { Tx: } 11(55.0 \%) ; \\
\text { CG: } 3(25.0 \%), \\
\text { p=NR } \\
\text { Race/Ethnicity: } \\
\text { NR } \\
\text { SES: NR }\end{array}$ & $\begin{array}{l}\text { Tx: } 60.2 \text { (2.4); CG: } \\
65.0(2.6), p=0.18 \\
\text { Malnourished } \\
\text { MNA score, mean } \\
\text { (SE): } \\
\text { Tx: } 12.7(0.7) \text {; CG: } \\
\text { 13.2 }(0.6), p=0.59 \\
\text { BMI in kg/m², } \\
\text { mean (SE): } \\
\text { Tx: } 21.2(1.5) \text {; CG: } \\
\text { 19.0 (1.2), p=0.28 } \\
\text { Weight in kg, mean } \\
\text { (SE): } \\
\text { Tx: } 59.7(5.4) ; \text { CG: } \\
\text { 52.6 (3.9), p=29 }\end{array}$ & $\begin{array}{l}\text { index, mean } \\
\text { (SE): } \\
\text { Tx: } 5.7(0.4) ; \\
\text { CG: } 5.4(0.2) \text {, } \\
p=0.50\end{array}$ \\
\hline $\begin{array}{l}\text { Johansen } \\
\text { et al. } 2004^{85} \\
\text { RCT } \\
\text { Denmark }\end{array}$ & $\begin{array}{l}\text { To evaluate the } \\
\text { effect of a } \\
\text { specialized } \\
\text { nutritional team } \\
\text { versus standard } \\
\text { regime on LOS, } \\
\text { complications, and } \\
\text { quality of life in a } \\
\text { random sample of } \\
\text { hospitalized patients } \\
\text { at nutritional risk. }\end{array}$ & $\begin{array}{l}\text { Included patients were } \\
\text { admitted to one of } \\
\text { three participating } \\
\text { Danish hospitals and } \\
\text { screened at nutritional } \\
\text { risk according to the } \\
\text { NRS-2002 (total score } \\
\geq 3 \text { ). } \\
\text { Excluded patients had } \\
\text { less than } 4 \text { days' } \\
\text { expected admissions, } \\
\text { less than } 18 \text { years of } \\
\text { age, less than } 1 \text { month } \\
\text { of expected survival, } \\
\text { did not understand the } \\
\text { Danish language, } \\
\text { previously participated } \\
\text { in nutrition trials, were } \\
\text { placed next to another }\end{array}$ & $\begin{array}{l}\text { Intervention (Tx): } \\
\text { Nutritional care with } \\
\text { motivation of patient } \\
\text { and staff, } \\
\text { adjustment of the } \\
\text { nutritional plan by } \\
\text { estimation of } \\
\text { protein- and energy } \\
\text { requirements and } \\
\text { ordering food in } \\
\text { collaboration with } \\
\text { the patient and } \\
\text { securement of the } \\
\text { supply of food } \\
\text { ordered. } \\
\text { Control (CG): } \\
\text { Standard regime of } \\
\text { nutritional care }\end{array}$ & $\begin{array}{l}\text { Tx: } 108 \\
\text { CG: } 104 \\
28 \text { days }\end{array}$ & $\begin{array}{l}\text { Age, mean (SE): } \\
\text { Tx: } 62.0(1.6) ; \\
\text { CG: } 62.4(1.7) \text {, } \\
\text { p=NR } \\
\text { Female gender, } \\
\text { n (\%): } \\
\text { Tx: } 54(50 \%) \\
\text { CG: } 56(54 \%) \\
\text { p=NR } \\
\text { Race/Ethnicity: } \\
\text { NR } \\
\text { SES: NR }\end{array}$ & $\begin{array}{l}\text { NRS-2002 score } \\
\text { for nutritional } \\
\text { status, mean (SE): } \\
\text { Tx: } 2.4(0.08) \text {; CG: } \\
2.5(0.07), p=N R \\
\text { NRS-2002 score } \\
\text { for severity of } \\
\text { disease, mean } \\
\text { (SE): } \\
\text { Tx: } 1.0(0.07) \text {; CG: } \\
1.0(0.06), p=N R \\
\text { BMI in } \mathrm{kg} / \mathrm{m}^{2}, \\
\text { mean }(\mathrm{SE}): \\
\text { Tx: } 21.2(0.50) ; \\
\text { CG: } 21.8(0.48), \\
\text { p=NR }\end{array}$ & $\begin{array}{l}\text { Primary } \\
\text { admission } \\
\text { diagnosis, } \mathrm{n}(\%) \text { : } \\
\text { Cardiovascular } \\
\text { disease: Tx: } 7 \\
(6.5 \%) \text {; CG: } 4 \\
(3.8 \%), p=N R \\
\text { GI disorders: } \\
\text { Tx: } 13(12.0 \%) \text {; } \\
\text { CG: } 14(13.4 \%) \text {, } \\
\text { p=NR } \\
\text { Neurological } \\
\text { disorders: Tx: } 6 \\
\text { (5.6\%); CG: } 6 \\
\text { (5.8\%), p=NR } \\
\text { Respiratory } \\
\text { disease: Tx: } 14\end{array}$ \\
\hline
\end{tabular}




\begin{tabular}{|c|c|c|c|c|c|c|c|}
\hline $\begin{array}{l}\text { Author/Year } \\
\text { Study Design } \\
\text { Country/ } \\
\text { Setting }\end{array}$ & Objective & $\begin{array}{l}\text { Patient Inclusion/ } \\
\text { Exclusion Criteria }\end{array}$ & $\begin{array}{l}\text { Intervention } \\
\text { Control }\end{array}$ & $\begin{array}{l}\text { Number of } \\
\text { Patients per } \\
\text { Study Arm } \\
\text { Follow-up } \\
\text { Duration }\end{array}$ & $\begin{array}{l}\text { Age } \\
\text { Gender } \\
\text { Race/Ethnicity } \\
\text { Socioeconomic } \\
\text { Status (SES) }\end{array}$ & $\begin{array}{l}\text { Screening } \\
\text { Instrument } \\
\text { Nutritional Status } \\
\text { BMI } \\
\text { Weight }\end{array}$ & $\begin{array}{l}\text { Underlying } \\
\text { Condition } \\
\text { Disease } \\
\text { Severity }\end{array}$ \\
\hline & & $\begin{array}{l}\text { participant in the same } \\
\text { room, pregnant or } \\
\text { lactating healthy } \\
\text { women, had } \\
\text { psychiatric disorders, } \\
\text { were receiving } \\
\text { hemodialysis, and who } \\
\text { were already } \\
\text { receiving, or were } \\
\text { planned to receive, a } \\
\text { standard parenteral or } \\
\text { PEG-tube feeding. }\end{array}$ & & & & $\begin{array}{l}\text { Weight in kg, mean } \\
\text { (SE): } \\
\text { Tx: } 61.5(1.65) ; \\
\text { CG: } 62.4(1.51) \\
\text { p=NR }\end{array}$ & $\begin{array}{l}\text { (13.0\%); CG: } 19 \\
(18.3 \%), p=N R \\
\text { Internal } \\
\text { medicine, other: } \\
\text { Tx: } 34(31.5 \%) ; \\
\text { CG: } 27(26.0 \%) \text {, } \\
\text { p=NR } \\
\text { Surgical, other: } \\
\text { Tx: } 34(31.4 \%) ; \\
\text { CG: } 34(32.7 \%) \text {, } \\
\text { p=NR }\end{array}$ \\
\hline $\begin{array}{l}\text { Munk et al. } \\
2014^{86} \\
\text { RCT } \\
\text { Denmark, } \\
\text { Sweden }\end{array}$ & $\begin{array}{l}\text { Investigate whether } \\
\text { a novel food service } \\
\text { concept with } \\
\text { protein- } \\
\text { supplementation in } \\
\text { addition to standard } \\
\text { food service would } \\
\text { increase protein } \\
\text { intake, increase } \\
\text { energy intake, and } \\
\text { impact LOS in }\end{array}$ & $\begin{array}{l}\text { Included patients } \\
\text { admitted to a Denmark } \\
\text { hospital between } \\
\text { October } 2011 \text { to } \\
\text { February } 2012 \text { aged } \\
18 \text { years or older, at } \\
\text { nutritional risk } \\
\text { (NRS-2002 score } \geq 3 \text { ), } \\
\text { able to eat orally, had } \\
\text { an anticipated length } \\
\text { of hospitalization }\end{array}$ & $\begin{array}{l}\text { Intervention (Tx): } \\
\text { A targeted food } \\
\text { concept consisting } \\
\text { of an a la carte } \\
\text { menu of small } \\
\text { dishes enriched with } \\
\text { natural energy- } \\
\text { dense ingredients } \\
\text { and supplemented } \\
\text { with a high-quality } \\
\text { protein powder in }\end{array}$ & $\begin{array}{l}\text { Tx: } 41 \\
\text { CG: } 40 \\
7 \text { days }\end{array}$ & $\begin{array}{l}\text { Age, mean (SD): } \\
\text { Tx: } 75(10) ; \text { CG: } \\
74(11), p=N R \\
\text { Female gender, } \\
\text { n (\%): } \\
\text { Tx: } 25(61.0 \%) ; \\
\text { CG: } 22(55.0 \%) \text {, } \\
\text { p=NR } \\
\text { Race/Ethnicity: } \\
\text { NR }\end{array}$ & $\begin{array}{l}\text { NRS-2002 total } \\
\text { score, mean (SD): } \\
\text { Tx: } 3.88(0.95) ; \\
\text { CG: } 3.43(0.68) \text {, } \\
\text { p=NR } \\
\text { BMI in } \mathrm{kg} / \mathrm{m}^{2} \text {, } \\
\text { mean (SD): } \\
\text { Tx: } 21(4) ; \text { CG: } 22 \\
\text { (4), p=NR }\end{array}$ & $\begin{array}{l}\text { Admitting } \\
\text { department, } \\
\text { n (\%): } \\
\text { Oncology: Tx: } 14 \\
\text { (34.1\%); CG: } 15 \\
(37.5 \%), p=N R \\
\text { Orthopedic } \\
\text { surgery: Tx: } 12 \\
(29.3 \%) ; \text { CG: } 10 \\
(25.0 \%), p=N R\end{array}$ \\
\hline & $\begin{array}{l}\text { hospitalized patients } \\
\text { at nutritional risk. }\end{array}$ & $\begin{array}{l}\geq 3 \text { days, and had } \\
\text { sufficient language } \\
\text { proficiency. } \\
\text { Excluded patients with } \\
\text { dysphagia, food } \\
\text { allergy or intolerance, } \\
\text { anatomical } \\
\text { obstructions } \\
\text { preventing oral food } \\
\text { intake, patients who } \\
\text { exclusively received } \\
\text { enteral or parenteral } \\
\text { nutrition, and } \\
\text { terminally ill. }\end{array}$ & $\begin{array}{l}\text { addition to standard } \\
\text { hospital food } \\
\text { service. } \\
\text { Control Group (CG): } \\
\text { Standard hospital } \\
\text { food service (buffet } \\
\text { style serving system } \\
\text { with } 3 \text { main meals } \\
\text { and } 2-3 \text { in-between } \\
\text { snacks or ONS) }\end{array}$ & & $\begin{array}{l}\text { Socioeconomic } \\
\text { status: NR }\end{array}$ & $\begin{array}{l}\text { (SD): } \\
\text { Tx: } 60 \text { (14); CG: } 65 \\
\text { (13), p=NR }\end{array}$ & $\begin{array}{l}\text { Urology: Tx: } 15 \\
(36.6 \%) ; \mathrm{CG}: 15 \\
(37.5 \%), p=\mathrm{NR}\end{array}$ \\
\hline
\end{tabular}




\begin{tabular}{|c|c|c|c|c|c|c|c|}
\hline $\begin{array}{l}\text { Author/Year } \\
\text { Study Design } \\
\text { Country/ } \\
\text { Setting }\end{array}$ & Objective & $\begin{array}{l}\text { Patient Inclusion/ } \\
\text { Exclusion Criteria }\end{array}$ & $\begin{array}{l}\text { Intervention } \\
\text { Control }\end{array}$ & $\begin{array}{l}\text { Number of } \\
\text { Patients per } \\
\text { Study Arm } \\
\text { Follow-up } \\
\text { Duration }\end{array}$ & $\begin{array}{l}\text { Age } \\
\text { Gender } \\
\text { Race/Ethnicity } \\
\text { Socioeconomic } \\
\text { Status (SES) }\end{array}$ & $\begin{array}{l}\text { Screening } \\
\text { Instrument } \\
\text { Nutritional Status } \\
\text { BMI } \\
\text { Weight }\end{array}$ & $\begin{array}{l}\text { Underlying } \\
\text { Condition } \\
\text { Disease } \\
\text { Severity }\end{array}$ \\
\hline $\begin{array}{l}\text { Rufenacht } \\
\text { et al. } 2011^{87} \\
\text { RCT } \\
\text { Switzerland }\end{array}$ & $\begin{array}{l}\text { Study the impact of } \\
\text { nutritional therapy } \\
\text { on quality of life and } \\
\text { food intake. }\end{array}$ & $\begin{array}{l}\text { Included patients from } \\
\text { April } 2005 \text { to March } \\
2006 \text { admitted with an } \\
\text { NRS-2002 score } \geq 3 \text {, } \\
\text { estimated LOS } \\
\geq 10 \text { days, unintended } \\
\text { loss of body weight } \\
\geq 5 \% \text { of usual weight } \\
\text { over the previous } \\
2 \text { months, loss of } \\
\text { appetite, and given } \\
\text { informed consent to } \\
\text { participate. } \\
\text { Excluded people with } \\
\text { terminal illness, } \\
\text { existing enteral or } \\
\text { parenteral nutrition, } \\
\text { ongoing nutritional } \\
\text { counseling or } \\
\text { interventions, e.g., } \\
\text { intake of ONSs, } \\
\text { impaired cognition, } \\
\text { and incapability to give } \\
\text { consent. }\end{array}$ & $\begin{array}{l}\text { Nutrition therapy } \\
\text { (Tx): Patients } \\
\text { received individual } \\
\text { nutritional } \\
\text { counseling and } \\
\text { interventions, } \\
\text { including oral } \\
\text { nutritional } \\
\text { supplements, if } \\
\text { appropriate. } \\
\text { Oral nutrition } \\
\text { supplementation } \\
\text { group (ONS): Oral } \\
\text { nutritional } \\
\text { supplements } \\
\text { provided in addition } \\
\text { to hospital meals } \\
\text { without further } \\
\text { instruction or } \\
\text { counseling. }\end{array}$ & $\begin{array}{l}\text { Tx: } 18 \\
\text { ONS: } 18 \\
2 \text { months }\end{array}$ & $\begin{array}{l}\text { Age, mean (SD): } \\
\text { Tx: } 69.2(12.6) ; \\
\text { ONS: } 70.8 \\
\text { (13.3), p=0.669 } \\
\text { Female gender, } \\
\text { n (\%): } \\
\text { Tx: } 7(39 \%) ; \\
\text { ONS: } 9(50 \%) \\
\text { p=NR } \\
\text { Race/Ethnicity: } \\
\text { NR } \\
\text { SES: NR }\end{array}$ & $\begin{array}{l}\text { NRS-2002 score, } \\
\text { mean (SD): } \\
\text { Tx: } 3.8(0.7) \text {; ONS: } \\
3.9 \text { (0.9), p=0.657 } \\
\text { SGA score, n (\%): } \\
\text { SGA-A, well- } \\
\text { nourished: } \\
\text { Tx: } 2 \text { (11\%); ONS: } \\
1 \text { (6\%), p=NR } \\
\text { SGA-B, mildly- } \\
\text { moderately } \\
\text { malnourished: } \\
\text { Tx: } 16 \text { (89\%); } \\
\text { ONS: } 17 \text { (94\%), } \\
\text { p=NR } \\
\text { SGA-C, severely } \\
\text { malnourished: } \\
\text { Tx: } 0 \text { (0\%); ONS: } 0 \\
\text { (0\%), p=NR } \\
\text { BMI in kg, mean } \\
\text { (SD): } \\
\text { Tx: } 22 \text { (4); ONS: } 22 \\
\text { (3.6), p=0.950 } \\
\text { Weight in kg, mean } \\
\text { (SD): } \\
\text { Tx: } 64.6(16.3) ; \\
\text { ONS: } 57.8 \text { (11.4), } \\
\text { p=0.304 }\end{array}$ & $\begin{array}{l}\text { Primary } \\
\text { admission } \\
\text { diagnosis, } n(\%) \text { : } \\
\text { COPD: Tx: } 2 \\
\text { (11\%); ONS: } 2 \\
(11 \%), p=N R \\
\text { Malignant } \\
\text { tumors: Tx: } 7 \\
\text { (39\%); ONS: } 7 \\
(39 \%), p=N R \\
\text { Pneumonia: Tx: } \\
3(17 \%) ; \text { ONS: } 4 \\
(22 \%), p=N R \\
\text { Other: Tx: } 6 \\
(33 \%) ; \text { ONS: } 5 \\
(28 \%), p=N R \\
\text { Case mix index, } \\
\text { mean (SD): } \\
\text { Tx: 1.453 } \\
\text { (0.953); ONS: } \\
\text { 1.451 (0.950), } \\
\text { p=NR }\end{array}$ \\
\hline
\end{tabular}




\begin{tabular}{|c|c|c|c|c|c|c|c|}
\hline $\begin{array}{l}\text { Author/Year } \\
\text { Study Design } \\
\text { Country/ } \\
\text { Setting }\end{array}$ & Objective & $\begin{array}{l}\text { Patient Inclusion/ } \\
\text { Exclusion Criteria }\end{array}$ & $\begin{array}{l}\text { Intervention } \\
\text { Control }\end{array}$ & $\begin{array}{l}\text { Number of } \\
\text { Patients per } \\
\text { Study Arm } \\
\text { Follow-up } \\
\text { Duration }\end{array}$ & $\begin{array}{l}\text { Age } \\
\text { Gender } \\
\text { Race/Ethnicity } \\
\text { Socioeconomic } \\
\text { Status (SES) }\end{array}$ & $\begin{array}{l}\text { Screening } \\
\text { Instrument } \\
\text { Nutritional Status } \\
\text { BMI } \\
\text { Weight }\end{array}$ & $\begin{array}{l}\text { Underlying } \\
\text { Condition } \\
\text { Disease } \\
\text { Severity }\end{array}$ \\
\hline $\begin{array}{l}\text { Schuetz et al. } \\
2019^{88} \\
\text { RCT } \\
\text { Switzerland }\end{array}$ & $\begin{array}{l}\text { Evaluate if protocol- } \\
\text { guided } \\
\text { individualized } \\
\text { nutritional support to } \\
\text { reach protein and } \\
\text { caloric goals } \\
\text { reduces the risk of } \\
\text { adverse clinical } \\
\text { outcomes in medical } \\
\text { inpatients at } \\
\text { nutritional risk. }\end{array}$ & $\begin{array}{l}\text { Included patients } \\
\text { between April } 1,2014 \\
\text { and February 28, } 2018 \\
\text { admitted to one of } \\
\text { eight Swiss secondary } \\
\text { or tertiary care } \\
\text { hospitals aged at least } \\
18 \text { years with an NRS- } \\
2002 \text { score } \geq 3 \text { and } \\
\text { expected LOS of more } \\
\text { than } 4 \text { days. All } \\
\text { patients were required } \\
\text { to provide informed } \\
\text { consent within } \\
48 \text { hours of hospital } \\
\text { admission. } \\
\text { Excluded patients who } \\
\text { were initially admitted } \\
\text { to intensive care units } \\
\text { or surgical units; } \\
\text { unable to ingest oral } \\
\text { nutrition; already } \\
\text { receiving nutritional } \\
\text { support on admission; } \\
\text { with a terminal } \\
\text { condition; admitted to } \\
\text { hospital because of } \\
\text { anorexia nervosa, } \\
\text { acute pancreatitis, } \\
\text { acute liver failure, } \\
\text { cystic fibrosis, or stem- } \\
\text { cell transplantation; } \\
\text { after gastric bypass } \\
\text { surgery; with } \\
\text { contraindications for } \\
\text { nutritional support; and }\end{array}$ & $\begin{array}{l}\text { Intervention (Tx): } \\
\text { Nutritional support } \\
\text { including } \\
\text { establishing } \\
\text { individual nutrition } \\
\text { targets and creating } \\
\text { a strategy to reach } \\
\text { nutrition targets } \\
\text { within } 48 \text { hours of } \\
\text { hospital admission. } \\
\text { Control Group (CG): } \\
\text { Standard hospital } \\
\text { food }\end{array}$ & $\begin{array}{l}\text { Tx: } 1,015 \\
\text { CG: } 1,013 \\
30 \text { days }\end{array}$ & $\begin{array}{l}\text { Age, mean (SD): } \\
\text { Tx: } 72.4(14.1) ; \\
\text { CG: } 72.8(14.1) \text {, } \\
\text { p=0.56 } \\
\text { Female gender, } \\
\text { n (\%): } \\
\text { Tx: } 490(48 \%) ; \\
\text { CG: } 474(47 \%) \text {, } \\
\text { p=0.50 } \\
\text { Race/Ethnicity: } \\
\text { NR } \\
\text { SES: NR }\end{array}$ & $\begin{array}{l}\text { NRS-2002 score, } \\
\text { mean (SD): } \\
\text { Tx: } 4.05(0.87) ; \\
\text { CG: } 4.05(0.88) \text {, } \\
\text { p=0.0.98 } \\
\text { BMI in } \mathrm{kg} / \mathrm{m}^{2} \text {, } \\
\text { mean (SD): } \\
\text { Tx: } 24.9(5.4) ; \text { CG: } \\
24.7(5.3), p=0.43 \\
\text { Weight in kg, mean } \\
(\mathrm{SD}): \\
\text { Tx: } 70.9(16.4) ; \\
\text { CG: } 70.9(16.4), \\
p=0.91\end{array}$ & 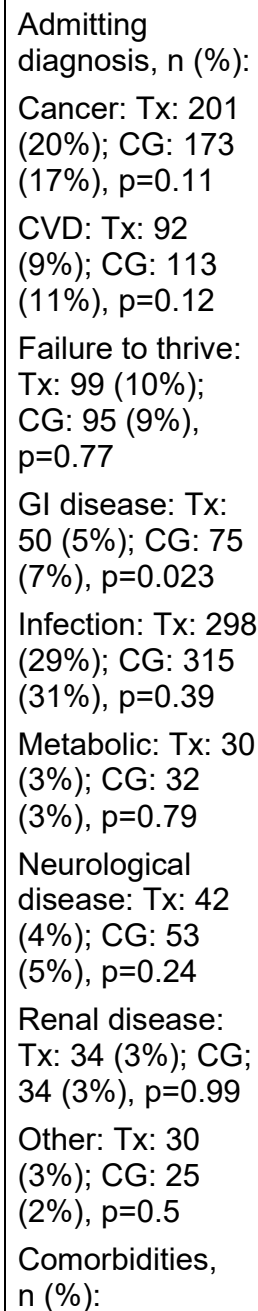 \\
\hline
\end{tabular}




\begin{tabular}{|c|c|c|c|c|c|c|c|}
\hline $\begin{array}{l}\text { Author/Year } \\
\text { Study Design } \\
\text { Countryl } \\
\text { Setting }\end{array}$ & Objective & $\begin{array}{l}\text { Patient Inclusion/ } \\
\text { Exclusion Criteria }\end{array}$ & $\begin{array}{l}\text { Intervention } \\
\text { Control }\end{array}$ & $\begin{array}{l}\text { Number of } \\
\text { Patients per } \\
\text { Study Arm } \\
\text { Follow-up } \\
\text { Duration }\end{array}$ & $\begin{array}{l}\text { Age } \\
\text { Gender } \\
\text { Race/Ethnicity } \\
\text { Socioeconomic } \\
\text { Status (SES) } \\
\end{array}$ & $\begin{array}{l}\text { Screening } \\
\text { Instrument } \\
\text { Nutritional Status } \\
\text { BMI } \\
\text { Weight } \\
\end{array}$ & $\begin{array}{l}\text { Underlying } \\
\text { Condition } \\
\text { Disease } \\
\text { Severity }\end{array}$ \\
\hline & & $\begin{array}{l}\text { previously included in } \\
\text { the trial. }\end{array}$ & & & & & $\begin{array}{l}\text { CHD: Tx: } 287 \\
\text { (28\%); CG: } 279 \\
(28 \%), p=0.71 \\
\text { CHF: Tx: } 174 \\
\text { (17\%); CG: } 179 \\
(18 \%), p=0.75 \\
\text { CKD: Tx: } 323 \\
\text { (32\%); CG: } 318 \\
(31 \%), p=0.83 \\
\text { COPD: Tx: } 147 \\
\text { (14\%); CG: } 156 \\
\text { (15\%), p=0.56 } \\
\text { CVA: Tx: } 75 \\
\text { (7\%); CG: } 87 \\
\text { (9\%), p=0.32 } \\
\text { Dementia: Tx: } 39 \\
\text { (4\%); CG: } 36 \\
(4 \%), p=0.73 \\
\text { Diabetes: } \\
\text { Tx: } 215 \text { (21\%); } \\
\text { CG: } 213(21 \%), \\
\text { p=0.93 } \\
\text { HTN: Tx: } 557 \\
\text { (55\%); CG: } 552 \\
(54 \%), p=0.86 \\
\text { Malignant } \\
\text { disease: Tx: } 338 \\
\text { (33\%); CG: } 329 \\
\text { (32\%), p=0.69 } \\
\text { Peripheral } \\
\text { disease: Tx: } 80 \\
\text { (8\%); CG: } 106 \\
\text { (10\%), p=0.044 }\end{array}$ \\
\hline
\end{tabular}




\begin{tabular}{|c|c|c|c|c|c|c|c|}
\hline $\begin{array}{l}\text { Author/Year } \\
\text { Study Design } \\
\text { Country/ } \\
\text { Setting }\end{array}$ & Objective & $\begin{array}{l}\text { Patient Inclusion/ } \\
\text { Exclusion Criteria }\end{array}$ & $\begin{array}{l}\text { Intervention } \\
\text { Control }\end{array}$ & $\begin{array}{l}\text { Number of } \\
\text { Patients per } \\
\text { Study Arm } \\
\text { Follow-up } \\
\text { Duration }\end{array}$ & $\begin{array}{l}\text { Age } \\
\text { Gender } \\
\text { Race/Ethnicity } \\
\text { Socioeconomic } \\
\text { Status (SES) }\end{array}$ & $\begin{array}{l}\text { Screening } \\
\text { Instrument } \\
\text { Nutritional Status } \\
\text { BMI } \\
\text { Weight }\end{array}$ & $\begin{array}{l}\text { Underlying } \\
\text { Condition } \\
\text { Disease } \\
\text { Severity }\end{array}$ \\
\hline $\begin{array}{l}\text { Sharma et al. } \\
2017^{89} \\
\text { RCT } \\
\text { Australia }\end{array}$ & $\begin{array}{l}\text { Examine the } \\
\text { efficacy of providing } \\
\text { an early nutrition } \\
\text { intervention and its } \\
\text { continuation post- } \\
\text { discharge in older } \\
\text { hospitalized } \\
\text { patients. }\end{array}$ & $\begin{array}{l}\text { Include patients } \geq 60 \\
\text { admitted to the } \\
\text { general medicine } \\
\text { department of an } \\
\text { Australian medical } \\
\text { center between } \\
\text { November } 2014 \text { and } \\
\text { June } 2016 \text { and } \\
\text { confirmed as } \\
\text { malnourished by PG- } \\
\text { SGA classes B and C. } \\
\text { Exclude patients } \\
\text { receiving palliative } \\
\text { care, patients residing } \\
\text { in rural areas, } \\
\text { Indigenous Australians } \\
\text { and non-English } \\
\text { speaking patients and } \\
\text { patients unable to give } \\
\text { informed consent. } \\
\text { Rural patients were } \\
\text { excluded due to } \\
\text { inadequate funds to } \\
\text { travel to rural areas to } \\
\text { follow up these } \\
\text { participants and } \\
\text { Indigenous Australians } \\
\text { and non-English } \\
\text { speaking subjects } \\
\text { were excluded due to } \\
\text { lack of funds to seek } \\
\text { services of an } \\
\text { Aborigine's Liaison } \\
\text { Officer/interpreter. }\end{array}$ & $\begin{array}{l}\text { Intervention (Tx): } \\
\text { Combination of } \\
\text { strategies including } \\
\text { oral nutrition } \\
\text { supplements } \\
(1-2.2 \mathrm{kcal} / \mathrm{ml} \text { and } \\
0.05-0.12 \mathrm{~g} \text { of } \\
\text { protein } / \mathrm{ml}) \text {, mid- } \\
\text { meal snacks and } \\
\text { food fortification } \\
\text { were employed with } \\
\text { consideration given } \\
\text { to individual } \\
\text { patients' food } \\
\text { preferences and } \\
\text { dietetic counseling } \\
\text { was provided to the } \\
\text { patients and care } \\
\text { providers to } \\
\text { augment energy } \\
\text { intake including } \\
\text { flagging for } \\
\text { assistance with } \\
\text { meals by ward } \\
\text { based staff if } \\
\text { needed. } \\
\text { Control Group (CG): } \\
\text { Usual care }\end{array}$ & $\begin{array}{l}\text { Tx: } 78 \\
\text { CG: } 70 \\
3 \text { months }\end{array}$ & $\begin{array}{l}\text { Age, mean } \\
\text { (95\% Cl): } \\
\text { Tx: } 82.0(80.0 \text { to } \\
83.9) ; \text { CG: } 81.6 \\
(79.5 \text { to } 83.6) \\
p=0.76 \\
\text { Female gender, } \\
n \text { (\%): } \\
\text { Tx: } 47(60.3 \%) ; \\
\text { CG: } 47(67.1 \%) \text {, } \\
\text { p=0.38 } \\
\text { Race/Ethnicity: } \\
\text { NR } \\
\text { SES: NR }\end{array}$ & 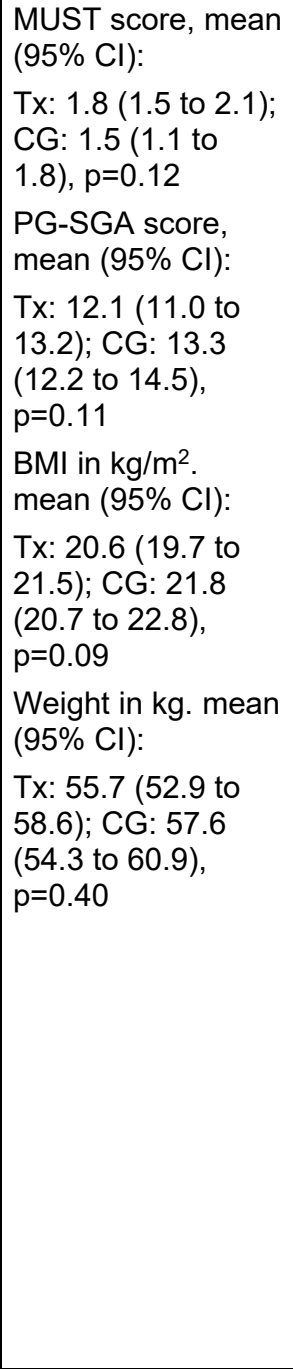 & 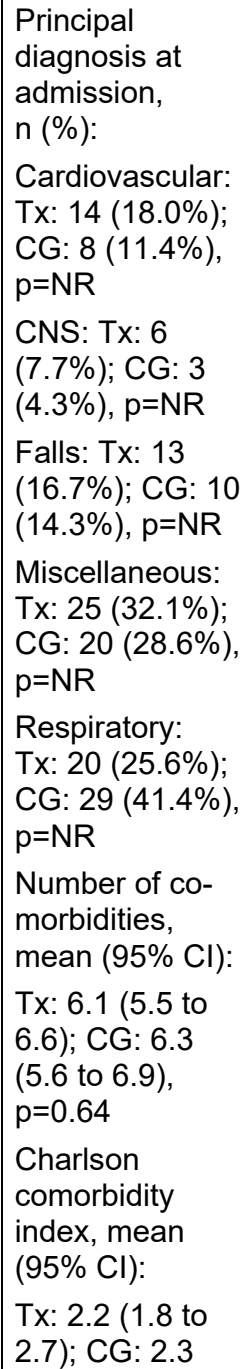 \\
\hline
\end{tabular}




\begin{tabular}{|c|c|c|c|c|c|c|c|}
\hline $\begin{array}{l}\text { Author/Year } \\
\text { Study Design } \\
\text { Countryl } \\
\text { Setting }\end{array}$ & Objective & $\begin{array}{l}\text { Patient Inclusion/ } \\
\text { Exclusion Criteria }\end{array}$ & $\begin{array}{l}\text { Intervention } \\
\text { Control }\end{array}$ & $\begin{array}{l}\text { Number of } \\
\text { Patients per } \\
\text { Study Arm } \\
\text { Follow-up } \\
\text { Duration }\end{array}$ & $\begin{array}{l}\text { Age } \\
\text { Gender } \\
\text { Race/Ethnicity } \\
\text { Socioeconomic } \\
\text { Status (SES) }\end{array}$ & $\begin{array}{l}\text { Screening } \\
\text { Instrument } \\
\text { Nutritional Status } \\
\text { BMI } \\
\text { Weight }\end{array}$ & $\begin{array}{l}\text { Underlying } \\
\text { Condition } \\
\text { Disease } \\
\text { Severity }\end{array}$ \\
\hline & & & & & & & $\begin{array}{l}(1.9 \text { to } 2.8), \\
p=0.82\end{array}$ \\
\hline $\begin{array}{l}\text { Starke et al. } \\
2011^{90} \\
\text { RCT } \\
\text { Switzerland }\end{array}$ & $\begin{array}{l}\text { Evaluate a routinely } \\
\text { manageable } \\
\text { concept for an } \\
\text { improved nutritional } \\
\text { care of } \\
\text { malnourished in- } \\
\text { hospital patients. }\end{array}$ & $\begin{array}{l}\text { Included adult patients } \\
\text { admitted to the } \\
\text { general medical ward } \\
\text { in a Swiss hospital } \\
\text { between January } 2007 \\
\text { and November } 2007 \\
\text { with an NRS-2002 } \\
\text { score } \geq 3 \text {. } \\
\text { Excluded patients with } \\
\text { no informed consent, } \\
\text { terminal condition, } \\
\text { expected stay <5 days } \\
\text { (judged by physician), } \\
\text { previous participation } \\
\text { in this study, patient } \\
\text { on starvation, on } \\
\text { parenteral nutrition, } \\
\text { and/or being on } \\
\text { dialysis. }\end{array}$ & $\begin{array}{l}\text { Intervention (Tx): } \\
\text { Individual nutritional } \\
\text { care, including a } \\
\text { detailed nutritional } \\
\text { assessment, } \\
\text { individual food } \\
\text { supply, fortification } \\
\text { of meals with } \\
\text { maltodextrin, } \\
\text { rapeseed oil, cream } \\
\text { and/or protein } \\
\text { powder, in-between } \\
\text { snacks and oral } \\
\text { nutritional } \\
\text { supplements. } \\
\text { Control (CG): } \\
\text { Standard nutritional } \\
\text { care, including the } \\
\text { prescription of oral } \\
\text { nutritional } \\
\text { supplements and } \\
\text { nutritional therapy } \\
\text { prescribed by the } \\
\text { physician } \\
\text { independently of this } \\
\text { study and according } \\
\text { to the routine ward } \\
\text { management. }\end{array}$ & $\begin{array}{l}\text { Tx: } 66 \\
\text { CG: } 66 \\
6 \text { months }\end{array}$ & $\begin{array}{l}\text { Age, mean (SD): } \\
\text { Tx: } 70 \text { (16); CG: } \\
75 \text { (11), p=0.091 } \\
\text { Gender: NR } \\
\text { Race/Ethnicity: } \\
\text { NR } \\
\text { SES: NR }\end{array}$ & $\begin{array}{l}\text { NRS-2002 score } \\
\text { for nutritional } \\
\text { status, mean (SD): } \\
\text { Tx: } 1.8(0.6) \text {; CG: } \\
1.7(0.7), p=0.233 \\
\text { NRS-2002 score } \\
\text { for severity of } \\
\text { disease, mean } \\
\text { (SD): } \\
\text { Tx: } 1.0(0.3) ; \text { CG: } \\
0.9(0.5), p=0.057 \\
\text { BMI in kg/m², } \\
\text { mean (SD): } \\
\text { Tx: } 24.6(5.3) ; C G: \\
24.1(4.9), p=0.527 \\
\text { Weight in kg, mean } \\
\text { (SD): } \\
\text { Tx: } 68.1(16.9) ; \\
\text { CG: } 66.1(16.2), \\
\text { p=0.504 }\end{array}$ & Diagnosis: NR \\
\hline
\end{tabular}

$\mathrm{AMI}=$ acute myocardial infarction; $\mathrm{BMI}=$ body mass index $\mathrm{CG}=$ control group CHD $=$ coronary heart disease; $\mathrm{CHF}=$ congestive heart failure; $\mathrm{CI}=$ confidence interval;

$\mathrm{CKD}=$ chronic kidney disease; $\mathrm{CNS}=$ central nervous system; COPD = chronic obstructive pulmonary disease; $\mathrm{CVA}=$ cerebrovascular accident; $\mathrm{CVD}=$ cardiovascular disease

$\mathrm{GI}=$ gastrointestinal; $\mathrm{HF}=$ heart failure; $\mathrm{HLD}=$ hyperlipidemia; $\mathrm{HTN}=$ hypertension; $\mathrm{Kg}=$ kilograms; $\mathrm{Kg} / \mathrm{m}^{2}=$ kilograms per meters squared; $\mathrm{LOS}=$ length of stay;

MNA = mini nutritional assessment; MUST = malnutrition universal screening tool; NR = not reported; NRS-2002 = nutrition risk screening 2002; NS = not significant;

$\mathrm{NT}=$ nutrition therapy; ONS = oral nutrition supplement; PEG = percutaneous endoscopic gastrostomy; PG-SGA = patient-generated subjective global assessment;

$\mathrm{PNA}=$ pulmonary nodular amyloidosis; $\mathrm{RCT}=$ randomized controlled trial; $\mathrm{SD}=$ standard deviation; $\mathrm{SE}=$ standard error; $\mathrm{SES}=$ socioeconomic status;

$\mathrm{SGA}=$ subjective global assessment; $\mathrm{Tx}=$ Treatment 


\section{Appendix E. Risk of Bias}

Table E-1. Risk of bias assessment for systematic reviews

\begin{tabular}{|c|c|c|c|c|c|c|c|}
\hline & Question & DiJkink 2020 & $\begin{array}{l}\text { Ney } \\
2019\end{array}$ & $\begin{array}{l}\text { Muscaritoli } \\
2017\end{array}$ & $\begin{array}{l}\text { Lew } \\
2016\end{array}$ & $\begin{array}{l}\text { Lin. } \\
2016\end{array}$ & $\begin{array}{l}\text { Gupta } \\
2011\end{array}$ \\
\hline \multirow[t]{6}{*}{$\begin{array}{l}\text { Study eligibility } \\
\text { criteria }\end{array}$} & $\begin{array}{l}\text { Did the review adhere to predefined objectives and eligibility } \\
\text { criteria? }\end{array}$ & Yes & Yes & Yes & Yes & Yes & Yes \\
\hline & Were the eligibility criteria appropriate for the review question? & Yes & Yes & Yes & Yes & Yes & $\begin{array}{l}\text { Prob } \\
\text { Yes }\end{array}$ \\
\hline & Were eligibility criteria unambiguous? & Yes & Yes & Yes & Yes & Yes & Yes \\
\hline & $\begin{array}{l}\text { Were all restrictions in eligibility criteria based on study } \\
\text { characteristics appropriate (e.g. date, sample size, study quality, } \\
\text { outcomes measured)? }\end{array}$ & Yes & Yes & Yes & Yes & Yes & Yes \\
\hline & $\begin{array}{l}\text { Were any restrictions in eligibility criteria based on sources of } \\
\text { information appropriate (e.g. publication status or format, language, } \\
\text { availability of data)? }\end{array}$ & Prob Yes & $\begin{array}{l}\text { Prob } \\
\text { Yes }\end{array}$ & Prob Yes & Yes & $\begin{array}{l}\text { Prob } \\
\text { Yes }\end{array}$ & $\begin{array}{l}\text { Prob } \\
\text { Yes }\end{array}$ \\
\hline & Overall Concern & Low & Low & Low & Low & Low & Low \\
\hline \multirow[t]{6}{*}{$\begin{array}{l}\text { Identification and } \\
\text { Selection of Studies }\end{array}$} & $\begin{array}{l}\text { Did the search include an appropriate range of } \\
\text { databases/electronic sources for published and unpublished } \\
\text { reports? }\end{array}$ & Yes & Yes & Yes & Yes & Yes & Yes \\
\hline & $\begin{array}{l}\text { Were methods additional to database searching used to identify } \\
\text { relevant reports? }\end{array}$ & Yes & Yes & Yes & Yes & Yes & Yes \\
\hline & $\begin{array}{l}\text { Were the terms and structure of the search strategy likely to } \\
\text { retrieve as many eligible studies as possible? }\end{array}$ & Prob Yes & Yes & Yes & Yes & Yes & Yes \\
\hline & $\begin{array}{l}\text { Were restrictions based on date, publication format, or language } \\
\text { appropriate? }\end{array}$ & Prob Yes & Yes & Yes & $\begin{array}{l}\text { Prob } \\
\text { Yes }\end{array}$ & $\begin{array}{l}\text { Prob } \\
\text { Yes }\end{array}$ & $\begin{array}{l}\text { Prob } \\
\text { Yes }\end{array}$ \\
\hline & Were efforts made to minimize error in selection of studies? & Prob Yes & Yes & Yes & Yes & Yes & Yes \\
\hline & Overall Concern & Low & Low & Low & Low & Low & Low \\
\hline \multirow{6}{*}{$\begin{array}{l}\text { Data Collection and } \\
\text { Study Appraisal }\end{array}$} & Were efforts made to minimize error in data collection? & Yes & Yes & Yes & Yes & Yes & Yes \\
\hline & $\begin{array}{l}\text { Were sufficient study characteristics available for both review } \\
\text { authors and readers to be able to interpret the results? }\end{array}$ & Prob Yes & Yes & Yes & Yes & Yes & Yes \\
\hline & Were all relevant study results collected for use in the synthesis? & Prob Yes & Yes & Yes & $\begin{array}{l}\text { Prob } \\
\text { Yes } \\
\end{array}$ & Yes & Yes \\
\hline & $\begin{array}{l}\text { Was risk of bias (or methodological quality) formally assessed } \\
\text { using appropriate criteria? }\end{array}$ & Prob Yes & Yes & Yes & Yes & Yes & No \\
\hline & Were efforts made to minimize error in risk of bias assessment? & Yes & Yes & Yes & Yes & Yes & No \\
\hline & Overall Concern & Low & Low & Low & Low & Low & High \\
\hline $\begin{array}{l}\text { Synthesis and } \\
\text { Findings }\end{array}$ & Did the synthesis include all studies that it should? & Prob Yes & $\begin{array}{l}\text { Prob } \\
\text { Yes }\end{array}$ & Prob Yes & $\begin{array}{l}\text { Prob } \\
\text { Yes }\end{array}$ & Yes & $\begin{array}{l}\text { Prob } \\
\text { Yes }\end{array}$ \\
\hline
\end{tabular}




\begin{tabular}{|c|c|c|c|c|c|c|c|}
\hline & Question & DiJkink 2020 & $\begin{array}{l}\text { Ney } \\
2019\end{array}$ & $\begin{array}{l}\text { Muscaritoli } \\
2017\end{array}$ & $\begin{array}{l}\text { Lew } \\
2016\end{array}$ & $\begin{array}{l}\text { Lin. } \\
2016\end{array}$ & $\begin{array}{l}\text { Gupta } \\
2011\end{array}$ \\
\hline & Were all pre-defined analyses reported or departures explained? & Prob Yes & \begin{tabular}{|l|} 
Prob \\
Yes
\end{tabular} & Prob Yes & \begin{tabular}{|l|} 
Prob \\
Yes
\end{tabular} & Yes & Yes \\
\hline & $\begin{array}{l}\text { Was the synthesis appropriate given the nature and similarity in the } \\
\text { research questions, study designs and outcomes across included } \\
\text { studies? }\end{array}$ & Yes & $\begin{array}{l}\text { Prob } \\
\text { Yes }\end{array}$ & Prob Yes & $\begin{array}{l}\text { Prob } \\
\text { Yes }\end{array}$ & Yes & $\begin{array}{l}\text { Prob } \\
\text { Yes }\end{array}$ \\
\hline & $\begin{array}{l}\text { Was between-study variation (heterogeneity) minimal or addressed } \\
\text { in the synthesis? }\end{array}$ & Yes & $\begin{array}{l}\text { Prob } \\
\text { Yes }\end{array}$ & Prob Yes & $\begin{array}{l}\text { Prob } \\
\text { Yes }\end{array}$ & Yes & $\begin{array}{l}\text { Prob } \\
\text { Yes }\end{array}$ \\
\hline & $\begin{array}{l}\text { Were the findings robust, e.g. as demonstrated through funnel plot } \\
\text { or sensitivity analyses? }\end{array}$ & Prob Yes & $\begin{array}{l}\text { Prob } \\
\text { Yes }\end{array}$ & Prob Yes & $\begin{array}{l}\text { Prob } \\
\text { Yes }\end{array}$ & Yes & $\begin{array}{l}\text { Prob } \\
\text { Yes }\end{array}$ \\
\hline & $\begin{array}{l}\text { Were biases in primary studies minimal or addressed in the } \\
\text { synthesis? }\end{array}$ & Prob Yes & $\begin{array}{l}\text { Prob } \\
\text { Yes }\end{array}$ & Prob Yes & $\begin{array}{l}\text { Prob } \\
\text { Yes }\end{array}$ & Yes & $\begin{array}{l}\text { Prob } \\
\text { Yes }\end{array}$ \\
\hline & Overall Concern & Low & Low & Low & Low & Low & Low \\
\hline \multicolumn{2}{|c|}{ Overall Risk of Bias Rating } & Low & Low & Low & Low & Low & Low* \\
\hline
\end{tabular}

*While the authors of this review did not formally assess the ROB of the included individual studies, they did report on critical information that allowed ECRI-Penn to infer the ROB of the studies. Only one of the 8 studies from this review addressing KQ1 controlled for important confounders. Thus, the ROB of most studies was rated "High". 
Table E-2. Risk of bias for randomized controlled trials (Cochrane ROB 2.0) for studies answering KQ 3

\begin{tabular}{|c|c|c|c|c|c|c|c|}
\hline Author & Outcomes & Randomization & $\begin{array}{l}\text { Effect of } \\
\text { Assignment }\end{array}$ & $\begin{array}{l}\text { Missing } \\
\text { Outcome } \\
\text { Data }\end{array}$ & $\begin{array}{l}\text { Measurement } \\
\text { of Outcome }\end{array}$ & $\begin{array}{l}\text { Selection } \\
\text { of Reported } \\
\text { Results }\end{array}$ & $\begin{array}{l}\text { Overall } \\
\text { RoB }\end{array}$ \\
\hline $\begin{array}{l}\text { Bonilla-Palomas } \\
2016\end{array}$ & Mortality, Readmission & Some Concerns & Low & Low & Low & \begin{tabular}{|l|} 
Some \\
Concerns \\
\end{tabular} & \begin{tabular}{|l} 
Some \\
Concerns \\
\end{tabular} \\
\hline Deutz 2016 & $\begin{array}{l}\text { Length of Stay, Mortality, Readmission, Activities of } \\
\text { Daily Living }\end{array}$ & Low & Low & $\begin{array}{l}\text { Some } \\
\text { Concerns }\end{array}$ & Low & Low & $\begin{array}{l}\text { Some } \\
\text { Concerns }\end{array}$ \\
\hline Gazzotti 2003 & $\begin{array}{l}\text { Discharge Disposition, Hospital Acquired } \\
\text { Conditions, Length of Stay, Mortality, Readmission }\end{array}$ & Low & Low & $\begin{array}{l}\text { Some } \\
\text { Concerns }\end{array}$ & Low & $\begin{array}{l}\text { Some } \\
\text { Concerns }\end{array}$ & $\begin{array}{l}\text { Some } \\
\text { Concerns }\end{array}$ \\
\hline Ha 2010 & Length of Stay, Mortality & Some Concerns & $\begin{array}{l}\text { Some } \\
\text { Concerns }\end{array}$ & $\begin{array}{l}\text { Some } \\
\text { Concerns }\end{array}$ & Low & Low & High \\
\hline Ha 2010 & Quality of Life* & Some Concerns & $\begin{array}{l}\text { Some } \\
\text { Concerns }\end{array}$ & High & $\begin{array}{l}\text { Some } \\
\text { Concerns }\end{array}$ & Low & High \\
\hline Holyday 2011 & Length of Stay, Mortality, Readmission & Some Concerns & Low & Low & Low & High & High \\
\hline Johansen 2004 & Hospital Acquired Conditions, Length of Stay & Low & Low & High & Low & $\begin{array}{l}\text { Some } \\
\text { Concerns }\end{array}$ & High \\
\hline Johansen 2004 & Quality of Life* & Low & Low & Low & $\begin{array}{l}\text { Some } \\
\text { Concerns }\end{array}$ & $\begin{array}{l}\text { Some } \\
\text { Concerns }\end{array}$ & High \\
\hline Munk 2014 & Length of Stay, Mortality & Some Concerns & Low & Low & Low & Low & $\begin{array}{l}\text { Some } \\
\text { Concerns }\end{array}$ \\
\hline Rufenacht 2011 & Mortality & Low & Low & Low & Low & $\begin{array}{l}\text { Some } \\
\text { Concerns } \\
\end{array}$ & $\begin{array}{l}\text { Some } \\
\text { Concerns }\end{array}$ \\
\hline Rufenacht 2011 & Quality of Life* & Low & High & Low & High & Low & High \\
\hline Schuetz 2019 & $\begin{array}{l}\text { Activities of Daily Living, Length of Stay, Mortality, } \\
\text { Readmissions }\end{array}$ & Low & Low & Low & Low & Low & Low \\
\hline Schuetz 2019 & Quality of Life* & Low & Low & Low & High & Low & High \\
\hline Sharma 2017 & $\begin{array}{l}\text { Discharge Disposition, Hospital Acquired } \\
\text { Conditions, Length of Stay }\end{array}$ & Low & $\begin{array}{l}\text { Some } \\
\text { Concerns }\end{array}$ & High & Low & Low & High \\
\hline Sharma 2017 & Quality of Life* & Low & High & \begin{tabular}{|l} 
Some \\
Concerns
\end{tabular} & High & Low & High \\
\hline Sharma 2017 & Readmissions $^{* *}$ & Low & High & High & Low & Low & High \\
\hline Starke 2011 & $\begin{array}{l}\text { Hospital Acquired Conditions, Length of Stay, } \\
\text { Mortality, Readmission }\end{array}$ & Some Concerns & Low & Low & Low & Low & $\begin{array}{l}\text { Some } \\
\text { Concerns }\end{array}$ \\
\hline Starke 2011 & Quality of Life* & Some Concerns & Low & High & Low & Low & High \\
\hline
\end{tabular}

Note: Colors in table are for visual effect and do not provide any additional information.

*Quality of life analyzed separately to account for domains influencing outcome assessment.

**For Sharma et al. 2017 patients received telehealth visits which could have potentially influenced the outcome of readmissions; thus, we rated the readmissions outcome separately from other objective outcomes. 


\section{Appendix F. Results From Included Studies}

Table F-1. Findings of reported outcomes of systematic reviews assessing the association of malnutrition with outcomes

\begin{tabular}{|c|c|c|c|c|c|c|c|c|c|}
\hline \begin{tabular}{|l} 
Author/Year \\
Type of \\
Patient \\
\end{tabular} & Mortality & Length of Stay & $\begin{array}{l}\text { 30-Day } \\
\text { Readmission }\end{array}$ & $\begin{array}{l}\text { Quality } \\
\text { of Life }\end{array}$ & $\begin{array}{l}\text { Functional } \\
\text { Status }\end{array}$ & $\begin{array}{l}\text { Activities } \\
\text { of Daily } \\
\text { Living }\end{array}$ & $\begin{array}{l}\text { Hospital } \\
\text { Acquired } \\
\text { Condition (HAC) }\end{array}$ & $\begin{array}{l}\text { Wound } \\
\text { Healing }\end{array}$ & $\begin{array}{l}\text { Discharge } \\
\text { Disposition }\end{array}$ \\
\hline $\begin{array}{l}\text { Dijkink et al. } \\
2020^{74} \\
\text { Trauma } \\
\text { Patients }\end{array}$ & 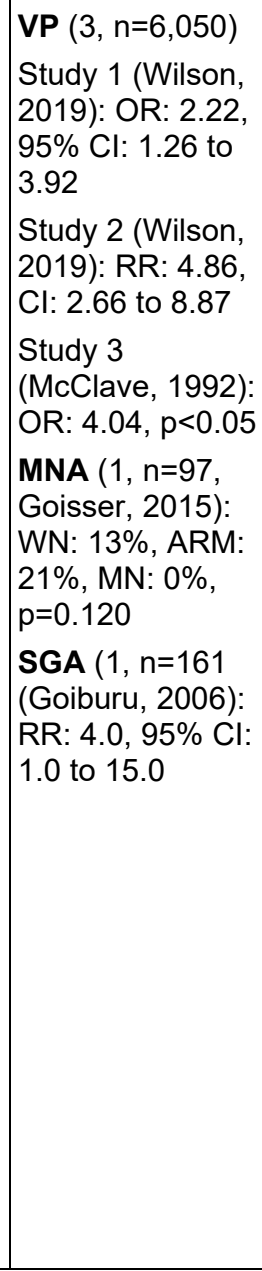 & $\begin{array}{l}\text { VP }(3, n=6,050) \\
\text { Study } 1 \text { (Wilson, } \\
2019): p=0.024 \\
\text { Study } 2 \text { (Wilson, } \\
2019): p<0.001 \\
\text { Study } 3 \text { (McClave, } \\
\text { 1992): OR: } 1.29 \text {, } \\
p<0.05 \\
\text { NRS (1, } n=521, \\
\text { Ihle, 2017): ND } \\
\text { MNA (2, } n=396) \\
\text { Study } 1 \text { (Goisser, } \\
\text { 2015): } p=0.388 \text {, } \\
\text { ND } \\
\text { Study 2 (Compan } \\
\text { et al. 1999): } \\
\text { positive } \\
\text { association } \\
\text { SGA (1, } n=161 \\
\text { (Goiburu, 2006): } \\
\text { RR: } 2.3,95 \% \text { Cl: } \\
1.2 \text { to 4.7 }\end{array}$ & $\begin{array}{l}\text { VP }(1, n=5,673, \\
\text { Wilson, } 2019): 2.0 \\
95 \% \text { Cl: } 1.55 \text { to } \\
2.57\end{array}$ & NR & NR & NR & $\begin{array}{l}\text { Sepsis } \\
\text { VP }(2, n=5673) \\
\text { Study 1 (Wilson, } \\
2019): 1.99, \\
95 \% \text { Cl: } 1.03 \text { to } \\
3.86 \\
\text { Study 2: (McClave, } \\
\text { 1992): OR: } 2.64, \\
\text { p<0.05 } \\
\text { Infection } \\
\text { VP (1, NR, } \\
\text { McClave, } 1992): \\
\text { OR: } 2.26, p<0.05 \\
\text { AE not described } \\
\text { VP }(2, n=6,050) \\
\text { Study } 1 \text { (Wilson, } \\
\text { 2019): positive } \\
\text { association } \\
\text { Study 2 (Wilson } \\
\text { 2019): RR: } 1.46, \\
\text { 95\% Cl: } 1.03 \text { to } \\
1.64 \\
\text { NRS (2, n=2,163) } \\
\text { Study } 1 \text { (Ille, } \\
\text { 2017): positive } \\
\text { association } \\
\text { (p<0.001) } \\
\text { Study 2 } \\
\text { (Wintermeyer } \\
\text { et al. 2019): } \\
\text { positive }\end{array}$ & NR & NR \\
\hline
\end{tabular}




\begin{tabular}{|c|c|c|c|c|c|c|c|c|c|}
\hline $\begin{array}{l}\text { Author/Year } \\
\text { Type of } \\
\text { Patient }\end{array}$ & Mortality & Length of Stay & $\begin{array}{l}\text { 30-Day } \\
\text { Readmission }\end{array}$ & $\begin{array}{l}\text { Quality } \\
\text { of Life }\end{array}$ & $\begin{array}{l}\text { Functional } \\
\text { Status }\end{array}$ & $\begin{array}{l}\text { Activities } \\
\text { of Daily } \\
\text { Living }\end{array}$ & $\begin{array}{l}\text { Hospital } \\
\text { Acquired } \\
\text { Condition (HAC) }\end{array}$ & $\begin{array}{l}\text { Wound } \\
\text { Healing }\end{array}$ & $\begin{array}{l}\text { Discharge } \\
\text { Disposition }\end{array}$ \\
\hline & & & & & & & $\begin{array}{l}\text { association } \\
(p<0.001) \\
\text { SGA }(1, n=161, \\
\text { Goiburu, } 2006) \text { : } \\
\text { RR: } 2.9,95 \% \mathrm{Cl} \text { : } \\
1.4 \text { to } 5.8\end{array}$ & & \\
\hline $\begin{array}{l}\text { Ney et al. } \\
2019^{75}\end{array}$ & 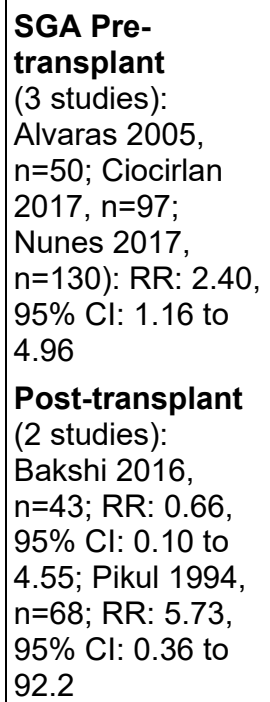 & $\begin{array}{l}\text { SGA (2 studies } \\
\text { Pikul, 1994, n=30; } \\
\text { Stephenson, 2001, } \\
\text { n=68): RR: } 4.35 \text {, } \\
95 \% \mathrm{Cl}: 5.42 \text { to } \\
22.69, \mathrm{I}^{2}=93 \%\end{array}$ & NR & NR & NR & NR & NR & NR & NR \\
\hline $\begin{array}{l}\text { Muscaritoli } \\
\text { et al. } 2017^{76} \\
\text { Various } \\
\text { Conditions/ } \\
\text { General } \\
\text { Medicine: } 7 \\
\text { COPD: } 3 \\
\text { Heart Failure: } 4 \\
\text { Pneumonia: } 1\end{array}$ & NR & NR & $\begin{array}{l}5 \text { prospective } \\
\text { cohort trials } \\
\text { showed positive } \\
\text { association b/w } \\
\text { MN and increase } \\
\text { in readmission. } \\
8 \text { studies showed } \\
\text { no independent } \\
\text { predictive } \\
\text { association of MN } \\
\text { for readmission. }\end{array}$ & NR & NR & NR & NR & NR & NR \\
\hline
\end{tabular}




\begin{tabular}{|c|c|c|c|c|c|c|c|c|c|}
\hline $\begin{array}{l}\text { Author/Year } \\
\text { Type of } \\
\text { Patient }\end{array}$ & Mortality & Length of Stay & \begin{tabular}{|l|} 
30-Day \\
Readmission
\end{tabular} & $\begin{array}{l}\text { Quality } \\
\text { of Life }\end{array}$ & $\begin{array}{l}\text { Functional } \\
\text { Status }\end{array}$ & \begin{tabular}{|l|} 
Activities \\
of Daily \\
Living
\end{tabular} & \begin{tabular}{|l|} 
Hospital \\
Acquired \\
Condition (HAC)
\end{tabular} & $\begin{array}{l}\text { Wound } \\
\text { Healing }\end{array}$ & $\begin{array}{l}\text { Discharge } \\
\text { Disposition }\end{array}$ \\
\hline & & & 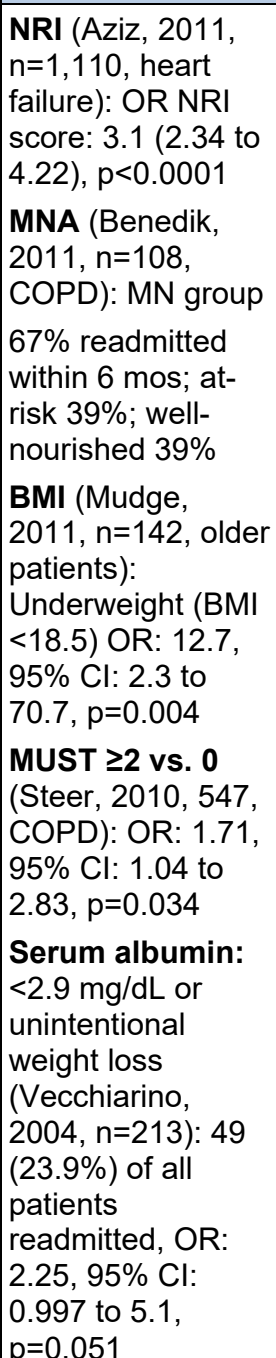 & & & & & & \\
\hline
\end{tabular}




\begin{tabular}{|c|c|c|c|c|c|c|c|c|c|}
\hline $\begin{array}{l}\text { Author/Year } \\
\text { Type of } \\
\text { Patient }\end{array}$ & Mortality & Length of Stay & $\begin{array}{l}\text { 30-Day } \\
\text { Readmission }\end{array}$ & $\begin{array}{l}\text { Quality } \\
\text { of Life }\end{array}$ & $\begin{array}{l}\text { Functional } \\
\text { Status }\end{array}$ & $\begin{array}{l}\text { Activities } \\
\text { of Daily } \\
\text { Living }\end{array}$ & $\begin{array}{l}\text { Hospital } \\
\text { Acquired } \\
\text { Condition (HAC) }\end{array}$ & $\begin{array}{l}\text { Wound } \\
\text { Healing }\end{array}$ & $\begin{array}{l}\text { Discharge } \\
\text { Disposition }\end{array}$ \\
\hline $\begin{array}{l}\text { Lew et al. } \\
2016^{77} \\
\text { Trauma/Acute } \\
\text { Care Patients }\end{array}$ & $\begin{array}{l}\text { SGA: } 2 \text { of } \\
5 \text { studies found } \\
\text { an association } \\
\text { b/w MN and } \\
\text { hosp. mortality: } \\
\text { Fontes, } 2014, \\
n=185, \text { OR: } 8.12 \text {, } \\
95 \% \text { Cl: } 2.94 \text { to } \\
22.42, p<0.05 ; \\
\text { Sheean, } 2013 \\
n=260, \text { WN: } 4.8, \\
\text { MN: } 23.0, \text { p<0.01 } \\
2 \text { studies non- } \\
\text { significant trend } \\
\text { (Capossi, } 2012, \\
\text { AOR: } 2.00, \\
95 \% \text { Cl: } 0.50 \text { to } \\
7.60 ; \text { Merli, } 2010, \\
\text { p=0.10) } \\
\text { MNA: } 2 \text { studies } \\
\text { Identified no } \\
\text { association } \\
\text { (Lomivorotov, } \\
2013, n=1193 ; \\
\text { Sheean, } 2013, \\
n=260) \\
\text { NRS-2002: } 1 \text { of } \\
2 \text { studies found } \\
\text { MN associated } \\
\text { with greater } \\
\text { hospital mortality } \\
\text { (Sheean, } 2013, \\
n=260 \text { adjusted } \\
\text { p=0.03) }\end{array}$ & $\begin{array}{l}\text { SGA: } 3 \text { of } \\
5 \text { studies found } \\
\text { MN was } \\
\text { associated with } \\
\text { longer Hosp and } \\
\text { ICU LOS: } \\
\text { Lomivorotov. 2013, } \\
\text { n=1193, } 2.00, \\
95 \% \text { Cl: } 1.10 \text { to } \\
3.70, p=0.02 ; \\
\text { Caporossi, } 2012, \\
\text { n=246, Hosp OR: } \\
2.80,95 \% \text { Cl: } 1.50 \\
\text { to 7.70, p<0.01; } \\
\text { Merli, 2010, n=38, } \\
\text { ICU HR: } 0.18, \\
\text { p<0.01, Hosp HR: } \\
0.20, \text { p<0.01 } \\
2 \text { studies reported } \\
\text { non-significant } \\
\text { trend (Sheean, } \\
2013 ; \text { Sheean, } \\
2010) \\
\text { MNA: } 2 \text { studies } \\
\text { identified no } \\
\text { association: } \\
\text { Lomivorotov, } 2013, \\
\text { n=1193; OR: } 1.40, \\
95 \% \text { Cl: } 0.70 \text { to } \\
2.30 ; \text { Sheean, } \\
2013, \text { n=260, adj } \\
\text { p=0.17 Hosp, } \\
\text { p=0.07 ICU }\end{array}$ & $\begin{array}{l}\text { SGA: } 1 \text { study } \\
\text { (Fontes, 2014, } \\
\mathrm{n}=185 \text { ) found } \mathrm{MN} \\
\text { was associated } \\
\text { with higher } \\
\text { incidence of ICU } \\
\text { readmission (OR: } \\
2.27,95 \% \mathrm{Cl}: 1.08 \\
\text { to } 4.80 ; \mathrm{p}<0.05)\end{array}$ & NR & NR & NR & $\begin{array}{l}\text { SGA: } 1 \text { study } \\
\text { (Merli, 2010, n=38) } \\
\text { found MN } \\
\text { independently } \\
\text { associated with } \\
\text { higher incidence of } \\
\text { infection (4.5 vs } \\
0.6 \text { episodes per } \\
\text { patient, adjusted, } \\
\text { p=0.0001) } \\
\text { MNA: } 1 \text { study } \\
\text { (Lomivorotov, } \\
2013, \text { n=1193) } \\
\text { found MN } \\
\text { independently } \\
\text { associated with } \\
\text { higher incidence of } \\
\text { postoperative } \\
\text { complications } \\
\text { (adjusted odds } \\
\text { ratio, } 1.60, \\
\text { 95\% Cl: } 1.10 \text { to } \\
2.20, \text { p<0.01) } \\
\text { NRS-2002: } \\
1 \text { study } \\
\text { (Lomivorotov, } \\
2013, \mathrm{n}=1193 \text { ) } \\
\text { identified no } \\
\text { association } \\
\text { between MN and } \\
\text { post-operative } \\
\text { complications }\end{array}$ & $\begin{array}{l}\text { SGA: 1 study } \\
\text { (Merli, 2010, } \\
n=38) \\
\text { identified no } \\
\text { association } \\
\text { between MN } \\
\text { and wound } \\
\text { healing or } \\
\text { graft } \\
\text { rejection }\end{array}$ & $\begin{array}{l}\text { SGA: } 1 \text { study } \\
\text { (Sheean, 2013, } \\
n=260) \text { found } \\
\text { MN was } \\
\text { associated with } \\
\text { a lower } \\
\text { percentage of } \\
\text { patients } \\
\text { discharged to } \\
\text { home (28.6\% } \\
\text { lower than their } \\
\text { well-nourished } \\
\text { counterparts; } \\
\text { adjusted } \\
\mathrm{p} \text { value=0.001) } \\
\text { NRS-2002: } \\
1 \text { study } \\
\text { (Sheean, 2013, } \\
n=260) \text { found } \\
\text { MN associated } \\
\text { with lower } \\
\text { discharge to } \\
\text { home (WN: } \\
74.9 \%, \text { MN: } \\
53.2 \%, p=0.01 \\
\text { MNA-SF: } \\
1 \text { study } \\
\text { (Sheean, 2013, } \\
n=260), \text { no } \\
\text { association btw } \\
\text { MN and } \\
\text { discharge } \\
\text { home, adj } \\
p=0.19 \\
\text { a }\end{array}$ \\
\hline
\end{tabular}




\begin{tabular}{|c|c|c|c|c|c|c|c|c|c|}
\hline $\begin{array}{l}\text { Author/Year } \\
\text { Type of } \\
\text { Patient }\end{array}$ & Mortality & Length of Stay & $\begin{array}{l}\text { 30-Day } \\
\text { Readmission }\end{array}$ & $\begin{array}{l}\text { Quality } \\
\text { of Life }\end{array}$ & $\begin{array}{l}\text { Functional } \\
\text { Status }\end{array}$ & $\begin{array}{l}\text { Activities } \\
\text { of Daily } \\
\text { Living }\end{array}$ & $\begin{array}{l}\text { Hospital } \\
\text { Acquired } \\
\text { Condition (HAC) }\end{array}$ & $\begin{array}{l}\text { Wound } \\
\text { Healing }\end{array}$ & $\begin{array}{l}\text { Discharge } \\
\text { Disposition }\end{array}$ \\
\hline & $\begin{array}{l}\text { MUST: Tripathy, } \\
2014, \mathrm{n}=109, \\
\text { found MN } \\
\text { associated with } \\
\text { 1-year mortality } \\
\text { (adjusted OR: } \\
2.94,95 \% \mathrm{Cl} \text { : } \\
1.10 \text { to } 8.00 \text { ) } \\
\text { MNA-SF: } 1 \text { study } \\
\text { (Sheean, } 2013, \\
\text { n=260) found MN } \\
\text { associated with } \\
\text { higher hospital } \\
\text { mortality (adj } \\
\text { p<0.01) }\end{array}$ & $\begin{array}{l}\text { NRS-2002: } 1 \text { of } \\
2 \text { studies found } \\
\text { malnutrition } \\
\text { associated with } \\
\text { increased LOS } \\
\text { (>2 days; OR: } \\
1.80,95 \% \text { Cl: } 1.10 \\
\text { to } 3.30 \\
\text { (Lomivorotov, } \\
2013, n-1193 \text { ) } \\
\text { MUST: } \\
\text { Lomivorotov, 2013, } \\
\text { n-1193 found no } \\
\text { association, AOR } \\
>2 \mathrm{~d} \text { of ICU stay: } \\
\text { 1.20, 95\% Cl: } 0.90 \\
\text { to } 2.00, p=0.33 \\
\text { MNA-SF: } 1 \text { study } \\
\text { (Sheean, } 2013, \\
n=260), \text { No } \\
\text { association btw } \\
\text { MN and LOS, adj } \\
p=0.06\end{array}$ & & & & & & & \\
\hline $\begin{array}{l}\text { Lin et al. } \\
2016^{78} \\
\text { Heart Failure }\end{array}$ & $\begin{array}{l}\text { MNA: } 4 \text { (Bonilla, } \\
\text { 2011, Sargento, } \\
\text { 2013, Aggarwal, } \\
\text { 2013, Suzuki, } \\
\text { 2015, 472): HR: } \\
4.32,95 \% \text { Cl: } \\
2.30 \text { to } 8.11) \\
\text { I }^{2}=0.0 \% \\
\text { MNA-SF: } 2 \text { (Yost, } \\
\text { 2014, n=162; } \\
\text { Sargento et al. } \\
2013, \text { n=50): HR: } \\
3.56,95 \% \text { Cl: } \\
\text { 1.41 to } 9.00\end{array}$ & $\begin{array}{l}\text { MNA (Yost, 2014, } \\
n=162): \text { ND } \\
\text { MNA-SF (Yost, } \\
2014, n=162): \text { ND } \\
\text { NRI (Aziz, 2011 } \\
n=1,110): \text { OR: } 1.7 \text {, } \\
95 \% \text { Cl: } 1.58 \text { to } \\
1.9 \\
\text { NRS (Tevik, } \\
n=131): \text { OR: } 2.99 \\
95 \% \text { Cl: } 1.33 \text { to } \\
6.73 \text {, confounded } \\
\text { with disease } \\
\text { severity }\end{array}$ & NR & NR & NR & NR & $\begin{array}{l}\text { NRS (Aziz, } 2011 \\
n=1,110): p<0.05 ; \\
\text { higher risk of } \\
\text { complication } \\
\text { associated with } \\
\text { MN }\end{array}$ & NR & NR \\
\hline
\end{tabular}




\begin{tabular}{|c|c|c|c|c|c|c|c|c|c|}
\hline $\begin{array}{l}\text { Author/Year } \\
\text { Type of } \\
\text { Patient }\end{array}$ & Mortality & Length of Stay & $\begin{array}{l}\text { 30-Day } \\
\text { Readmission }\end{array}$ & $\begin{array}{l}\text { Quality } \\
\text { of Life }\end{array}$ & $\begin{array}{l}\text { Functional } \\
\text { Status }\end{array}$ & $\begin{array}{l}\text { Activities } \\
\text { of Daily } \\
\text { Living }\end{array}$ & $\begin{array}{l}\text { Hospital } \\
\text { Acquired } \\
\text { Condition (HAC) }\end{array}$ & $\begin{array}{l}\text { Wound } \\
\text { Healing }\end{array}$ & $\begin{array}{l}\text { Discharge } \\
\text { Disposition }\end{array}$ \\
\hline & $\begin{array}{l}\text { NRI: } 3 \text { (Aziz, } \\
\text { 2011; Al-Naijer, } \\
\text { 2015; Gouya, } \\
\text { 2014, n=1,785): } \\
\text { HR: } 2.08, \\
\text { 95\% Cl: } 1.60 \text { to } \\
2.71 \\
\text { GNRI: } 3 \text { (Narumi, } \\
\text { 2013, Kinugasa } \\
\text { 2013, Kaneko, } \\
\text { 2015, n=978): } \\
\text { HR: } 3.11, \\
\text { 95\% Cl: } 1.69 \text { to } \\
5.74), I^{2}=70 \%\end{array}$ & & & & & & & & \\
\hline $\begin{array}{l}\text { Gupta et al. } \\
2011^{79} \\
\text { Cancer }\end{array}$ & NR & $\begin{array}{l}\text { LOS was } \\
\text { significantly higher } \\
\text { in the MN group } \\
\text { vs. WN group } \\
\text { SGA } \\
\text { Gastrointestinal } \\
\text { cancer (4): Wu, } \\
2010, n=505, \\
\text { mean WN: } \\
20.8 \text { days; mean } \\
\text { MN: } 29.1 \text { days, } \\
\text { p=0.001; Wu, } \\
2009, n=751, \\
\text { mean WN: } \\
17.1 \text { days, mean } \\
\text { MN: } 21.1 \text { days, } \\
\text { p=0.07; Wakahara, } \\
2007, n=262, W N: \\
\text { mean } 24 \text { days, } \\
\text { MN: mean } \\
57 \text { days, p=0.01; } \\
\text { Ulander, } 1998, \\
n=75,1994, W N: \\
<8 \text { days, MN: } \\
>14 \text { days }\end{array}$ & NR & NR & NR & NR & NR & NR & NR \\
\hline
\end{tabular}




\begin{tabular}{|c|c|c|c|c|c|c|c|c|c|}
\hline $\begin{array}{l}\text { Author/Year } \\
\text { Type of } \\
\text { Patient }\end{array}$ & Mortality & Length of Stay & $\begin{array}{l}\text { 30-Day } \\
\text { Readmission }\end{array}$ & $\begin{array}{l}\text { Quality } \\
\text { of Life }\end{array}$ & $\begin{array}{l}\text { Functional } \\
\text { Status }\end{array}$ & $\begin{array}{l}\text { Activities } \\
\text { of Daily } \\
\text { Living }\end{array}$ & $\begin{array}{l}\text { Hospital } \\
\text { Acquired } \\
\text { Condition (HAC) }\end{array}$ & $\begin{array}{l}\text { Wound } \\
\text { Healing }\end{array}$ & $\begin{array}{l}\text { Discharge } \\
\text { Disposition }\end{array}$ \\
\hline & & $\begin{array}{l}\text { Head and neck } \\
\text { cancer (1, } \\
\text { Shirodkar, 2005, } \\
n=266): \text { Median } \\
\text { postoperative } \\
\text { days=WN: } 5 \text { days, } \\
\text { MN: } 10 \text { days, } \\
\text { p<0.001 } \\
\text { Lymphoma (1, } \\
\text { Bauer, 2002, n=71 } \\
\text { retrospective): } \\
\text { WN: } 7.0 \text { days, MN: } \\
13.0 \text { days, p=0.02 } \\
\text { PG-SGA } \\
\text { Gynecologic } \\
\text { cancer (1, Laky, } \\
2010, n=157): \text { OR: } \\
6.89,2.48 \text { to } 19.2, \\
p<0.01 \\
\text { Multiple myeloma } \\
(1, n=66, \text { Horsley, } \\
2005, \\
\text { retrospective): } \\
\text { WN: } 16.9 \text { days, } \\
\text { MN: } 23 \text { days, } \\
p=0.002\end{array}$ & & & & & & & \\
\hline
\end{tabular}

$\mathrm{ARM}=$ at-risk of malnutrition; $\mathrm{CI}=$ confidence interval; GNRI = Geriatric Nutritional Risk Index; LOS = length of stay; MN = malnourished; MNA = mini nutritional assessment; MNA-SF = MNA-short form; MUST = malnutrition universal screening tool; NO indicates no association identified or no important difference found between malnourished and well-nourished; NR = not reported; NRI = Nutritional Risk Index; NRS-2002 = nutrition risk screening 2002; PG-SGA = patient-generated subjective global assessment; RCT = randomized controlled trial; $\mathrm{SD}=$ standard deviation; $\mathrm{SE}=$ standard error; $\mathrm{SES}=$ socioeconomic status; $\mathrm{SGA}=$ subjective global assessment; $\mathrm{VP}=$ visceral proteins; $\mathrm{WN}=$ well-nourished 
Table F-2. Findings of reported outcomes on effectiveness of malnutrition interventions

\begin{tabular}{|c|c|c|c|c|c|c|c|c|}
\hline Author/Year & Mortality & Length of Stay & Readmissions & Quality of Life & $\begin{array}{l}\text { Activities of } \\
\text { Daily Living }\end{array}$ & $\begin{array}{l}\text { Hospital Acquired } \\
\text { Condition (HAC) }\end{array}$ & \begin{tabular}{|l} 
Discharge \\
Disposition
\end{tabular} & Adverse Events \\
\hline $\begin{array}{l}\text { Bonilla- } \\
\text { Palomas } \\
\text { et al. } 2016^{80}\end{array}$ & $\begin{array}{l}\text { 1-month mortality, } \\
\text { \%: } \\
\text { Tx: 0.07; CG: } 0.11, \\
\text { p=NR } \\
\text { 3-month death from } \\
\text { any cause, \%: } \\
\text { Tx: 0.14; CG: } 0.26, \\
\text { p=NR } \\
\text { 6-month death from } \\
\text { any cause, \%: } \\
\text { Tx: 0.15; CG: } 0.36, \\
\text { p=NR } \\
\text { 12-month death } \\
\text { from any cause, \%: } \\
\text { Tx: } 0.203 ; \text { CG: } \\
0.475, p=0.003\end{array}$ & NR & $\begin{array}{l}\text { 1-month } \\
\text { readmission, \%: } \\
\text { Tx: 0.00; CG: } \\
\text { 0.09, p=NR } \\
\text { 3-month } \\
\text { readmission, \%: } \\
\text { Tx: 0.04; CG: } \\
\text { 0.22, p=NR } \\
\text { 6-month } \\
\text { readmission, \%: } \\
\text { Tx: 0.08; CG: } \\
\text { 0.35, p=NR } \\
\text { *12-month } \\
\text { readmission, \%: } \\
\text { Tx: 0.114; CG: } \\
\text { 0.433, p=0.001 }\end{array}$ & NR & NR & NR & NR & NR \\
\hline $\begin{array}{l}\text { Deutz et al. } \\
2016^{81}\end{array}$ & $\begin{array}{l}\text { 30-day mortality, } \%: \\
\text { ONS: } 2.9 \% \text {; CG: } \\
6.2 \%, p=0.049 \\
60 \text {-day mortality, } \%: \\
\text { ONS: } 4.2 \% \text {; CG: } \\
\text { 8.7\%, p=0.020 } \\
\text { 90-day mortality, } \%: \\
\text { ONS: } 4.8 \% \text {; CG: } \\
9.7 \%, p=0.018 \\
\text { The NNT to prevent } \\
1 \text { death, } 95 \% \text { Cl: } \\
20.3(10.9 \text { to } 121.4)\end{array}$ & $\begin{array}{l}\text { LOS in days, mean } \\
\text { (SD): } \\
\text { ONS: } 5.0(3.2) ; \\
\text { CG: } 5.1(3.6) \\
\text { p=NS }\end{array}$ & $\begin{array}{l}\text { 30-day } \\
\text { readmission, \%: } \\
\text { ONS: } 15.0 \% \text {; } \\
\text { CG: } 13.9 \% \text {, } \\
\text { p=0.697 } \\
\text { 60-day } \\
\text { readmission, \%: } \\
\text { ONS: } 20.4 \% \text {; } \\
\text { CG: } 21.4 \%, \\
\text { p=0.780 } \\
\text { 90-day } \\
\text { readmission, \%: } \\
\text { ONS: } 25.2 \% \text {; } \\
\text { CG: } 25.6 \%, \\
\text { p=NS }\end{array}$ & NR & $\begin{array}{l}\text { Katz score, } \\
\text { median }(\mathrm{Q} 1, \\
\text { Q3): } \\
\text { Tx: } 6(6,6) ; \\
\text { CG: } 6(6,6) \text {, } \\
\text { p=NR }\end{array}$ & NR & NR & $\begin{array}{l}\text { Adverse events: } \\
\text { ONS, n (\%): } \\
136(44) ; \text { CG: } \\
146(47), p=N S \\
\text { Serious adverse } \\
\text { events, n (\%): } \\
\text { ONS: } 92(30) ; \\
\text { CG: } 82(26), \\
p=N S\end{array}$ \\
\hline
\end{tabular}




\begin{tabular}{|c|c|c|c|c|c|c|c|c|}
\hline Author/Year & Mortality & Length of Stay & Readmissions & Quality of Life & $\begin{array}{l}\text { Activities of } \\
\text { Daily Living }\end{array}$ & $\begin{array}{l}\text { Hospital Acquired } \\
\text { Condition (HAC) }\end{array}$ & \begin{tabular}{|l} 
Discharge \\
Disposition
\end{tabular} & Adverse Events \\
\hline $\begin{array}{l}\text { Gazzotti } \\
\text { et al. } 2003^{82}\end{array}$ & $\begin{array}{l}\text { All-cause mortality, } \\
n: \\
\text { ONS: } 2 \text {; CG: } 2 \text {, } \\
p=N R\end{array}$ & $\begin{array}{l}\text { LOS in days, mean } \\
\text { (SD): } \\
\text { ONS: } 21.2(10.1) ; \\
\text { CG: } 19.8(15.1) \\
p=0.19\end{array}$ & $\begin{array}{l}\text { 60-day } \\
\text { readmission, n: } \\
\text { ONS: 4; CG: } 3 \text {, } \\
\text { p=NR }\end{array}$ & NR & NR & NR & $\begin{array}{l}\text { Discharged } \\
\text { Home, \%: } \\
\text { ONS: } 66.7 \% \text {; } \\
\text { CG: } 65 \% \\
\text { p=NR }\end{array}$ & $\begin{array}{l}\text { Minor side } \\
\text { effects, } n: \\
\text { ONS: 5; CG: } 0 \text {, } \\
p=N R\end{array}$ \\
\hline $\begin{array}{l}\text { Ha et al. } \\
2010^{83}\end{array}$ & $\begin{array}{l}\text { All-cause mortality, } \\
n: \\
\text { Tx: } 12 ; C G: 10 \\
p=N R\end{array}$ & $\begin{array}{l}\text { LOS in days, } \\
\text { median (Range): } \\
\text { Tx: } 12 \text { ( } 2 \text { to } 54) ; \\
\text { CG: } 13 \text { ( } 3 \text { to } 55) \text {, } \\
\text { p=NS }\end{array}$ & NR & $\begin{array}{l}\text { Increase in } \\
\text { EQ-5D VAS } \\
\text { score, Median } \\
\text { (Range): } \\
\text { Tx: } 10 \text { (-80 to } \\
60) ; \text { CG: } 0 \text { (-35 } \\
\text { to 70), p=0.009 } \\
\text { Patients with } \\
\text { an increase in } \\
\text { EQ-5D Score: } \\
\text { Tx: 71.4\%; CG: } \\
37.2 \%, p=0.003\end{array}$ & NR & NR & NR & NR \\
\hline $\begin{array}{l}\text { Holyday } \\
\text { et al. } 2011^{84}\end{array}$ & $\begin{array}{l}\frac{\text { At Risk }}{\text { All-cause mortality, }} \\
\mathrm{n}: \\
\text { Tx: 1; CG: } 0, p=N R \\
\text { Malnourished } \\
\text { All-cause mortality, } \\
\mathrm{n}: \\
\text { Tx: } 2 ; \mathrm{CG}: 1, \mathrm{p}=\mathrm{NR}\end{array}$ & $\begin{array}{l}\frac{A t \text { Risk }}{\text { LOS, excluding }} \\
\text { deaths, mean } \\
\text { (SE): } \\
\text { Tx: } 13.8 \text { (1.6); CG: } \\
11.0 \text { (1.4), p=0.20 } \\
\text { LOS, adjusted for } \\
\text { deaths, mean } \\
\text { (SE): } \\
\text { Tx: } 14.5 \text { (1.8); CG: } \\
11.0 \text { (1.4), p-0.097 } \\
\text { Malnourished } \\
\text { LOS, excluding } \\
\text { deaths, mean } \\
\text { (SE): } \\
\text { Tx: } 10.6(1.6) ; \text { CG: } \\
19.5 \text { (3.0), p=0.013 }\end{array}$ & $\begin{array}{l}\text { At Risk } \\
\text { 1-month } \\
\text { readmission, } \\
\text { mean (SE): } \\
\text { Tx: } 0.11(0.05) ; \\
\text { CG: } 0.10(0.05), \\
p=0.90 \\
\text { 6-month } \\
\text { readmission, } \\
\text { mean (SE): } \\
\text { Tx: } 0.37(0.09) ; \\
\text { CG: } 0.62(0.12), \\
p=0.11 \\
\text { Malnourished } \\
\text { 1-month } \\
\text { readmission, } \\
\text { mean (SE): } \\
\text { Tx: } 0.00(0.00) ; \\
\text { CG: } 0.16(0.09), \\
p=0.083\end{array}$ & NR & NR & NR & NR & NR \\
\hline
\end{tabular}




\begin{tabular}{|c|c|c|c|c|c|c|c|c|}
\hline Author/Year & Mortality & Length of Stay & Readmissions & Quality of Life & $\begin{array}{l}\text { Activities of } \\
\text { Daily Living }\end{array}$ & $\begin{array}{l}\text { Hospital Acquired } \\
\text { Condition (HAC) }\end{array}$ & $\begin{array}{l}\text { Discharge } \\
\text { Disposition }\end{array}$ & Adverse Events \\
\hline & & \begin{tabular}{|l|} 
LOS, adjusted for \\
deaths, mean \\
(SE): \\
Tx: $11.2(1.5)$; CG: \\
$19.8(3.0), p=0.054$
\end{tabular} & $\begin{array}{l}\text { 6-month } \\
\text { readmission, } \\
\text { mean }(\mathrm{SE}) \text { : } \\
\text { Tx: } 0.50(0.31) ; \\
\text { CG: } 0.63(0.14) \text {, } \\
\text { p=0.31 }\end{array}$ & & & & & \\
\hline $\begin{array}{l}\text { Johansen } \\
\text { et al. } 2004^{85}\end{array}$ & $\begin{array}{l}\text { All-cause mortality, } \\
\text { n: } \\
\text { Tx: 9; CG: 6, p=NR }\end{array}$ & $\begin{array}{l}\text { LOS until } 28 \text { days, } \\
\text { mean (SD): } \\
\text { Tx: } 11.2(0.7) \text {; CG: } \\
12.2(0.7), p=N S\end{array}$ & NR & $\begin{array}{l}\text { Change in } \\
\text { SF-36 quality of } \\
\text { life MCS, mean } \\
\text { (SE): } \\
\text { Tx: } 2.2(2.5) ; \\
\text { CG: } 3.3(2.0) \text {, } \\
\text { p=NR } \\
\text { Change in } \\
\text { SF-36 quality of } \\
\text { life PCS, mean } \\
\text { (SE): } \\
\text { Tx: } 2.4(1.3) ; \\
\text { CG: } 0.2(1.5) \text {, } \\
\text { p=NR }\end{array}$ & NR & $\begin{array}{l}\text { Incidence of minor } \\
\text { complications, } \mathrm{n}: \\
\text { Tx: 18; CG: } 14, \\
\mathrm{p}=\mathrm{NR} \\
\text { Incidence of major } \\
\text { complications, } \mathrm{n}: \\
\text { Tx: } 5 ; \mathrm{CG}: 0, \mathrm{p}=\mathrm{NR}\end{array}$ & NR & NR \\
\hline $\begin{array}{l}\text { Munk et al. } \\
2014^{86}\end{array}$ & $\begin{array}{l}\text { All-cause mortality, } \\
\text { n: } \\
\text { Tx: } 1 ; C G: 1, p=N R\end{array}$ & $\begin{array}{l}\text { LOS from } \\
\text { admission to } \\
\text { discharge, mean } \\
\text { (SD): } \\
\text { Tx: } 15 \text { (10); CG: } \\
14(8), p=0.38 \\
\text { LOS, inclusion to } \\
\text { discharge, mean } \\
\text { (SD): } \\
\text { Tx: } 10 \text { (8); CG: } 10 \\
\text { (8), p=0.73 }\end{array}$ & NR & NR & NR & NR & NR & NR \\
\hline
\end{tabular}




\begin{tabular}{|c|c|c|c|c|c|c|c|c|}
\hline Author/Year & Mortality & Length of Stay & Readmissions & Quality of Life & $\begin{array}{l}\text { Activities of } \\
\text { Daily Living }\end{array}$ & $\begin{array}{l}\text { Hospital Acquired } \\
\text { Condition (HAC) }\end{array}$ & \begin{tabular}{|l} 
Discharge \\
Disposition
\end{tabular} & Adverse Events \\
\hline \begin{tabular}{|l|} 
Rufenacht \\
et al. 201187
\end{tabular} & $\begin{array}{l}\text { All-cause mortality, } \\
n: \\
\text { Tx: 4; ONS: } 1 \\
p=N R\end{array}$ & NR & NR & $\begin{array}{l}\text { 30-day change } \\
\text { in EQ-5D VAS } \\
\text { score, mean } \\
\text { (SD): } \\
\text { Tx: } 28.5 \text { (20.6); } \\
\text { ONS: } 17.1 \\
\text { (20.4), p=NR } \\
\text { 30-day change } \\
\text { in FAACT total } \\
\text { score, mean: } \\
\text { Tx: 9.7; CG: } \\
\text { 10.7, p=NR } \\
\text { 60-day change } \\
\text { in FAACT total } \\
\text { score, mean: } \\
\text { Tx: 29.9; CG: } \\
\text { 24.0, p=NR }\end{array}$ & NR & NR & NR & NR \\
\hline \begin{tabular}{|l|} 
Schuetz \\
et al. $2019^{88}$
\end{tabular} & $\begin{array}{l}\text { All-cause mortality, } \\
\text { n (\%): } \\
\text { Tx: } 73(7 \%) ; \text { CG: } \\
100(10 \%), p=0.011\end{array}$ & $\begin{array}{l}\text { LOS in days, mean } \\
\text { (SD): } \\
\text { Tx: } 9.5(7.0) ; \text { CG: } \\
9.6(6.1), p=0.46\end{array}$ & \begin{tabular}{|l|} 
30-day \\
readmission, \\
n (\%): \\
Tx: 89 (9\%); CG: \\
$91(9 \%), p=0.96$
\end{tabular} & $\begin{array}{l}\text { EQ-5D index, } \\
\text { mean (SD): } \\
\text { Tx: } 0.75(0.32) ; \\
\text { CG: } 0.73 \\
(0.34), p=0.018 \\
\text { EQ-5D VAS, } \\
\text { mean (SD): } \\
\text { Tx: } 59(26) ; \\
\text { CG: } 56(29), \\
\text { p<0.0001 }\end{array}$ & $\begin{array}{l}\text { Barthel } \\
\text { index, mean } \\
\text { (SD): } \\
\text { Tx: } 88(26) ; \\
\text { CG: } 85(30) \text {, } \\
\text { p=0.006 } \\
\text { Decline in } \\
\text { functional } \\
\text { status of } \\
\geq 10 \%, n(\%) \text { : } \\
\text { Tx: } 35(4 \%) ; \\
\text { CG: } 55(6 \%) \text {, } \\
\text { p=0.034 }\end{array}$ & $\begin{array}{l}\text { Major complications, } \\
\text { n (\%): } \\
\text { Any major } \\
\text { complications: } \\
\text { Tx: } 74 \text { (7\%); CG: } 76 \\
(8 \%), p=0.79 \\
\text { Acute kidney failure: } \\
\text { Tx: } 32 \text { (3\%); CG: } 31 \\
(3 \%), p=0.96 \\
\text { GI disease: } \\
\text { Tx: } 9(1 \%) \text {; CG: } 15 \\
(1 \%), p=0.19 \\
\text { Major cardiovascular } \\
\text { event: } \\
\text { Tx: } 8(1 \%) \text {; CG: } 7 \\
(1 \%), p=0.84 \\
\text { Nosocomial infection: } \\
\text { Tx: } 40 \text { (4\%); CG: } 39 \\
(4 \%), p=0.98\end{array}$ & NR & $\begin{array}{l}\text { All side effects, } \mathrm{n} \\
\text { (\%): } \\
\text { Tx: } 162(16 \%) ; \\
\text { CG: } 145(14 \%), \\
\text { p=0.26 } \\
\text { Complications } \\
\text { due to enteral } \\
\text { feeding or } \\
\text { parental nutrition: } \\
\text { Tx: } 5(<1 \%) \text {; CG: } \\
3(<1 \%), p=0.51 \\
\text { GI side effects: } \\
\text { Tx: } 43(4 \%) ; \text { CG: } \\
40 \text { (4\%), p=0.66 } \\
\text { Liver or gall } \\
\text { bladder } \\
\text { dysfunction: } \\
\text { Tx: } 4(<1 \%) ; \text { CG: } \\
7 \text { (1\%), p=0.34 }\end{array}$ \\
\hline
\end{tabular}




\begin{tabular}{|c|c|c|c|c|c|c|c|c|}
\hline Author/Year & Mortality & Length of Stay & Readmissions & Quality of Life & $\begin{array}{l}\text { Activities of } \\
\text { Daily Living }\end{array}$ & $\begin{array}{l}\text { Hospital Acquired } \\
\text { Condition (HAC) }\end{array}$ & \begin{tabular}{|l|} 
Discharge \\
Disposition
\end{tabular} & Adverse Events \\
\hline & & & & & & $\begin{array}{l}\text { Respiratory failure: } \\
\text { Tx: } 14(1 \%) ; C G: 13 \\
(1 \%), p=0.89\end{array}$ & & $\begin{array}{l}\text { Refeeding } \\
\text { syndrome: } \\
\text { Tx: } 86(8 \%) \text {; CG: } \\
73(7 \%), p=0.27 \\
\text { Severe } \\
\text { hyperglycemia: } \\
\text { Tx: } 48(5 \%) \text {; CG: } \\
46(5 \%), p=0.80\end{array}$ \\
\hline $\begin{array}{l}\text { Sharma et al. } \\
2017^{89}\end{array}$ & \begin{tabular}{|l|} 
All-cause mortality, \\
n (\%): \\
Tx: $23(29.5 \%) ;$ CG: \\
$22(31.0 \%), p=0.84$ \\
In-hospital mortality, \\
n (\%): \\
Tx: $7(9.0 \%) ;$ CG: 1 \\
$(1.4 \%), p=0.09$ \\
3 months post- \\
discharge, n: \\
Tx: $14 ;$; CG: 12, \\
p=NR
\end{tabular} & $\begin{array}{l}\text { Acute LOS in } \\
\text { days, median } \\
\text { (IQR): } \\
\text { Tx: } 5.0(3.0 \text { to } 8.4) \text {; } \\
\text { CG: } 8.8(4.1 \text { to } \\
13.9), p=0.007 \\
\text { Total LOS, } \\
\text { inclusive of } \\
\text { hospital at home } \\
\text { time, median } \\
\text { (IQR): } \\
\text { Tx: } 5.4(3.1 \text { to } \\
11.2) ; \text { CG: } 11.4 \\
\text { (5 to } 21.6), p=0.01\end{array}$ & $\begin{array}{l}\text { Total } \\
\text { readmissions, } \\
\text { n (\%): } \\
\text { Tx: } 46 \text { (59.0\%); } \\
\text { CG: } 46(64.8 \%) \text {, } \\
\text { p=0.47 } \\
\text { 1-month } \\
\text { readmissions, } \\
\text { n (\%): } \\
\text { Tx: } 14(18.0 \%) ; \\
\text { CG: } 17(23.9 \%) \text {, } \\
\text { p=0.37 } \\
\text { 3-month } \\
\text { readmissions, } \\
\text { n (\%): } \\
\text { Tx: } 26(33.3 \%) ; \\
\text { CG: } 29(40.9 \%), \\
\text { p=0.34 } \\
\text { 6-month } \\
\text { readmissions, } \\
\text { n (\%): } \\
\text { Tx: } 37 \text { (47.4\%); } \\
\text { CG: } 35(50.0 \%), \\
\text { p=0.82 }\end{array}$ & $\begin{array}{l}\text { EQ-5D } 5 \text { level } \\
\text { index, mean } \\
\text { (95\% Cl): } \\
\text { Tx: } 0.770 \\
(0.721 \text { to } \\
0.818) ; \text { CG: } \\
0.740 \text { (0.674 to } \\
0.805), p=0.45 \\
\text { EQ-5D VAS } \\
\text { score, mean } \\
\text { (95\% Cl): } \\
\text { Tx: } 61.2 \text { (56.8 } \\
\text { to } 65.6) ; C G: \\
52.4(45.2 \text { to } \\
59.7), p=0.03\end{array}$ & NR & $\begin{array}{l}\text { Proportion of patients } \\
\text { with complications, } \\
\text { n (\%): } \\
\text { Tx: } 21(26.9 \%) ; \text { CG: } \\
23(32.4 \%), p=0.47 \\
\text { Total complications, } \\
\text { mean }(95 \% \text { Cl): } \\
\text { Tx: } 0.65(0.33 \text { to } \\
0.98) ; \text { CG: } 0.73(0.41 \\
\text { to } 1.05), p=0.73 \\
\text { Infective } \\
\text { complications, } n(\%): \\
\text { Tx: } 9(11.5 \%) ; \text { CG: } 7 \\
(9.9 \%), p=0.74 \\
\text { Non-infective } \\
\text { complications, } n(\%): \\
\text { Tx: } 19(24.4 \%) ; \text { CG: } \\
21(29.6 \%), p=0.48\end{array}$ & $\begin{array}{l}\text { Proportion of } \\
\text { patients } \\
\text { discharged to } \\
\text { residential } \\
\text { facility, n (\%): } \\
\text { Tx: } 6(7.7 \%) ; \\
\text { CG: } 6 \\
(8.5 \%) \\
\text { p }=0.09\end{array}$ & NR \\
\hline
\end{tabular}




\begin{tabular}{|c|c|c|c|c|c|c|c|c|}
\hline Author/Year & Mortality & Length of Stay & Readmissions & Quality of Life & $\begin{array}{l}\text { Activities of } \\
\text { Daily Living }\end{array}$ & $\begin{array}{l}\text { Hospital Acquired } \\
\text { Condition (HAC) }\end{array}$ & $\begin{array}{l}\text { Discharge } \\
\text { Disposition }\end{array}$ & Adverse Events \\
\hline $\begin{array}{l}\text { Starke et al. } \\
2011^{90}\end{array}$ & $\begin{array}{l}\text { During-study } \\
\text { mortality, n: } \\
\text { Tx: 2; CG: } 5 \text {, } \\
\text { p=0.440 } \\
\text { Mortality during } \\
\text { follow-up period, n: } \\
\text { Tx: } 9 \text {; CG: } 6, \\
p=0.585\end{array}$ & $\begin{array}{l}\text { Admission to } \\
\text { actual discharge, } \\
\text { mean (SD): } \\
\text { Tx: } 15.7(9.2) ; C G: \\
15.9(10.7), \\
\text { p=0.843 } \\
\text { Admission to } \\
\text { possible } \\
\text { discharge, mean } \\
\text { (SD): } \\
\text { Tx: } 13.8(7.1) ; \text { CG: } \\
14.9(10.2), \\
\text { p=0.458 } \\
\text { Hospital LOS, } \\
\text { mean (SD): } \\
\text { Tx: } 17.0(10.4) ; \\
\text { CG: } 18.6(17.1), \\
\text { p=0.913 }\end{array}$ & $\begin{array}{l}\text { 6-month } \\
\text { readmissions, } \\
\text { n (\%): } \\
\text { IG: } 17(27 \%) \\
\text { CG: } 28(46 \%) \\
p=0.027\end{array}$ & $\begin{array}{l}\text { SF-36 MCS, } \\
\text { mean (SD): } \\
\text { Tx: } 50 \text { (11); } \\
\text { CG: } 51(11), \\
\text { p=0.640 } \\
\text { SF-36 PCS, } \\
\text { mean (SD): } \\
\text { Tx: } 37(11) ; \\
\text { CG: } 32(9) \text {, } \\
\text { p=0.033 }\end{array}$ & NR & 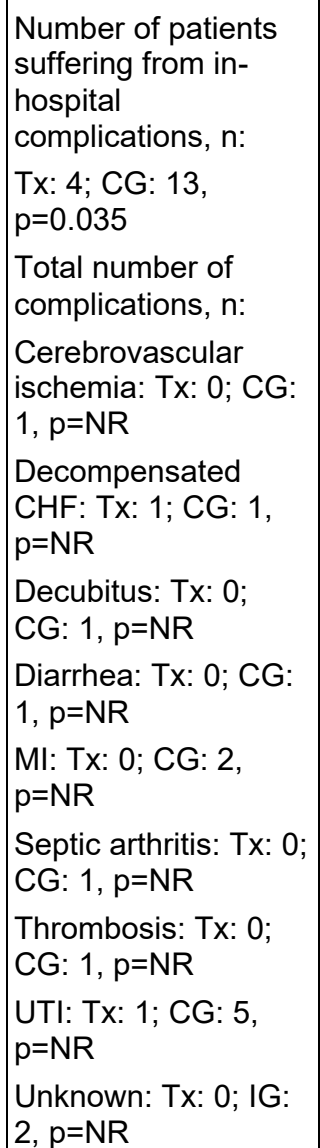 & NR & NR \\
\hline
\end{tabular}

$\mathrm{CG}=$ control group; $\mathrm{CHF}=$ congestive heart failure; $\mathrm{CI}=$ confidence interval; EQ-5D = EuroQol 5 dimension; FAACT = Functional Assessment of Anorexia/Cachexia Therapy; $\mathrm{GI}=$ gastrointestinal $; \mathrm{HF}=$ heart failure; $\mathrm{HR}=$ hazard ratio; $\mathrm{IQR}=$ interquartile range; $\mathrm{LOS}=$ length of stay; $\mathrm{MCS}=$ mental component summary; $\mathrm{MI}=$ myocardial infarction; $\mathrm{NNT}=$ number needed to treat; NR = not reported; NS = not significant; ONS = oral nutrition supplement; PCS = physical component summary; $\mathrm{Q} 1=$ first quartile; $\mathrm{Q} 3=$ third quartile; $\mathrm{QoL}=$ quality of life; $\mathrm{RR}=$ relative risk; $\mathrm{SD}=$ standard deviation; $\mathrm{SF}-36=36$ item short form health survey; Tx = treatment; VAS = visual analog scale *1-month, 3-month, and 6-month data extracted from survival analysis curves using a web-based numerical data extraction tool (WebPlotDigitizer v 4.4) to estimate graphical data. 12-month mortality data matched text data, although, the survival analysis plot portrayed different results than described in text (Treatment 10.2\% vs Control Group 36.1\%). Data reported are from the survival analysis. 


\section{Appendix G. Forest Plots of Additional Analysis}

Figure G-1. Effect of hospital-initiated interventions on mortality, all studies

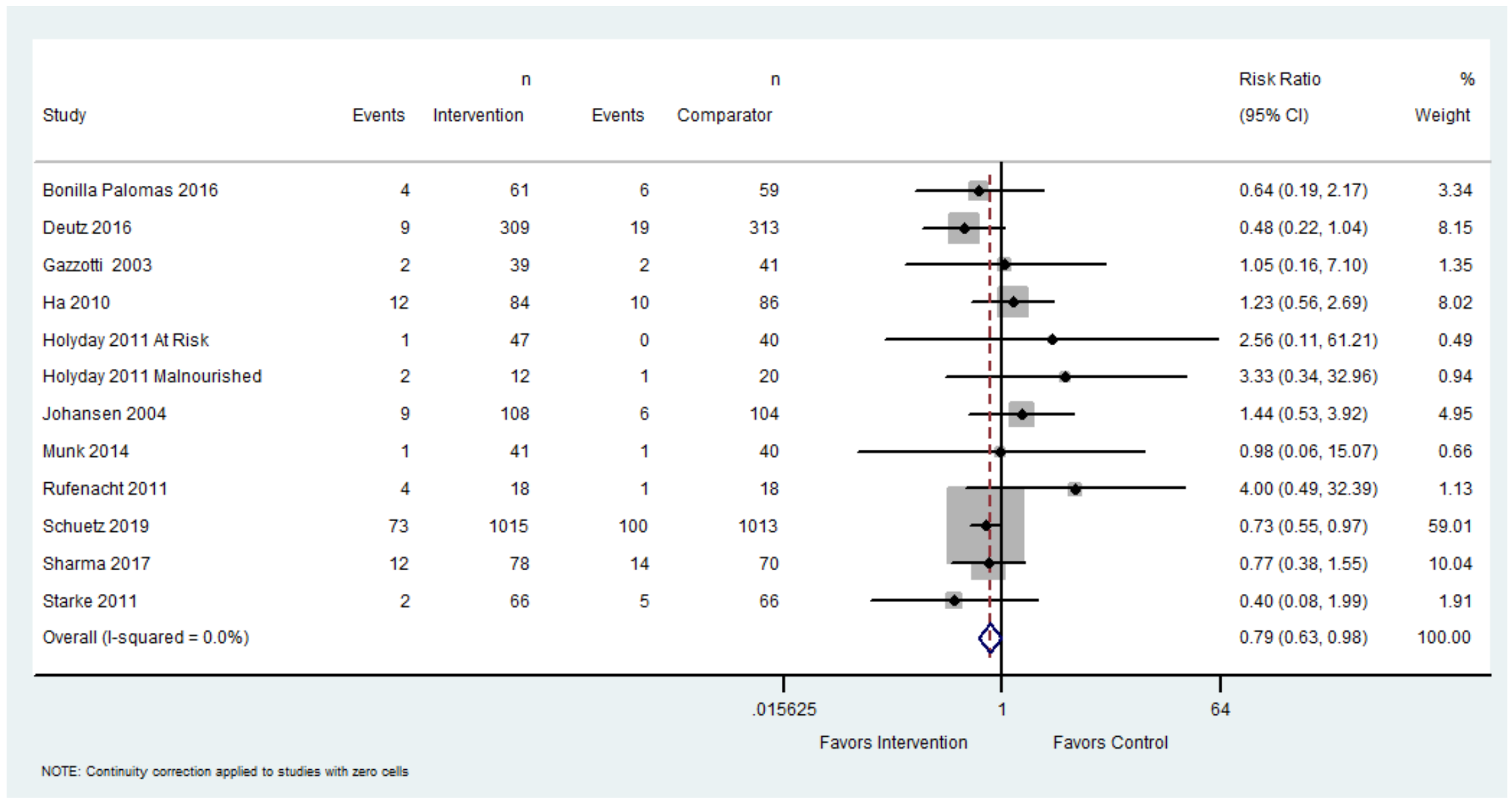


Figure G-2. Effect of hospital-initiated interventions on mortality, subgroup intervention type

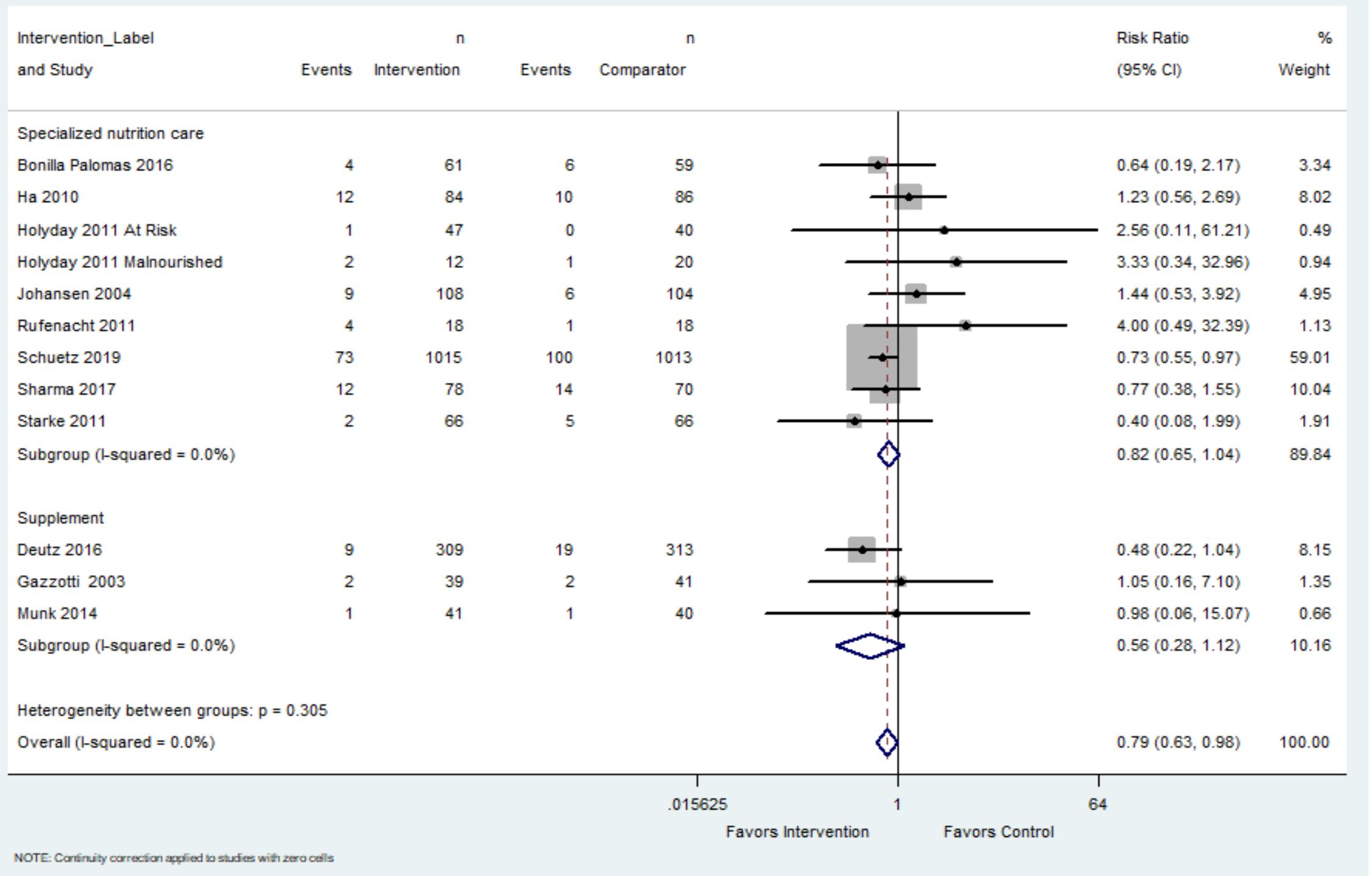


Figure G-3. Effect of hospital-initiated interventions on mortality, subgroup malnutrition status

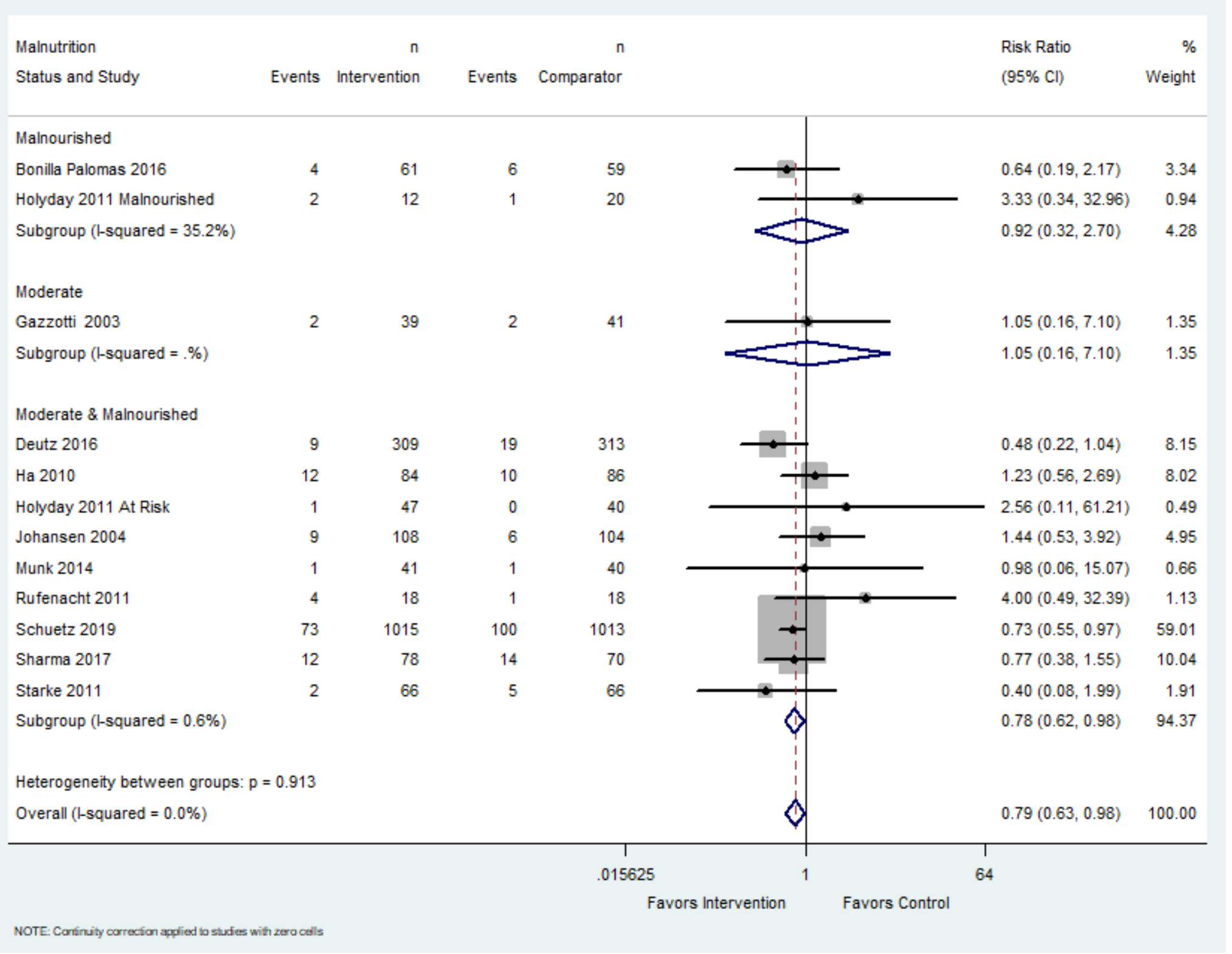


Figure G-4. Effect of hospital-initiated interventions on mortality, subgroup followup time

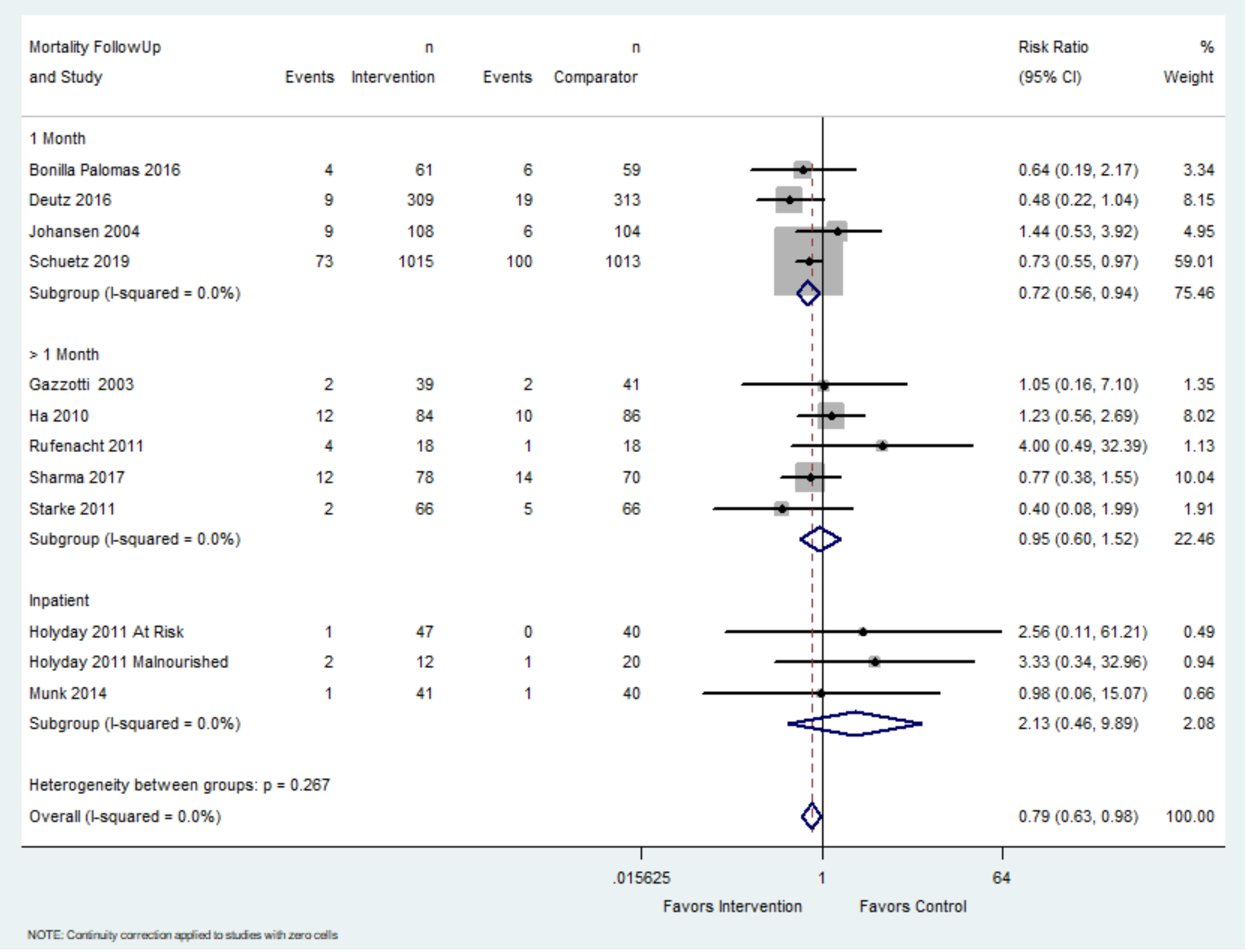


Figure G-5. Effect of hospital-initiated interventions on length of stay, all studies

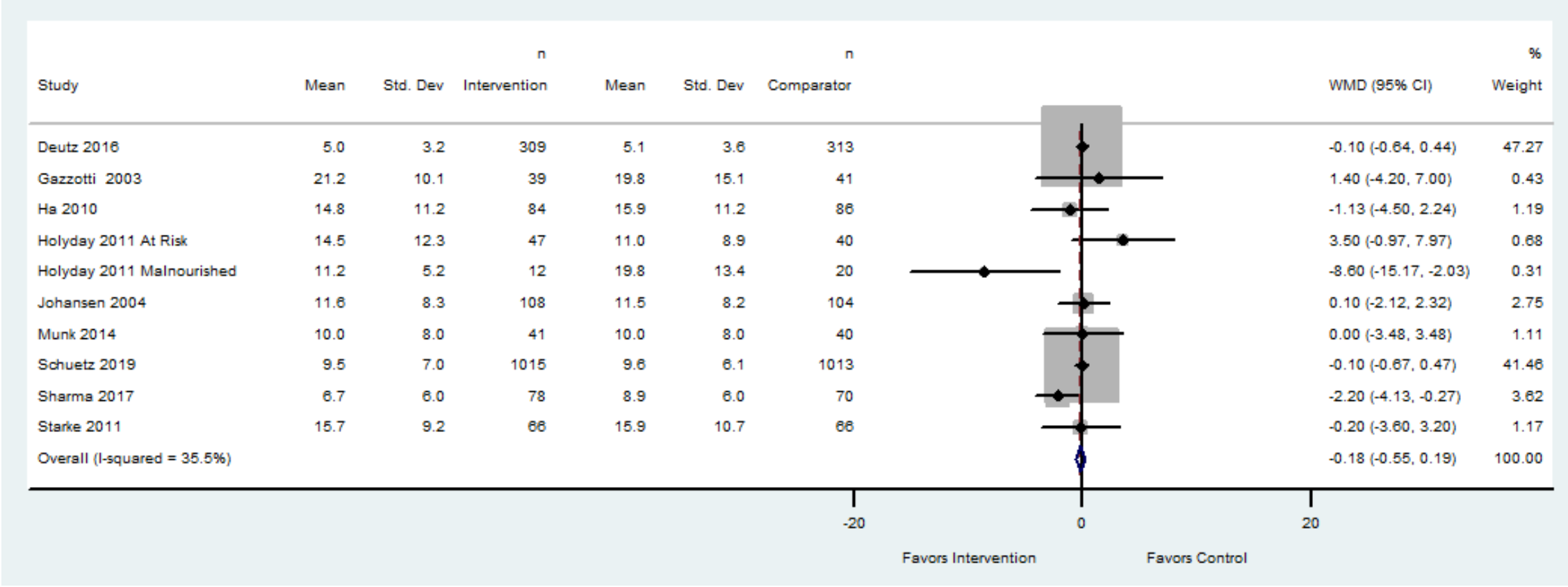


Figure G-6. Effect of hospital-initiated interventions on length of stay, subgroup type of intervention

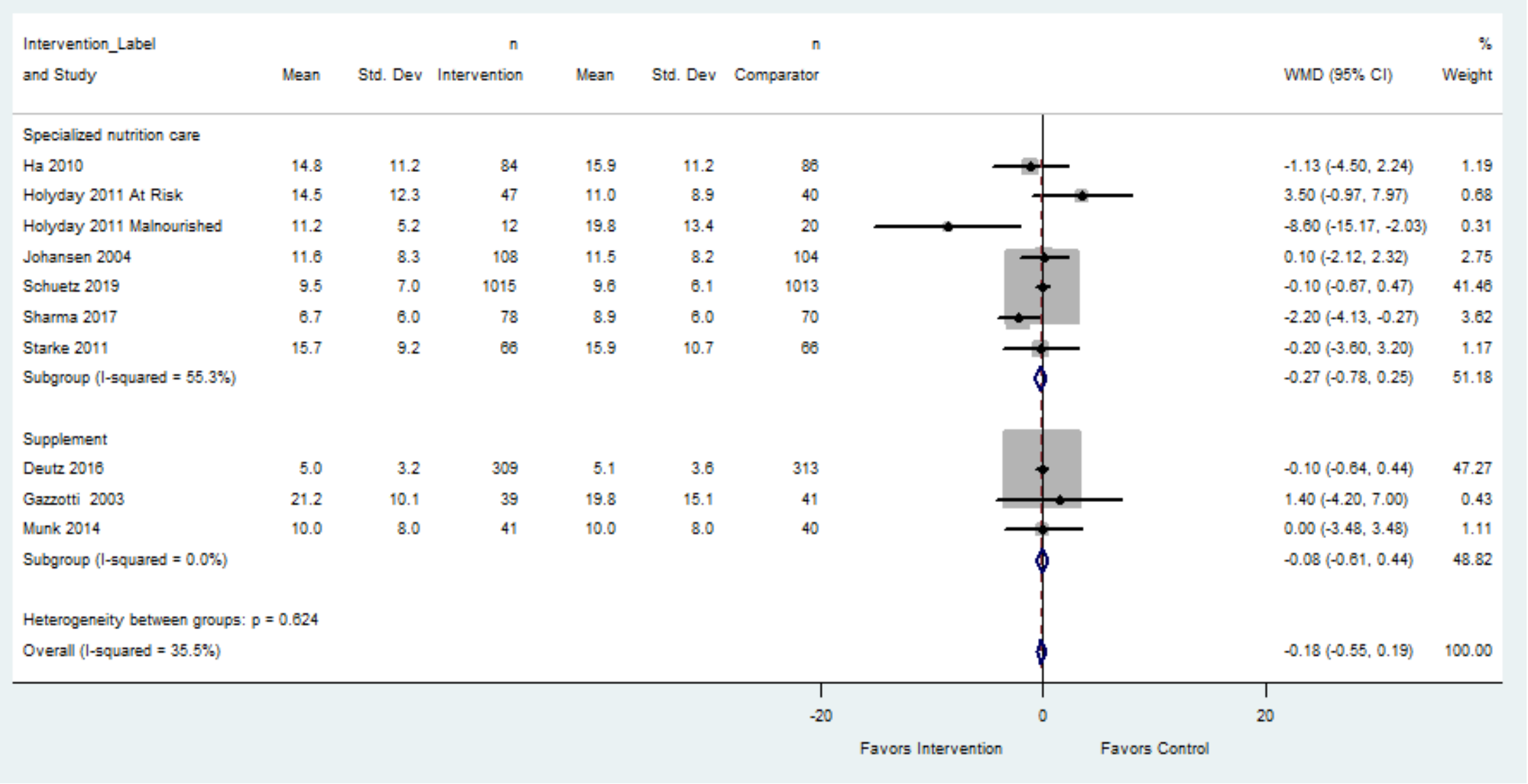


Figure G-7. Effect of hospital-initiated interventions on length of stay, subgroup malnutrition status

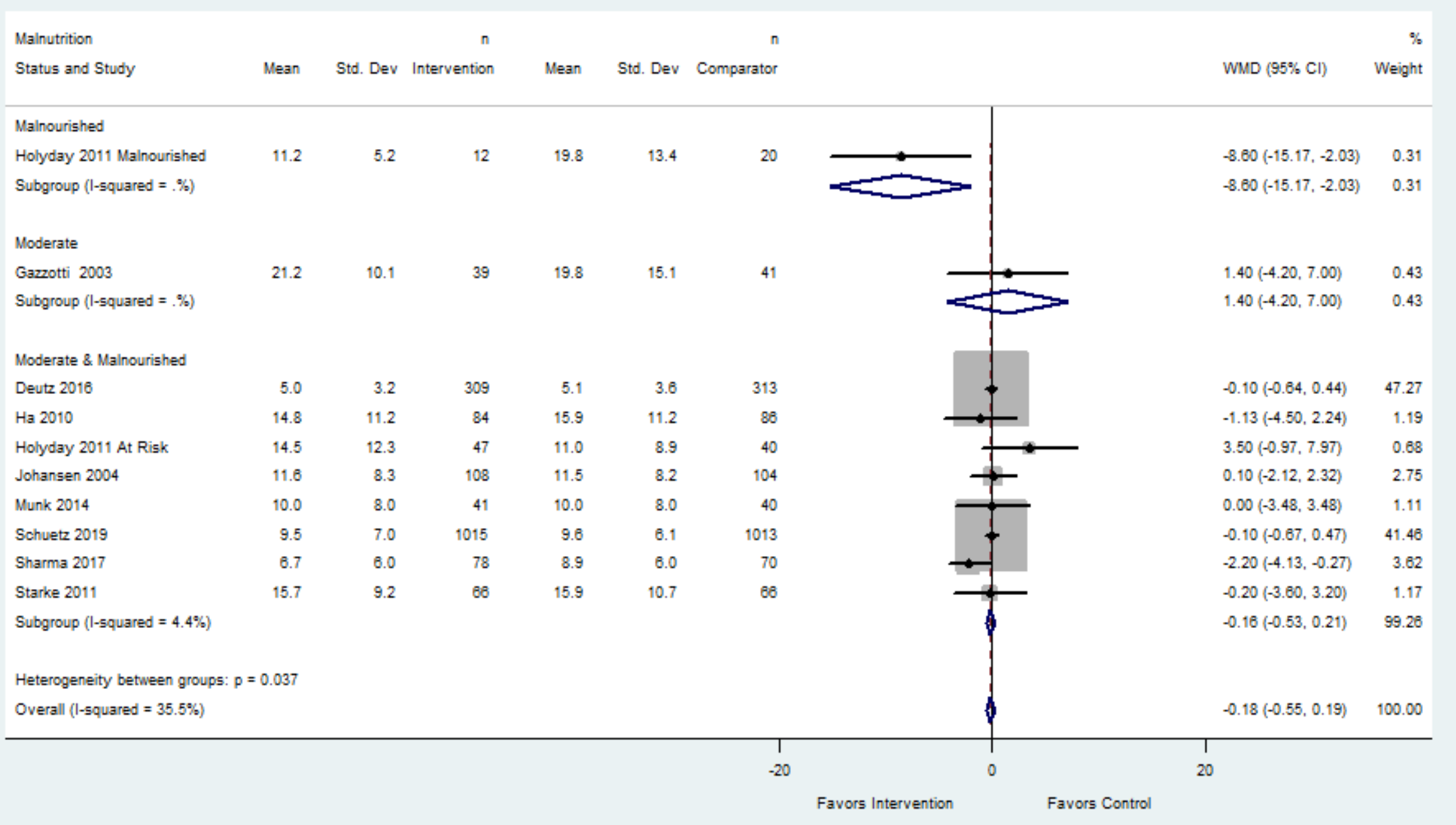


Figure G-8. Effect of hospital-initiated interventions on readmissions, all studies

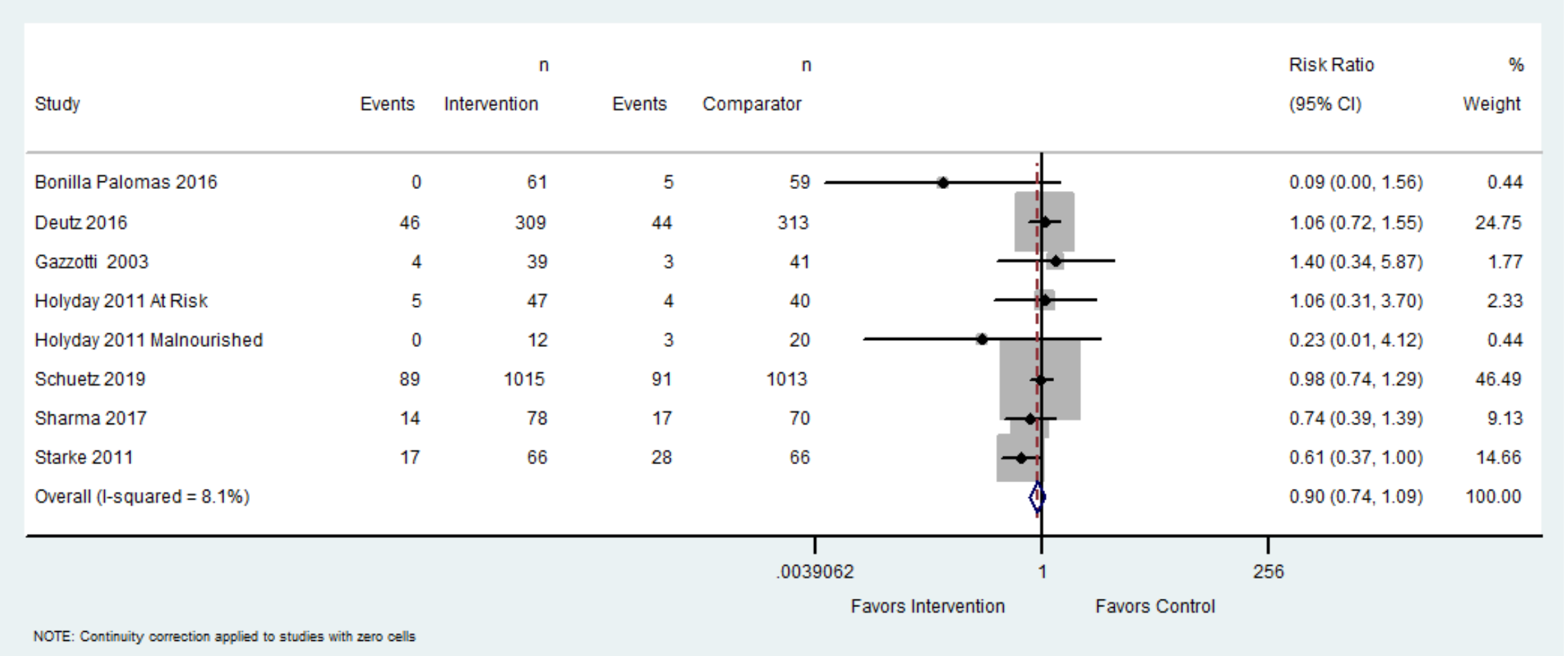


Figure G-9. Effect of hospital-initiated interventions on readmissions, subgroup type of intervention

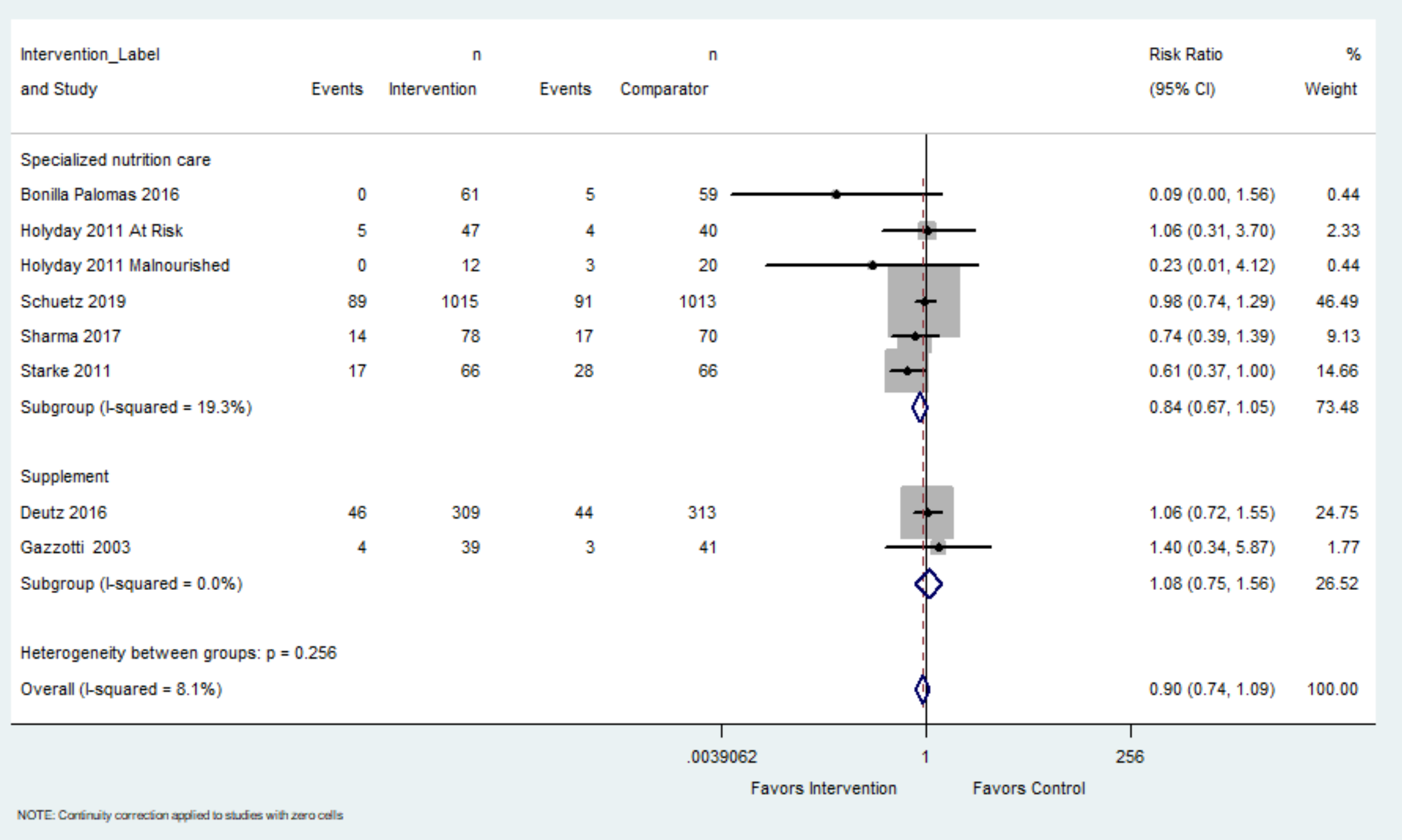


Figure G-10. Effect of hospital-initiated interventions on readmissions, subgroup malnutrition status

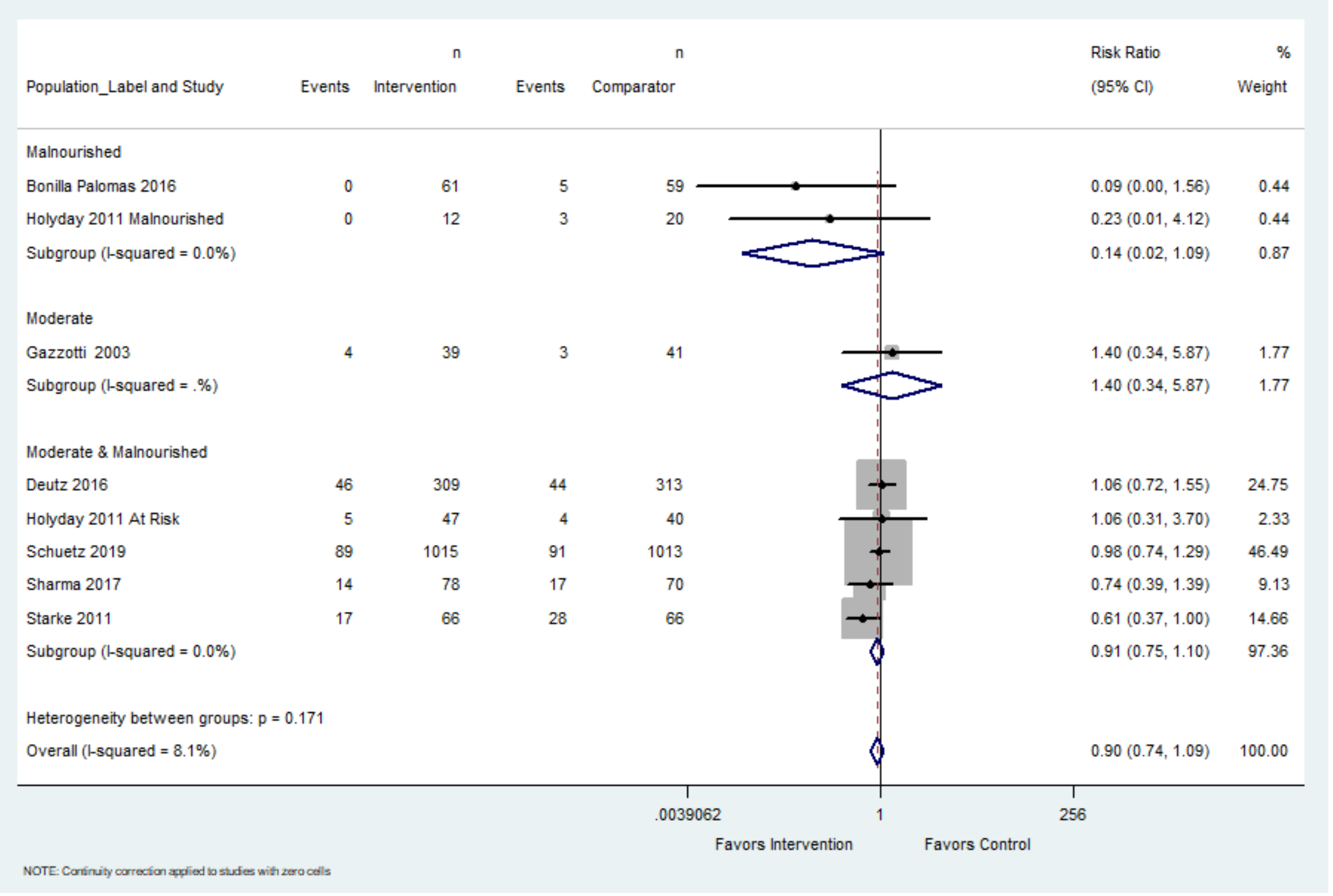


Figure G-11. Effect of hospital-initiated interventions on readmissions, subgroup follow-up time

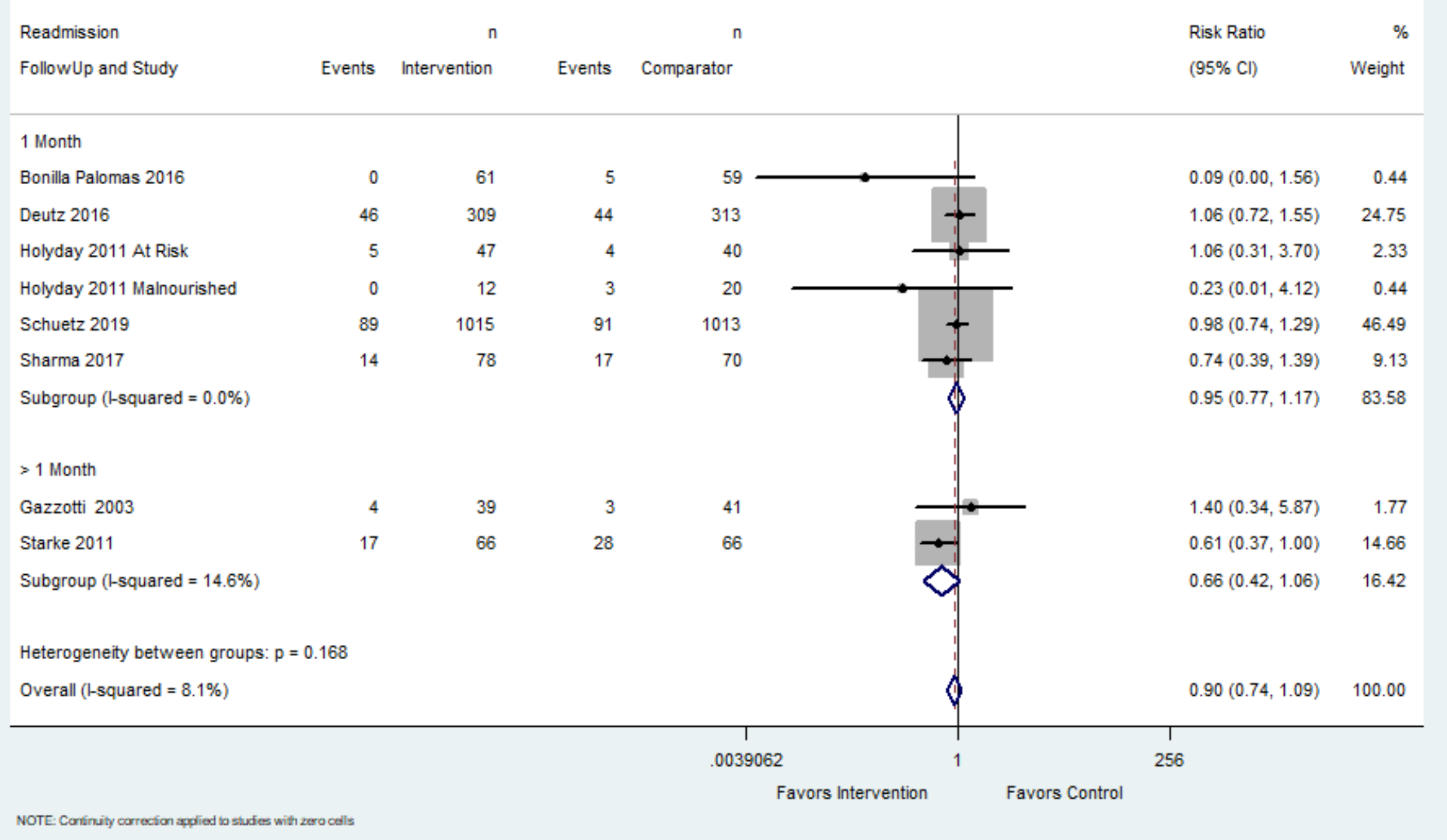


Figure G-12. Effect of hospital-initiated interventions on hospital acquired conditions, all studies

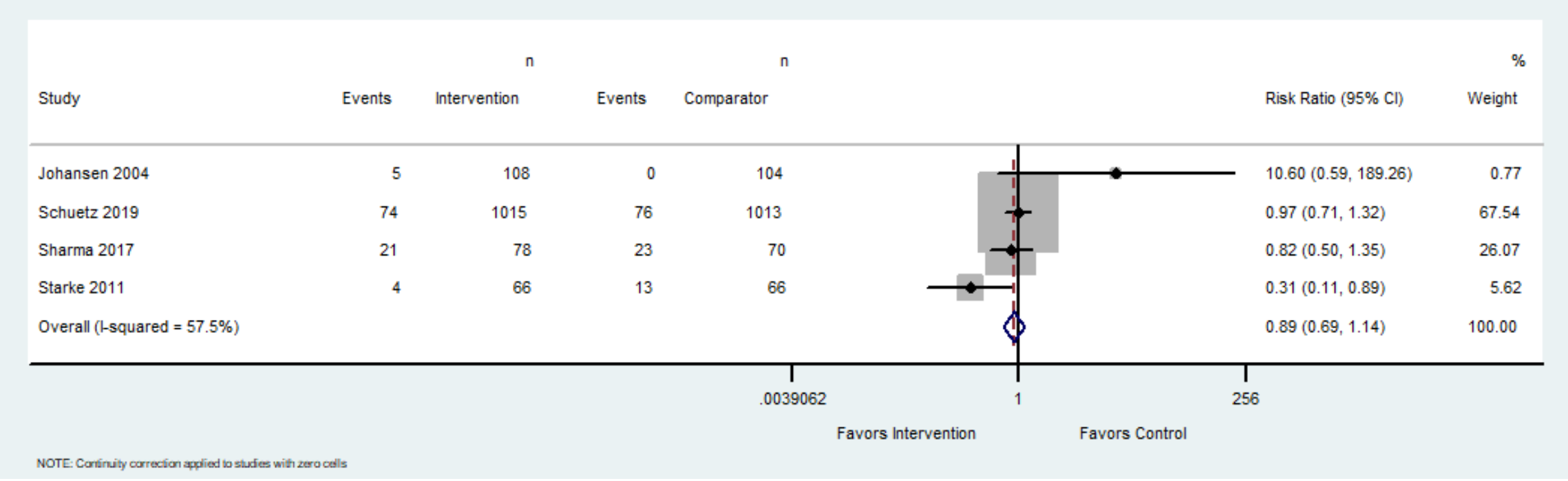


Figure G-13. Effect of hospital-initiated interventions on quality of life, subgroup Quality of Life Measurement Tool

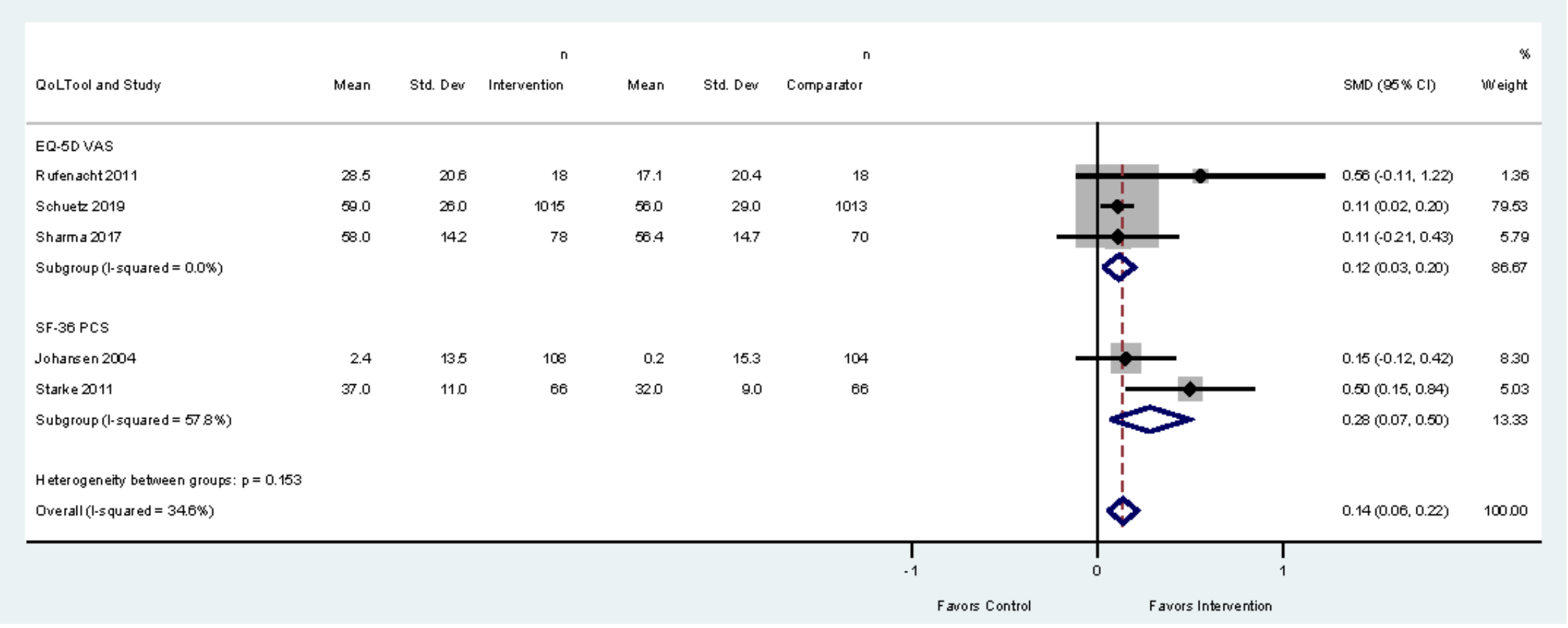




\section{Appendix H. Additional Information for KQ 1}

Table H-1. Studies included in systematic reviews

\begin{tabular}{|c|c|c|c|c|c|}
\hline DiJkink 2020 & Ney 2019 & Muscaritoli 2017 & Lew 2016 & Lin 2016 & Gupta 2011 \\
\hline $\begin{array}{l}\text { Wilson et al. 2019 } \\
\text { Wilson et al. 2019 } \\
\text { Wintermeyer } \\
\text { et al. } 2019 \\
\text { Ihle et al. } 2017 \\
\text { Müller et al. } 2017 \\
\text { Goisser et al. } 2015 \\
\text { Chakravarty et al. } 2013 \\
\text { Banks et al. } 2010 \\
\text { Dhandapani et al. } 2007 \\
\text { Goiburu et al. } 2006 \\
\text { Compan et al. } 1999 \\
\text { McClave et al. } 1992 \\
\text { Kaufman et al. } 1987\end{array}$ & $\begin{array}{l}\text { Ciocirlan } 2017 \\
\text { Nunes } 2017 \\
\text { Bakshi } 2016 \\
\text { Gaikwad } 2016 \\
\text { Yosry } 2014 \\
\text { Alveras } 2005 \\
\text { Stephenson } 2001 \\
\text { Pikul } 1994\end{array}$ & $\begin{array}{l}\text { Agarwal et al. } 2013 \\
\text { Zapatero et al. } 2013 \\
\text { Koren-Hakim et al. } 2012 \\
\text { Lim et al. } 2012 \\
\text { Zapatero et al. } 2012 \\
\text { Aziz et al. } 2011 \\
\text { Benedik et al. } 2011 \\
\text { Mudge et al. } 2011 \\
\text { Hamaguchi et al. } 2010 \\
\text { Steer et al. } 2010 \\
\text { Lobo Tamer et al. } 2009 \\
\text { Planas et al. } 2004 \\
\text { Vecchiarino et al. } 2004 \\
\text { Thomas et al. } 2002 \\
\text { Chima et al. } 1997\end{array}$ & $\begin{array}{l}\text { Coltman et al. } 2015 \\
\text { Fontes et al. } 2014 \\
\text { Tripathy et al. } 2014 \\
\text { Chakravarty et al. } 2013 \\
\text { Lomivorotov et al. } 2013 \\
\text { Sheean et al. } 2013 \\
\text { Caporossi et al. } 2012 \\
\text { Sheean et al. } 2012 \\
\text { van Venrooij et al. } 2011 \\
\text { Merli et al. 2010 } \\
\text { Peterson et al. } 2010 \\
\text { Sheean et al. } 2010 \\
\text { Terekeci et al. } 2009 \\
\text { de Luis et al. } 2008 \\
\text { Guimaraes et al. } 2008 \\
\text { Küçükardali et al. } 2008 \\
\text { Sungurtekin et al. } 2008 \\
\text { Banks et al. } 2007 \\
\text { Penie et al. } 2005 \\
\text { Schlossmcher et al. } 2002\end{array}$ & $\begin{array}{l}\text { Kaneko et al. } 2015 \\
\text { Suzuki et al. } 2015 \\
\text { Gastelurrutia et al. } 2014 \\
\text { Gouya et al. } 2014 \\
\text { Tevik et al. } 2014 \\
\text { Yost et al. } 2014 \\
\text { Aggarwal et al. } 2013 \\
\text { Kinugasa et al. } 2013 \\
\text { Narumi et al. } 2013 \\
\text { Sargento et al. } 2013 \\
\text { Son et al. } 2013 \\
\text { Al-Najjar et al. } 2012 \\
\text { Aziz et al. 2011 } \\
\text { Bonilla-Palomas et al. } 2011 \\
\text { Gastelurrutia et al. } 2011 \\
\text { Veloso et al. } 2006\end{array}$ & $\begin{array}{l}\text { Laky, } 2010 \\
\text { Wu, } 2010 \\
\text { Wu, 2009 } \\
\text { Wakahara, } 2007 \\
\text { Horsley, } 2005 \\
\text { Shirodkar, } 2005 \\
\text { Bauer, 2002 } \\
\text { Ulander, } 1998\end{array}$ \\
\hline
\end{tabular}


Table H-2. Individual studies not included in existing SR for KQ 1

\begin{tabular}{|c|c|c|}
\hline Reference & Nutritional Status Tool & Medical Condition \\
\hline Acehan S. et al. $2020^{91}$ & NRS & Critically ill \\
\hline Bedock et al. $2020^{92}$ & GLIM & COVID-19 \\
\hline Brascher et al. $2020^{93}$ & NUTRIC & Hospitalized patients \\
\hline Burgos et al. $2020^{94}$ & NRS & Chronic disease \\
\hline Dou et al. $2020^{95}$ & NRS & Cancer \\
\hline Galindo et al. $2020^{96}$ & GLIM & Critically ill \\
\hline Han et al. $2020^{97}$ & MUST & Hip fracture \\
\hline Hirose et al. $2020^{98}$ & GNRI & Heart failure \\
\hline Kaddoura et al. $2020^{99}$ & PG-SGA & Critically ill \\
\hline Karim et al. $2020^{100}$ & Not reported in abstract & Heart transplant \\
\hline Karin et al. $2020^{101}$ & PG-SGA, MUST & Cancer \\
\hline Kootaka et al. $2020^{102}$ & GLIM & CVD \\
\hline Machado et al. $2020^{103}$ & NUTRIC, NRS & Critically ill \\
\hline Matsumoto et al. $2020^{104}$ & NRS, GLIM & Critically ill \\
\hline Maurer et al. $2020^{105}$ & NRS & Critically ill \\
\hline Pratt et al. $2020^{106}$ & Not reported in abstract & Hospitalized patients \\
\hline Tao et al. $2020^{107}$ & ESPEN & Older adults \\
\hline Toledo et al. $2020^{108}$ & NUTRIC & Critically ill \\
\hline Tonet et al. $2020^{109}$ & MNA-SF & CVD \\
\hline Trestini I. et al. $2020^{110}$ & NRS & Cancer \\
\hline Yilmiz et al. $2020^{111}$ & NRS, GLIM & Cancer \\
\hline Zhao et al. $2020^{112}$ & GNRI & Older adults \\
\hline Nishi et al. $2019^{113}$ & GNRI & Heart failure \\
\hline Abd-Elraheem M. et al. $2019^{114}$ & MUST & Hospitalized patients \\
\hline Almasaudi et al. $2019^{115}$ & MUST & Cancer \\
\hline Chien et al. $2019^{116}$ & PNI, CONUT, GNRI & CVD \\
\hline Contreras-Bolovar et al. $2019^{117}$ & SGA & Cancer \\
\hline Gonzalez et al. $2019^{118}$ & NUTRIC & Critically ill \\
\hline Gottschall C et al. $2019^{119}$ & MUST & Hospitalized patients \\
\hline Hirose EY. et al. $2019^{120}$ & SGA & Stem cell transplant \\
\hline Inoue T et al. $2019^{121}$ & MNA-SF, MUST, GNRI, NRS & Hip fracture \\
\hline Komici K. et al. $2019^{122}$ & MNA & CVD \\
\hline Minamisawa M et al. $2019^{123}$ & GNRI & CVD \\
\hline Muller et al. $2019^{124}$ & NRS & CKD \\
\hline Ruiz AJ et al. $2019^{125}$ & MST & Cardio-Pulmonary conditions \\
\hline Sauer et al. $2019^{126}$ & MST & Hospitalized patients \\
\hline Subwongcharoen S. et al. $2019^{127}$ & SGA & Cancer \\
\hline Viana MV et al. $2019^{128}$ & NRS & Critically ill \\
\hline Ceniccola GD. et al. $2018^{129}$ & AND-ASPEN & Critically ill \\
\hline Chen WZ. et al. $2018^{130}$ & Not reported in abstract & Cancer \\
\hline Dent E et al. $2018^{131}$ & MNA, MNA-SF & Older adults \\
\hline Marcadenti A. et al. $2018^{132}$ & NRE-2017; Nurtritional Risk in Emergency & Hospitalized patients \\
\hline Morris N. et al. $2018^{133}$ & SGA & Hospitalized patients \\
\hline Ramos R. et al. $2018^{134}$ & NRI & Cancer \\
\hline Rondel et al. $2018^{135}$ & ESPEN & Hospitalized patients \\
\hline Borek P. et al. $2017^{136}$ & NRS & CKD \\
\hline Guerra RS. et al. $2017^{137}$ & SGA & Hospitalized patients \\
\hline
\end{tabular}




\begin{tabular}{|c|c|c|}
\hline Reference & Nutritional Status Tool & Medical Condition \\
\hline Inoue T. et al. $2017^{138}$ & MNA-SF & Hip fracture \\
\hline Kalaiselvan MS et al. $2017^{139}$ & NUTRIC & Critically ill \\
\hline Kirushnan BB et al. $2017^{140}$ & SGA & Dialysis \\
\hline Leiva Badosa et al. $2017^{141}$ & MUST, SNAQ & Critically ill \\
\hline Martucci RB et al. $2017^{142}$ & MNA-SF & Cancer \\
\hline Pierik VD et al. $2017^{143}$ & SNAQ & Older adults \\
\hline Potyraa P. et al. $2017^{144}$ & NRS & Cancer \\
\hline Rabito El. Et al. $2017^{145}$ & MUST, MST, SNAQ, NRS & Hospitalized patients \\
\hline Salomon du Mont et al. $2017^{146}$ & NRI & Critically ill \\
\hline Sharma Y et al. $2017^{147}$ & PG-SGA & Hospitalized patients \\
\hline Soderstrom L. et al. $2017^{148}$ & MNA & Older adults \\
\hline Yun T. et al. $2017^{149}$ & SGA & Hospitalized patients \\
\hline Allard JP et al. $2016^{150}$ & SGA & Hospitalized patients \\
\hline Bakshi N. et al. $2016^{151}$ & SGA & Liver transplant \\
\hline Gau BR et al. $2016^{152}$ & MNA & Diabetes \\
\hline Guerra RS et al. $2016^{153}$ & $\begin{array}{l}\text { NRS-2002, MUST, AND/ASPEN, PG- } \\
\text { SGA }\end{array}$ & Hospitalized patients \\
\hline Gultekin A. et al. $2016^{154}$ & SGA, NRS & Hospitalized patients \\
\hline Koren-Hakim T. et al. $2916^{155}$ & MNA-SF, MUST, NRS & Hip fracture \\
\hline Kruizenga H. et al. $2016^{156}$ & SNAQ, MUST & Hospitalized patients \\
\hline Maasberg S et al. $2016^{157}$ & Not reported in abstract & Cancer \\
\hline Sanz-Paris A. et al. $2016^{158}$ & ESPEN & Diabetes \\
\hline Tan SK et al. $2016^{159}$ & SGA & Dialysis \\
\hline van Wissen J. et al. $2016^{160}$ & MNA & Hip fracture \\
\hline Coltman A. et al. $2015^{161}$ & SGA, NUTRIC & Critically ill \\
\hline Cui J. et al. $2015^{162}$ & NRS & COPD \\
\hline Dent E. et al. $2015^{163}$ & MNA, MNA-SF, GNRI & Older adults \\
\hline Felder S. et al. $2015^{164}$ & NRS & Critically ill \\
\hline Grass F. et al. $2015^{165}$ & NRS & Hospitalized patients \\
\hline Guerra RS et al. $2015^{166}$ & NRS, PG-SGA, MUST & Hospitalized patients \\
\hline Jeejeebhoy $\mathrm{KN}$ et al. $2015^{167}$ & SGA, NRS & Hospitalized patients \\
\hline Leandro-Merhi VA et al. $2015^{168}$ & NRS, SGA & Cancer \\
\hline Rodrigues CS et al. $2015^{169}$ & PG-SGA & Cancer \\
\hline Simpson F \& Doig GS. 2015 & Not reported in abstract & Critically ill \\
\hline Sohrabi Z. et al. $2015^{171}$ & SGA & Dialysis \\
\hline Bell JJ et al. $2014^{172}$ & MNA-SF & Hip fracture \\
\hline Chermesh I. et al. $2014^{173}$ & MUST & Cardio-Pulmonary conditions \\
\hline Cui H. et al. $2014^{174}$ & PG-SGA & Cancer \\
\hline de Mendonoa Soares BL et al. $2014^{175}$ & NRS & Cancer \\
\hline Huang TH et al. $2014^{176}$ & PG-SGA & Critically ill \\
\hline Mendes J. et al. $2014^{177}$ & PG-SGA, NRS & Cancer \\
\hline Schrader E. et al. $2014^{178}$ & MNA & Older adults \\
\hline Soderstrom L. et al. $2014^{179}$ & MNA & Older adults \\
\hline Almeida Al et al. $2013^{180}$ & NRS, MUST, SGA & Hospitalized patients \\
\hline Holst M. et al. $2013^{181}$ & MNA, MUST & Older adults \\
\hline Lee JS et al. $2013^{182}$ & GNRI & Older adults \\
\hline Rasheed S \& Woods RT. 2013 ${ }^{183}$ & NRI & Older adults \\
\hline Ulltang M. et al. $2013^{184}$ & MST, SGA & Hospitalized patients \\
\hline Zhang SS et al. $2013^{185}$ & SGA & Diabetes \\
\hline
\end{tabular}




\begin{tabular}{|c|c|c|}
\hline Reference & Nutritional Status Tool & Medical Condition \\
\hline Charlton K. et al. $2012^{186}$ & MNA & Hospitalized patients \\
\hline Gamaletsou MN et al. $2012^{187}$ & GNRI & Hospitalized patients \\
\hline Komlanvi K. et al. $2012^{188}$ & MNA, GNRI & Older adults \\
\hline Pavic T. et al. $2012^{189}$ & NRS & Hospitalized patients \\
\hline Teiusanu A. et al. $2012^{190}$ & SGA & Cirrhosis \\
\hline Chermesh I. et al. $2011^{191}$ & MUST & Hospitalized patients \\
\hline Karl A. et al. $2011^{192}$ & NRS & Hospitalized patients \\
\hline Kuseolu Z. et al. $2011^{193}$ & NRS & Hospitalized patients \\
\hline Hafsteinsdottir TB et al. $2010^{194}$ & MNA & Neurological \\
\hline Merli M. et al. $2010^{195}$ & SGA & Cirrhosis \\
\hline Zamora RJ et al. $2010^{196}$ & SGA & Older adults \\
\hline Fiedler R. et al. $2009^{197}$ & SGA, NRS & Dialysis \\
\hline Oliveira MR et al. $2009^{198}$ & MNA & Older adults \\
\hline Ozkalkanli MY et al. $2009^{199}$ & NRS, SGA & Ortho surgery \\
\hline Bin J. et al. $2008^{200}$ & NRS & Hospitalized patients \\
\hline Casariego AV \& Fernandez MJ $2008^{201}$ & SGA & Hospitalized patients \\
\hline Henderson S. et al. $2008^{202}$ & MUST & Older adults \\
\hline Kukardali Y. et al. $2008^{203}$ & NRS & Hospitalized patients \\
\hline Sungurtekin H. et al. $2008^{204}$ & SGA & Critically ill \\
\hline Gurreebun F. et al. $2007^{205}$ & SGA & Dialysis \\
\hline Wakahara T. et al. $2007^{206}$ & SGA & Digestive disease \\
\hline Kruizenga HM et al. $2006^{207}$ & SNAQ & Hospitalized patients \\
\hline Kyle UG et al. $2006^{208}$ & NRI, MUST, NRS, SGA & Hospitalized patients \\
\hline Stratton RJ et al. $2006^{209}$ & MUST & Older adults \\
\hline Bouillanne O. et al. $2005^{210}$ & GNRI & Older adults \\
\hline Kagansky N. et al. $2005^{211}$ & MNA & Older adults \\
\hline Norman K. et al. $2005^{212}$ & SGA & Hospitalized patients \\
\hline Pepersack T. $2005^{213}$ & Not reported in abstract & Older adults \\
\hline Thomas DR et al. $2005^{214}$ & Not reported in abstract & Hospitalized patients \\
\hline Kyle UG et al. $2004^{215}$ & NRI & Hospitalized patients \\
\hline Pichard C. et al. $2004^{216}$ & SGA & Hospitalized patients \\
\hline Visvanathan R. et al. $2004^{217}$ & MNA & Older adults \\
\hline Bauer J. et al. $2002^{218}$ & PG-SGA & Cancer \\
\hline Persson MD et al. $2002^{219}$ & SGA, MNA & Older adults \\
\hline Kalantar-Zadeh et al. $2001^{220}$ & SGA & Dialysis \\
\hline Laws RA et al. 221 & SGA & Dialysis \\
\hline Santoso JT et al. $2000^{222}$ & Prognostic Nutritional Index & Cancer \\
\hline
\end{tabular}




\section{Appendix I. Appendix References}

1. Owens D, Lohr K, Atkins D, et al. Grading the strength of a body of evidence when comparing medical interventions. In: Agency for Healthcare Research and Quality (AHRQ). Methods guide for comparative effectiveness reviews. Rockville (MD): Agency for Healthcare Research and Quality (AHRQ); 2009 Jul. https://effectivehealthcare.ahrq.gov/products/ methods-guidance-grading-strength/methods.

2. Chalmers I, Adams M, Dickersin K, et al. A cohort study of summary reports of controlled trials. JAMA. 1990 Mar 9;263(10):1401-5. PMID: 2304219.

3. Neinstein LS. A review of Society for Adolescent Medicine abstracts and Journal of Adolescent Health Care articles. J Adolesc Health Care. 1987 Mar;8(2):198-203. PMID: 3818406 .

4. Dundar Y, Dodd S, Williamson P, et al. Case study of the comparison of data from conference abstracts and full-text articles in health technology assessment of rapidly evolving technologies: does it make a difference? Int J Technol Assess Health Care. 2006 Jul;22(3):288-94. PMID: 16984055.

5. De Bellefeuille C, Morrison CA, Tannock IF. The fate of abstracts submitted to a cancer meeting: factors which influence presentation and subsequent publication. Ann Oncol. 1992 Mar;3(3):187-91. PMID: 1586615.

6. Scherer RW, Meerpohl JJ, Pfeifer N, et al. Full publication of results initially presented in abstracts. In: Cochrane Library [Cochrane methodology review]. Issue 11. John Wiley \& Sons, Inc.; 2018 Nov 20 [accessed 2020 Aug 14]. Available:

https://doi.org/10.1002/14651858.MR000005. pub4. PMID: 30480762.

7. Yentis SM, Campbell FA, Lerman J. Publication of abstracts presented at anaesthesia meetings. Can J Anaesth. 1993 Jul;40(7):632-4. PMID: 8403137.

8. Burgel CF, Teixeira PP, Leites GM, et al. Concurrent and predictive validity of ANDASPEN malnutrition consensus is satisfactory in hospitalized patients: a longitudinal study. J Parenter Enteral Nutr. 2020 Aug;Epub ahead of print. http://dx.doi.org/10.1002/jpen.1980. PMID: 32738153.
9. Cattani A, Eckert IC, Brito JE, et al. Nutritional risk in critically ill patients: how it is assessed, its prevalence and prognostic value: a systematic review. Nutr Rev. 2020 Dec;78(12):1052-68. http://dx.doi.org/10.1093/nutrit/nuaa031. PMID: 32529226.

10. Habaybeh D, de Moraes MB, Slee A, et al. Nutritional interventions for heart failure patients who are malnourished or at risk of malnutrition or cachexia: a systematic review and meta-analysis. Heart Fail Rev. 2020 Mar 2;Epub ahead of print. http://dx.doi.org/10.1007/s10741-020-099379. PMID: 32124164.

11. Hsueh SW, Liu KH, Hung CY, et al. Predicting postoperative events in patients with gastric cancer: a comparison of five nutrition assessment tools. In Vivo. 2020 Sep;34(5):2803-9. http://dx.doi.org/10.21873/invivo.12106. PMID: 32871818.

12. Jantharapattana K, Orapipatpong O. Efficacy of EPA-enriched supplement compared with standard formula on body weight changes in malnourished patients with head and neck cancer undergone surgery: a randomized study. Head Neck. 2020 Feb;42(2):188-97. http://dx.doi.org/10.1002/hed.25987. PMID: 31647147.

13. Lima J, Teixeira PP, Eckert IDC, et al. Decline of nutritional status in the first week of hospitalization predicts longer length of stay and hospital readmission during six-month follow-up. Br J Nutr. 2020 Sep 3;1-8. Epub ahead of print.

http://dx.doi.org/10.1017/S0007114520003451 . PMID: 32878650.

14. Matheson EM, Nelson JL, Baggs GE, et al. Specialized oral nutritional supplement (ONS) improves handgrip strength in hospitalized, malnourished older patients with cardiovascular and pulmonary disease: a randomized clinical trial. Clin Nutr. 2020 Sep 5;S0261-5614(20):30451-9. http://dx.doi.org/10.1016/j.clnu.2020.08.035. PMID: 32943241. 
15. Torbahn G, Strauss T, Sieber CC, et al. Nutritional status according to the mini nutritional assessment (MNA) ${ }^{\circledR}$ as potential prognostic factor for health and treatment outcomes in patients with cancer - a systematic review. BMC Cancer. 2020 Jun 26;20(1):594. http://dx.doi.org/10.1186/s12885-020-070524. PMID: 32586289.

16. Valladares AF, Kilgore KM, Partridge J, et al. How a malnutrition quality improvement initiative furthers malnutrition measurement and care: results from a hospital learning collaborative. J Parenter Enteral Nutr. 2020 Apr 13;Epub ahead of print. http://dx.doi.org/10.1002/jpen.1833. PMID: 32282948 .

17. Xie H, Tang S, Wei L, et al. Geriatric nutritional risk index as a predictor of complications and long-term outcomes in patients with gastrointestinal malignancy: a systematic review and meta-analysis. Cancer Cell Int. 2020 Oct 31;20(1):530. http://dx.doi.org/10.1186/s12935-020-016287. PMID: 33292289.

18. Becker L, Volkert D, Christian Sieber C, et al. Predictability of a modified mini- nutritionalassessment version on six-month and one-year mortality in hospitalized geriatric patients: a comparative analysis. Sci Rep. 2019 Jun 21;9(1):9064. http://dx.doi.org/10.1038/s41598-019-45452‥ PMID: 31227778.

19. Gomes F, Baumgartner A, Bounoure L, et al. Association of nutritional support with clinical outcomes among medical inpatients who are malnourished or at nutritional risk: an updated systematic review and meta-analysis. JAMA Netw Open. 2019 Nov;2(11):e1915138. http://dx.doi.org/10.1001/jamanetworkopen.20 19.15138. PMID: 31747030.

20. Lovesley D, Parasuraman R, Ramamurthy A. Combating hospital malnutrition: dietitian-led quality improvement initiative. Clin Nutr ESPEN. 2019 Apr;30:19-25. http://dx.doi.org/10.1016/j.clnesp.2019.02.011 . PMID: 30904220.
21. Fuentes Padilla P, Martínez G, Vernooij RWM, et al. Early enteral nutrition (within 48 hours) versus delayed enteral nutrition (after 48 hours) with or without supplemental parenteral nutrition in critically ill adults. Cochrane Database Syst Rev. 2019 Oct 31;(10):CD012340. http://dx.doi.org/10.1002/14651858.CD01234 0.pub2. PMID: 31684690.

22. Rinninella E, Fagotti A, Cintoni M, et al. Nutritional interventions to improve clinical outcomes in ovarian cancer: a systematic review of randomized controlled trials. Nutrients. 2019 Jun 21;11(6):1404. http://dx.doi.org/10.3390/nu11061404. PMID: 31234395 .

23. Thomas J, Kaambwa B, Delaney C, et al. An evaluation of the validity of nutrition screening and assessment tools in patients admitted to a vascular surgery unit. Br J Nutr. 2019 Sep 28;122(6):689-97. http://dx.doi.org/10.1017/S0007114519001442 . PMID: 31256768.

24. Yang PH, Lin MC, Liu YY, et al. Effect of nutritional intervention programs on nutritional status and readmission rate in malnourished older adults with pneumonia: a randomized control trial. Int J Environ Res Public Health. 2019 Nov 27;16(23):1-12. http://dx.doi.org/10.3390/ijerph16234758. PMID: 31783672.

25. Yu K, Zheng X, Wang G, et al. Immunonutrition vs standard nutrition for cancer patients: a systematic review and metaanalysis (part 1). J Parenter Enteral Nutr. 2020 Jul;44(5):742-67. http://dx.doi.org/10.1002/jpen.1736. PMID: 31709584.

26. Malafarina V, Reginster JY, Cabrerizo S, et al. Nutritional status and nutritional treatment are related to outcomes and mortality in older adults with hip fracture. Nutrients. 2018 Apr 30;10(5):555. http://dx.doi.org/10.3390/nu10050555. PMID: 29710860.

27. Ortíz-Reyes LA, Castillo-Martínez L, LupiánAngulo AI, et al. Increased efficacy and safety of enteral nutrition support with a protocol (ASNET) in noncritical patients: a randomized controlled trial. J Acad Nutr Diet. 2018 Jan;118(1):52-61. http://dx.doi.org/10.1016/j.jand.2017.09.020. PMID: 29274643. 
28. Perman MI, Ciapponi A, Franco JVA, et al. Prescribed hypocaloric nutrition support for critically-ill adults. Cochrane Database Syst Rev. 2018 Jun 4;(6):CD007867. http://dx.doi.org/10.1002/14651858.CD00786 7.pub2. PMID: 29864793.

29. Rasmussen NML, Belqaid K, Lugnet K, et al. Effectiveness of multidisciplinary nutritional support in older hospitalised patients: a systematic review and meta-analyses. Clin Nutr ESPEN. 2018 Oct;27:44-52. http://dx.doi.org/10.1016/j.clnesp.2018.07.002 . PMID: 30144892.

30. Re LG, Lusignani M. Effectiveness of oral nutritional supplements to prevent hospital malnutrition. Overview of systematic reviews. Assistenza infermieristica e ricerca: AIR. 2018 Jan;37(1):25-41. http://dx.doi.org/10.1702/2890.29149. PMID: 29658538.

31. Shi J, Wei L, Huang R, et al. Effect of combined parenteral and enteral nutrition versus enteral nutrition alone for critically ill patients: a systematic review and metaanalysis. Medicine (Baltimore). 2018 Oct;97(41):e11874. http://dx.doi.org/10.1097/MD.0000000000011 874. PMID: 30313021.

32. Wyers CE, Reijven PLM, Breedveld-Peters JJL, et al. Efficacy of nutritional intervention in elderly after hip fracture: a multicenter randomized controlled trial. J Gerontol A Biol Sci Med Sci. 2018 Sep 11;73(10):1429-37. http://dx.doi.org/10.1093/gerona/gly030. PMID: 30204859.

33. Cano-Torres EA, Simental-Mendía LE, Morales-Garza LA, et al. Impact of nutritional intervention on length of hospital stay and mortality among hospitalized patients with malnutrition: a clinical randomized controlled trial. J Am Coll Nutr. 2017 May 19;36(4):2359.

http://dx.doi.org/10.1080/07315724.2016.1259 595. PMID: 28318388.

34. Feinberg J, Nielsen EE, Korang SK, et al. Nutrition support in hospitalised adults at nutritional risk. Cochrane Database Syst Rev. 2017 May 19;(5):CD011598.

http://dx.doi.org/10.1002/14651858.CD01159 8.pub2. PMID: 28524930.
35. García-Rodríguez MT, López-Calviño B, Piñón-Villar MDC, et al. Concordance among methods of nutritional assessment in patients included on the waiting list for liver transplantation. J Epidemiol. 2017 Oct;27(10):469-75. http://dx.doi.org/10.1016/j.je.2016.09.011. PMID: 28549896.

36. Sriram K, Sulo S, Vanderbosch G, et al. A comprehensive nutrition-focused quality improvement program reduces 30-day readmissions and length of stay in hospitalized patients. J Parenter Enteral Nutr. 2017 Mar;41(3):384-91. http://dx.doi.org/10.1177/0148607116681468. PMID: 27923890.

37. Aust J, Werner A, Grünewald G, et al. Results of "screening for malnutrition" in a major community hospital. Aktuel Ernahrungsmed. 2016 Oct;41(5):352-8. http://dx.doi.org/10.1055/s-0042-116657.

38. Baldwin C, Kimber KL, Gibbs M, et al. Supportive interventions for enhancing dietary intake in malnourished or nutritionally at-risk adults. Cochrane Database Syst Rev. 2016 Dec 20;(12):CD009840. http://dx.doi.org/10.1002/14651858.CD00984 0.pub2. PMID: 27996085.

39. Bally MR, Yildirim PZB, Bounoure L, et al. Nutritional support and outcomes in malnourished medical inpatients a systematic review and meta-analysis. JAMA Intern Med. 2016 Jan;176(1):43-53.

http://dx.doi.org/10.1001/jamainternmed.2015. 6587. PMID: 26720894.

40. Õzbilgin S, Hanci V, Ömür D, et al. Morbidity and mortality predictivity of nutritional assessment tools in the postoperative care unit. Medicine (Baltimore). 2016 Oct;95(40):e5038. PMID: 27749567.

41. Calleja Fernández A, Pintor de la Maza B, Vidal Casariego A, et al. Food intake and nutritional status influence outcomes in hospitalized hematology-oncology patients. Nutr Hosp. 2015 Jun;31(6):2598-605. http://dx.doi.org/10.3305/nh.2015.31.6.8674. PMID: 26040371. 
42. Casals C, García-Agua-Soler N, VázquezSànchez MÃ, et al. Randomized clinical trial of nutritional counseling for malnourished hospital patients. Rev Clin Esp. 2015

Aug;215(6):308-14.

http://dx.doi.org/10.1016/j.rce.2015.02.012. PMID: 25816982.

43. da Silva Fink J, Daniel de Mello P, Daniel de Mello E. Subjective global assessment of nutritional status - a systematic review of the literature. Clin Nutr. 2015 Oct;34(5):785-92. http://dx.doi.org/10.1016/j.clnu.2014.12.014. PMID: 25596153.

44. Rossi RE, Conte D, Massironi S. Diagnosis and treatment of nutritional deficiencies in alcoholic liver disease: overview of available evidence and open issues. Dig Liver Dis. 2015 Oct;47(10):819-25. http://dx.doi.org/10.1016/j.dld.2015.05.021. PMID: 26164399.

45. Sun Z, Kong XJ, Jing X, et al. Nutritional risk screening 2002 as a predictor of postoperative outcomes in patients undergoing abdominal surgery: a systematic review and meta-analysis of prospective cohort studies. PLoS ONE. 2015 Jul 14;10(7):e0132857. http://dx.doi.org/10.1371/journal.pone.013285 7. PMID: 26172830.

46. Tapia MJ, Ocón J, Cabrejas-Gómez C, et al. Nutrition-related risk indexes and long-term mortality in noncritically ill inpatients who receive total parenteral nutrition (prospective multicenter study). Clin Nutr. 2015

Oct;34(5):962-7.

http://dx.doi.org/10.1016/j.clnu.2014.10.008. PMID: 25466952.

47. Agarwal E, Ferguson M, Banks M, et al. Malnutrition and poor food intake are associated with prolonged hospital stay, frequent readmissions, and greater in-hospital mortality: results from the Nutrition Care Day Survey 2010. Clin Nutr. 2013 Oct;32(5):73745.

http://dx.doi.org/10.1016/j.clnu.2012.11.021. PMID: 23260602.

48. Lomivorotov VV, Efremov SM, Boboshko $\mathrm{VA}$, et al. Evaluation of nutritional screening tools among patients scheduled for heart valve surgery. J Heart Valve Dis. 2013

Mar;22(2):239-47. PMID: 23798215.
49. Milte RK, Ratcliffe J, Miller MD, et al. Economic evaluation for protein and energy supplementation in adults: opportunities to strengthen the evidence. Eur J Clin Nutr. 2013 Dec;67(12):1243-50. http://dx.doi.org/10.1038/ejen.2013.206. PMID: 24169464.

50. Olveira G, Tapia MJ, Ocón J, et al. Study Group of Hyperglycemia in Parenteral Nutrition; Nutrition Area of the Spanish Society of Endocrinology and Nutrition (SEEN). The subjective global assessment predicts in-hospital mortality better than other nutrition-related risk indexes in noncritically ill inpatients who receive total parenteral nutrition in Spain (prospective multicenter study). J Acad Nutr Diet. 2013

Sep;113(9):1209-18. http://dx.doi.org/10.1016/j.jand.2013.05.027. PMID: 23972272.

51. Omidvari AH, Vali Y, Murray SM, et al. Nutritional screening for improving professional practice for patient outcomes in hospital and primary care settings. Cochrane Database Syst Rev. 2013 Jun 6;(6):CD005539. http://dx.doi.org/10.1002/14651858.CD00553 9.pub2. PMID: 23744516.

52. Collins PF, Stratton RJ, Elia M. Nutritional support in chronic obstructive pulmonary disease: a systematic review and metaanalysis. Am J Clin Nutr. 2012 Jun;95(6):1385-95.

53. Moon KT. Reducing post-hospital mortality via individualized nutrition plan. Am Fam Physician. 2012 Jan 15;85(2):200. https://www.aafp.org/afp/2012/0115/p200.htm $\underline{1 .}$

54. Neelemaat F, Lips P, Bosmans JE, et al. Shortterm oral nutritional intervention with protein and vitamin $\mathrm{D}$ decreases falls in malnourished older adults. J Am Geriatr Soc. 2012 Apr;60(4):691-9. http://dx.doi.org/10.1111/j.15325415.2011.03888.x. PMID: 22316322.

55. Klek S, Sierzega M, Szybinski $P$, et al. Perioperative nutrition in malnourished surgical cancer patients - a prospective, randomized, controlled clinical trial. Clin Nutr. 2011 Dec;30(6):708-13. http://dx.doi.org/10.1016/j.clnu.2011.07.007. PMID: 21820770. 
56. Klek S, Sierzega M, Szybinski P, et al. The immunomodulating enteral nutrition in malnourished surgical patients - a prospective, randomized, double-blind clinical trial. Clin Nutr. 2011 Jun;30(3):282-8. http://dx.doi.org/10.1016/j.clnu.2010.10.001. PMID: 21074910.

57. Velasco C, García E, Rodríguez V, et al. Comparison of four nutritional screening tools to detect nutritional risk in hospitalized patients: a multicentre study. Eur J Clin Nutr. 2011 Feb;65(2):269-74. http://dx.doi.org/10.1038/ejen.2010.243. PMID: 21081958.

58. Neelemaat F, Thijs A, Seidell JC, et al. Study protocol: cost-effectiveness of transmural nutritional support in malnourished elderly patients in comparison with usual care. Nutr J. 2010 Feb 10;9:6. http://dx.doi.org/10.1186/1475-2891-9-6. PMID: 20146794.

59. Cansado P, Ravasco P, Camilo M. A longitudinal study of hospital undernutrition in the elderly: comparison of four validated methods. J Nutr Health Aging. 2009 Feb;13(2):159-64. http://dx.doi.org/10.1007/s12603-009-0024-y. PMID: 19214346.

60. Milne AC, Potter J, Vivanti A, et al. Protein and energy supplementation in elderly people at risk from malnutrition. Cochrane Database Syst Rev. 2009 Apr 15;(2):CD003288. http://dx.doi.org/10.1002/14651858.CD00328 8.pub3. PMID: 19370584.

61. Baldwin C, Weekes CE. Dietary advice for illness-related malnutrition in adults. Cochrane Database Syst Rev. 2008 Jan 23;(1):CD002008.

62. Pronio A, Di Filippo A, Aguzzi D, et al. Treatment of mild malnutrition and reduction of morbidity in abdominal surgery: a trial on 153 patients. Clin Ter. 2008 JanFeb;159(1):13-8. PMID: 18399256.

63. Koretz RL, Avenell A, Lipman TO, et al. Does enteral nutrition affect clinical outcome? A systematic review of the randomized trials. Am J Gastroenterol. 2007 Feb;102(2):412-29; quiz 468.
64. Vanderkroft D, Collins CE, FitzGerald M, et al. Minimising undernutrition in the older inpatient. JBI Libr Syst Rev. 2007;5(3):1-96. http://dx.doi.org/10.11124/01938924200705030-00001. PMID: 27820021.

65. Martins CP, Correia JR, Do Amaral TF. Undernutrition risk screening and length of stay of hospitalized elderly. J Nutr Elder. 2006 Dec 13;25(2):5-21. http://dx.doi.org/10.1300/J052v25n02 02. PMID: 17182464.

66. de Luis D, Lopez Guzman A. Nutritional status of adult patients admitted to internal medicine departments in public hospitals in Castilla y Leon, Spain - a multi-center study. Eur J Intern Med. 2006 Dec;17(8):556-60. http://dx.doi.org/10.1016/j.ejim.2006.02.030. PMID: 17142174.

67. Miller MD, Crotty M, Whitehead C, et al. Nutritional supplementation and resistance training in nutritionally at risk older adults following lower limb fracture: a randomized controlled trial. Clin Rehabil. 2006 Apr;20(4):311-23. http://dx.doi.org/10.1191/0269215506cr942oa. PMID: 16719029.

68. Kruizenga HM, Van Tulder MW, Seidell JC, et al. Effectiveness and cost-effectiveness of early screening and treatment of malnourished patients. Am J Clin Nutr. 2005;82(5):1082-9. PMID: 16280442.

69. Braunschweig C, Liang H, Sheean P. Indications for administration of parenteral nutrition in adults. Nutr Clin Pract. 2004 Jun;19(3):255-62. http://dx.doi.org/10.1177/01154265040190032 55. PMID: 16215113 .

70. Hickson M, Bulpitt C, Nunes M, et al. Does additional feeding support provided by health care assistants improve nutritional status and outcome in acutely ill older in-patients? A randomised control trial. Clin Nutr. 2004 Feb;23(1):69-77. http://dx.doi.org/10.1016/S02615614(03)00090-6. PMID: 14757395.

71. Rypkema G, Adang E, Dicke H, et al. Costeffectiveness of an interdisciplinary intervention in geriatric inpatients to prevent malnutrition. J Nutr Health Aging. 2004;8(2):122-7. PMID: 14978608. 
72. Kondrup J, Ramussen HH, Hamberg O, et al. Nutritional risk screening (NRS 2002): a new method based on an analysis of controlled clinical trials. Clin Nutr. 2003 Jun;22(3):32136. http://dx.doi.org/10.1016/S02615614(02)00214-5. PMID: 12765673.

73. Potter JM, Roberts MA, McColl JH, et al. Protein energy supplements in unwell elderly patients - a randomized controlled trial. JPEN J Parenter Enteral Nutr. 2001;25(6):323-9. http://dx.doi.org/10.1177/01486071010250063 23. PMID: 11688936 .

74. Dijkink S, Meier K, Krijnen P, et al. Malnutrition and its effects in severely injured trauma patients. Eur J Trauma Emerg Surg. 2020 Oct;46(5):993-1004. Epub 2020 Jan 23. http://dx.doi.org/10.1007/s00068-020-013045. PMID: 31974669.

75. Ney M, Li S, Vandermeer B, et al. Systematic review with meta-analysis: nutritional screening and assessment tools in cirrhosis. Liver Int. 2020 Mar;40(3):664-73. http://dx.doi.org/10.1111/liv.14269. PMID: 31571398.

76. Muscaritoli M, Krznaric Z, Singer P, et al. Effectiveness and efficacy of nutritional therapy: a systematic review following Cochrane methodology. Clin Nutr. 2017 Aug;36(4):939-57. http://dx.doi.org/10.1016/j.clnu.2016.06.022. PMID: 27448948.

77. Lew CCH, Yandell R, Fraser RJL, et al. Association between malnutrition and clinical outcomes in the intensive care unit: a systematic review. J Parenter Enteral Nutr. 2017 Jul;41(5):744-58. http://dx.doi.org/10.1177/0148607115625638. PMID: 26838530.

78. Lin H, Zhang H, Lin Z, et al. Review of nutritional screening and assessment tools and clinical outcomes in heart failure. Heart Fail Rev. 2016 Sep;21(5):549-65. http://dx.doi.org/10.1007/s10741-016-9540-0. PMID: 26920682.

79. Gupta D, Vashi PG, Lammersfeld CA, et al. Role of nutritional status in predicting the length of stay in cancer: a systematic review of the epidemiological literature. Ann Nutr Metab. 2011 Dec;59(2):96-106. http://dx.doi.org/10.1159/000332914. PMID: 22142719.
80. Bonilla-Palomas JL, Gámez-López AL, Castillo-Domînguez JC, et al. Nutritional intervention in malnourished hospitalized patients with heart failure. Arch Med Res. 2016 Oct;47(7):535-40.

http://dx.doi.org/10.1016/j.arcmed.2016.11.00 5. PMID: 28262195.

81. Deutz NE, Matheson EM, Matarese LE, et al. Readmission and mortality in malnourished, older, hospitalized adults treated with a specialized oral nutritional supplement: a randomized clinical trial. Clin Nutr. 2016 Feb;35(1):18-26.

http://dx.doi.org/10.1016/j.clnu.2015.12.010. PMID: 26797412.

82. Gazzotti C, Arnaud-Battandier F, Parello M, et al. Prevention of malnutrition in older people during and after hospitalisation: results from a randomised controlled clinical trial. Age Ageing. 2003 May;32(3):321-5. http://dx.doi.org/10.1093/ageing/32.3.321. PMID: 12720620.

83. Ha L, Hauge T, Spenning AB, et al. Individual, nutritional support prevents undernutrition, increases muscle strength and improves QoL among elderly at nutritional risk hospitalized for acute stroke: a randomized, controlled trial. Clin Nutr. 2010 Oct;29(5):567-73.

http://dx.doi.org/10.1016/j.clnu.2010.01.011. PMID: 20176418.

84. Holyday M, Daniells S, Bare M, et al. Malnutrition screening and early nutrition intervention in hospitalised patients in acute aged care: a randomised controlled trial. J Nutr Health Aging. 2012 Jun;16(6):562-8. http://dx.doi.org/10.1007/s12603-012-0022-3. PMID: 22659998.

85. Johansen N, Kondrup J, Plum LM, et al. Effect of nutritional support on clinical outcome in patients at nutritional risk. Clin Nutr. 2004 Aug;23(4):539-50. PMID: 15297090.

86. Munk T, Beck AM, Holst M, et al. Positive effect of protein-supplemented hospital food on protein intake in patients at nutritional risk: a randomised controlled trial. J Hum Nutr Diet. 2014 Apr;27(2):122-32. http://dx.doi.org/10.1111/jhn.12210. PMID: 24479388. 
87. Rüfenacht U, Rühlin M, Wegmann M, et al. Nutritional counseling improves quality of life and nutrient intake in hospitalized undernourished patients. Nutrition. 2010 Jan;26(1):53-60. http://dx.doi.org/10.1016/j.nut.2009.04.018. PMID: 19695832.

88. Schuetz P, Fehr R, Baechli V, et al. Individualised nutritional support in medical inpatients at nutritional risk: a randomised clinical trial. Lancet. 2019 Jun 8;393(10188):2312-21. http://dx.doi.org/10.1016/S01406736(18)32776-4. PMID: 31030981.

89. Sharma Y, Thompson CH, Kaambwa B, et al. Investigation of the benefits of early malnutrition screening with telehealth follow up in elderly acute medical admissions. QJM. 2017 Oct;110(10):639-47. http://dx.doi.org/10.1093/qimed/hcx095. PMID: 28472530.

90. Starke J, Schneider H, Alteheld B, et al. Shortterm individual nutritional care as part of routine clinical setting improves outcome and quality of life in malnourished medical patients. Clin Nutr. 2011 Apr;30(2):194-201. http://dx.doi.org/10.1016/j.clnu.2010.07.021. PMID: 20937544.

91. Acehan S, Gulen M, Satar S, et al. Evaluation of nutrition risk in patients over 65 years of age with nontraumatic acute abdominal syndrome. Nutr Clin Pract. 2020 Dec;35(6):1070-9. http://dx.doi.org/10.1002/ncp.10575. PMID: 32935880 .

92. Bedock D, Bel Lassen P, Mathian A, et al. Prevalence and severity of malnutrition in hospitalized COVID-19 patients. Clin Nutr ESPEN. 2020 Dec;40:214-9. http://dx.doi.org/10.1016/j.clnesp.2020.09.018 . PMID: 33183539.

93. Brascher JMM, Peres WAF, Padilha PC. Use of the modified "nutrition risk in the critically ill" score and its association with the death of critically ill patients. Clin Nutr ESPEN. 2020 Feb;35:162-6. http://dx.doi.org/10.1016/j.clnesp.2019.10.005 . PMID: 31987111.
94. Burgos R, Joaquín C, Blay C, et al. Diseaserelated malnutrition in hospitalized chronic patients with complex needs. Clin Nutr. 2020 May;39(5):1447-53.

http://dx.doi.org/10.1016/j.clnu.2019.06.006. PMID: 31256806.

95. Dou L, Wang X, Cao Y, et al. Relationship between postoperative recovery and nutrition risk screened by NRS 2002 and nutrition support status in patients with gastrointestinal cancer. Nutr Cancer. 2020 Jan;72(1):33-40. http://dx.doi.org/10.1080/01635581.2019.1612 927. PMID: 31079488.

96. Galindo Martín CA, Aportela Vázquez VA, Becerril Hernández F, et al. The GLIM criteria for adult malnutrition and its relation with adverse outcomes, a prospective observational study. Clin Nutr ESPEN. 2020 Aug;38:67-73. http://dx.doi.org/10.1016/j.clnesp.2020.06.015 . PMID: 32690180.

97. Han TS, Yeong K, Lisk R, et al. Prevalence and consequences of malnutrition and malnourishment in older individuals admitted to hospital with a hip fracture. Eur J Clin Nutr. 2020 Oct 7; Online ahead of print. http://dx.doi.org/10.1038/s41430-020-007745. PMID: 33028971.

98. Hirose S, Miyazaki S, Yatsu S, et al. Impact of the geriatric nutritional risk index on inhospital mortality and length of hospitalization in patients with acute decompensated heart failure with preserved or reduced ejection fraction. J Clin Med. 2020 Apr;9(4):1169. http://dx.doi.org/10.3390/jcm9041169.

99. Kaddoura R, Shanks A, Chapman M, et al. Relationship between nutritional status on admission to the intensive care unit and clinical outcomes. Nutr Diet. 2020 Sep 28; Online ahead of print. http://dx.doi.org/10.1111/1747-0080.12637. PMID: 32985110.

100. Karim AM, Aljammal M, Panhwar MS, et al. Impact of malnutrition and interventions on patients receiving heart transplant. J Heart Lung Transplant. 2020 Apr;39(4):S256-S257. http://dx.doi.org/10.1016/j.healun.2020.01.556 
101. Karin M, Bogut A, Hojsak I, et al. Nutritional status and its effect on complications in patients with colorectal cancer. Wien Klin Wochenschr. 2020 Aug;132(15):431-7. http://dx.doi.org/10.1007/s00508-020-016714. PMID: 32451819.

102. Kootaka Y, Kamiya K, Hamazaki N, et al. The GLIM criteria for defining malnutrition can predict physical function and prognosis in patients with cardiovascular disease. Clin Nutr. 2021 Jan;40(1):146-52. http://dx.doi.org/10.1016/j.clnu.2020.04.038. PMID: 32571679.

103. Machado dos Reis A, Marchetti J, Forte dos Santos A, et al. NUTRIC Score: isolated and combined use with the NRS-2002 to predict hospital mortality in critically ill patients. $\mathrm{J}$ Parenter Enteral Nutr. 2020 Sep;44(7):1250-6. http://dx.doi.org/10.1002/jpen.1804. PMID: 32026516.

104. Matsumoto Y, Iwai K, Namikawa N, et al. The relationship between existing nutritional indicators and Global Leadership Initiative on Malnutrition (GLIM) criteria: a one-institution cross-sectional analysis. Clin Nutr. 2020 Oct;39(10):3099-104. http://dx.doi.org/10.1016/j.clnu.2020.01.016. PMID: 32046880.

105. Maurer E, Wallmeier V, Reumann MK, et al. Risk of malnutrition in orthopedic trauma patients with surgical site infections is associated with increased morbidity and mortality - a 3-year follow-up study. Injury. 2020 Oct;51(10):2219-29.

http://dx.doi.org/10.1016/j.injury.2020.06.019. PMID: 32620329.

106. Pratt KJ, Hernandez B, Blancato R, et al. Impact of an interdisciplinary malnutrition quality improvement project at a large metropolitan hospital. BMJ Open Qual. 2020 Mar;9(1):e000735.

http://dx.doi.org/10.1136/bmjoq-2019-000735. PMID: 32213547.

107. Tao J, Ke YY, Zhang Z, et al. Comparison of the value of malnutrition and sarcopenia for predicting mortality in hospitalized old adults over 80 years. Exp Gerontol. 2020

Sep;138:111007. http://dx.doi.org/10.1016/j.exger.2020.111007. PMID: 32590128.
108. Toledo DO, Silva Junior JM, Toloi JM, et al. NUTRIC-S proposal: using SAPS 3 for mortality prediction in nutritional risk ICU patients. Clinical Nutrition Experimental. 2020 Jun;31:19-27. http://dx.doi.org/10.1016/j.yclnex.2019.12.003

109. Tonet E, Campo G, Maietti E, et al. Nutritional status and all-cause mortality in older adults with acute coronary syndrome. Clin Nutr. 2020 May;39(5):1572-9. http://dx.doi.org/10.1016/j.clnu.2019.06.025. PMID: 31324416.

110. Trestini I, Sperduti I, Sposito M, et al. Evaluation of nutritional status in non-smallcell lung cancer: screening, assessment and correlation with treatment outcome. ESMO Open. 2020 May 17;5(3):e000689. http://dx.doi.org/10.1136/esmoopen-2020000689. PMID: 32424067.

111. Yilmaz M, Atilla FD, Sahin F, et al. The effect of malnutrition on mortality in hospitalized patients with hematologic malignancy.

Support Care Cancer. 2020 Mar;28(3):1441-8. http://dx.doi.org/10.1007/s00520-019-049525. PMID: 31273503.

112. Zhao Y, Xia X, Xie D, et al. Geriatric Nutritional Risk Index can predict postoperative delirium and hospital length of stay in elderly patients undergoing non-cardiac surgery. Geriatr Gerontol Int. 2020 Aug;20(8):759-64. http://dx.doi.org/10.1111/ggi.13963. PMID: 32570290 .

113. Nishi I, Seo Y, Hamada-Harimura Y, et al. Geriatric nutritional risk index predicts allcause deaths in heart failure with preserved ejection fraction. ESC Heart Fail. 2019 Apr;6(2):396-405. http://dx.doi.org/10.1002/ehf2.12405. PMID: 30706996.

114. Abd-Elraheem M, Mashav N, Ioffe M, et al. Which laboratory malnutrition markers best predict 1-year mortality in hospitalized older adults? Eur Geriatr Med. 2019 Aug;10(4):61924. http://dx.doi.org/10.1007/s41999-01900204-1. 
115. Almasaudi AS, McSorley ST, Dolan RD, et al. The relation between Malnutrition Universal Screening Tool (MUST), computed tomography-derived body composition, systemic inflammation, and clinical outcomes in patients undergoing surgery for colorectal cancer. Am J Clin Nutr. 2019

Dec;110(6):1327-34. http://dx.doi.org/10.1093/ajen/nqz230. PMID: 31529042.

116. Chien SC, Lo CI, Lin CF, et al. Malnutrition in acute heart failure with preserved ejection fraction: clinical correlates and prognostic implications. ESC Heart Fail. 2019 Oct;6(5):953-64. http://dx.doi.org/10.1002/ehf2.12501. PMID: 31400092.

117. Contreras-Bolívar V, Sánchez-Torralvo FJ, Ruiz-Vico M, et al. GLIM criteria using hand grip strength adequately predict six-month mortality in cancer inpatients. Nutrients. 2019 Sep;11(9):2043.

http://dx.doi.org/10.3390/nu11092043. PMID: 31480635 .

118. Gonzalez MC, Bielemann RM, Kruschardt PP, et al. Complementarity of NUTRIC score and subjective global assessment for predicting 28day mortality in critically ill patients. Clin Nutr. 2019 Dec;38(6):2846-50. http://dx.doi.org/10.1016/j.clnu.2018.12.017. PMID: 30595375.

119. Gottschall C, Tarnowski M, Machado P, et al. Predictive and concurrent validity of the malnutrition universal screening tool using mid-upper arm circumference instead of body mass index. J Hum Nutr Diet. 2019 Dec;32(6):775-80. http://dx.doi.org/10.1111/jhn.12665. PMID: 31067603.

120. Hirose EY, de Molla VC, Gonçalves MV, et al. The impact of pretransplant malnutrition on allogeneic hematopoietic stem cell transplantation outcomes. Clin Nutr ESPEN. 2019 Oct;33:213-9. http://dx.doi.org/10.1016/j.clnesp.2019.05.005 . PMID: 31451264.
121. Inoue $\mathrm{T}, \mathrm{Misu} \mathrm{S}$, Tanaka $\mathrm{T}$, et al. Acute phase nutritional screening tool associated with functional outcomes of hip fracture patients: a longitudinal study to compare MNA-SF, MUST, NRS-2002 and GNRI. Clin Nutr. 2019 Feb;38(1):220-6. http://dx.doi.org/10.1016/j.clnu.2018.01.030. PMID: 29456030.

122. Komici K, Vitale DF, Mancini A, et al. Impact of malnutrition on long-term mortality in elderly patients with acute myocardial infarction. Nutrients. 2019 Jan 22;11(2):224. http://dx.doi.org/10.3390/nu11020224. PMID: 30678180 .

123. Minamisawa M, Seidelmann SB, Claggett B, et al. Impact of malnutrition using geriatric nutritional risk index in heart failure with preserved ejection fraction. JACC Heart Fail. 2019 Aug;7(8):664-75. http://dx.doi.org/10.1016/j.jchf.2019.04.020. PMID: 31302049.

124. Müller M, Dahdal S, Saffarini M, et al. Evaluation of nutrition risk screening score 2002 (NRS) assessment in hospitalized chronic kidney disease patient. PLoS ONE. 2019 Jan 24;14(1):e0211200. http://dx.doi.org/10.1371/journal.pone.021120 0. PMID: 30677081 .

125. Ruiz AJ, Buitrago G, Rodríguez N, et al. Clinical and economic outcomes associated with malnutrition in hospitalized patients. Clin Nutr. 2019 Jun;38(3):1310-6. http://dx.doi.org/10.1016/j.clnu.2018.05.016. PMID: 29891224.

126. Sauer AC, Goates S, Malone A, et al. Prevalence of malnutrition risk and the impact of nutrition risk on hospital outcomes: results from nutrition day in the U.S. J Parenter Enteral Nutr. 2019 Sep;43(7):918-26. http://dx.doi.org/10.1002/jpen.1499. PMID: 30666659 .

127. Subwongcharoen $\mathrm{S}$, Areesawangvong $\mathrm{P}$, Chompoosaeng T. Impact of nutritional status on surgical patients. Clin Nutr ESPEN. 2019 Aug;32:135-9. http://dx.doi.org/10.1016/j.clnesp.2019.03.016 . PMID: 31221278. 
128. Viana MV, Pantet O, Bagnoud G, et al. Metabolic and nutritional characteristics of long-stay critically ill patients. J Clin Med. 2019 Jul 7;8(7):985.

http://dx.doi.org/10.3390/jem8070985. PMID: 31284633.

129. Ceniccola GD, Holanda TP, Pequeno RSF, et al. Relevance of AND-ASPEN criteria of malnutrition to predict hospital mortality in critically ill patients: a prospective study. J Crit Care. 2018 Apr;44:398-403. http://dx.doi.org/10.1016/j.jcrc.2017.12.013. PMID: 29306205.

130. Chen WZ, Chen XY, Zhang FM, et al. Effect of nutritional risk on short-term outcomes after laparoscopic-assisted gastrectomy: a prospective study from two centers. Eur J Gastroenterol Hepatol. 2018;30(2):136-42. http://dx.doi.org/10.1097/MEG.000000000000 1017. PMID: 29227327.

131. Dent E, Wright O, Hoogendijk EO, et al. Nutritional screening and dietitian consultation rates in a geriatric evaluation and management unit. Nutr Diet. 2018 Feb;75(1):11-6. http://dx.doi.org/10.1111/1747-0080.12391. PMID: 29164776.

132. Marcadenti A, Mendes LL, Rabito EI, et al. Nutritional risk in emergency-2017: a new simplified proposal for a nutrition screening tool. J Parenter Enteral Nutr. 2018

Sep;42(7):1168-76. http://dx.doi.org/10.1002/jpen.1147. PMID: 29534291.

133. Morris N, Stewart S, Riley M, et al. Differential impact of malnutrition on health outcomes among indigenous and nonindigenous adults admitted to hospital in regional Australia -- a prospective cohort study. Nutrients. 2018 May 19;10(5):644. http://dx.doi.org/10.3390/nu10050644. PMID: 29783749.

134. Ramos R, Nadal E, Peiró I, et al. Preoperative nutritional status assessment predicts postoperative outcomes in patients with surgically resected non-small cell lung cancer. Eur J Surg Oncol. 2018 Sep;44(9):1419-24. http://dx.doi.org/10.1016/j.ejso.2018.03.026. PMID: 29685760.
135. Rondel ALMA, Langius JAE, de van der Schueren MAE, et al. The new ESPEN diagnostic criteria for malnutrition predict overall survival in hospitalised patients. Clin Nutr. 2018 Feb;37(1):163-8. http://dx.doi.org/10.1016/j.clnu.2016.11.018. PMID: 27939358.

136. Borek P, Chmielewski M, Magorzewicz S, et al. Analysis of outcomes of the NRS 2002 in patients hospitalized in nephrology wards. Nutrients. 2017 Mar 16;9(3):287. http://dx.doi.org/10.3390/nu9030287. PMID: 28300757.

137. Guerra RS, Fonseca I, Sousa AS, et al. ESPEN diagnostic criteria for malnutrition -- a validation study in hospitalized patients. Clin Nutr. 2017 Oct;36(5):1326-32.

http://dx.doi.org/10.1016/j.clnu.2016.08.022. PMID: 27642055.

138. Inoue T, Misu S, Tanaka T, et al. Pre-fracture nutritional status is predictive of functional status at discharge during the acute phase with hip fracture patients: a multicenter prospective cohort study. Clin Nutr. 2017 Oct;36(5):13205. http://dx.doi.org/10.1016/j.clnu.2016.08.021. PMID: 27612921.

139. Kalaiselvan MS, Renuka MK, Arunkumar AS. Use of nutrition risk in critically ill (NUTRIC) score to assess nutritional risk in mechanically ventilated patients: a prospective observational study. Indian J Crit Care Med. 2017 May;21(5):253-6. http://dx.doi.org/10.4103/ijccm.IJCCM-24-17. PMID: 28584426.

140. Kirushnan BB, Subba Rao B, Annigeri R, et al. Impact of malnutrition, inflammation, and atherosclerosis on the outcome in hemodialysis patients. Indian J Nephrol. 2017 Jul;27(4):277-83. http://dx.doi.org/10.4103/0971-4065.202830. PMID: 28761229.

141. Leiva Badosa E, Badia Tahull M, Virgili Casas N, et al. Hospital malnutrition screening at admission: malnutrition increases mortality and length of stay. Nutr Hosp. $2017 \mathrm{Jul}$ 28;34(4):907-13. http://dx.doi.org/10.20960/nh.657. PMID: 29095016. 
142. Martucci RB, Barbosa MV, D'Almeida CA, et al. Undernutrition as independent predictor of early mortality in elderly cancer patients.

Nutrition. 2017 Feb;34:65-70.

http://dx.doi.org/10.1016/j.nut.2016.09.011. PMID: 28063514.

143. Pierik VD, Meskers CGM, Van Ancum JM, et al. High risk of malnutrition is associated with low muscle mass in older hospitalized patients - a prospective cohort study. BMC Geriatr. 2017 Jun 5;17(1):118. http://dx.doi.org/10.1186/s12877-017-0505-5. PMID: 28583070.

144. Potyraa P, Ogonowska Z, Toboa A, et al. Utility of nutritional evaluation for the clinical risk assessment of postoperative complications. Does oncology need the NRS 2002 scale? Nowotwory. 2017 Dec 12;67(3):174-9. http://dx.doi.org/10.5603/NJO.2017.0029.

145. Rabito EI, Marcadenti A, Da Silva Fink J, et al. Nutritional Risk Screening 2002, Short Nutritional Assessment Questionnaire, Malnutrition Screening Tool, and Malnutrition Universal Screening Tool are good predictors of nutrition risk in an emergency service. Nutr Clin Pract. 2017 Aug;32(4):526-32. http://dx.doi.org/10.1177/0884533617692527. PMID: 28199797.

146. Salomon du Mont L, Leclerc B, Morgant MC, et al. Impact of nutritional state on critical limb ischemia early outcomes (DENUCRITICC Study). Ann Vasc Surg. 2017 Nov;45:10-5. http://dx.doi.org/10.1016/j.avsg.2017.04.030. PMID: 28495537.

147. Sharma Y, Thompson C, Shahi R, et al. Malnutrition in acutely unwell hospitalized elderly - "The skeletons are still rattling in the hospital closet". J Nutr Health Aging. 2017 Dec;21(10):1210-5. http://dx.doi.org/10.1007/s12603-017-0903-6. PMID: 29188881.

148. Söderström L, Rosenblad A, Thors Adolfsson $\mathrm{E}$, et al. Malnutrition is associated with increased mortality in older adults regardless of the cause of death. Br J Nutr. 28 Feb 2017;117(4):532-40. http://dx.doi.org/10.1017/S0007114517000435 . PMID: 28290264.
149. Yun T, Ko YE, Kim SJ, et al. The additional benefit of weighted subjective global assessment (SGA) for the predictability of mortality in incident peritoneal dialysis patients: a prospective study. Medicine (Baltimore). 2017 Nov;96(44):e8421. http://dx.doi.org/10.1097/MD.0000000000008 421. PMID: 29095278.

150. Allard JP, Keller H, Jeejeebhoy KN, et al. Malnutrition at hospital admissioncontributors and effect on length of stay: a prospective cohort study from the Canadian Malnutrition Task Force. JPEN J Parenter Enteral Nutr. 2016 May;40(4):487-97. http://dx.doi.org/10.1177/0148607114567902. PMID: 25623481.

151. Bakshi N, Singh K. Nutrition assessment and its effect on various clinical variables among patients undergoing liver transplant Hepatobiliary Surg Nutr. 2016 Aug;5(4):35871.

http://dx.doi.org/10.21037/hbsn.2016.03.09. PMID: 27500148.

152. Gau BR, Chen HY, Hung SY, et al. The impact of nutritional status on treatment outcomes of patients with limb-threatening diabetic foot ulcers. J Diabetes Complications. 2016 Jan-Feb;30(1):138-42. http://dx.doi.org/10.1016/j.jdiacomp.2015.09.0 11. PMID: 26490754.

153. Guerra RS, Sousa AS, Fonseca I, et al. Comparative analysis of undernutrition screening and diagnostic tools as predictors of hospitalisation costs. J Hum Nutr Diet. 2016 Apr;29(2):165-73. http://dx.doi.org/10.1111/jhn.12288. PMID: 25532556.

154. Gultekin A, Memis D, Inal MT, et al. The impact of nutritional risk screening 2002 and subjective global assessment upon prognosis for intensive care patients. Prog Nutr. 2016;18(1):47-55. https://www.researchgate.net/publication/3015 84617 The impact of nutritional risk screen ing 2002 and subjective global assessment upon_prognosis for_intensive care patients. 
155. Koren-Hakim T, Weiss A, Hershkovitz A, et al. Comparing the adequacy of the MNA-SF, NRS-2002 and MUST nutritional tools in assessing malnutrition in hip fracture operated elderly patients. Clin Nutr. 2016

Oct;35(5):1053-8. http://dx.doi.org/10.1016/j.clnu.2015.07.014. PMID: 26231340.

156. Kruizenga H, Van Keeken S, Weijs P, et al. Undernutrition screening survey in 564,063 patients: patients with a positive undernutrition screening score stay in hospital $1.4 \mathrm{~d}$ longer. Am J Clin Nutr. 2016 Apr;103(4):1026-32. http://dx.doi.org/10.3945/ajcn.115.126615. PMID: 26961930.

157. Maasberg S, Knappe-Drzikova B, Vonderbeck $\mathrm{D}$, et al. Malnutrition predicts clinical outcome in patients with neuroendocrine neoplasia. Neuroendocrinology. 2016 Nov;104(1):11-25. http://dx.doi.org/10.1159/000442983. PMID: 26641457.

158. Sanz-París A, Gómez-Candela C, MartínPalmero Ã, et al. Application of the new ESPEN definition of malnutrition in geriatric diabetic patients during hospitalization: a multicentric study. Clin Nutr. 2016 Dec;35(6):1564-7. http://dx.doi.org/10.1016/j.clnu.2016.02.018. PMID: 26997334.

159. Tan SK, Loh YH, Choong HL, et al. Subjective global assessment for nutritional assessment of hospitalized patients requiring haemodialysis: a prospective cohort study. Nephrology (Carlton). 2016 Nov;21(11):9449. http://dx.doi.org/10.1111/nep.12707. PMID: 26715243.

160. van Wissen J, van Stijn MFM, Doodeman HJ, et al. Mini nutritional assessment and mortality after hip fracture surgery in the elderly. J Nutr Health Aging. 2016 Nov;20(9):964-8. http://dx.doi.org/10.1007/s12603-015-0630-9. PMID: 27791228.

161. Coltman A, Peterson S, Roehl K, et al. Use of 3 tools to assess nutrition risk in the intensive care unit. J Parenter Enteral Nutr. 2015 Jan 17;39(1):28-33. http://dx.doi.org/10.1177/0148607114532135. PMID: 24748598.
162. Cui J, Wan Q, Wu X, et al. Nutritional risk screening 2002 as a predictor of outcome during general ward-based noninvasive ventilation in chronic obstructive pulmonary disease with respiratory failure. Med Sci Monit. 2015 Sep 18;21:2786-93. http://dx.doi.org/10.12659/MSM.894191. PMID: 26386778.

163. Dent E, Chapman I, Piantadosi C, et al. Nutritional screening tools and anthropometric measures associate with hospital discharge outcomes in older people. Australas J Ageing. 2015 Mar;34(1):E1-6. http://dx.doi.org/10.1111/ajag.12130. PMID: 24444126

164. Felder S, Lechtenboehmer C, Bally M, et al. Association of nutritional risk and adverse medical outcomes across different medical inpatient populations. Nutrition. 2015 Nov;31(11):1385-93. http://dx.doi.org/10.1016/j.nut.2015.06.007. PMID: 26429660.

165. Grass F, Hübner M, Schäfer M, et al. Preoperative nutritional screening by the specialist instead of the nutritional risk score might prevent excess nutrition: a multivariate analysis of nutritional risk factors. Nutr J. 2015 Apr 16;14:37. http://dx.doi.org/10.1186/s12937-015-0024-1. PMID: 25890322.

166. Guerra RS, Fonseca I, Pichel F, et al. Usefulness of six diagnostic and screening measures for undernutrition in predicting length of hospital stay: a comparative analysis. J Acad Nutr Diet. 2015 Jun;115(6):927-38. http://dx.doi.org/10.1016/j.jand.2014.11.015. PMID: 25634094.

167. Jeejeebhoy KN, Keller H, Gramlich L, et al. Nutritional assessment: comparison of clinical assessment and objective variables for the prediction of length of hospital stay and readmission. Am J Clin Nutr. 2015 May;101(5):956-65. http://dx.doi.org/10.3945/ajen.114.098665. PMID: 25739926.

168. Leandro-Merhi VA, Braga de Aquino JL. Comparison of nutritional diagnosis methods and prediction of clinical outcomes in patients with neoplasms and digestive tract diseases. Clin Nutr. 2015 Aug;34(4):647-51. http://dx.doi.org/10.1016/j.clnu.2014.07.001. PMID: 25086473. 
169. Rodrigues CS, Lacerda MS, Chaves GV. Patient generated subjective global assessment as a prognosis tool in women with gynecologic cancer. Nutrition. 2015 Nov;31(11):1372-8. http://dx.doi.org/10.1016/j.nut.2015.06.001. PMID: 26429658.

170. Simpson F, Doig GS. Physical assessment and anthropometric measures for use in clinical research conducted in critically ill patient populations: an analytic observational study. $\mathrm{J}$ Parenter Enteral Nutr. 2015 Mar 15;39(3):31321. http://dx.doi.org/10.1177/0148607113515526. PMID: 24335005.

171. Sohrabi Z, Eftekhari MH, Eskandari MH, et al. Malnutrition-inflammation score and quality of life in hemodialysis patients: is there any correlation? Nephrourol Mon. 2015 May 23;7(3):e27445. http://dx.doi.org/10.5812/numonthly.7(3)2015. 27445. PMID: 26034747.

172. Bell JJ, Bauer JD, Capra S, et al. Concurrent and predictive evaluation of malnutrition diagnostic measures in hip fracture inpatients: a diagnostic accuracy study. Eur J Clin Nutr. 2014 Mar;68(3):358-62. http://dx.doi.org/10.1038/ejen.2013.276. PMID: 24398643.

173. Chermesh I, Hajos J, Mashiach T, et al. Malnutrition in cardiac surgery: food for thought. Eur J Prev Cardiol. 2014 Apr;21(4):475-83. http://dx.doi.org/10.1177/2047487312452969. PMID: 22739686.

174. Cui H, Li Z, Zhu J. Correlation of the nutritional status with liver function and clinical outcomes in surgically treated liver cancer patients. Chinese Journal of Clinical Nutrition. 2014 Apr;22(2):82-6. http://dx.doi.org/10.3760/cma.j.issn.1674635X.2014.02.003.

175. de Mendonça Soares BL, Pessoa de Araújo Burgos MG. Nutritional risk among surgery patients and associations with hospital stay and postoperative complications. Nutr Hosp. 2014;30(3):636-42. http://dx.doi.org/10.3305/nh.2014.30.3.7633. PMID: 25238842.
176. Huang TH, Chi CC, Liu CH, et al. Nutritional status assessed by scored patient-generated subjective global assessment associated with length of hospital stay in adult patients receiving an appendectomy. Biomed J. 2014 Mar-Apr;37(2):71-7. http://dx.doi.org/10.4103/2319-4170.113183. PMID: 24732661.

177. Mendes J, Alves P, Amaral TF. Comparison of nutritional status assessment parameters in predicting length of hospital stay in cancer patients. Clin Nutr. 2014 Jun;33(3):466-70. http://dx.doi.org/10.1016/j.clnu.2013.06.016. PMID: 23849810.

178. Schrader E, Baumgartel C, Gueldenzoph H, et al. Nutritional status according to mini nutritional assessment is related to functional status in geriatric patients - Independent of health status. J Nutr Health Aging. 2014 Mar;18(3):257-63. http://dx.doi.org/10.1007/s12603-013-0394-z. PMID: 24626752.

179. Söderström L, Rosenblad A, Adolfsson ET, et al. Nutritional status predicts preterm death in older people: a prospective cohort study. Clin Nutr. 2014 Apr;33(2):354-9. http://dx.doi.org/10.1016/j.clnu.2013.06.004. PMID: 23810625.

180. Almeida AI, Correia M, Camilo M, et al. Length of stay in surgical patients: nutritional predictive parameters revisited. Br J Nutr. 2013 Jan 28;109(2):322-8. http://dx.doi.org/10.1017/S0007114512001134 . PMID: 22717003.

181. Holst M, Yifter-Lindgren E, Surowiak M, et al. Nutritional screening and risk factors in elderly hospitalized patients: association to clinical outcome? Scand J Caring Sci. 2013 Dec;27(4):953-61. http://dx.doi.org/10.1111/scs.12010. PMID: 23181396.

182. Lee JS, Choi HS, Ko YG, et al. Performance of the geriatric nutritional risk index in predicting 28-day hospital mortality in older adult patients with sepsis. Clin Nutr. 2013 Oct;32(5):843-8. http://dx.doi.org/10.1016/j.clnu.2013.01.007. PMID: 23391456. 
183. Rasheed S, Woods RT. Malnutrition and associated clinical outcomes in hospitalized patients aged 60 and older: an observational study in rural Wales. J Nutr Gerontol Geriatr. 2013 Jan;32(1):71-80. http://dx.doi.org/10.1080/21551197.2012.7537 72. PMID: 23451847.

184. Ulltang M, Vivanti AP, Murray E. Malnutrition prevalence in a medical assessment and planning unit and its association with hospital readmission. Aust Health Rev. 2013;37(5):636-41. http://dx.doi.org/10.1071/AH13051. PMID: 24200115.

185. Zhang SS, Tang ZY, Fang P, et al. Nutritional status deteriorates as the severity of diabetic foot ulcers increases and independently associates with prognosis. Exp Ther Med. 2013 Jan;5(1):215-22. http://dx.doi.org/10.3892/etm.2012.780.

186. Charlton K, Nichols C, Bowden S, et al. Poor nutritional status of older subacute patients predicts clinical outcomes and mortality at 18 months of follow-up. Eur J Clin Nutr. 2012 Nov;66(11):1224-8. http://dx.doi.org/10.1038/ejen.2012.130. PMID: 22990853.

187. Gamaletsou MN, Poulia KA, Karageorgou D, et al. Nutritional risk as predictor for healthcare-associated infection among hospitalized elderly patients in the acute care setting. J Hosp Infect. 2012 Feb;80(2):168-72. http://dx.doi.org/10.1016/j.jhin.2011.08.020. PMID: 22153954.

188. Komlanvi K, Jolly D, Vanaecke C, et al. Ability to predict six-month mortality and inter-tool agreement between four nutritional assessment instruments in the SAFES cohort. Eur Geriatr Med. 2012 Oct;3(5):285-9. http://dx.doi.org/10.1016/j.eurger.2012.06.002

189. Pavic T, Ljubicic N, Stojsavljevic S, et al. Nutritional screening model in tertiary medical unit in Croatia. Ann Nutr Metab. 2012 Aug;61(1):65-9. http://dx.doi.org/10.1159/000338967. PMID: 22831958.

190. Teiusanu A, Andrei M, Arbanas T, et al. Nutritional status in cirrhotic patients. Maedica (Buchar). 2012 Dec;7(4):284-9. PMID: 23483873.
191. Chermesh I, Ben-Ishay O, Gertsenzon H, et al. Malnutrition in surgical wards: a plea for concern. Geront Res Prac. 2011;2011:840512. http://dx.doi.org/10.1155/2011/840512. PMID: 20811544.

192. Karl A, Staehler M, Bauer R, et al. Malnutrition and clinical outcome in urological patients. Eur J Med Res. 2011 Dec 14;16(10):469-72. http://dx.doi.org/10.1186/2047-783X-16-10469. PMID: 22024426.

193. Köseoglu Z, Ozdogan M, Kuvvetli A, et al. Increased nutritional risk in major trauma: correlation with complications and prolonged length of stay. Ulus Travma Acil Cerrahi Derg. 2011;17(6):521-4. http://dx.doi.org/10.5505/tjtes.2011.28582. PMID: 22290005.

194. Hafsteinsdóttir TB, Mosselman M, Schoneveld $\mathrm{C}$, et al. Malnutrition in hospitalised neurological patients approximately doubles in 10 days of hospitalisation. J Clin Nurs. 2010 Mar;19(5):639-48. http://dx.doi.org/10.1111/j.13652702.2009.03142.x. PMID: 20500305.

195. Merli M, Giusto M, Gentili F, et al. Nutritional status: its influence on the outcome of patients undergoing liver transplantation. Liver Int. 2010 Feb;30(2):208-14. http://dx.doi.org/10.1111/j.14783231.2009.02135.x. PMID: 19840246.

196. Zamora RJ, Chavin H, Regazzoni CJ, et al. Nutritional status, systemic inflammatory response and mortality in the elderly hospitalized patient. Medicina (B Aires). 2010 May-Jun;70(3):233-9. PMID: 20529772.

197. Fiedler R, Jehle PM, Osten B, et al. Clinical nutrition scores are superior for the prognosis of haemodialysis patients compared to lab markers and bioelectrical impedance. Nephrol Dial Transplant. 2009 Dec;24(12):3812-7. http://dx.doi.org/10.1093/ndt/gfp346. PMID: 19605600.

198. Oliveira MR, Fogaça KC, Leandro-Merhi VA. Nutritional status and functional capacity of hospitalized elderly. Nutr J. 2009 Nov 17;8:54. http://dx.doi.org/10.1186/1475-2891-8-54. PMID: 19919711. 
199. Ozkalkanli MY, Ozkalkanli DT, Katircioglu $\mathrm{K}$, et al. Comparison of tools for nutrition assessment and screening for predicting the development of complications in orthopedic surgery. Nutr Clin Pract. 2009 Apr;24(2):27480.

http://dx.doi.org/10.1177/0884533609332087. PMID: 19321901.

200. Bin J, Jiang ZM, Zhu SN, et al. Methodology of research in influences of nutritional intervention and nutritional risk on clinical outcome. Chinese Journal of Clinical Nutrition. 2008 Dec;16(6):367-70. http://dx.doi.org/10.3881/j.issn.1008$\underline{5882.2008 .06 .010 .}$.

201. Casariego AV, Fernández MJI. Risk factors of malnutrition at hospital admission. Endocrinol Nutr. 2008 Jun;55(6):259-62. http://dx.doi.org/10.1016/S15750922(08)70680-6.

202. Henderson S, Moore N, Lee E, et al. Do the malnutrition universal screening tool (MUST) and Birmingham nutrition risk (BNR) score predict mortality in older hospitalised patients? BMC Geriatr. 2008 Oct 10;8:26. http://dx.doi.org/10.1186/1471-2318-8-26. PMID: 18847458.

203. Küçükardali Y, Yazgan Y, Solmazgül E, et al. Malnutrition screening with the nutritional risk screening 2002 in internal medicine service and the intensive care unit. Anatolian J Clin Invest. 2008;2(1):19-24.

204. Sungurtekin H, Sungurtekin U, Oner O, et al. Nutrition assessment in critically ill patients. Nutr Clin Pract. 2008 Dec-2009 Jan;23(6):635-41. PMID: 19033223.

205. Gurreebun F, Hartley GH, Brown AL, et al. Nutritional screening in patients on hemodialysis: is subjective global assessment an appropriate tool? J Ren Nutr. 2007 Mar;17(2):114-7. http://dx.doi.org/10.1053/j.jrn.2006.12.008. PMID: 17321950.

206. Wakahara T, Shiraki M, Murase K, et al. Nutritional screening with Subjective Global Assessment predicts hospital stay in patients with digestive diseases. Nutrition. 2007 Sep;23(9):634-9. http://dx.doi.org/10.1016/j.nut.2007.06.005. PMID: 17629455.
207. Kruizenga HM, de Jonge P, Seidell JC, et al. Are malnourished patients complex patients? Health status and care complexity of malnourished patients detected by the Short Nutritional Assessment Questionnaire (SNAQ). Eur J Intern Med. 2006 May;17(3):189-94. http://dx.doi.org/10.1016/j.ejim.2005.11.019. PMID: 16618452.

208. Kyle UG, Kossovsky MP, Karsegard VL, et al. Comparison of tools for nutritional assessment and screening at hospital admission: a population study. Clin Nutr. 2006 Jun;25(3):409-17. http://dx.doi.org/10.1016/j.clnu.2005.11.001. PMID: 16356595.

209. Stratton RJ, King CL, Stroud MA, et al. 'Malnutrition universal screening tool' predicts mortality and length of hospital stay in acutely ill elderly. Br J Nutr. 2006 Feb;95(2):325-30. http://dx.doi.org/10.1079/BJN20051622. PMID: 16469149.

210. Bouillanne O, Morineau G, Dupant C, et al. Geriatric nutritional risk index: a new index for evaluating at-risk elderly medical patients. Am J Clin Nutr. 2005;82(4):777-83. PMID: 16210706.

211. Kagansky N, Berner Y, Koren-Morag N, et al. Poor nutritional habits are predictors of poor outcome in very old hospitalized patients. Am J Clin Nutr. 2005;82(4):784-91. PMID: 16210707.

212. Norman K, Schüz T, Kemps M, et al. The Subjective Global Assessment reliably identifies malnutrition-related muscle dysfunction. Clin Nutr. 2005 Feb;24(1):14350. http://dx.doi.org/10.1016/j.clnu.2004.08.007. PMID: 15681112.

213. Pepersack T. Outcomes of continuous process improvement of nutritional care program among geriatric units. J Gerontol A Biol Sci Med Sci. 2005 Jun;60(6):787-92. http://dx.doi.org/10.1093/gerona/60.6.787. PMID: 15983184.

214. Thomas DR, Kamel H, Azharrudin M, et al. The relationship of functional status, nutritional assessment, and severity of illness to in-hospital mortality. J Nutr Health Aging. 2005;9(3):169-75. PMID: 15864397. 
215. Kyle UG, Pirlich M, Schuetz T, et al. Is nutritional depletion by nutritional risk index associated with increased length of hospital stay? A population-based study. JPEN J Parenter Enteral Nutr. 2004 Mar-

Apr;28(2):99-104. http://dx.doi.org/10.1177/01486071040280029 9. PMID: 15080604.

216. Pichard C, Kyle UG, Morabia A, et al. Nutritional assessment: lean body mass depletion at hospital admission is associated with an increased length of stay. Am J Clin Nutr. 2004 Apr;79(4):613-8. PMID: 15051605 .

217. Visvanathan R, Penhall R, Chapman I. Nutritional screening of older people in a subacute care facility in Australia and its relation to discharge outcomes. Age Ageing. 2004 May;33(3):260-5. http://dx.doi.org/10.1093/ageing/afh078. PMID: 15082431.

218. Bauer J, Capra S, Ferguson M. Use of the scored Patient-Generated Subjective Global Assessment (PG-SGA) as a nutrition assessment tool in patients with cancer. Eur J Clin Nutr. 2002;56(8):779-85. http://dx.doi.org/10.1038/sj.ejen.1601412. PMID: 12122555.
219. Persson MD, Brismar KE, Katzarski KS, et al. Nutritional status using Mini Nutritional Assessment and Subjective Global Assessment predict mortality in geriatric patients. J Am Geriatr Soc. 2002 Dec;50(12):1996-2002. http://dx.doi.org/10.1046/j.15325415.2002.50611.x. PMID: 12473011.

220. Kalantar-Zadeh K, Kopple JD, Block G, et al. A malnutrition-inflammation score is correlated with morbidity and mortality in maintenance hemodialysis patients. Am $\mathbf{J}$ Kidney Dis. 2001;38(6):1251-63. PMID: 11728958 .

221. Laws RA, Tapsell LC, Kelly J. Nutritional status and its relationship to quality of life in a sample of chronic hemodialysis patients. J Ren Nutr. 2000 Jul;10(3):139-47. PMID: 10921535.

222. Santoso JT, Canada T, Latson B, et al. Prognostic nutritional index in relation to hospital stay in women with gynecologic cancer. Obstet Gynecol. 2000 Jun;95(6):844-6. PMID: 10831978. 